Aprendizado de máquina com informação privilegiada: abordagens para agrupamento

hierárquico de textos 

SERVIÇO DE PÓS-GRADUAÇÃO DO ICMC-USP

Data de Depósito:

Assinatura:

\title{
Aprendizado de máquina com informação privilegiada: abordagens para agrupamento hierárquico de textos
}

\author{
Ricardo Marcondes Marcacini
}

Orientadora: Profa. Dra. Solange Oliveira Rezende

Coorientador: Prof. Dr. Eduardo Raul Hruschka

Tese apresentada ao Instituto de Ciências Matemáticas e de Computação - ICMC-USP, como parte dos requisitos para obtenção do título de Doutor em Ciências - Ciências de Computação e Matemática Computacional. EXEMPLAR DE DEFESA. 
Ficha catalográfica elaborada pela Biblioteca Prof. Achille Bassi e Seção Técnica de Informática, ICMC/USP, com os dados fornecidos pelo(a) autor(a)

Marcacini, Ricardo Marcondes
Aprendizado de Máquina com Informação
Privilegiada: Abordagens para Agrupamento
Hierárquico de Textos / Ricardo Marcondes
Marcacini; orientadora Solange Oliveira Rezende;
Co-orientador Eduardo Raul Hruschka. -- São Carlos,
2014.
178 p.
Tese (Doutorado - Programa de Pós-Graduação em
Ciências de Computação e Matemática Computacional) --
Instituto de Ciências Matemáticas e de Computação,
Universidade de São Paulo, 2014.
1. Aprendizado não Supervisionado de Máquina. 2.
Agrupamento de Textos. 3. Informação Privilegiada.
A. Aprendizado de Métricas. 5. Seleção de Modelos.
I. Rezende, Solange Oliveira, orient. II.
Hruschka, Eduardo Raul, co-orient. III. Título.




\section{Agradecimentos}

a Deus pelo sopro da vida e por Seu amor incondicional; à minha esposa, Eloní Midiani de Oliveira Marcacini, pelo companheirismo, paciência e apoio. Em especial, agradeço a ela por ter me dado o melhor presente, que é o(a) filho(a) que está gerando como fruto do nosso amor; aos meus pais, Marcondes Marcacini e Davínia Gomes Marcacini, por tudo que me ensinaram e por estarem ao meu lado em todos os momentos. Aos meus irmãos Renan e Renato que, mesmo com a distância dos últimos anos, sempre estiveram presentes na minha vida;

ao Zaqueu, Duzair, Eligilca, Nícolas e Victor Hugo, que carinhosamente me receberam em sua família e me apoiaram nos períodos difíceis;

à minha orientadora, Profa. Dra. Solange Oliveira Rezende, que tive o privilégio de conhecer e participar do seu trabalho. Com generosidade e sabedoria me orientou na vida acadêmica e na minha formação como ser

humano. Suas atitudes são exemplos que levarei sempre comigo. Serei eternamente agradecido por ter confiado e me dado esta oportunidade;

ao meu coorientador, Prof. Dr. Eduardo Raul Hruschka, que com grande competência e paciência forneceu valiosas contribuições que permitiram o desenvolvimento desta tese. Além disso, é um professor admirável que tive o prazer de ser aluno desde a graduação;

à todos os amigos e companheiros do Labic, pelos momentos alegres e ajuda indispensável durante a realização deste trabalho;

aos professores e funcionários do ICMC-USP e da UFMS. Em especial, aos docentes do curso de Sistemas de Informação da UFMS (CPTL);

ao Anandsing Dwarkasing, ou simplesmente Anand, pela generosidade e valiosa ajuda no inglês, mesmo com seu pouco tempo disponível;

à banca examinadora, que gentilmente aceitou o convite para essa defesa; e à FAPESP pelo apoio financeiro. 



\section{Resumo}

Métodos de agrupamento hierárquico de textos são muito úteis para analisar o conhecimento embutido em coleções textuais, organizando os documentos textuais em grupos e subgrupos para facilitar a exploração do conhecimento em diversos níveis de granularidade. Tais métodos pertencem à área de aprendizado não supervisionado de máquina, uma que vez obtêm modelos de agrupamento apenas pela observação de regularidades existentes na coleção textual, sem supervisão humana. Os métodos tradicionais de agrupamento assumem que a coleção textual é representada apenas pela "informação técnica", ou seja, palavras e frases extraídas diretamente dos textos. Por outro lado, em muitas tarefas de agrupamento existe conhecimento adicional e valioso a respeito dos dados, geralmente extraído por um processo avançado com apoio de usuários especialistas do domínio do problema. Devido ao alto custo para obtenção desses dados, esta informação adicional é definida como "privilegiada" e usualmente está disponivel para representar apenas um subconjunto dos documentos textuais. Recentemente, um novo paradigma de aprendizado de máquina denominado LUPI (Learning Using Privileged Information) foi proposto por Vapnik para incorporar informação privilegiada em métodos aprendizado supervisionado. Neste trabalho de doutorado, o paradigma LUPI foi estendido para aprendizado não supervisionado, em especial, para agrupamento hierárquico de textos. Foram propostas e avaliadas abordagens para lidar com diferentes desafios existentes em tarefas de agrupamento, envolvendo a extração e estruturação da informação privilegiada e seu uso para refinar ou corrigir modelos de agrupamento. As abordagens propostas se mostraram eficazes em (i) consenso de agrupamentos, permitindo combinar diferentes representações e soluções de agrupamento; (ii) aprendizado de métricas, em que medidas de proximidades mais robustas foram obtidas com base na informação privilegiada; e (iii) seleção de modelos, em que a informação privilegiada é explorada para identificar relevantes estruturas de agrupamento hierárquico. Todas as abordagens apresentadas foram investigadas em um cenário de agrupamento incremental, permitindo seu uso em aplicações práticas caracterizadas pela necessidade de eficiência computacional e alta frequência de publicação de novo conhecimento textual. 



\section{Abstract}

Hierarchical text clustering methods are very useful to analyze the implicit knowledge in textual collections, enabling the organization of textual documents into clusters and subclusters to facilitate the knowledge browsing at various levels of granularity. Such methods are classified as unsupervised machine learning, since the clustering models are obtained only by observing regularities of textual data without human supervision. Traditional clustering methods assume that the text collection is represented only by the "technical information", i.e., words and phrases extracted directly from the texts. On the other hand, in many text clustering tasks there is an additional and valuable knowledge about the problem domain, usually extracted by an advanced process with support of the domain experts. Due to the high cost of obtaining such expert knowledge, this additional information is defined as "privileged" and is usually available to represent only a subset of the textual documents. Recently, a new machine learning paradigm called LUPI (Learning Using Privileged Information) was proposed by Vapnik to incorporate privileged information into supervised learning methods. In this thesis, the LUPI paradigm was extended to unsupervised learning setting, in particular for hierarchical text clustering. We propose and evaluate approaches to deal with different challenges for clustering tasks, involving the extraction and structuring of privileged information and using this additional information to refine or correct clustering models. The proposed approaches were effective in (i) consensus clustering, allowing to combine different clustering solutions and textual representations; (ii) metric learning, in which more robust proximity measures are obtained from privileged information; and (iii) model selection, in which the privileged information is exploited to identify the relevant structures of hierarchical clustering. All the approaches presented in this thesis were investigated in an incremental clustering scenario, allowing its use in practical applications that require computational efficiency as well as deal with high frequency of publication of new textual knowledge. 



\section{Sumário}

\section{Sumário}

vii

Lista de Figuras

xi

Lista de Tabelas $\quad$ xv

1 Introdução 1

1.1 Definição do Problema e Desafios . . . . . . . . . . . . 1

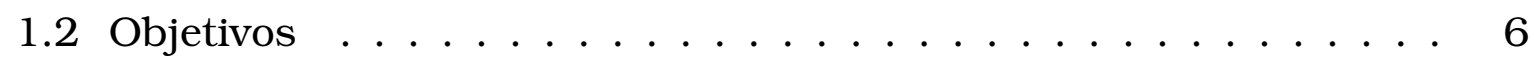

1.3 Principais Resultados . . . . . . . . . . . . . . . 8

1.4 Organização do Texto $\ldots \ldots \ldots \ldots \ldots \ldots$

2 Fundamentos 13

2.1 Considerações Iniciais . . . . . . . . . . . . . . . . . 13

2.2 Análise de Agrupamentos . . . . . . . . . . . . . . . . 14

2.2.1 Medidas de Proximidade . . . . . . . . . . . . . . . 15

2.2.2 Métodos de Agrupamento . . . . . . . . . . . . . . 16

2.2 .3 Avaliação de Agrupamentos . . . . . . . . . . . . . . . . 29

2.3 Aprendizado de Máquina com Informação Privilegiada . . . . . . 30

2.3.1 O paradigma LUPI (Learning using Privileged Information) . 33

2.3.2 Algoritmos e Aplicações baseados no paradigma LUPI . . 35

2.4 Considerações Finais . . . . . . . . . . . . . . . 41

3 Informação Privilegiada em Agrupamento Hierárquico de Textos 43

3.1 Considerações Iniciais . . . . . . . . . . . . . . . . 43 
3.2 Representação Estruturada para Dados Textuais . . . . . . . . . . 46

3.3 Seleção Interativa de Termos . . . . . . . . . . . . . . . . . . 53

3.4 A Ferramenta AL ${ }^{2}$ FIC para Extração de Informação Privilegiada 55

3.5 Abordagem Proposta: LIHC . . . . . . . . . . . . . . . . 58

3.5.1 Estratégia para Consenso de Agrupamentos . . . . . . . . 59

3.5.2 Aspectos Teóricos . . . . . . . . . . . . . . . 60

3.6 Análise Experimental . . . . . . . . . . . . . . . 61

3.6.1 Configuração dos Experimentos . . . . . . . . . . . 62

3.6.2 Simulando Interações com o Usuário . . . . . . . . . . . 63

3.6.3 Análise de Parâmetros . . . . . . . . . . . . . . . 63

3.6.4 Agrupamento Hierárquico Incremental . . . . . . . . . . 67

3.7 Considerações Finais . . . . . . . . . . . . . 71

4 Informação Privilegiada em Aprendizado de Métricas $\quad 73$

4.1 Considerações Iniciais . . . . . . . . . . . . . . . . 73

4.2 Trabalhos Relacionados . . . . . . . . . . . . . . . 75

4.2.1 A abordagem IPIML . . . . . . . . . . . . . . . . . 75

4.2.2 Aprendizado de Métricas em Agrupamento de Dados . . . . 78

4.2 .3 Análise Conceitual . . . . . . . . . . . . . . . . 80

4.3 Abordagens Propostas: IPIML-GLOBAL e IPIML-LOCAL . . . . . . . 81

4.3.1 Extração de Restrições Must-Link a partir de Informação Privilegiada ................. . . 82

4.3.2 Aprendizado de Métricas para Agrupamento Hierárquico de Documentos . . . . . . . . . . . . . . . 83

4.4 Análise Experimental . . . . . . . . . . . . . . 87

4.5 Considerações Finais . . . . . . . . . . . . . . . 93

5 Informação Privilegiada para Seleção de Modelos 95

5.1 Considerações Iniciais . . . . . . . . . . . . . . . . . 95

5.2 Trabalhos Relacionados . . . . . . . . . . . . . . 97

5.2.1 Critério Relativo . . . . . . . . . . . . . . . . . . . 98

5.2 .2 Critério Externo . . . . . . . . . . . . . . . 99

5.2.3 Estabilidade do Modelo . . . . . . . . . . . . . . 100

5.2.4 A Abordagem aRi-MAX . . . . . . . . . . . . . . . 102

5.3 Abordagens propostas: cdi-MAX e edi-MAX . . . . . . . . . . . 103

5.3.1 Cophenetic Difference Index (cdi) . . . . . . . . . . . . . . . . 104

5.3 .2 Edge Distance Index (edi) . . . . . . . . . . . . . . . . . . 106

5.4 Aspectos Teóricos . . . . . . . . . . . . . . 107 
5.5 Análise Experimental . . . . . . . . . . . . . . . . . . 108

5.6 Considerações Finais $\ldots \ldots \ldots$. . . . . . . . . . . . . 114

6 Aplicações $\quad 117$

6.1 Considerações Iniciais . . . . . . . . . . . . . . . . . 117

6.2 Análise Exploratória de Mídias Sociais . . . . . . . . . . . . . 117

6.2.1 Extração da Informação Privilegiada . . . . . . . . . . . . 119

6.2.2 Seleção de Conteúdo para Discurso do Sujeito Coletivo . 119

6.2 .3 Discussão dos Resultados . . . . . . . . . . . . . . . . . 122

6.3 Sistemas de Recomendação Sensíveis ao Contexto . . . . . . . . 122

6.3.1 Entidades Nomeadas como Informação Privilegiada . . . . . 124

6.3.2 Recomendação baseada em Filtragem Colaborativa . . . . . 125

6.3.3 Discussão dos Resultados . . . . . . . . . . . . . . . . 127

6.4 Considerações Finais . . . . . . . . . . . . . . . . . . . . . . 128

7 Conclusões 131

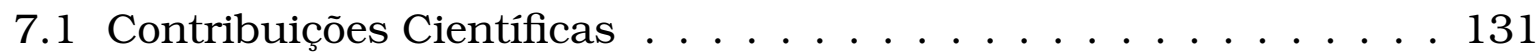

7.2 Publicações Relacionadas . . . . . . . . . . . . . . . . . . 136

7.3 Limitações e Trabalhos Futuros . . . . . . . . . . . . . . . . 138

$\begin{array}{ll}\text { Referências Bibliográficas } & 141\end{array}$ 



\section{Lista de Figuras}

2.1 Exemplo de um dendrograma (adaptado de Xu e Wunsch (2008)) . . 19

2.2 Visão geral do número de publicações que discutem ou investigam a abordagem LUPI (Learning Using Privileged Information) de 2009 a maio de 2014. . . . . . . . . . . . . . . . 35

3.1 Etapas do processo de extração de informação privilegiada por meio da ferramenta AL ${ }^{2}$ FIC: (A) agrupamento baseado em itemsets, (B) seleção interativa de termos, e (C) extração de uma nova representação estruturada baseada nos itemsets selecionados. . . . . . . . . . 56

3.2 Visão geral da interface da ferramenta $\mathrm{AL}^{2} \mathrm{FIC}$ utilizada para extrair informação privilegiada em coleções textuais. . . . . . . . . . . 58

3.3 Valores de $F_{S C O R E}$ para cada coleção textual referentes à análise dos parâmetros de Fator de Contribuição $(\alpha)$ e número de itemsets sele-

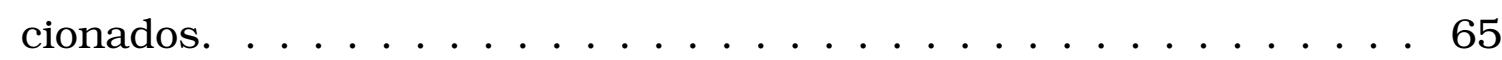

3.4 Valores $F_{S C O R E}$ para consenso de agrupamentos considerando 100 itemsets na representação textual de informação privilegiada. . . . . 66

3.5 Valores $F_{S C O R E}$ considerando diferentes níveis de consulta aos usuários para cada coleção textual. . . . . . . . . . . . . 66

3.6 Comparação do índice $F_{S C O R E}$ entre as abordagens $L I H C$ e CC durante o processo de agrupamento. A etapa $S_{0}$ representa a acurácia do modelo inicial e $S_{1} 0$ a acurácia da solução final de agrupamento. . 69 
3.7 Nivel de melhoria da solução de agrupamento obtida pela abordagem LIHC (LUPI-Based Incremental Hierarchical Clustering) em relação ao CC (Consensus Clustering - sem informação Privilegiada). Para facilitar a visualização, o valor do nível é calculado por meio da raiz quadrada da porcentagem de melhora obtida pelo LIHC. . . . . . . . 70

4.1 Ilustração do processo de extração de restrições do tipo Must-Link conforme a estrutura de agrupamento. No grafo, dois pontos estão conectados por uma aresta se a distância entre eles é considerada

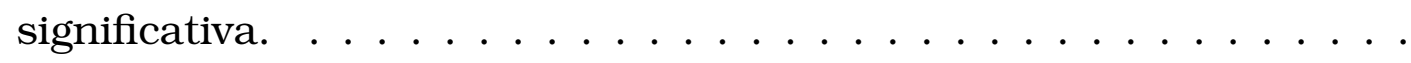

4.2 Análise comparativa da acurácia de agrupamento, no gráfico à esquerda, entre IPIML-GLOBAL e IPIML-LOCAL. No gráfico à direita são apresentados (i) a porcentagem de restrições incorretas e (ii) o número de restrições de acordo com o nível da hierarquia. . . . . . . . .

4.3 Análise comparativa da acurácia de agrupamento, no gráfico à esquerda, entre IPIML-GLOBAL e IPIML-LOCAL. No gráfico à direita são apresentados (i) a porcentagem de restrições incorretas e (ii) o número de restrições de acordo com o nível da hierarquia. . . . . . . . . 90

4.4 Porcentagem de melhora da solução de agrupamento com uso das abordagens IPIML-GLOBAL e IPIML-LOCAL em relação ao Baseline. .

4.5 Diferença crítica $(C D)$ sobre o ranking das abordagens exploradas de acordo com a acurácia $\left(F_{S C O R E}\right)$ da solução final de agrupamento.

5.1 Exemplo de matrizes de descritores $C D$ e $E D$ extraídas de um dendrograma. O dendrograma foi gerado com uso do algoritmo de agrupamento hierárquico Single-Linkage a partir da referida matriz de

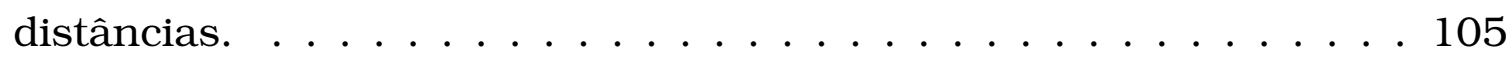

5.2 Dendrograma com identificação de nível $(N L)$ para cada subgrupo. . 106

5.3 Reconstrução do dendrograma a partir da matriz de descritores. (a) Dendrograma reconstruído a partir da matriz de descritores $C D$ é igual ao dendrograma original. (b) Dendrograma reconstruído a partir da matriz de descritores $E D$ recupera a topologia do dendrograma original. . . . . . . . . . . . . . . . 108

5.4 Comparação do índice $F_{S C O R E}$ das abordagens aRi-MAX, cdi-MAX e edi-MAX durante o processo de agrupamento. A etapa $S_{0}$ representa a acurácia do modelo inicial e $S_{1} 0$ a acurácia da solução final de agrupamento. 
5.5 Porcentagem de ganho/perda das abordagens aRi-MAX, cdi-MAX e edi-MAX em relação ao Baseline. . . . . . . . . . . . . . . . . . 113

5.6 Diferença crítica $(C D)$ sobre o ranking das abordagens exploradas de acordo com a acurácia $\left(F_{S C O R E}\right)$ da solução final de agrupamento. 113

6.1 Interface para seleção de itemsets e exploração da rede de coocorrência de termos da ferramenta TorchSR. . . . . . . . . . . . . . . 120

6.2 Interface para apoiar a seleção de conteúdo textual a ser utilizado para análise do Discurso do Sujeito Coletivo (DSV) . . . . . . . . . . 121

6.3 Comparação da acurácia do sistema recomendação entre IBCF (não utiliza contexto), Filter PoF ( $\alpha=0)$ que utiliza contexto sem informação privilegiada, e Filter $\operatorname{PoF}(\alpha=0.5)$ que utiliza contexto baseado na informação privilegiada. . . . . . . . . . . . . . . . 128 



\section{Lista de Tabelas}

2.1 Resumo dos principais trabalhos discutidos que abordagem o paradigma LUPI em problemas de aprendizado de máquina. . . . . . . . . 41

3.1 Modelo espaço-vetorial para representação de dados textuais . . . 46

3.2 Detalhes das Coleções Textuais usadas na Análise de Parâmetros. . 64

3.3 Detalhes das coleções textuais utilizadas na avaliação experimental 68

5.1 Tabela com matrizes VED (Vitória, Empate e Derrota) entre as abordagens em cada coleção textual. Em cada matriz VED, compara-se a abordagem da linha contra a abordagem da coluna. Os valores $v, e, d$, representam, respectivamente, as porcentagens de vitória, empate e derrota. . . . . . . . . . . . . . . . . 111

6.1 Lista de 20 termos mais frequentes das representações de informação técnica e informação privilegiada. 



\section{CAPÍTULO}

\section{1}

\section{Introdução}

\subsection{Definição do Problema e Desafios}

Métodos de agrupamento hierárquico de textos são muito populares para a organização não supervisionada do conhecimento implícito em coleções textuais (Zhao et al., 2005; Aggarwal e Zhai, 2012b; Anastasiu et al., 2013). Nesses métodos, os exemplos (documentos textuais) são organizados em um modelo de agrupamento, de forma que exemplos pertencentes a um mesmo grupo sejam similares entre si e, ao mesmo tempo, dissimilares aos exemplos de outros grupos (Jain et al., 1999; Xu e Wunsch, 2008; Rokach, 2010; Aggarwal e Zhai, 2012b). Ainda, a organização é disposta em uma estrutura hierárquica, na qual os grupos próximos à raiz da hierarquia representam conhecimento mais geral, enquanto os subgrupos representam detalhamento e conhecimento mais específico (Zhao et al., 2005). Assim, é possível explorar a organização dos documentos em diversos níveis de abstração, bem como analisar um grande volume de dados (Aggarwal e Zhai, 2012b).

Os métodos de agrupamento também são conhecidos como algoritmos de aprendizado não supervisionado ou de análise exploratória dos dados, pois o modelo de agrupamento é obtido pela observação de padrões nos dados, geralmente baseada em uma medida que expressa uma noção de proximidade entre os exemplos (Xu e Wunsch, 2008). Devido à capacidade de aprender modelos de agrupamento com pouca (ou nenhuma) supervisão humana, métodos de agrupamento hierárquico de textos têm desempenhado um papel relevante em diversos estudos na área de aprendizado não supervisionado de máquina (Hastie et al., 2009) e, também, em aplicações relacionadas à mineração de 
textos e recuperação de informação (Manning et al., 2008; Aggarwal e Zhai, 2012a).

Apesar dos recentes avanços no desenvolvimento de métodos para agrupamento hierárquico de textos, ainda existem desafios de pesquisa em aberto que limitam o desempenho desses métodos. A seguir, são descritos os desafios de pesquisa que estão mais relacionados ao contexto deste trabalho de doutorado.

Múltiplas Representações para Dados Textuais: Dados textuais são inerentemente não estruturados, necessitando de uma etapa de pré-processamento para extrair as características dos textos e representá-los em um formato estruturado (Jiang, 2012). Existem diversas técnicas para este fim, desde as mais tradicionais, como a geração de uma bag-of-words (Salton e Buckley, 1988; Salton et al., 1996), até técnicas mais avançadas, como extração de conceitos específicos de domínio e entidades nomeadas (Bechet, 2011; Jiang, 2012). Cada uma dessas técnicas obtém uma representação textual diferente do mesmo conjunto de exemplos e, em geral, cada representação permite revelar diferentes estruturas de agrupamento. Um desafio de pesquisa é investigar métodos de agrupamento que exploram as diferentes representações durante a formação dos grupos para, então, fornecer um modelo de agrupamento final que seja superior aos modelos obtidos com cada representação de forma individual (Bickel e Scheffer, 2004; Long et al., 2008; Günnemann et al., 2012; Joshi et al., 2014). Além disso, há outros desafios específicos para este cenário, por exemplo, definir o nível de importância de cada representação textual durante o aprendizado do modelo de agrupamento.

Aprendizado de Métricas: A eficácia de vários métodos de agrupamento depende da escolha de uma medida proximidade "adequada" (Xing et al., 2002; Fouad e Tino, 2013). No entanto, esta escolha é uma decisão complexa, dada a dificuldade em definir a noção de "adequado", principalmente no caso de dados textuais devido à alta dimensionalidade (Lebanon, 2006). Um desafio de pesquisa é a investigação de métodos para aprendizado de métricas, que visam computar uma medida de proximidade de acordo com as características específicas de um determinado conjunto de exemplos (Fouad et al., 2013). Para tal, podem ser utilizadas algumas informações adicionais sobre os dados, por exemplo, restrições que identificam alguns pares de exemplos que devem (ou não) ser aloca- 
dos no mesmo grupo (Bilenko et al., 2004; Covões et al., 2013), também chamadas de restrições Must-Link e Cannot-Link (Xiang et al., 2008). No entanto, nem sempre essas restrições estão disponiveis, bem como são difíceis de serem fornecidas pelos usuários devido à natureza não supervisionada do problema (Lajugie et al., 2014).

Seleção de Modelos de Agrupamento: A existência de diversos algoritmos para agrupamento de textos, e de diferentes parâmetros de um mesmo mesmo algoritmo, leva ao seguinte questionamento: dentre vários modelos de agrupamento, como selecionar o modelo mais apropriado? A seleção de modelos de agrupamento é um relevante desafio de pesquisa, uma vez que algoritmos de agrupamento sempre encontram grupos nos dados, independentemente de serem reais ou não (Vaithyanathan e Dom, 1999; Lange et al., 2002). Apesar da existência de critérios estatísticos que verificam o quão bem um modelo de agrupamento se ajusta ao conjunto de dados, o uso desses critérios assume que uma determinada medida de proximidade é adequada, ou que os dados possuem uma determinada distribuição. Tais premissas são de difícil comprovação em cenários práticos (Vendramin et al., 2010). Ainda, a maioria desses critérios não exploram as relações de grupos e subgrupos provenientes do agrupamento hierárquico, ou seja, ignoram informações da estrutura hierárquica que são importantes para modelar os dados em diversos níveis de granularidade.

Agrupamento Incremental: Com o surgimento e popularização das plataformas on-line para publicação de conteúdo textual, o volume de dados textuais armazenado nos repositórios textuais cresce diariamente. Os métodos tradicionais de agrupamento não são eficazes para este cenário, pois consideram que o conjunto de exemplos é estático (Sahoo et al., 2006; Bouchachia, 2008; Aggarwal e Reddy, 2013). Desse modo, após inserção de novo conteúdo é necessário repetir todo o processo de agrupamento. Esta solução é computacionalmente custosa e muitas vezes inviável na prática (Garcia e Porrata, 2010). Nesse sentido, um desafio de pesquisa é a investigação de métodos de agrupamento incremental, de forma a permitir a inserção de novos exemplos a um modelo de agrupamento previamente existente, sem a necessidade de reprocessamento redundante.

Um recente paradigma de aprendizado denominado LUPI (Learning Using 
Privileged Information) (Vapnik e Vashist, 2009), proposto originalmente para aprendizado supervisionado, apresenta uma estratégia interessante para lidar com esses tipos de desafios de forma unificada. No paradigma LUPI, as múltiplas representações dos dados são organizadas em dois tipos: informação técnica e informação privilegiada (Pechyony e Vapnik, 2010). A informação técnica é uma representação dos dados obtida a partir de um processo técnico, de baixo custo (em termos de recursos humanos e computacionais) e altamente disponível para descrever os exemplos. Devido a esta alta disponibilidade, a representação de informação técnica geralmente é o alvo para um processo aprendizado. Já a informação privilegiada é uma representação gerada a partir de um processo avançado, de alto custo, dependente de domínio, e que contém uma informação adicional, potencialmente valiosa, a respeito dos exemplos. Devido ao alto custo para obtenção desses dados, a informação privilegiada está disponível para representar apenas um pequeno subconjunto dos exemplos.

O processo de aprendizado de um modelo, utilizando o paradigma LUPI, possui duas fases (Vapnik e Vashist, 2009; Pechyony e Vapnik, 2010; Lapin et al., 2014). Na primeira fase, o subconjunto dos exemplos representados em ambas as representações - informação técnica e informação privilegiada - é utilizado para o aprendizado de um modelo. Na segunda fase, o restante dos exemplos, aqueles representados apenas pela informação técnica, é apresentado e incorporado ao modelo. Dessa forma, a representação de informação privilegiada é empregada apenas na primeira fase, geralmente para corrigir ou refinar o processo de aprendizado do modelo. Embora seja possível realizar o aprendizado usando apenas a informação técnica, o aprendizado pode ser melhorado na presença de informação adicional e valiosa a respeito dos exemplos. Vapnik e Vashist (2009) afirmam que em praticamente todos os domínios há algum tipo de informação privilegiada que, em geral, não é considerada em paradigmas tradicionais de aprendizado.

O estudo de algoritmos e aplicações para aprendizado não supervisionado com base no paradigma LUPI é um tópico de pesquisa em aberto, e que é abordado neste trabalho de doutorado. Em particular, o paradigma LUPI é investigado como uma alternativa promissora para agrupamento hierárquico de textos, pois permite lidar naturalmente com os desafios apresentados, como discutidos a seguir:

- As múltiplas representações dos dados textuais podem ser facilmente divididas em informação técnica e informação privilegiada. A estrutura 
bag-of-words, por exemplo, é um exemplo de informação técnica, uma vez que é facilmente extraída dos textos, além de ser altamente disponível. Assim, a descrição bag-of-words geralmente é o alvo principal em processos de agrupamento. Por outro lado, é possível extrair características mais avançadas dos textos, como conceitos específicos de domínio e entidades nomeadas. Essas características permitem obter representações de mais alto nível dos textos. Entretanto, a obtenção de tais características geralmente incorre em alto custo computacional e requer informação adicional específica do domínio, muitas vezes dependendo de consulta à usuários especialistas. Devido a essas limitações, em geral apenas uma pequena parcela dos exemplos serão representados nessas descrições mais avançadas, ou seja, esses exemplos representam a informação privilegiada do problema;

- O processo de aprendizado do paradigma LUPI é baseado em duas fases. Na primeira fase, um modelo inicial é aprendido com base no conjunto de exemplos representado tanto pela informação técnica quanto pela informação privilegiada. É neste ponto que os desafios relacionados à combinação das soluções de agrupamento provenientes de diferentes representações podem ser explorados. Nesse caso, incorporar informação privilegiada para aprendizado de métricas e também para seleção de modelos é uma estratégia promissora para refinar (ou corrigir) o aprendizado do modelo inicial. Já na segunda fase, os exemplos representados apenas pela informação técnica são incorporados ao modelo de forma incremental. Este cenário se torna importante em relação ao desafio de agrupamento incremental de textos.

O paradigma LUPI foi proposto originalmente para estender o algoritmo de aprendizado supervisionado SVM (Support Vector Machine) (Cortes e Vapnik, 1995) para o $S V M+$ (Vapnik e Vashist, 2009), que incorpora informação privilegiada visando melhorar o desempenho de modelos preditivos usados em problemas de classificação e regressão. Apesar da relevância do tema, ainda há poucos estudos na literatura que exploram o paradigma LUPI em tarefas de agrupamento (Feyereisl e Aickelin, 2012). 


\subsection{Objetivos}

Motivado pelas lacunas na área discutidas na Seção 1.1 e pela necessidade de métodos de agrupamento que sejam úteis às necessidades atuais, o propósito geral deste trabalho de doutorado é estender o paradigma LUPI (Learning Using Privileged Information) para agrupamento hierárquico de textos. Como resultado, são apresentadas novas abordagens para explorar as várias representações dos textos em métodos de aprendizado não supervisionado, contribuindo diretamente com os desafios de pesquisa descritos anteriormente.

O propósito geral desse projeto pode ser organizado em quatro objetivos que tratam de pontos de pesquisa ainda em aberto:

1. O paradigma LUPI foi proposto originalmente para aprendizado supervisionado, com foco em tarefas de classificação. Embora alguns estudos iniciais já tenham sido reportados por Feyereisl e Aickelin (2012) considerando tarefas de aprendizado não supervisionado, ainda há diversos aspectos desse paradigma a serem discutidos, principalmente envolvendo dados textuais. Investigar a importância da representação de informação privilegiada em tarefas de agrupamento de textos é um dos objetivos deste trabalho de doutorado. Nesse sentido, também é relevante definir quando uma representação textual pode ser considerada informação privilegiada e como extrair tal informação de um conjunto de textos. Por fim, discutir a contribuição prática do paradigma LUPI para agrupamento hierárquico de textos também faz parte deste objetivo, por meio da análise das possíveis aplicações que podem ser beneficiadas com essas abordagens. Baseado nesse objetivo, algumas perguntas guiam o desenvolvimento do trabalho:

- Gual a importância da informação privilegiada para aprendizado não supervisionado de máquina?

- Como extrair informação privilegiada a partir de textos?

- Guais tipos de aplicações podem ser beneficiadas?

2. As múltiplas representações dos dados textuais, organizadas em informação técnica e informação privilegiada, permitem obter diferentes soluções de agrupamento. Nesse sentido, um objetivo deste trabalho é propor e avaliar uma abordagem para combinar os diversos modelos de agrupamento provenientes da informação técnica e informação privilegiada 
em um modelo consensual, potencialmente mais robusto que os modelos individuais. Em relação a este objetivo, também é relevante investigar a importância (ou peso) dessas diferentes representações durante o aprendizado deste modelo consensual, bem como analisar o efeito desses modelos na etapa de agrupamento incremental. Assim, as seguintes perguntas norteiam este trabalho:

- Como combinar, em um único modelo de agrupamento, os diferentes modelos provenientes das representações de informação técnica e informação privilegiada?

- Gual o ganho que a incorporação de informação privilegiada proporciona para a etapa de agrupamento incremental?

- Gual a importância de cada representação para o modelo final de agrupamento?

3. O aprendizado de métricas considerando características específicas do conjunto de exemplos é um dos desafios aqui investigados. Um objetivo deste trabalho de doutorado é desenvolver uma abordagem que explore a representação de informação privilegiada para aprender uma nova medida de proximidade. Esta nova medida é empregada para definir de forma mais eficaz a relação de proximidade entre exemplos na representação de informação técnica. Desse modo, a informação privilegiada é incorporada naturalmente em diversos algoritmos de agrupamento de textos baseado em distâncias. Devido à natureza hierárquica do problema aqui investigado, também faz parte deste objetivo investigar o aprendizado de uma métrica global a ser utilizada durante todo o agrupamento ou, alternativamente, métricas locais considerando os diversos grupos e subgrupos do modelo de agrupamento hierárquico. Em resumo, as seguintes perguntas guiam o trabalho:

- Como utilizar a informação privilegiada para aprender novas métricas?

- Gual o ganho para o agrupamento de textos no espaço de informação técnica ao utilizar as métricas aprendidas no espaço de informação privilegiada?

- Quais as vantagens e desvantagens entre empregar uma medida de proximidade global e várias medidas de proximidade locais? 
4. Para lidar com o desafio de selecionar um modelo de agrupamento adequado, dentre um conjunto de modelos, um objetivo deste trabalho é desenvolver abordagens que utilizam a informação privilegiada para guiar a etapa de seleção de modelos. Nesse caso, modelos de agrupamento baseado em informação privilegiada são utilizados como referência na seleção de modelos de informação técnica. Também faz parte deste objetivo investigar abordagens mais apropriadas para agrupamento hierárquico, ou seja, que consideram informações sobre a estrutura topológica da hierarquia de grupos durante a seleção de modelos. No contexto deste objetivo, os seguintes questionamentos guiam o desenvolvimento do trabalho:

- Por que a informação privilegiada é interessante para seleção de modelos de agrupamento obtidos a partir da informação técnica?

- Como selecionar modelos de agrupamento hierárquico com apoio de informação privilegiada?

- As informações sobre a estrutura topológica da hierarquia são importantes para a seleção de modelos?

\subsection{Principais Resultados}

Com o intuito de estender o paradigma LUPI (Learning Using Privileged Information) para cenários não supervisionados, em particular, para agrupamento hierárquico de textos, foram realizadas as seguintes contribuições científicas:

- Uma abordagem para extrair informação privilegiada a partir de textos foi proposta com base em aprendizado ativo para seleção interativa de termos (Marcacini et al., 2012b). Com base nesta abordagem, foi desenvolvida uma ferramenta computacional que permite interação com usuários especialistas para extração de um conjunto de termos específicos de domínio. Uma análise experimental realizada em coleções compostas por artigos científicos da área da computação indica que o uso da representação de informação privilegiada constituída a partir desses conjuntos de termos aumenta significativamente a acurácia dos modelos de agrupamento;

- Foram investigadas abordagens baseadas em consenso de agrupamentos para combinar diferentes soluções de agrupamento, bem como seu efeito 
em agrupamento incremental (Marcacini et al., 2012c). Uma nova abordagem foi proposta, denominada LIHC (LUPI-Based Incremental Hierarchical Clustering) (Marcacini e Rezende, 2013), que atua principalmente no consenso dos agrupamentos provenientes da informação técnica e informação privilegiada. Avaliações experimentais em diversas coleções textuais de benchmark forneceram evidências de que a abordagem LIHC apresenta soluções superiores aos métodos de consenso de agrupamentos que não consideram a informação privilegiada do problema.

- A importância de cada representação, informação técnica e informação privilegiada, durante a combinação de diferentes modelos de agrupamento também foi investigada. Como contribuição, foi proposto um "Fator de Contribuição" das representações para consenso de agrupamentos (Correa et al., 2014). Os resultados experimentais indicam que a escolha de um valor equilibrado para o Fator de Contribuição, ou seja, que define importâncias similares para as duas representações, leva a melhores resultados em relação à acurácia do modelo de agrupamento.

- Foram propostas e avaliadas duas abordagens que incorporam informação privilegiada em aprendizado de métricas: IPIML-GLOBAL (Incorporating Privileged Information by Global Metric Learning) e IPIML-LOCAL (Incorporating Privileged Information by Local Metric Learning) (Marcacini et al., 2014). As avaliações experimentais realizadas em diversas coleções textuais de benchmark indicam que as medidas de proximidade obtidas com base na informação privilegiada melhoram significativamente a acurácia do modelo de agrupamento, comparado com o uso de uma medida tradicional de similaridade em textos. Em particular, o aprendizado de medidas locais, considerando diferentes níveis do agrupamento hierárquico, é mais eficaz do que o uso de uma medida global.

- O uso de informação privilegiada se mostrou uma alternativa promissora para o desafio de seleção de modelos. Neste trabalho de doutorado foram propostas duas abordagens: cdi-MAX (Maximum Cophenetic Difference Index) e edi-MAX (Maximum Edge Distance Index). Estas abordagens foram comparadas experimentalmente com a abordagem aRi-MAX (Maximum Adjusted Rand Index) proposta por Feyereisl e Aickelin (2012). Ao contrário da aRi-MAX, que é baseada apenas em partições dos dados, as abordagens cdi-MAX e edi-MAX utilizam dados sobre a estrutura hierárquica dos modelos extraídos da informação privilegiada. Os resultados 
experimentais fornecem evidências de que a tarefa de seleção de modelos com uso de informação privilegiada é mais efetiva quando são considerados a topologia da árvore e valores de fusão de grupos e subgrupos.

- As abordagens propostas neste trabalho de doutorado foram empregadas em duas aplicações, com o intuito de analisar o ganho em cenários práticos. A primeira aplicação é relacionada com análise exploratória de mídias sociais. Neste caso, a abordagem LIHC foi empregada para apoiar um grupo de pesquisadores da PUC-Rio na seleção de conteúdo relevante na temática "discussões em comunidades virtuais na Internet sobre o uso de crack, cocaína e maconha", utilizado para pesquisa na área de Ciências Sociais. Foi desenvolvida uma ferramenta, denominada TorchSR, que apoia todo o processo de análise exploratória, como a seleção de termos representativos de domínio, agrupamento hierárquico e seleção de conteúdo relevante (Carvalho et al., 2012b, 2014). A segunda aplicação envolve o uso da informação privilegiada para apoiar sistemas de recomendação sensiveis ao contexto. Nesse caso, modelos de agrupamento obtidos com uso de informação privilegiada são utilizados para refinar as sugestões de itens, apresentando melhores resultados do que modelos tradicionais de recomendação (Sundermann et al., 2014; Domingues et al., 2014a).

Além das contribuições relacionadas ao foco principal da tese, no trabalho de Sinoara et al. (2014) foi investigada a eficácia de representações textuais baseada na extração de entidades nomeadas como informação privilegiada. Outras estratégias para incorporar modelos de agrupamento em sistemas de recomendação sensiveis ao contexto, com base nas abordagens propostas nesta tese foram investigadas em Manzato et al. (2014) e Domingues et al. (2014b), apresentando resultados promissores. Por fim, também é importante ressaltar as contribuições relacionadas à como selecionar um subconjunto representativo de documentos textuais (Bonin et al., 2014) - tarefa útil para apoiar o aprendizado do modelo inicial. Outra contribuição está relacionada ao pré-processamento de textos em cenários incrementais, em que foram avaliadas diferentes técnicas de extração de palavras-chave para algoritmos de agrupamento incremental (Rossi et al., 2013b, 2014). 


\subsection{Organização do Texto}

O restante deste trabalho está organizado da seguinte maneira:

Cap. 2 - Fundamentos. Neste capítulo são apresentados os conceitos básicos sobre análise de agrupamentos, com foco em dados textuais. Em seguida, também são apresentados os fundamentos, motivações e uma revisão da literatura com os principais trabalhos sobre aprendizado de máquina com informação privilegiada.

Cap. 3 - Informação Privilegiada em Agrupamento Hierárquico de Textos. Os desafios de pesquisa relacionados ao primeiro objetivo são tratados neste capítulo. Em particular, é proposta e avaliada a abordagem para extração de informação privilegiada em textos, bem como é apresentada a ferramenta desenvolvida para este fim. A abordagem LIHC proposta para combinar diferentes modelos de agrupamento, provenientes das representações de informação privilegiada e técnica, é discutida e avaliada, bem como aspectos relacionados ao agrupamento incremental.

Cap. 4 - Informação Privilegiada em Aprendizado de Métricas. Os desafios de pesquisa sobre aprendizado de métricas em tarefas de agrupamento são explorados neste capítulo. Em particular, são apresentadas e avaliadas abordagens que incorporam aprendizado de métricas com apoio de informação privilegiada.

Cap. 5 - Informação Privilegiada para Seleção de Modelos. As abordagens propostas para explorar a informação privilegiada na tarefa de seleção de modelos são discutidas e avaliadas neste capítulo.

Cap. 6 - Aplicações. Neste capítulo são discutidas a significância prática do paradigma LUPI em tarefas de agrupamento. Em especial, são apresentadas aplicações em que as abordagens propostas nesta tese são empregadas para apoiar análise exploratória de textos e melhorar a eficácia de sistemas de recomendação.

Cap. 7 - Conclusões. As considerações finais desta tese são apresentadas. Os objetivos e respectivas questões de pesquisa propostas na introdução da tese são retomados e discutidos. Por fim, são descritas as limitações deste trabalho e as direções para trabalhos futuros. 



\section{CAPÍTULO}

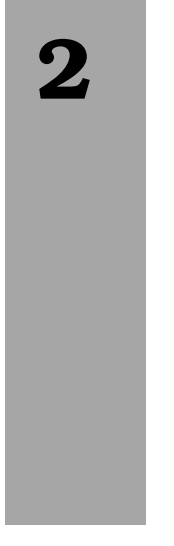

\section{Fundamentos}

\subsection{Considerações Iniciais}

Aprendizado de Máquina é uma área que envolve o desenvolvimento de métodos computacionais que são capazes de "aprender" um determinado comportamento a partir de exemplos, observações e experiências acumuladas (Mitchell, 1997; Duda et al., 2000; Bishop, 2007; Flach, 2012). Em geral, tais métodos podem ser organizados em três tipos: aprendizado supervisionado, aprendizado semissupervisionado e aprendizado não supervisionado. Em aprendizado supervisionado é fornecido um conjunto de treinamento formado pelos exemplos e seus respectivos rótulos. Nesse caso, o objetivo do aprendizado é utilizar o conjunto de treinamento para aprender uma função de mapeamento entre os exemplos e rótulos (Flach, 2012). É esperado que tal função seja capaz de mapear os exemplos não vistos, chamado de conjunto de teste, aos prováveis rótulos. Guando os rótulos são definidos como valores discretos sem relação de ordem, então o método de aprendizado é chamado de classificação. Quando os valores são contínuos ou possuem alguma relação de ordem, então o método de aprendizado é chamado de regressão. O mesmo tipo de informação está disponível em aprendizado semissupervisionado, porém a quantidade de exemplos rotulados é muito menor, necessitando de métodos específicos para lidar com este cenário (Chapelle et al., 2010). Outros métodos de aprendizado semissupervisionado contam com um conjunto de restrições impostas durante a tarefa de aprendizado, em adição ou substituição ao conjunto de exemplos rotulados (Basu et al., 2008).

Já em aprendizado não supervisionado não há exemplos rotulados e o ob- 
jetivo do aprendizado consiste em identificar padrões ou revelar estruturas intrínsecas embutidas nos dados (Flach, 2012; Mohri et al., 2012). Diversos métodos para aprendizado não supervisionado foram propostos, mas os mais populares são métodos de aprendizado para Associação (Tan et al., 2005; Flach, 2012) e Análise de Agrupamentos (Jain et al., 1999; Everitt et al., 2001; Xu e Wunsch, 2008; Aggarwal e Reddy, 2013). Os métodos para Análise de Agrupamentos são de interesse deste trabalho de doutorado.

Neste capítulo são apresentados os conceitos básicos relacionados a este trabalho, divididos em duas seções principais. Na Seção 2.2, uma visão geral sobre análise de agrupamentos no contexto de dados textuais é apresentada, incluindo medidas de proximidade, estratégias de agrupamento e avaliação de modelos de agrupamento. Já na Seção 2.3, os conceitos básicos e principais algoritmos e aplicações relacionados ao paradigma LUPI (Learning Using Privileged Information) são discutidos. Tais trabalhos foram analisados, durante este trabalho de doutorado, visando identificar motivações e lacunas na área, comentadas brevemente durante este capítulo. Uma revisão mais profunda de algumas abordagens de interesse direto deste trabalho é realizada nos respectivos capítulos da tese, de acordo com o tema abordado. Assim, o leitor familiarizado com a literatura clássica de agrupamento de dados e com aprendizado baseado em informação privilegiada pode saltar esse capítulo sem comprometer o entendimento do material abordado nos capítulos seguintes.

\subsection{Análise de Agrupamentos}

Em análise de agrupamentos, o objetivo é organizar um conjunto de exemplos em grupos baseando-se em uma medida de proximidade, na qual exemplos de um mesmo grupo são altamente similares entre si, mas dissimilares em relação aos exemplos de outros grupos (Xu e Wunsch, 2008). Em outras palavras, o agrupamento é baseado no princípio de maximizar a similaridade interna dos grupos (intragrupo) e minimizar a similaridade entre os grupos (intergrupos) (Aggarwal e Reddy, 2013). A análise de agrupamentos também é conhecida como aprendizado por observação ou análise exploratória dos dados, pois a organização dos exemplos em grupos é realizada apenas pela observação de regularidades nos dados, sem (ou com pouca) supervisão humana. Assim, ao contrário de métodos supervisionados, como na indução de classificadores, em processos de agrupamento não há classes ou rótulos predefinidos para treinamento de um modelo, ou seja, o aprendizado é reali- 
zado de forma não supervisionada (Jain et al., 1999; Rokach, 2010; Aggarwal e Zhai, 2012b).

O processo de agrupamento depende de dois fatores principais: (1) uma medida de proximidade e (2) uma estratégia de agrupamento. As medidas de proximidade determinam como a similaridade entre dois exemplos é calculada. Sua escolha influencia a forma como os grupos são obtidos e depende dos tipos de atributos que representam os exemplos. Existe uma variedade de medidas de proximidade e as principais adotadas em dados textuais são discutidas na Seção 2.2.1. As estratégias de agrupamento correspondem aos métodos e algoritmos para indução de modelos de agrupamento a partir dos dados (Hastie et al., 2009). As estratégias de agrupamento relacionadas a este trabalho de doutorado são apresentadas na Seção 2.2.2.

\subsubsection{Medidas de Proximidade}

A escolha da medida de proximidade para definir o quão similares são dois exemplos é fundamental para a análise de agrupamentos. Essa escolha depende das características do conjunto de dados, principalmente dos tipos e escala dos dados. Por exemplo, existem medidas de proximidade adequadas para (1) dados contínuos, como Manhanttan, Euclidiana, Pearson e Cosseno; (2) dados binários, como o coeficiente de Jaccard; e (3) mistura entre dados contínuos e binários, como o critério de Gower (Gower, 1971). As medidas de proximidade podem calcular tanto a similaridade quanto dissimilaridade (ou distância) entre exemplos. No entanto, as medidas de similaridade podem ser convertidas para medidas de dissimilaridade e vice-versa (Jain e Dubes, 1988).

A medida de similaridade baseada no cosseno entre dois vetores é a mais utilizada para dados textuais (Aggarwal e Zhai, 2012a), e é definida de acordo com o cosseno do ângulo formado entre os vetores de características de dois exemplos. Para tal, considere $\overrightarrow{x_{i}}=\left(x_{i 1}, x_{i 2}, \ldots, x_{i m}\right)$ e $\overrightarrow{x_{j}}=\left(x_{j 1}, x_{j 2}, \ldots, x_{j m}\right)$ dois exemplos representados em um espaço vetorial $m$-dimensional. A medida de similaridade baseada no cosseno é definida pela Equação 2.1.

$$
\operatorname{cosseno}\left(\overrightarrow{x_{i}}, \overrightarrow{x_{j}}\right)=\frac{\overrightarrow{x_{i}} \cdot \overrightarrow{x_{j}}}{\left\|\overrightarrow{x_{i}}\right\| \| \overrightarrow{x_{j} \|}}=\frac{\sum_{l=1}^{m} x_{i l} x_{j l}}{\sqrt{\sum_{l=1}^{m} x_{i l}^{2}} \sqrt{\sum_{l=1}^{m} x_{j l}^{2}}}
$$

O valor da medida cosseno está no intervalo $[0,1]$ quando aplicada em dados textuais ${ }^{1}$. Assim, se o valor da medida de similaridade cosseno é zero, o ângulo

\footnotetext{
${ }^{1} \mathrm{~A}$ medida de cosseno pode variar no intervalo $[-1,1]$ quando são utilizados valores ne-
} 
entre $\overrightarrow{x_{i}}$ e $\overrightarrow{x_{j}}$ é $90^{\circ}$, ou seja, os documentos não compartilham termos. Por outro lado, se o valor da similaridade for próximo de 1 , o ângulo entre $\overrightarrow{x_{i}} \mathrm{e}$ $\overrightarrow{x_{j}}$ é próximo de $0^{\circ}$, indicando que os documentos compartilham termos e são similares.

Uma técnica que pode ser empregada em agrupamento de textos é a normalização dos vetores de características de cada exemplo (Zhao et al., 2005). Assim, considerando o exemplo $\overrightarrow{x_{i}}=\left(x_{i 1}, x_{i 2}, \ldots, x_{i m}\right)$, diz-se que o vetor está normalizado se for unitário, ou seja, $\|x\|=1$. A normalização pode ser realizada por meio da Equação 2.2.

$$
{\overrightarrow{x_{i}}}^{\text {norm }}=\frac{\overrightarrow{x_{i}}}{\| \overrightarrow{x_{i} \|}}=\frac{\left(x_{i 1}, x_{i 2}, \ldots, x_{i m}\right)}{\sqrt{x_{i 1}^{2}+x_{i 2}^{2}+\ldots+x_{i m}^{2}}}
$$

As principais vantagens em utilizar a normalização dos vetores de características são (1) que a similaridade cosseno pode ser computada apenas pelo produto interno dos vetores de características, $\operatorname{cosseno}\left(x_{i}, x_{j}\right)=x_{i} \cdot x_{j}$, aumentando a eficiência computacional em alguns algoritmos de agrupamento; (2) e que a proximidade entre os exemplos mantém uma relação com a distância euclidiana $d_{e u c}$, na forma $d_{e u c}^{2}\left(\vec{x}_{i}, \vec{x}_{j}\right)=2\left(1-\cos \left(\vec{x}_{i}, \vec{x}_{j}\right)\right)$, permitindo o emprego de algoritmos de agrupamento que possuem propriedades teóricas de convergência com uso de distância euclidiana (Korenius et al., 2007).

Na literatura é apresentada uma variedade de outras medidas de proximidades. No contexto deste trabalho, a medida de similaridade cosseno e sua versão normalizada é suficiente para desenvolvimento das abordagens investigadas nesta tese. Uma revisão mais extensa sobre medidas de proximidade para análise de agrupamentos está disponível nos trabalhos de Everitt et al. (2001) e Tan et al. (2005).

\subsubsection{Métodos de Agrupamento}

Após a escolha de uma medida de proximidade, é selecionado um método para o agrupamento. Os métodos de agrupamento podem ser classificados considerando-se diferentes aspectos. Em geral, as estratégias de agrupamento podem ser organizadas em dois tipos (Rokach, 2010): agrupamento particional e agrupamento hierárquico. No agrupamento particional um conjunto de exemplos é dividido em uma partição simples de $k$ grupos, enquanto no agrupamento hierárquico é produzida uma sequência de partições aninhadas, ou gativos na representação dos atributos. Para dados textuais, geralmente utiliza-se valores baseados em frequência que são maiores ou igual a zero. 
seja, os exemplos são organizados em grupos e subgrupos. Além disso, o agrupamento obtido pode conter sobreposição, isto é, quando um exemplo pertence a mais de um grupo ou, até mesmo, quando cada exemplo possui um grau de pertinência associado aos grupos. No contexto deste trabalho, são exploradas as estratégias que produzem agrupamento sem sobreposição, também conhecidas como estratégias rígidas ou crisp (Everitt et al., 2001; Aggarwal e Reddy, 2013). Assim, se o conjunto de exemplos $X=\left\{\overrightarrow{x_{1}}, \overrightarrow{x_{2}}, \ldots, \overrightarrow{x_{n}}\right\}$ representa uma coleção de $n$ documentos textuais, uma partição rígida $P=\left\{G_{1}, G_{2}, \ldots, G_{k}\right\}$ com $k$ grupos não sobrepostos é tal que:

- $G_{1} \cup G_{2} \cup \ldots \cup G_{k}=X$;

- $G_{i} \neq \emptyset$ para todo $i \in\{1,2, \ldots, k\}$; e

- $G_{i} \cap G_{j}=\emptyset$ para todo $i \neq j$.

As diversas estratégias de agrupamento são, na prática, algoritmos que buscam uma solução aproximada para o problema, dado que ao formular o problema de agrupamento de dados como sendo de otimização combinatória se obtém um problema NP-difícil (Falkenauer, 1998). Enumerar e avaliar todas as possiveis partições é inviável computacionalmente. A seguir, são descritos alguns dos principais algoritmos para agrupamento de textos relacionados a este trabalho de doutorado.

\section{Agrupamento Particional}

No agrupamento particional, o objetivo é dividir o conjunto de exemplos em $k$ grupos, no qual $k$ geralmente é um valor informado previamente pelo usuário. O algoritmo $k$-means (MacQueen, 1967) é o representante mais conhecido para agrupamento particional e muito utilizado em dados textuais. No $k$-means utiliza-se um representante de grupo denominado centroide, que é um vetor médio computado a partir dos vetores do grupo. A Equação 2.3 define o cálculo de um centroide $\vec{c}$ para um determinado grupo $G$, em que $\vec{x}$ representa um exemplo pertencente a $G$ e o número total de exemplos no grupo é $|G|$.

$$
\vec{c}=\frac{1}{|G|} \sum_{\vec{x} \in G} \vec{x}
$$

Dessa forma, o centroide mantém um conjunto de características centrais do grupo, permitindo representar todos os exemplos que pertencem a este grupo. 
Ainda, é importante observar que o $k$-means só é aplicável em situações na qual a média possa ser calculada.

O pseudocódigo para o $k$-means está descrito no Algoritmo 1.

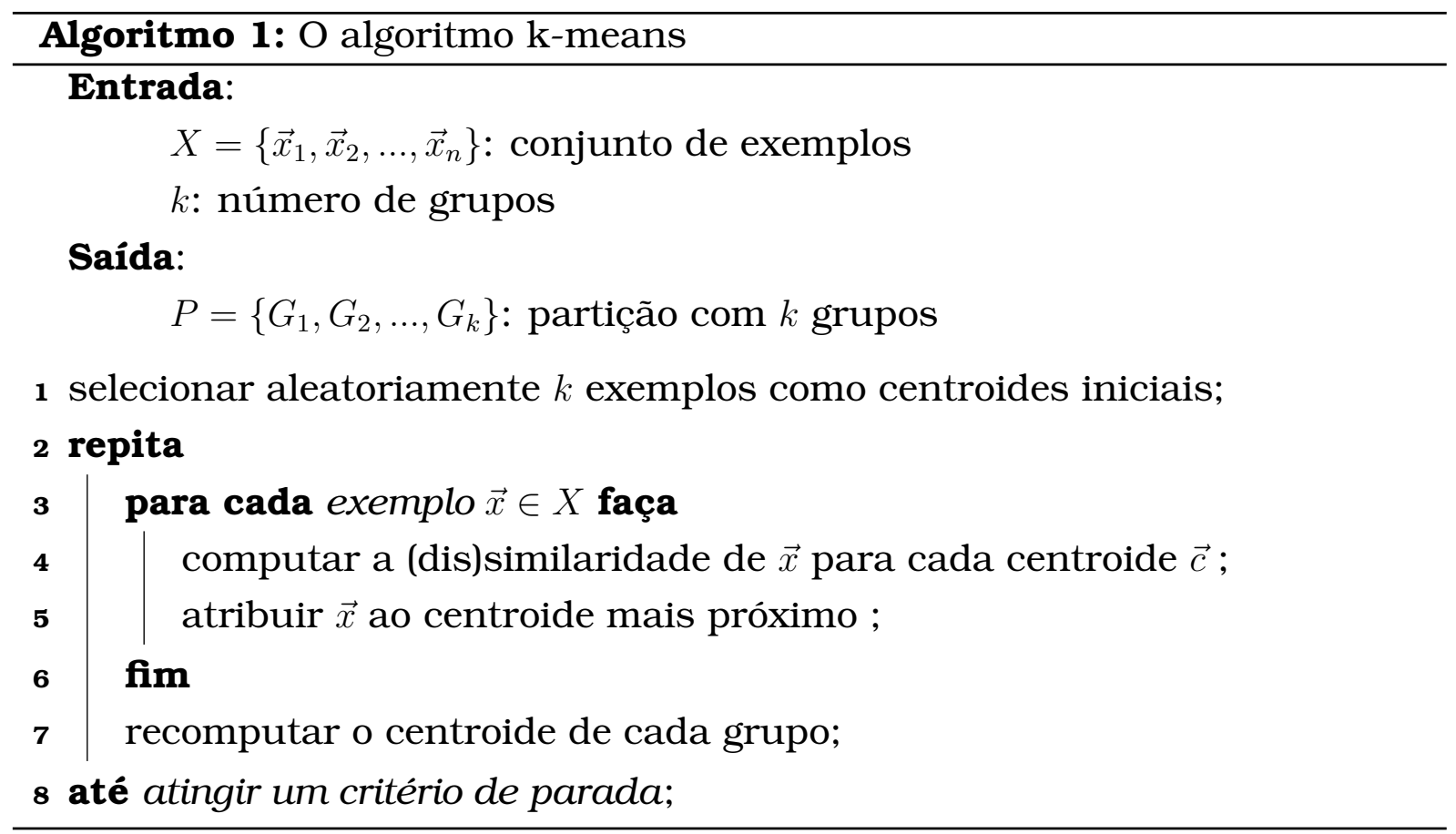

O critério de parada do $k$-means é dado quando não ocorrem mais alterações no agrupamento, ou seja, a solução converge para uma determinada partição. Outro critério de parada prático pode ser um número máximo de iterações.

Durante as iterações do $k$-means, o objetivo é minimizar uma função de erro $E$, definida na Equação 2.4, em que $\vec{x}$ é um exemplo do conjunto de dados; e $\vec{c}_{i}$ é o centroide do grupo $G_{i}$. Observe que é utilizada uma medida de dissimilaridade $\operatorname{dis}\left(\vec{x}, \vec{c}_{i}\right)$ para calcular o valor da função de erro $E$.

$$
E=\sum_{i=1}^{k} \sum_{\vec{x} \in G_{i}} \operatorname{dis}\left(\vec{x}, \vec{c}_{i}\right)
$$

Ao minimizar este critério, o $k$-means tenta separar o conjunto de exemplos diminuindo a variabilidade interna de cada grupo e, consequentemente, aumentando a separação entre os grupos.

A complexidade do $k$-means é linear em relação ao número de exemplos, o que possibilita sua aplicação de modo eficiente em diversos cenários. No entanto, a necessidade de informar com antecedência o número de grupos pode 
ser vista como uma desvantagem, pois esse valor geralmente é desconhecido pelos usuários. Além disso, o método apresenta variabilidade nos resultados, pois a seleção dos centroides iniciais afeta o resultado do agrupamento. Para minimizar esse efeito, o algoritmo pode ser executado diversas vezes, com várias inicializações diferentes, e a solução que apresenta menor valor de erro $E$ é selecionada.

\section{Agrupamento Hierárquico}

Os algoritmos de agrupamento hierárquico podem ser aglomerativos ou divisivos (Xu e Wunsch, 2008). No agrupamento hierárquico aglomerativo, inicialmente cada exemplo pertence a um grupo e, em cada iteração, os pares de grupos mais próximos são unidos até se formar um único grupo. Já no agrupamento hierárquico divisivo, inicia-se com um grupo contendo todos os exemplos que é, então, dividido em grupos menores até restarem grupos unitários (grupos com apenas um exemplo).

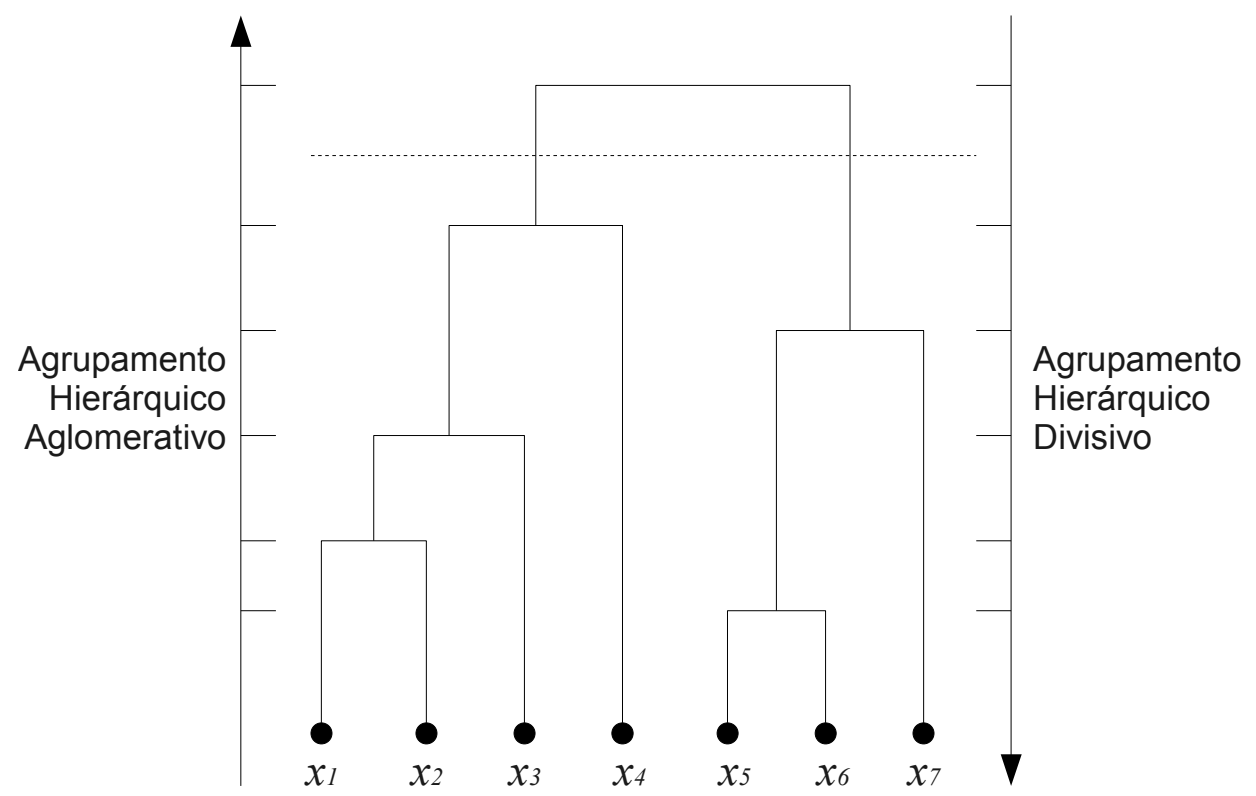

Figura 2.1: Exemplo de um dendrograma (adaptado de Xu e Wunsch (2008))

Tanto os métodos aglomerativos quanto os divisivos organizam os resultados do agrupamento em uma árvore binária conhecida como dendrograma (Figura 2.1). Essa representação é uma forma intuitiva de visualizar e descrever a sequência do agrupamento. Cada nó do dendrograma representa um grupo de exemplos. A altura dos arcos que unem dois subgrupos indica o grau de compactação do grupo formado por eles. Quanto menor a altura, 
mais compactos são os grupos. No entanto, também se espera que os grupos formados sejam distantes entre si, ou seja, que a proximidade de exemplos em grupos distintos seja a menor possível. Essa característica é representada quando existe uma grande diferença entre a altura de um arco e dos arcos formados abaixo dele.

A partir do dendrograma também é possivel obter uma partição com um determinado número de grupos, como nos métodos particionais. Por exemplo, a linha tracejada na Figura 2.1 indica uma partição com dois grupos de exemplos: $\left\{\vec{x}_{1}, \vec{x}_{2}, \vec{x}_{3}, \vec{x}_{4}\right\}$ e $\left\{\vec{x}_{5}, \vec{x}_{6}, \vec{x}_{7}\right\}$.

O pseudocódigo para um algoritmo típico de agrupamento hierárquico aglomerativo está descrito no Algoritmo 2.

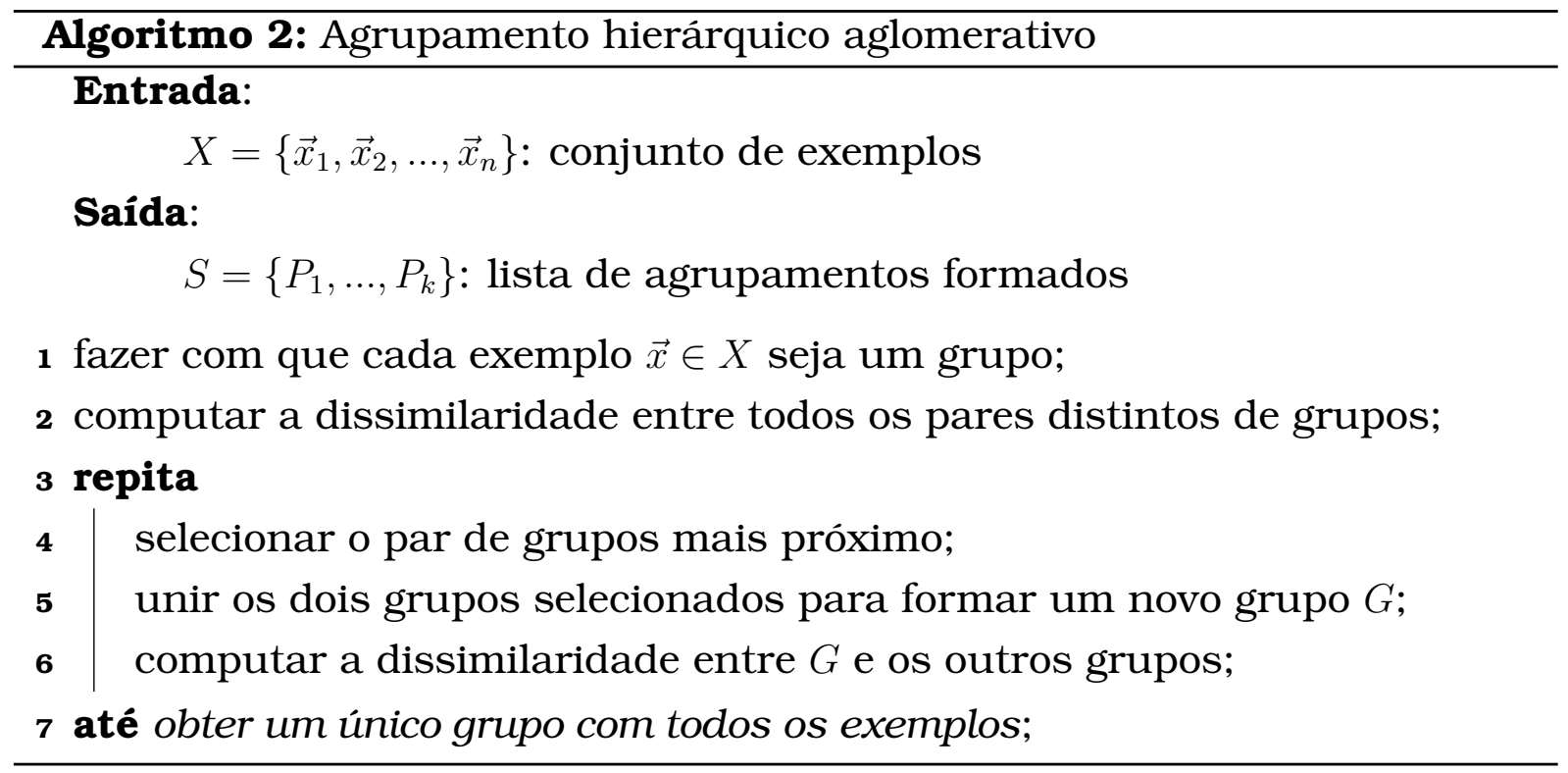

A diferença principal entre os algoritmos de agrupamento hierárquico aglomerativo é o critério de seleção do par de grupos mais próximo (Linha 4 do Algoritmo 2). Os três critérios mais conhecidos são:

- Single-Link (Aggarwal e Reddy, 2013; Sneath, 1957): utiliza o critério de vizinho mais próximo, no qual a distância entre dois grupos é determinada pela distância do par de exemplos mais próximos, sendo cada exemplo pertencente a um desses grupos. Esse método de união de grupos apresenta um problema conhecido como "efeito da corrente", em que ocorre a união indevida de grupos influenciada pela presença de ruídos na base de dados; 
- Complete-Link (Aggarwal e Reddy, 2013; Sorensen, 1948): utiliza o critério de vizinho mais distante. Ao contrário do algoritmo Single-Link, a distância entre dois grupos é a maior distância entre um par de exemplos, sendo cada exemplo pertencente a um grupo distinto. Esse método dificulta a formação do efeito da corrente, como ocorre no Single-Link, e tende a formar grupos mais compactos; e

- Average-Link (Aggarwal e Reddy, 2013; Sokal e Michener, 1958): a distância entre dois grupos é definida como a média das distâncias entre todos os pares de exemplos em cada grupo, cada par composto por um exemplo de cada grupo. Esse método elimina muitos problemas relacionados à dependência do tamanho dos grupos, mantendo próxima a variabilidade interna entre eles.

A escolha do critério de união de grupos dos algoritmos aglomerativos depende geralmente do conjunto de dados e dos objetivos da aplicação. Por exemplo, em dados textuais, avaliações experimentais têm mostrado o AverageLink como a melhor opção entre os algoritmos que adotam estratégias aglomerativas (Zhao et al., 2005).

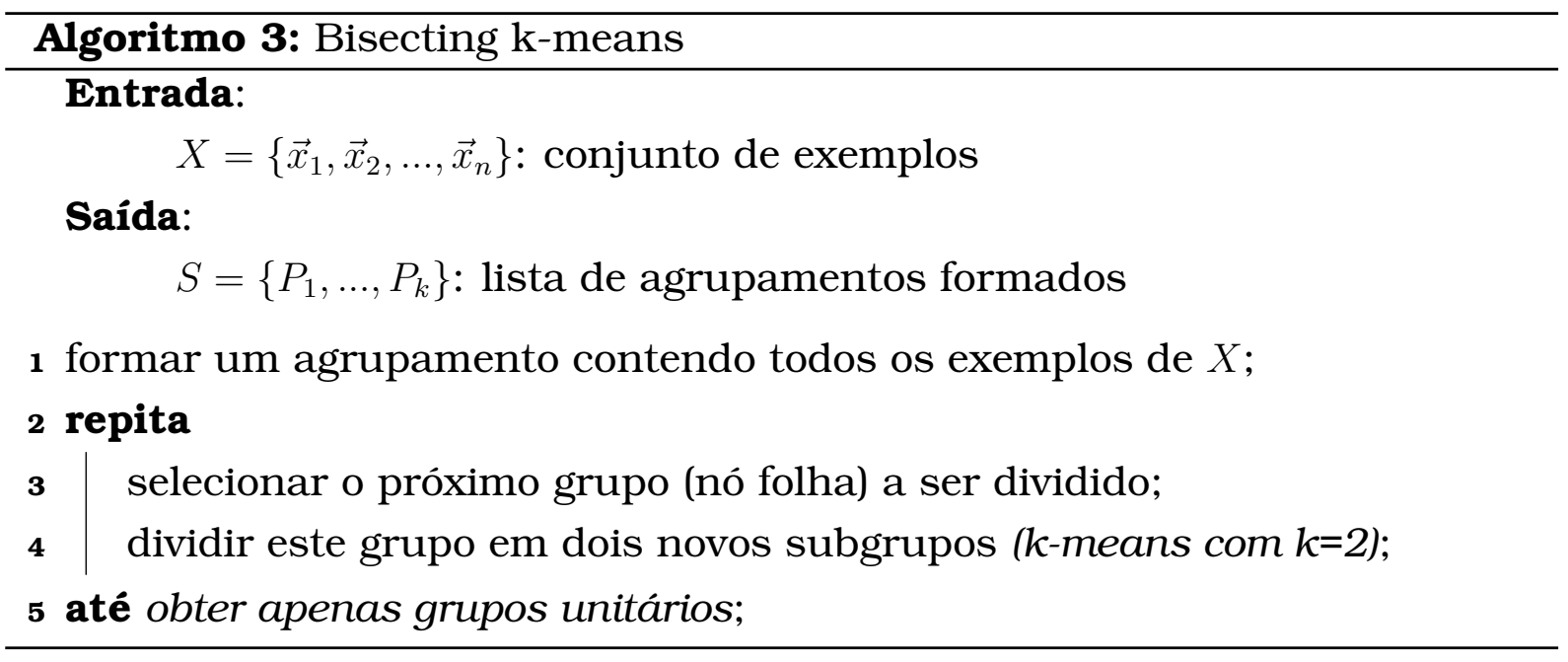

A maioria dos trabalhos relacionados com agrupamento hierárquico na literatura referenciam as estratégias aglomerativas, mostrando pouco interesse nas estratégias divisivas. A possivel causa é a complexidade das estratégias divisivas, que cresce exponencialmente em relação ao tamanho do conjunto de dados, proibindo sua aplicação em grandes conjuntos de dados. Para lidar com esse problema, Steinbach et al. (2000) propuseram o algoritmo Bisecting 
$k$-means, que utiliza agrupamento particional baseado no $k$-means sucessivamente, possibilitando sua aplicação em conjuntos de dados maiores, inclusive em coleções textuais. O pseudocódigo do Bisecting $k$-means está ilustrado no Algoritmo 3.

Existem diferentes maneiras para seleção do próximo grupo a ser dividido (Linha 3 do Algoritmo 3). Um critério simples e eficaz é selecionar o maior grupo (de acordo com o número de exemplos) ainda não dividido em uma iteração anterior (Zhao e Karypis, 2002). Uma propriedade interessante do Bisecting $k$-means é a menor sensibilidade à escolha inicial dos centroides quando comparado com o $k$-means (Tan et al., 2005). Avaliações experimentais indicam que, em dados textuais, o Bisecting $k$-means obtém melhores resultados, seguido do agrupamento hierárquico aglomerativo com o critério Average-Link (Zhao e Karypis, 2002; Zhao et al., 2005).

Os algoritmos de agrupamento hierárquico aglomerativos e divisivos apresentam complexidade (pelo menos) quadrática de tempo e espaço, em relação ao número de exemplos, o que limita o uso direto desses algoritmos para agrupamento de grandes bases de textos. Nesse sentido, diversos trabalhos na literatura exploram o uso de amostragem de dados para lidar com este problema (Xu e Wunsch, 2008; Jain, 2010; Aggarwal e Zhai, 2012a). O diferencial dessas abordagens está relacionado ao fato de que amostras aleatórias de tamanhos apropriados podem preservar importantes propriedades geométricas dos dados, permitindo identificar as principais estruturas de agrupamento com redução de tempo computacional e uso de memória.

Entre as várias estratégias de agrupamento baseadas em amostragem de dados, a abordagem Buckshot Clustering (Cutting et al., 1992; Grossman e Frieder, 2004; Ke et al., 2009; Aggarwal e Zhai, 2012a) é conhecida na área de agrupamento de textos e funciona em duas fases: na primeira fase, um algoritmo de agrupamento hierárquico é aplicado a partir de uma amostra dos dados, obtendo um modelo de agrupamento inicial. Em seguida, novos documentos são incorporados ao modelo utilizando uma estratégia de vizinho mais próximo. Devido a sua simplicidade e eficiência computacional, a abordagem Buckshot Clustering é frequentemente utilizada em análise exploratória de textos e tarefas de recuperação de informação, em aplicações conhecidas como Scatter-Gather (Aggarwal e Zhai, 2012a). 


\section{Agrupamento Considerando Múltiplas Representações}

A diversidade de técnicas para pré-processamento de textos permite a extração de diferentes representações estruturadas para dados textuais. Por exemplo, uma mesma coleção textual pode ser representada por um conjunto de termos, por exemplo, palavras-chave extraídas dos textos, bem como por representações geográficas (utilizando apenas dados georreferenciados identificados nos textos) ou representações específicas de domínio (termos definidos pelos usuários ou ontologias da área). Mais formalmente, em um conjunto de exemplos com duas representações $\chi^{1}$ e $\chi^{2}$, um exemplo $\vec{x}$ é decomposto em $\left(\overrightarrow{x^{1}}, \overrightarrow{x^{2}}\right)$, no qual $\overrightarrow{x^{1}}$ e $\overrightarrow{x^{2}}$ são, respectivamente, vetores no espaço de características em $\chi^{1}$ e $\chi^{2}$ (Bickel e Scheffer, 2004; Kriegel e Zimek, 2010; Hussain et al., 2014).

No trabalho de Bickel e Scheffer (2004) foi proposto uma das primeiras abordagens que exploram agrupamento de textos em múltiplas representações. Para tal, foi proposto e avaliado o $k$-Means para Múltiplas Representações. Em uma primeira etapa, inicializa-se $k$ centroides para uma determinada representação e associa-se cada documento ao seu centroide mais próximo. Assim, os centroides são atualizados e uma partição é obtida. Na próxima iteração, a partição obtida em uma representação é utilizada na outra representação dos dados para uma nova iteração. O processo continua até atingir um determinado número de iterações para cada representação. No final do processo, as partições obtidas em cada representação podem não necessariamente conter os mesmos grupos. Assim, a partição final é calculada por meio de uma média consensual de cada grupo em cada representação. As avaliações experimentais realizadas indicaram que o uso de múltiplas representações melhora o modelo de agrupamento obtido quando comparado com os modelos individuais de cada representação. Um problema crítico deste algoritmo é que não há garantias de convergência, já que alternar as iterações entre diferentes representações torna o processo de agrupamento instável (Bickel e Scheffer, 2004).

Diversos estudos têm demonstrado que combinar diferentes soluções de agrupamento, provenientes de diferentes algoritmos e de múltiplas representações, leva a soluções de maior qualidade (Strehl e Ghosh, 2003; Eaton et al., 2010; Ghosh e Acharya, 2011; Joshi et al., 2014). Uma das motivações desses estudos é que nenhum algoritmo específico é capaz de identificar todos os possiveis formatos e estruturas de grupos existentes em dados reais e em diferentes domínios. Cada algoritmo tem um viés a respeito dos formatos, 
tamanhos e número de grupos, tornando difícil a tarefa de decidir qual o melhor algoritmo para cada tipo de dado.

Nesse contexto, abordagens baseada em consenso de agrupamentos têm ganhado destaque na literatura, pois permitem combinar soluções de diferentes algoritmos de agrupamento em um única solução consensual (Strehl e Ghosh, 2003; Wu et al., 2013; Liu et al., 2014). Os resultados obtidos com consenso de agrupamentos têm sido promissores em vários aspectos. Primeiro, combinar diferentes estruturas de agrupamento, em geral, resulta em uma solução de agrupamento de melhor qualidade do que as soluções individuais. Segundo, consenso de agrupamentos é facilmente paralelizável, promovendo a escalabilidade das aplicações. Por fim, consenso de agrupamentos permite integrar estruturas de agrupamentos obtidos em conjuntos de exemplos que são descritos por múltiplas representações. Os resultados promissores reportados na literatura, em geral, são consequência do princípio de maximização do consenso entre múltiplas hipóteses, que considera que o aprendizado é melhorado utilizando o consenso de todas as representações do conjunto de dados (Bickel e Scheffer, 2004; Long et al., 2008).

Para combinar diferentes soluções de agrupamento é possível empregar uma matriz de coassociação. Nesta matriz, é armazenado o número de vezes que cada par de exemplos está em um mesmo grupo, considerando todas as partições obtidas. A ideia básica é que, se um par de exemplos é frequentemente alocado em um mesmo grupo nas várias partições obtidas, há evidências de que esses exemplos devem ser alocados juntos no agrupamento final. A matriz de coassociação também pode ser vista como uma estratégia de votação, no qual cada partição individual fornece sua opinião a respeito da organização dos dados. Assim, a matriz de coassociação representa um novo conceito de proximidade entre os exemplos que, em geral, é superior à noção de proximidade obtida com medidas de similaridade.

Por fim, a extração do agrupamento consensual é feita aplicando um algoritmo de agrupamento hierárquico na matriz de coassociação. A eficácia da organização dos dados obtida via consenso de agrupamentos, baseada na abordagem aqui descrita, tem sido superior em diversos tipos de aplicações (Ghosh e Acharya, 2011). Em dados textuais, consenso de agrupamentos é potencialmente útil na identificação de estruturas de agrupamento mais robustas, uma vez que documentos erroneamente agrupados em determinadas partições, não necessariamente serão agrupados incorretamente em outras partições, permitindo corrigir esses erros no agrupamento final. 


\section{Agrupamento com Restrições}

Em análise de agrupamentos, uma tarefa é dita bem sucedida se foi possível identificar grupos "naturais" em um conjunto de exemplos. Por outro lado, em problemas reais, os agrupamentos podem possuir diferentes formatos, tamanhos e graus de separação. Ainda, a análise de agrupamentos exige uma noção de proximidade entre exemplos que não é facilmente definida sem ter algum conhecimento a respeito dos dados.

Nesse sentido, foram propostas na literatura os métodos de agrupamento com restrições (Basu et al., 2008). Em geral, há dois tipos de restrições entre os exemplos: Must-Link e Cannot-Link (Wagstaff et al., 2001). Restrições do tipo Must-Link indicam que dois exemplos devem ser alocados em um mesmo grupo. Restrições do tipo Cannot-Link indicam que dois exemplos não devem ser alocados juntos em um grupo. Os algoritmos de agrupamento com restrições devem ajustar o modelo de agrupamento para atender o conjunto de restrições (Wagstaff et al., 2001; Davidson e Ravi, 2005; Basu et al., 2008).

Em geral, os algoritmos buscam (i) satisfazer toda as restrições do fornecidas ou (ii) satisfazer a quantidade máxima de restrições possiveis. O primeiro cenário pode ser definido como agrupamento com hard constraints. No entanto, em muitas aplicações do mundo real, há presença de ruídos nas restrições fornecidas ao algoritmo de agrupamento, principalmente quando o conjunto de restrições é grande (Pelleg e Baras, 2007). Nos casos em que há a necessidade de satisfazer todas as restrições, o processo de agrupamento pode ser intratável levando o algoritmo a retornar uma partição vazia como resposta do agrupamento. Por exemplo, Wagstaff et al. (2001) mostram que uma simples restrição Cannot-Link definida erroneamente é suficiente para comprometer o processo de agrupamento. Em geral, os valores de proximidade entre exemplos são drasticamente distorcidos na presença de algumas poucas restrições ruidosas.

Para superar essas limitações, foram desenvolvidas abordagens mais flexíveis que permitem violar restrições quanto estas comprometem a solução do agrupamento (Bilenko et al., 2004; Law et al., 2004; Davidson e Ravi, 2005; Pelleg e Baras, 2007; Zeng e Cheung, May). Esse cenário é conhecido como agrupamento com soft constraints. Nesse caso, o objetivo é obter a partição que tenha maior concordância com o conjunto de restrições, minimizando o número de restrições violadas. Os resultados de avaliações experimentais reportados na literatura indicam que o agrupamento com soft constraints é mais robusto em cenários com um grande número de restrições, sendo particu- 
larmente interessante em situações em que há uma incerteza associada às restrições fornecidas (Covões et al., 2013).

Os algoritmos que utilizam essa estratégia verificam o quão vantajoso é violar ou não uma determinada restrição. Assim, as restrições que afetam drasticamente as propriedades geométricas dos dados, por exemplo, unindo dois exemplos muito diferentes (ou separando dois exemplos muito semelhantes), estão mais sujeitas a serem violadas durante o processo de agrupamento.

O algoritmo Constrained Vector Guantization Error (CVQE), proposto por Davidson e Ravi (2005), é um dos principais para agrupamento com soft constraints. No algoritmo CVQE são adicionados dois termos na função objetivo do k-means para considerar (i) o custo de violar restrições Must-Link e (ii) o custo de violar restrições Cannot-Link, conforme descrito a seguir:

- Seja $c_{m l}=(a, b)$ uma restrição do tipo Must-link para os exemplos $a$ e $b$. Se esta restrição for violada, então $l_{a} \neq l_{b}$, em que $l_{i}$ é o rótulo do grupo associado ao exemplo $i$. O custo de violar a restrição $c_{m l}=(a, b)$ é dado pelo distância entre os centroides dos grupos $l_{a}$ e $l_{b}$; e

- De forma análoga, seja $c_{c l}=(a, b)$ uma restrição do tipo Cannot-link para os exemplos $a$ e $b$. Se esta restrição for violada, então $l_{a}=l_{b}$. O custo de violar a restrição $c_{c l}=(a, b)$ é dado pela distância entre os centroides dos grupos $l_{a}$ e $l_{n}$, no qual $l_{n}$ é o grupo mais próximo de $l_{a}, \operatorname{com} l_{a} \neq l_{n}$.

Durante as iterações do processo de agrupamento do algoritmo CVQE, os exemplos que não participam de nenhuma restrição são associados ao grupo mais próximo, seguindo o processo convencional do k-means. No entanto, os exemplos que participam de alguma restrição são associados aos grupos considerando o custo de violação dessas restrições. Para tal, são verificadas todas as combinações de associação aos grupos e é selecionada a associação ao grupo que minimiza a função objetivo. Em algumas situações, o custo por violar uma restrição é menor do que o erro quadrático obtido ao manter a restrição, levando o algoritmo CVQE a violar tal restrição.

Uma das principais desvantagens do algoritmo CVQE é o alto custo computacional para verificar todas as combinações de associação ao grupo. Uma variante do CVQE denominado LCVQE (Linear CVQE), proposto por Pelleg e Baras (2007), utiliza uma versão alternativa para verificar os casos em que a restrição pode ser violada, conforme descrito a seguir:

- Inicialmente, todos os exemplos são associados ao grupo mais próximo, seguindo o processo convencional do k-means. 
- Para cada restrição Must-link $c_{m l}=(a, b)$ violada, são verificados três casos para associação aos grupos: (1) verificar o valor da função objetivo ao manter a violação da restrição; (2) verificar o valor da função objetivo ao associar os dois exemplos $a$ e $b$ ao grupo $l_{a}$; e (3) verificar o valor da função objetivo ao associar os dois exemplos $a$ e $b$ ao grupo $l_{b}$. O caso que minimiza a função objetivo é selecionado pelo LCVQE.

- Para cada restrição Cannot-link $c_{c l}=(a, b)$ violada, são verificados dois casos. No primeiro caso, verifica-se o valor da função objetivo ao manter a violação da restrição. No segundo caso, assumindo que $l_{n}$ é o rótulo do grupo associado aos exemplos $a$ e $b$, então o exemplo mais próximo do grupo $l_{n}$ é mantido com seu rótulo atual. O outro exemplo é movido de grupo, sendo associado ao seu grupo vizinho mais próximo. O caso que minimiza a função objetivo é selecionada pelo LCVQE.

Uma extensa avaliação experimental para comparação entre o CVQE e o LCVQE foi apresentada em Covões et al. (2013). O algoritmo LCVQE produz agrupamentos com acurácia semelhante ao CVQE com menor custo computacional. Ainda, o LCVQE obtém soluções de agrupamento com um menor número de violação de restrições do que o CVQE. Por outro lado, o algoritmo CVQE é mais robusto quando há uma grande quantidade de restrições ruidosas.

Outra estratégia interessante é apresentada no algoritmo MPC-Kmeans (Bilenko et al., 2004), que utiliza o conjunto de restrições para aprender uma medida de proximidade. Nesse caso, a nova medida de proximidade busca respeitar as restrições indicando alta proximidade entre pares de exemplos pertencentes ao conjunto Must-Link e baixa proximidade entre pares de exemplos do conjunto Cannot-Link. Esta estratégia de agrupamento com aprendizado de métricas é discutida de forma detalhada no Capítulo 4 .

\section{Agrupamento Incremental}

O agrupamento incremental é baseado no pressuposto de que é possível observar um exemplo de cada vez e alocá-lo a um agrupamento existente (Maimon e Rokach, 2005). De uma maneira mais formal, dada uma sequência de exemplos $\overrightarrow{x_{1}}, \overrightarrow{x_{2}}, \ldots, \overrightarrow{x_{n}}$, é possível construir um agrupamento $P_{h+1}$, com base apenas no agrupamento anterior $P_{h}$ e no exemplo atual $\overrightarrow{x_{i}}$ (Giraud, 2000). Ainda, os algoritmos de agrupamento incremental não devem armazenar todos os exemplos em memória, mas apenas os representantes de cada grupo 
existente (Xu e Wunsch, 2008). Em geral, os requisitos de tempo e espaço dos algoritmos de agrupamento incremental são pequenos (Maimon e Rokach, 2005), o que os tornam úteis para extração de conhecimento em grandes bases de dados e em cenários dinâmicos.

O algoritmo Leader-Follower (Hartigan, 1975; Jain et al., 1999) é um dos métodos de agrupamento incremental mais simples e comumente utilizado (Xu e Wunsch, 2008). Neste algoritmo, para cada novo exemplo apresentado compara-se sua proximidade com os grupos existentes. Se o valor da proximidade respeitar um limiar definido pelo usuário, então o exemplo é alocado ao grupo existente mais próximo. Caso contrário, um novo grupo é criado para o exemplo. Em geral, usa-se o centroide dos grupos para o cálculo da proximidade com novos exemplos. Ainda, quando um exemplo é alocado a um grupo existente, o respectivo centroide é ajustado. É importante observar que o ajuste dos centroides é realizado de forma eficiente com equações específicas para cálculo incremental de médias (Finch, 2009).

Entre os trabalhos existentes na literatura que investigam o agrupamento incremental de textos, pode-se destacar o Topic Detection and Tracking (Allan, 2002; Dai et al., 2010). Essa abordagem surgiu da necessidade de agrupar notícias de assuntos relacionados, facilitando o acesso à informação e diminuindo a redundância de conteúdo. Para realização desta tarefa, foram propostas adaptações dos tradicionais algoritmos de agrupamento baseado em similaridades entre documentos. De uma maneira geral, o agrupamento incremental é realizado pela adição de cada novo documento ao grupo mais similar (Hammouda e Kamel, 2003). Quando a similaridade entre o documento apresentado e os grupos existentes não atende a um valor definido, um novo grupo é formado e o documento é alocado a esse grupo. Uma desvantagem desses métodos é a grande sensibilidade à ordem de inserção de documentos, ou seja, o agrupamento final é dependente da ordem em que os documentos são adicionados, provocando alta variabilidade nos resultados.

No trabalho de Zhang et al. (1996), é proposto o método Birch (Balanced Iterative Reducing and Clustering using Hierarchies). Os autores apresentam um algoritmo que atualiza incrementalmente um sumário com as principais informações da base de dados, sem a necessidade de armazenar todos os exemplos em memória. A estrutura responsável por manter esse sumário é denominada Cluster Feature (CF), que é organizada em uma árvore chamada CF-Tree. Quando um novo exemplo é apresentado, realiza-se uma busca pelo nó folha com a CF mais similar ao exemplo, partindo-se da raiz. Ao encontrar 
um nó folha, o exemplo é inserido e as informações da CF são atualizadas, assim como as CF's dos nós ancestrais. Zhang et al. (1996) afirmam que os nós folhas da CF-Tree podem ser, então, agrupados por um algoritmo de agrupamento tradicional para obtenção de uma organização dos dados. Originalmente, o Birch foi proposto para trabalhar com bases de dados nas quais medidas métricas podem ser aplicadas. Uma adaptação desta abordagem considerando dados textuais é apresentada no trabalho de Wang e Li (2010).

\subsubsection{Avaliação de Agrupamentos}

A validação do resultado de um agrupamento, em geral, é realizada por meio de índices estatísticos que expressam o "mérito" das estruturas encontradas, ou seja, quantificam alguma informação sobre a qualidade de um agrupamento (Faceli et al., 2005; Xu e Wunsch, 2008). O uso de técnicas de validação em resultados de agrupamento é uma atividade importante, uma vez que algoritmos de agrupamento sempre encontram grupos nos dados, independentemente de serem reais ou não (Halkidi et al., 2001).

As estratégias utilizadas no contexto de validação e seleção de modelos de agrupamento, como identificar o melhor número de grupos, são discutidas em mais detalhes no Capítulo 5. Nesta seção, é apresentado um índice de avaliação comumente utilizado nos trabalhos relacionados a agrupamento hierárquico de textos (Zhao et al., 2005), denominado FScore (Larsen e Aone, 1999).

O índice FScore é uma medida que utiliza as ideias de precisão e revocação, da recuperação de informação, para avaliar a eficácia de recuperação em agrupamentos hierárquico de textos (Larsen e Aone, 1999; Zhao e Karypis, 2002; Tan et al., 2005). É empregado como critério externo de validação, pois utiliza o conhecimento prévio (informação externa) sobre categorias ou tópicos existentes no conjunto de dados. A ideia básica é verificar o quanto o agrupamento hierárquico conseguiu reconstruir a informação de categoria associada a cada exemplo.

Para o cálculo do índice FScore, considere que

- $H$ é um agrupamento hierárquico obtido (dendrograma) por um determinado algoritmo;

- $L_{r}$ é uma determinada categoria (informação externa) representando um conjunto de exemplos de um mesmo tópico ou classe; e 
- $G_{i}$ é um determinado grupo, e seu respectivo conjunto de exemplos, pertencente ao agrupamento hierárquico $H$.

Assim, dada uma categoria $L_{r}$ e um grupo $G_{i}$, calcula-se as medidas de precisão $P$ e revocação $R$ conforme a Equação 2.5 e Equação 2.6, respectivamente. Em seguida, é obtida a média harmônica $F$ (Equação 2.7), que representa um balanceamento entre a precisão e revocação.

$$
\begin{gathered}
P\left(L_{r}, G_{i}\right)=\frac{\left|L_{r} \cap G_{i}\right|}{\left|G_{i}\right|} \\
R\left(L_{r}, G_{i}\right)=\frac{\left|L_{r} \cap G_{i}\right|}{\left|L_{i}\right|} \\
F\left(L_{r}, G_{i}\right)=\frac{2 * P\left(L_{r}, G_{i}\right) * R\left(L_{r}, G_{i}\right)}{P\left(L_{r}, G_{i}\right)+R\left(L_{r}, G_{i}\right)}
\end{gathered}
$$

A medida $F$ selecionada para uma determinada categoria $L_{r}$ é o maior valor obtido por algum grupo da hierarquia $H$, considerando todas os grupos e subgrupos existentes, conforme a Equação 2.8.

$$
F\left(L_{r}\right)=\max _{G_{i} \in H} F\left(L_{r}, G_{i}\right)
$$

Finalmente, o valor FScore global de um agrupamento hierárquico com $n$ exemplos e $c$ categorias, é calculado como o somatório da medida $F$ de cada categoria ponderada pelo número de exemplos da categoria (Equação 2.9).

$$
\text { FScore }=\sum_{r=1}^{c} \frac{\left|L_{r}\right|}{n} F\left(L_{r}\right)
$$

Conforme o agrupamento hierárquico consegue reconstruir a informação das categorias predeterminadas de uma base de textos, o valor de FScore se aproxima de 1 . Caso contrário, a FScore tem valor 0 . Observe que essa medida trata cada grupo da hierarquia como se fosse o resultado de uma consulta e cada categoria predefinida da coleção como o conjunto de exemplos relevantes para essa consulta.

\subsection{Aprendizado de Máquina com Informação Privilegiada}

Em um paradigma tradicional de agrupamento de dados, o conjunto de exemplos $X=\left\{\vec{x}_{1}, \ldots, \vec{x}_{n}\right\}$ é representado por um único espaço de característi- 
cas $\chi$. Por outro lado, em muitos domínios há informação adicional a respeito do conjunto de exemplos $X$ que não está refletida no espaço de características $\chi \mathrm{e}$, consequentemente, esta informação adicional não é considerada durante a obtenção dos modelos.

Recentemente, um crescente número de estudos tem investigado métodos para obtenção de modelos considerando dados com múltiplas representações (Blum e Mitchell, 1998; Bickel e Scheffer, 2004; Ruping e Scheffer, 2005; Zhou e Burges, 2007; Zhang et al., 2008; Long et al., 2008; Kim et al., 2010; Zhuang et al., 2012). A principal motivação desses estudos está baseada no princípio de maximização do consenso entre múltiplas hipóteses, que considera que o consenso entre modelos individuais obtidos em cada representação tende a obter um modelo final superior aos modelos individuais (Dasgupta et al., 2001; Abney, 2002). A seguir são descritos alguns exemplos de aplicações que motivam esses trabalhos, tanto para obtenção de modelos de agrupamento quanto de classificação.

- Páginas na web são um problema típico sobre dados com múltiplas representações. Os exemplos (páginas) podem ser descritos em vários espaços de características, como o texto contido nas páginas (estrutura bag-ofwords), a rede formada pelas âncoras entre as páginas e, até mesmo, imagens existentes nas páginas (bem como outros recursos de multimídia). A base de dados de benchmark conhecida como $\mathrm{WebKB}^{2}$ é frequentemente utilizada para avaliar este tipo de problema, no qual duas representações, textos e rede de âncoras, são combinadas para obtenção de modelos mais eficazes.

- A análise do conhecimento implícito em bases de dados composta por artigos científicos é uma aplicação que tem recebido atenção na literatura. Neste tipo de aplicação, as representações são o texto dos artigos e a rede de citação entre os artigos. Em análise de comunidades científicas é possivel explorar uma representação formada pela rede de coautoria e a representação formada pelo perfil textual (palavras-chave indicando áreas de pesquisa) dos autores.

- Em sistemas de recuperação de imagens, o conjunto de exemplos pode ser descrito por diversos espaços de características provenientes das propriedades das imagens, como cor, textura e forma. Além disso, aplicações

\footnotetext{
${ }^{2}$ WebKB: http://www.cs.purdue.edu/commugrate/data_access/all_data_sets.php
} 
para compartilhamento de imagens na $w e b$, como o Flickr ${ }^{3}$, permitem a anotação de imagens por meio de tags e, assim, também há uma representação textual disponível para os exemplos.

- Na área de segmentação de vídeos, um dos objetivos é identificar, extrair e organizar cenas ou tomadas dos respectivos vídeos. O conjunto de exemplos é representado tanto pelas características das imagens dos vídeos, quanto pelas características sonoras (e.g. detecção de silêncio), e até por conteúdo textual (closed caption).

- Em análise de redes sociais são explorados padrões em redes de amizades, como detecção de comunidades e predição de links. O conjunto de exemplos é formado pelos usuários das redes e este conjunto é descrito por meio de um grafo. Em estudos mais recentes, novas representações dos dados têm sido incorporadas nessas análises - por exemplo, informações geográficas dos usuários e a informação textual (comentários e opiniões) que os usuários publicam nas redes. Como resultado, a análise de redes sociais considerando múltiplas representações aumentam a eficácia de sistemas de recomendação, bem como a eficácia da tarefa de detecção de comunidades.

A existência de múltiplas representações dos dados leva a um relevante desafio ainda pouco explorado: como lidar com diferentes níveis de importância e de disponibilidade das várias representações de um conjunto de exemplos durante a obtenção dos modelos? Abordagens que assumem que as múltiplas representações do conjunto de exemplos são igualmente importantes e que todos os exemplos estão representados nessas representações podem facilmente falhar em aplicações do mundo real. Para exemplificar, considere o problema de agrupamento de imagens, no qual os exemplos são representados por meio de (i) características extraídas a partir dos pixels das imagens e (ii) pela descrição textual (tags) sobre exemplos presentes nas imagens. É natural afirmar que, em uma grande base de imagens, um número considerável de exemplos não tenha descrição textual associada. Por outro lado, as representações textuais permitem descrever os exemplos em um nível semântico que dificilmente é alcançado pelas características extraídas dos pixels das imagens, ou seja, é desejável definir um valor de importância diferente para as descrições para a composição do modelo final.

\footnotetext{
${ }^{3}$ Flickr: http://www.flickr.com
} 


\subsubsection{O paradigma LUPI (Learning using Privileged Information)}

O paradigma LUPI (Learning using Privileged Information) foi proposto originalmente por Vapnik e Vashist (2009) para obtenção de modelos preditivos (classificação e regressão) a partir de exemplos com múltiplas representações. O diferencial do paradigma LUPI é a estratégia utilizada para lidar com o problema de disponibilidade e importância das representações. No paradigma LUPI é necessário organizar as representações do conjunto de exemplos em dois tipos, conforme descrito a seguir.

1. Informação Técnica: dados gerados a partir de um processo técnico, de baixo custo (recursos humanos e computacionais) e altamente disponível para descrever os exemplos. Devido a esta alta disponibilidade, as representações originadas de informação técnica geralmente são o alvo para um processo de aprendizado de máquina.

2. Informação Privilegiada: dados gerados a partir de um processo avançado, de alto custo, dependente de domínio, e que representa uma informação adicional, potencialmente valiosa, a respeito dos exemplos. Devido ao alto custo para obtenção desses dados, a informação é definida como privilegiada e usualmente está disponível para representar apenas um pequeno subconjunto dos exemplos.

O seguinte cenário foi descrito por Vapnik e Vashist (2009) para ilustrar a diferença entre informação técnica e informação privilegiada. Suponha um problema de classificação de imagens de biópsia em duas classes: câncer e não câncer. Uma pequena parcela das imagens foi classificada manualmente por um especialista para permitir o aprendizado de um modelo preditivo. Assim, o conjunto de exemplos $X$ é dividido em dois subconjuntos $X_{c}$ e $X_{\bar{c}}$, que são respectivamente os subconjuntos de exemplos manualmente classificados e os não classificados, com $X=X_{c} \cup X_{\bar{c}}$ e $X_{c} \cap X_{\bar{c}}=\emptyset$. Todos os exemplos estão representados no espaço de características $\chi$, que são informações visuais extraídas automaticamente dos pixels das imagens. Por outro lado, apenas o subconjunto de exemplos manualmente classificados $X_{c}$ possui uma segunda descrição $\chi^{*}$, formado pelo diagnóstico textual emitido por um patologista, com a análise, comentários e observações sobre a imagem da biópsia. Em resumo, o modelo preditivo é obtido a partir de

$$
X_{c}=\left\{\left(\vec{x}_{1}, \vec{x}_{1}^{*}, y_{1}\right), \ldots,\left(\vec{x}_{m}, \vec{x}_{m}^{*}, y_{m}\right)\right\}, \quad \vec{x}_{i} \in \chi, \quad \vec{x}_{i}^{*} \in \chi^{*}, \quad y_{i} \in\{\text { cancer,nao_cancer }\},
$$


no qual $\chi$ representa o espaço de características definido como informação técnica e $\chi^{*}$ representa a informação privilegiada. O modelo deve classificar os exemplos do subconjunto

$$
X_{\bar{c}}=\left\{\vec{x}_{m+1}, \ldots, \vec{x}_{n}\right\}, \quad \vec{x}_{i} \in \chi,
$$

que é descrito apenas pelas informações visuais dos pixels das imagens, ou seja, pela informação técnica $\chi$. Este cenário é útil para ilustrar as seguintes diferenças entre informação técnica e informação privilegiada:

- O espaço de características $\chi$ proveniente de informação visual extraída dos pixels das imagens, neste caso, é um exemplo de informação técnica, pois é originado a partir de um processo técnico, relativamente de baixo custo, baseado em pré-processamento de imagens. É uma representação facilmente acessível e que pode ser extraída automaticamente para futuras imagens, o que motivou a extração de um modelo preditivo para classificação dessas imagens.

- O espaço de características $\chi^{*}$ é um tipo de informação privilegiada, uma vez que envolve um alto custo para obtê-la (especialista da área). Ainda, esta representação está disponivel apenas para o subconjunto de exemplos manualmente classificados, o que permite usá-la somente durante a obtenção do modelo preditivo. Neste caso, a ideia é que o modelo obtido pela combinação de $\chi$ e $\chi^{*}$ seja melhor do que o modelo obtido apenas a partir de $\chi$. O modelo deve ser capaz de classificar as imagens do conjunto $X_{\bar{c}}$, que possui apenas a representação composta pelas características extraídas dos pixels das imagens.

- É importante ressaltar que a informação privilegiada utiliza o mesmo conjunto de rótulos/classes da informação técnica, ou seja, é um novo espaço de características para os exemplos. Além disso, assim como a informação técnica, a informação privilegiada não é “perfeita”, e está sujeita aos mesmos problemas das outras representações, como ruídos e alta dimensionalidade.

Embora seja possivel realizar o aprendizado usando apenas a informação técnica, o aprendizado pode ser melhorado na presença das explicações, comentários e comparações a respeito dos dados. Vapnik e Vashist (2009) afirmam que em praticamente todos os domínios há algum tipo de informação 
privilegiada que pode ser incorporada em tarefas de aprendizado. Neste sentido, uma importante questão de pesquisa é: como incorporar informação privilegiada durante a obtenção de modelos? Na próxima seção são apresentados alguns algoritmos e aplicações existentes na literatura para esta finalidade.

\subsubsection{Algoritmos e Aplicações baseados no paradigma LUPI}

Após a introdução do paradigma LUPI no trabalho de Vapnik e Vashist (2009), diversos outros estudos têm surgido na literatura, investigando informação privilegiada em tarefas de aprendizado de máquina. Na Figura 2.2 é ilustrado o número de publicações (artigos em conferências e periódicos) sobre esse assunto de 2009 a maio de 2014. Nesses trabalhos estão incluídos aqueles relacionados aos aspectos teóricos do LUPI, algoritmos, aplicações e até mesmo os que comentam empregar o paradigma LUPI como possiveis direções de pesquisa.

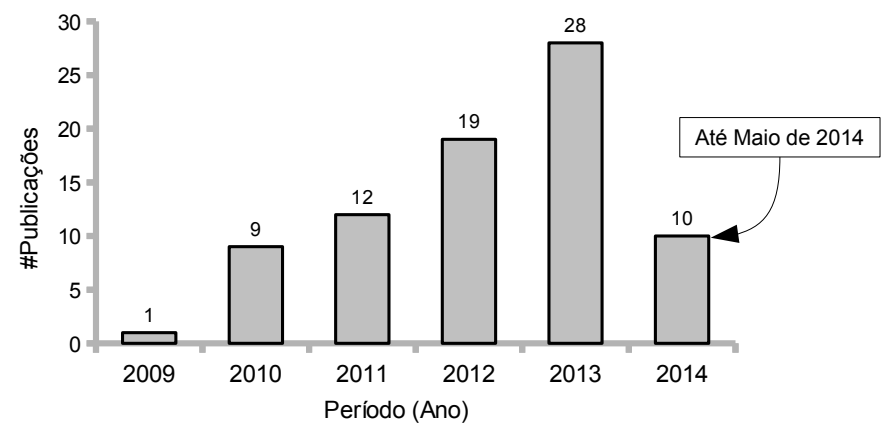

Figura 2.2: Visão geral do número de publicações que discutem ou investigam a abordagem LUPI (Learning Using Privileged Information) de 2009 a maio de 2014.

Foram selecionados aproximadamente $20 \%$ dos trabalhos mais relacionados ao contexto deste trabalho de doutorado para serem descritos nesta seção. O objetivo é dar uma visão geral de como o paradigma LUPI tem sido empregado ao longo dos anos, identificar lacunas de pesquisas e justificar as abordagens exploradas neste trabalho.

Vapnik e Vashist (2009): neste trabalho é introduzido o paradigma LUPI (Learning Using Privileged Information) para aprendizado supervisionado. É proposta uma extensão do algoritmo $S V M$, denominada $S V M+$, para incorporar informação privilegiada durante o treinamento de modelos de classificação e regressão. Também é proposta uma abordagem para 
incorporar informação privilegiada considerando o conceito de aprendizado de métricas. Nesse caso, as relações de proximidades existentes entre exemplos no espaço de informação privilegiada são incorporadas na medida de proximidade a ser empregada no espaço de informação técnica. Entre as avaliações realizadas para verificar a eficácia do paradigma LUPI, está o problema de classificação de proteínas, em que o objetivo é identificar a família de uma determinada proteína. Em geral, é possivel apoiar essa classificação utilizando uma representação baseada na estrutura 3D da proteína. No entanto, extrair tal representação é extremamente complexo, dependente de especialistas e ainda desconhecido para alguns tipos de proteína. Por outro lado, extrair a sequência de aminoácidos da proteína é uma tarefa mais fácil e proveniente de um processo técnico, o que torna esta representação frequentemente utilizada para classificação de proteínas. Na avaliação experimental, a representação 3D da proteína é utilizada como informação privilegiada, enquanto a representação baseada na sequência de aminoácidos é utilizada como informação técnica. Na avaliação experimental foram analisados 80 problemas de classificação de proteínas, no qual 66 apresentaram melhorias na acurácia de classificação com a incorporação de informação privilegiada. Entre os 14 restantes, o paradigma LUPI obteve resultados similares aos paradigmas tradicionais de classificação.

Pechyony e Vapnik (2010, 2011); Cai e Cherkassky (2012): no trabalho de Pechyony e Vapnik (2010) é realizado um estudo teórico do paradigma LUPI para aprendizado supervisionado, em especial, no algoritmo $S V M+$. Os autores demonstram que a informação privilegiada pode ser utilizada para aumentar a taxa de aprendizado do modelo, em particular, para encontrar hiperplanos mais adequados que separam as classes do problema. Um resultado interessante desse estudo é que o paradigma LUPI pode encontrar melhores soluções mesmo quando a informação privilegiada contém ruídos. Os algoritmos relacionados a este estudo téorico são apresentados em Pechyony e Vapnik (2011), que envolvem estratégias para implementação do classificador $S V M+$ para classificação binária. Mais recentemente, Cai e Cherkassky (2012) discutem estratégias eficientes de implementação do classificador $S V M+$ considerando problemas multiclasses, resultado da tese de doutorado de Feng Cai (2011).

Ribeiro et al. (2010, 2012): no trabalho de Ribeiro et al. (2010), o problema 
de previsão de falências de empresas foi investigado por meio do paradigma LUPI. Os autores afirmam que a taxa de falência aumentou nos últimas anos e está se tornando mais difícil de estimar conforme as relações empresariais se tornam mais complexas. Apesar de uma grande variedade de técnicas aplicadas ao longo dos anos, nenhum método que permitia incluir diferentes representações até então tinha sido considerado nos modelos preditivos. Em vista disso, os autores utilizaram o classificador $S V M+$ para a previsão de falências de empresas. Foi selecionado um conjunto de dados sobre 1200 empresas francesas, representadas por vários indicadores financeiros (informação técnica) a respeito de 5 anos de operação (2002 - 2006). A informação privilegiada foi composta por análises financeiras de especialistas. Os resultados experimentais indicam um aumento significativo da acurácia do modelo com uso do $S V M+$ comparado a modelos que não incorporam informação privilegiada. Em especial, há uma diminuição drástica de erros do tipo "falso negativo", ou seja, quando uma empresa com grande probabilidade de falência é classificada como viável. Uma extensão deste trabalho é apresentada em Ribeiro et al. (2012).

Feyereisl e Aickelin (2012): o uso do paradigma LUPI para tarefas de agrupamento foi investigado neste trabalho, sendo um resultado da tese de doutorado de Jan Feyereisl (2010). Os autores propuseram a abordagem aRi-MAX (Maximum Adjusted Rand Index), que utiliza informação privilegiada para obter um conjunto de modelos de agrupamento (partições) de referência. Em seguida, são obtidas diversas partições a partir da informação técnica. A abordagem aRi-MAX realiza, então, um processo de seleção de modelos. O objetivo é identificar um modelo de agrupamento proveniente da informação técnica que tenha alta correlação com algum modelo de agrupamento de informação privilegiada. Para tal, o índice Rand (Hubert e Arabie, 1985) é utilizado para comparar dois modelos de agrupamento. A abordagem aRi-MAX foi avaliada em conjuntos artificiais e com diversas execuções do $k$-means. Neste caso, a informação privilegiada do problema consiste de regras sobre a distância entre exemplos de um mesmo grupo. A informação técnica consiste de coordenadas no plano cartesiano dos exemplos a serem agrupados. Os resultados indicam que a abordagem aRi-MAX permite empregar a informação privilegiada para selecionar modelos de agrupamento mais robustos, quando comparado com agrupamento baseado apenas em informação técnica. 
A abordagem aRi-MAX é descrita em mais detalhes no Capítulo 5 desta tese.

Fouad et al. (2012, 2013): no trabalho de Fouad et al. (2012), os autores propuseram o uso de aprendizado de métricas como estratégia para incorporar informação privilegiada em algoritmos de classificação. Em um esquema de classificação baseado em protótipos, no qual cada um dos protótipos está associado a uma classe, um exemplo de teste é classificado de acordo com a classe do protótipo mais similar. Os protótipos são obtidos, inicialmente, considerando a representação de informação técnica. Em seguida, as relações de proximidades existentes no espaço de informação privilegiada são extraídas e utilizadas para recalcular a importância de cada característica (atributo) dos protótipos. Dessa forma, ao calcular a similaridade entre um exemplo de teste e um protótipo, o cálculo de proximidade leva em consideração a informação privilegiada do problema. Este trabalho foi estendido para outros classificadores baseado em distâncias em Fouad et al. (2013). Nas avaliações experimentais realizadas, a tarefa de classificação utilizando a medida de distância com informação privilegiada obteve melhores resultados de classificação do que usando apenas informação técnica. Esses resultados motivaram o trabalho sobre incorporar informação privilegiada em aprendizado de métricas para tarefas de agrupamentos, detalhado no Capítulo 4 (Informação Privilegiada em Aprendizado de Métricas).

Yang e Patras (2013): neste trabalho os autores exploram o paradigma LUPI para melhorar o estado da arte na área de detecção de características faciais (identificação de partes da face). Os conceitos propostos no $S V M+$ foram utilizados em uma abordagem chamada "Regression Forests", que emprega um comitê de árvores de decisão para detecção de características faciais. Nesse caso, a informação privilegiada é utilizada para melhorar o critério de seleção de nós internos da árvore. Como informação privilegiada, os autores exploraram informações adicionais do problema que só estão disponíveis na fase de treinamento, como o ângulo da cabeça, expressão facial e o sexo da pessoa na imagem. A informação técnica consiste de atributos extraídos pelo pré-processamento das imagens. Os autores utilizam dois conjuntos de dados de benchmark para comparar a abordagem proposta com algoritmos que são estado-da-arte na área, e os resultados indicam que o uso da informação privilegiada melhorou 
significativamente a detecção de características faciais.

Sharmanska et al. (2013): neste trabalho os autores investigam o problema de classificação de imagens com uso do paradigma LUPI. Neste caso, foram explorados diversos tipos de informação privilegiada para as imagens, como tags para informação contextual, anotações semânticas sobre propriedades da imagem e caixas delimitadoras que focam objetos de interesse na imagem. A avaliação experimental foi realizada em conjunto de dados para classificação de diferentes espécies de animais. Além da análise do $S V M+$ para a tarefa de classificação, também foi avaliado o ranking produzido com base na confiança de classificação. O paradigma LUPI, para este cenário, foi comparado com os algoritmos equivalentes sem uso de informação privilegiada. Na maioria das avaliações, os autores reportam um aumento significativo da acurácia de classificação. No pior cenário, o uso de paradigma LUPI foi equivalente aos classificadores tradicionais.

Serra-Toro et al. (2014): uma análise experimental da eficácia do paradigma LUPI para aprendizado supervisionado, em particular, do classificador $S V M+$ é discutida neste trabalho. Os autores discutem sobre alguns aspectos práticos que determinam quando a informação privilegiada utilizada para o problema pode ser útil. Os autores reproduziram os experimentos reportados em Vapnik e Vashist (2009) e, adicionalmente, substituiram a informação privilegiada original por uma representação composta por atributos aleatórios. O algoritmo $S V M+$ com informação privilegiada original do problema ainda obtém resultados superiores em comparação aos cenários alternativos analisados, porém, a incorporação de atributos aleatórios também apresenta um aumento da acurácia quando comparado com o tradicional $S V M$. Este resultado foi explicado pelo fato de que a segunda representação dos dados também tem a importante função de reduzir um super ajuste do modelo aos dados durante o treinamento ("overfitting"). Em particular, este efeito é mais aparente quando o conjunto de dados de treinamento é relativamente pequeno, cenário em que o uso de informação privilegiada se mostrou mais promissor.

Lapin et al. (2014): neste trabalho é realizada uma comparação teórica entre o classificador $S V M+$ e o classificador WSVM (Weighted SVM) (Lauer e Bloch, 2008). No classificador $W S V M$, assume-se que cada exemplo do 
conjunto de treinamento possui um peso que determina sua importância. Os pesos são geralmente fornecidos por especialistas do domínio do problema. Os autores apresentam uma discussão teória da conexão entre essas duas abordagens, indicando que o $S V M+$ pode ser visto como um caso especial do WSVM. Neste caso, a informação privilegiada pode ser empregada de forma indireta para determinar a importância dos exemplos para o classificador $W S V M$. Por outro lado, os autores afirmam que em problemas que possuem naturalmente múltiplas representações, o uso do $S V M+$ é mais indicado.

Qi et al. (2014): neste trabalho os autores incorporam informação privilegiada para o problema de rastreamento visual de exemplos. O problema consiste em reconhecer um determinado exemplo que está em movimento em várias imagens consecutivas. Tradicionalmente, o problema é tratado como classificação binária tentando reconhecer se o exemplo está ou não presente em determinados locais da imagem. Durante o treinamento, várias partes das imagens são utilizadas para compor exemplos positivos e negativos para treinamento de um modelo de classificação. Nesses casos, o aspecto temporal (exemplo em várias imagens) não é considerada. Os autores utilizam as imagens "futuras" do exemplo dentro do conjunto de treinamento como informação privilegiada do problema para refinar o modelo de classificação. Durante a etapa de teste, há apenas o exemplo e a imagem atual (informação técnica). Os resultados experimentais reportados indicam que modelos obtidos com apoio da informação privilegiada obtém maior taxa de acerto durante o rastreamento visual de exemplos. Os autores afirmam que este é um cenário típico em que a informação privilegiada está acessível apenas durante a etapa de treinamento.

Na Tabela 2.1 é apresentado um resumo dos trabalhos discutidos nesta seção. Para cada trabalho é informado o tipo principal do problema investigado. É possível observar que o paradigma LUPI para agrupamento de dados ainda é pouco explorado na literatura, especialmente para dados textuais. Considerando os desafios existentes e resultados promissores reportados, o paradigma LUPI para cenários não supervisionados é uma direção de pesquisa promissora que é investigada neste trabalho. 
Tabela 2.1: Resumo dos principais trabalhos discutidos que abordagem o paradigma LUPI em problemas de aprendizado de máquina.

\begin{tabular}{|c|c|c|}
\hline Autor(es) & Título & Problema(s) Investigado(s) \\
\hline Vapnik e Vashist (2009) & $\begin{array}{l}\text { A new learning paradigm: Learning using } \\
\text { privileged information }\end{array}$ & $\begin{array}{l}\text { Classificação, Regressão, } \\
\text { Aprendizado de Métricas }\end{array}$ \\
\hline Pechyony e Vapnik (2010) & $\begin{array}{l}\text { On the Theory of Learnining with } \\
\text { Privileged Information }\end{array}$ & $\begin{array}{l}\text { Aspectos teóricos do } \\
\text { classificador SVM+ }\end{array}$ \\
\hline Pechyony e Vapnik (2011) & $\begin{array}{l}\text { Fast Optimization Algorithms for } \\
\text { Solving SVM+ }\end{array}$ & Classificação \\
\hline Cai e Cherkassky (2012) & $\begin{array}{l}\text { Generalized SMO Algorithm for SVM-Based } \\
\text { Multitask Learning }\end{array}$ & Classificação \\
\hline Ribeiro et al. (2010) & Financial distress model prediction using SVM+ & $\begin{array}{l}\text { Classificação, } \\
\text { Análise de Risco }\end{array}$ \\
\hline Ribeiro et al. (2012) & Enhanced default risk models with SVM+ & $\begin{array}{l}\text { Classificação, } \\
\text { Análise de Risco }\end{array}$ \\
\hline Feyereisl e Aickelin (2012) & Privileged information for data clustering & $\begin{array}{l}\text { Agrupamento de Dados, } \\
\text { Seleção de Modelos }\end{array}$ \\
\hline Fouad et al. (2012) & $\begin{array}{l}\text { Learning Using Privileged Information } \\
\text { in Prototype Based Models }\end{array}$ & $\begin{array}{l}\text { Classificação, } \\
\text { Aprendizado de Métricas }\end{array}$ \\
\hline Fouad et al. (2013) & $\begin{array}{l}\text { Incorporating Privileged Information } \\
\text { Through Metric Learning }\end{array}$ & $\begin{array}{l}\text { Classificação, } \\
\text { Aprendizado de Métricas }\end{array}$ \\
\hline Yang e Patras (2013) & $\begin{array}{l}\text { Privileged information-based conditional } \\
\text { regression forest for facial feature detection }\end{array}$ & $\begin{array}{l}\text { Classificação, } \\
\text { Caracteristicas Faciais }\end{array}$ \\
\hline Sharmanska et al. (2013) & Learning to Rank Using Privileged Information & $\begin{array}{l}\text { Classificação de Imagens, } \\
\text { Ranking }\end{array}$ \\
\hline Serra-Toro et al. (2014) & $\begin{array}{l}\text { Exploring some practical issues of SVM+: } \\
\text { Is really privileged information that helps? }\end{array}$ & Classificação \\
\hline Qi et al. (2014) & $\begin{array}{l}\text { A new classification model using privileged } \\
\text { information and its application }\end{array}$ & $\begin{array}{l}\text { Classificação, } \\
\text { Rastreamento de exemplos }\end{array}$ \\
\hline Lapin et al. (2014) & $\begin{array}{l}\text { Learning using privileged information: } \\
\text { SVM+ and weighted SVM }\end{array}$ & Classificação \\
\hline
\end{tabular}

\subsection{Considerações Finais}

Em muitos problemas de aprendizado de máquina há informação adicional a respeito dos dados que pode ser utilizada durante o treinamento do modelo inicial. O paradigma LUPI permite melhorar a tarefa de aprendizado considerando esta informação privilegiada durante a indução dos modelos, tanto de classificação (Vapnik e Vashist, 2009) quanto de agrupamento (Feyereisl e Aickelin, 2012). Estudos recentes indicam que a incorporação da informação privilegiada permite a obtenção de modelos mais robustos, no qual o modelo gerado (pela combinação de informação técnica e privilegida) deve classificar um grande conjunto de exemplos descritos somente pela informação técnica (Pechyony e Vapnik, 2010, 2011).

Em relação às tarefas de agrupamento de dados, o paradigma LUPI é uma alternativa promissora para incorporar informação adicional, valiosa e específica de domínio. Neste paradigma, as múltiplas representação dos dados são utilizadas de maneira mais realista, já que informação adicional de alto nível sobre um determinado problema é naturalmente mais custosa e escassa, ge- 
ralmente limitada para um pequeno subconjunto dos exemplos. Aliado a este desafio, uma organização hierárquica do conhecimento implícito nos textos é desejável, uma vez que este tipo de organização permite a análise exploratória dos dados em diversos níveis de abstração. Combinar as diferentes representações que podem ser extraídas de dados textuais para aprendizado de modelos de agrupamento hierárquico, bem como lidar com diferentes níveis de importância e disponibilidade dessas representações é um tópico de pesquisa em aberto. 
3

\section{Informação Privilegiada em} Agrupamento Hierárquico de Textos

\subsection{Considerações Iniciais}

Estudos sobre extração e organização de conhecimento em dados textuais têm recebido grande atenção nos últimos anos, especialmente após a popularização de inúmeras aplicações web, nas diversas áreas do conhecimento, para publicação e armazenamento de textos (Aggarwal e Zhai, 2012a). Embora dados textuais sejam uma forma natural aos humanos para representação de conhecimento, a falta de estrutura e a presença de ambiguidade os tornam particularmente complexos para serem manipulados de forma automatizada por algoritmos de aprendizado de máquina, sendo esse um ponto crítico para tarefas de extração e organização de conhecimento (Miner et al., 2012). Nesse sentido, a representação estruturada da informação textual é um relevante desafio de pesquisa que tem sido explorado por diversas comunidades, como Aprendizado de Máquina, Recuperação de informação e Mineração de Dados e Textos.

Diferentes modelos para representação estruturada de dados textuais foram propostos na literatura nos últimos anos (Salton et al., 1996; Mladenic e Grobelnik, 1998; Tan et al., 2002; Yang et al., 2003; Carvalho et al., 2010; Aggarwal e Zhai, 2012a). No entanto, o modelo clássico bag-of-words ainda é a principal representação para aplicações de uso geral (Aggarwal e Zhai, 2012a). Neste modelo, os documentos textuais são representados por vetores em um espaço $m$-dimensional, em que cada dimensão corresponde a uma palavra da coleção textual. Em geral, um valor relacionado à frequência de cada palavra é utilizado para indicar a importância das palavras nos documentos. Uma co- 
nhecida limitação do modelo bag-of-words é que a ordem em que as palavras aparecem no texto não é preservada, prejudicando o aspecto semântico desta representação. Por outro lado, existem técnicas para extração de termos compostos (formados por mais de uma palavra), como n-gramas e itemsets, que são alternativas populares para amenizar esta limitação (Aggarwal e Zhao, 2013).

No contexto deste trabalho, o modelo bag-of-words é um exemplo típico de informação técnica, pois possui as duas características principais descritas por Vapnik e Vashist (2009): (1) baixo custo para extração da informação e representação dos dados; e (2) alta disponibilidade tanto na etapa off-line quanto on-line da tarefa de aprendizado. Por outro lado, a diversidade de técnicas para pré-processamento de textos, em especial àquelas que incorporam conhecimento específico de domínio, proporciona diferentes alternativas para representações que possam ser empregadas como informação privilegiada. Considerando os tipos de informação privilegiada descritos por Vapnik e Vashist (2009), neste trabalho focou-se em uma representação textual composta por termos sugeridos/validados por usuários especialistas de domínio. A obtenção desses termos é apoiada por uma estratégia conhecida como seleção interativa de termos, que utiliza aprendizado ativo para incorporar a experiência dos usuários na representação dos textos. As principais contribuições relacionadas à extração e incorporação desta informação privilegiada em tarefas de agrupamento de textos são apresentadas a seguir:

- Uma discussão dos estudos existentes para representação estruturada dos textos é apresentada. Em particular, focou-se em trabalhos que propuseram representações complementares ao modelo bag-of-words, ou seja, que utilizam sequência de palavras (n-gramas e itemsets) como forma para melhorar a compreensão dos termos extraídos. Um segundo aspecto discutido é que a presença de termos específicos de domínio permite um aumento significativo da qualidade de modelos de aprendizado de máquina. Este fato levou ao desenvolvimento de abordagens para apoiar usuários na extração destes termos específicos de domínio. Tais termos são utilizados, nesse trabalho de doutorado, para formar a informação privilegiada do problema;

- Uma ferramenta proposta e desenvolvida neste trabalho, chamada $\mathrm{AL}^{2} \mathrm{FIC}$ (Active Learning to Frequent Itemset-Based Clustering) (Marcacini et al., 2012b), é apresentada como um meio para viabilizar a extração de informa- 
ção privilegiada a partir de coleções textuais. A ferramenta $\mathrm{AL}^{2} \mathrm{FIC}$ utiliza itemsets extraídos dos textos como candidatos a compor informação privilegiada. Em contraste com o uso de termos simples da representação bag-of-words, os itemsets são termos formados por conjuntos de palavras que coocorrem juntas em vários documentos. Por exemplo, considerando uma coleção textual composta por artigos científicos sobre inteligência computacional, os itemsets "\{aprendizado,máquina\}" e "\{agrupamento,dados,multidescrição\}" podem ser selecionados pelo especialista de domínio para formar a representação textual de informação privilegiada. A ferramenta $\mathrm{AL}^{2} \mathrm{FIC}$ realiza a extração dos itemsets dos textos e os organiza em grupos de acordo com a similaridade entre eles. Uma estratégia de aprendizado ativo é aplicada para identificar e apresentar aos usuários uma lista de itemsets candidatos mais representativos de cada grupo. O usuário, por sua vez, seleciona os itemsets que considera mais interessantes de acordo com sua experiência de domínio (seleção interativa de termos), formando-se então um conjunto de termos para compor a informação privilegiada do problema; e

- Por fim é proposta uma abordagem para estender o paradigma LUPI (Learning Using Privileged Information) de Vapnik e Vashist (2009) para cenários não supervisionados, em particular, para agrupamento hierárquico de textos. A abordagem proposta é denominada LIHC (LUPI-Based Incremental Hierarchical Clustering) (Marcacini e Rezende, 2013) e apresenta uma estratégia de consenso de agrupamentos para combinar modelos de agrupamento provenientes da informação técnica e da informação privilegiada. Um cenário experimental foi configurado para avaliar as diferentes variáveis envolvidas nesse processo, como (i) a influência do número de itemsets utilizados para compor a informação privilegiada do problema (número de consultas aos usuários), (ii) a importância de cada representação textual (técnica e privilegiada) durante o aprendizado de um modelo consensual, e (iii) a análise do ganho que a informação privilegiada fornece ao modelo de agrupamento quando comparado com uma abordagem baseada apenas em informação técnica. Os resultados experimentais fornecem evidências de que a informação privilegiada aumenta significativamente a qualidade dos agrupamentos hierárquicos obtidos, indicando que a abordagem proposta neste trabalho é uma alternativa promissora para estender o paradigma LUPI para tarefas de agrupamento. 


\subsection{Representação Estruturada para Dados Textuais}

O modelo clássico bag-of-words para representação estruturada de dados textuais é baseado no modelo espaço-vetorial, no qual cada documento é um vetor em um espaço multidimensional, e cada dimensão é um termo da coleção (Feldman e Sanger, 2006). Conforme comentado anteriormente, na bag-of-words os termos são considerados independentes, formando um conjunto desordenado em que a ordem de ocorrência das palavras não importa. Na Tabela 3.1 é ilustrado um esquema da representação no modelo espaço-vetorial, em que $d_{i}$ corresponde ao i-ésimo documento, $t_{j}$ representa o $j$-ésimo termo e $a_{i j}$ é um valor que relaciona o $i$-ésimo documento com o $j$-ésimo termo. Desta forma, cada documento pode ser representado como um vetor $\vec{d}_{i}=\left(a_{i 1}, a_{i 2}, \ldots, a_{i m}\right)$.

\begin{tabular}{|c|cccc|}
\hline & $t_{1}$ & $t_{2}$ & $\ldots$ & $t_{m}$ \\
\hline \hline$d_{1}$ & $a_{11}$ & $a_{12}$ & $\ldots$ & $a_{1 m}$ \\
$d_{2}$ & $a_{21}$ & $a_{22}$ & $\ldots$ & $a_{2 m}$ \\
$\vdots$ & $\vdots$ & $\vdots$ & $\ddots$ & $\vdots$ \\
$d_{n}$ & $a_{n 1}$ & $a_{n 2}$ & $\ldots$ & $a_{n m}$ \\
\hline
\end{tabular}

Tabela 3.1: Modelo espaço-vetorial para representação de dados textuais

Em relação ao valor da medida $a_{i j}$, em geral, há duas estratégias:

- um valor que indica se um determinado termo está presente ou não em um dado documento; e

- um valor que indica a importância ou distribuição do termo ao longo da coleção de documentos, por exemplo, o valor de TF (Term Frequency). Outras formas, baseadas em critérios de ponderação e normalização, podem ser encontradas em Salton e Buckley (1988) e Lan et al. (2009). Entre elas, destaca-se o critério TF-IDF (Term Frequency Inverse Document Frequency) proposto por Salton et al. (1996), que leva em consideração tanto o valor de TF quanto o valor de DF (Document Frequency).

Para lidar com a limitação da bag-of-words em relação à independência dos termos na representação da coleção textual, diversos estudos investigam o uso de n-gramas e itemsets, que são estratégias para identificar termos compostos visando complementar (e às vezes substituir) o modelo bag-of-words. 
De acordo com Bekkerman e Allan (2004), n-gramas são termos compostos formados por uma sequência de $n$ palavras que ocorrem consecutivamente em um texto. A definição do tamanho máximo de $n$ é uma característica experimental, dependente da coleção textual. A frequência de ocorrência de $n$ gramas nos textos é reduzida conforme o valor de $n$ aumenta, o que pode ser utilizado para aferir o valor máximo de $n$. Em geral, o uso de n-gramas para representação de dados textuais é realizado de duas formas. Na primeira, são utilizados n-gramas com $n>1$, ou seja, considerando apenas termos compostos. Na segunda, utiliza-se $n \geq 1$, o que significa que os termos simples usados na bag-of-words também estão contidos na representação com n-gramas. Uma visão geral dos principais trabalhos e resultados experimentais envolvendo $n$ gramas é apresentada a seguir.

Mladenic e Grobelnik (1998): neste trabalho é realizada uma avaliação experimental do uso de n-gramas como forma de enriquecer a representação bag-of-words, ou seja, com $n \geq 1$. Os autores avaliaram o efeito de diferentes valores de $n$ em tarefas de classificação, com uso do algoritmo Naïve Bayes, usando textos coletados a partir de páginas do Yahoo!. Devido ao grande número de n-gramas extraídos, foi utilizado um subconjunto de termos selecionado por meio da medida Odds Ratio (Forman, 2003). Entre os resultados obtidos, vale destacar que a acurácia de classificação foi melhorada quando $n=2$ e $n=3$, enquanto valores maiores de $n$ não obtiveram melhorias na acurácia de classificação.

Fürnkranz (1998): neste trabalho é apresentado um estudo similar ao de Mladenic e Grobelnik (1998). A principal diferença é que a seleção de $n$ gramas foi realizada por meio das medidas TF (Term Frequency) e DF (Document Frequency). A avaliação das representações foi realizada por meio da respectiva taxa de acerto obtida por regras de classificação geradas pelo algoritmo RIPPER. Nas duas coleções textuais utilizadas, Reuters21578 e 20-Newsgroups, a melhor configuração de n-gramas $(T F=5$, $D F=3$ e $n=3$ ) obteve uma redução de $2,02 \%$ na taxa de erro de classificação quando comparado com as regras obtidas a partir da bag-of-words.

Tan et al. (2002): um estudo comparando termos simples da representação bag-of-words com termos compostos por duas palavras, denominados bigramas (n-gramas com $n=2$ ), é discutido neste trabalho. Nas duas coleções textuais da avaliação experimental, Yahoo! Science e Reuters21578, os autores reportaram que o emprego de bigramas levou a uma 
melhoria estatisticamente significativa na taxa de acerto de classificação, com emprego do algoritmo Nä̈ve Bayes. Ao contrário dos trabalhos anteriores, os autores deste estudo utilizaram uma estratégia mais robusta para selecionar um subconjunto de bigramas, com base na medida Ganho de informação (Forman, 2003). Este estudo indica o quão importante é a tarefa de seleção dos termos para a qualidade da representação dos textos, principalmente em algoritmos que assumem independência entre os atributos, que é o caso do Naïve Bayes.

Carvalho e Cohen (2006): neste trabalho é realizado um estudo sobre uso de $n$-gramas considerando o contexto da aplicação. Os autores discutiram a tarefa de categorizar intenções de e-mails de uma organização, por exemplo, agendar reuniões, realizar um pedido, submeter um relatório e apresentar uma reclamação. A avaliação experimental indicou que o uso de termos compostos dos n-gramas reduz em $26 \%$ a taxa de erro desta categorização, quando comparado com uso de termos simples. Em particular, as menores taxas de erros foram obtidas ao incorporar bigramas $(n=2)$ e trigramas ( $n=3$ ) junto à representação bag-of-words. Neste estudo também foi utilizada a medida Ganho de informação para selecionar um subconjunto de n-gramas, bem como empregado um préprocessamento apropriado para o domínio da aplicação, ou seja, para a coleção de e-mails. Neste caso, durante o pré-processamento foram extraídos termos relacionados aos dias da semana, pronomes de tratamento, expressões numéricas e nomes/extensões dos arquivos anexados. Este trabalho mostra a importância em considerar o contexto do problema (domínio da aplicação) durante a seleção dos termos para a qualidade da representação.

Li et al. (2008): neste trabalho é proposto e avaliado um algoritmo de agrupamento de textos denominado CFWS (Clustering Based on Frequent Word Sequences), que utiliza os n-gramas mais frequentes (aqueles que ocorrem em vários documentos acima de um determinado limiar) como candidatos a grupos. Em cada iteração do algoritmo CFWS, os grupos (ngramas) mais similares são unidos até atingir um determinado critério de parada, por exemplo, o número de grupos desejado. Cada n-grama possui um conjunto de documentos associado, que são os documentos em que o n-grama ocorre. Assim, a similaridade entre os grupos é calculada baseada na intersecção desses conjuntos. Os autores realizaram 
uma avaliação experimental em três bases textuais (Reuters-21578, CISI e HARD-TREC), comparando o algoritmo CFWS com o algoritmo Bisecting $K$-means utilizando a bag-of-words. Os resultados indicaram que o uso de $n$-gramas empregado pelo CFWS obteve um modelo de agrupamento com maior qualidade de acordo com o indice FScore. Outra vantagem apontada pelos autores é que o uso de n-gramas em tarefas de agrupamento facilita a análise exploratória dos resultados, uma vez que os termos compostos mais frequentes de cada grupo podem ser utilizados como descritores do agrupamento.

Carvalho et al. (2010): neste trabalho há uma avaliação do emprego de ngramas no contexto de um sistema de recuperação de informação, especialmente, com uso de bigramas. Os autores propuseram uma abordagem baseada no classificador $S V M$ para identificar bigramas válidos e não válidos em uma coleção de textos. Os bigramas válidos foram utilizados para melhorar o desempenho do sistema de recuperação de informação. Os autores mostraram que, em alguns domínios, a incorporação de usuários especialistas para rotular manualmente os bigramas válidos permitia uma melhora de até $50 \%$ da precisão do sistema de recuperação de informação. Este trabalho mostra a importância em incorporar a informação externa, via especialista de domínio, no processo de seleção dos termos.

Aggarwal e Zhao (2013): em um dos trabalhos mais recentes sobre representação estruturada de dados textuais, os autores apresentaram um modelo denominado DG (Distance Graphs) para representar as relações entre as palavras da coleção textual. O modelo é baseado em um grafo, no qual cada palavra da coleção textual é um nó e uma aresta direcionada é criada para definir a coocorrência entre duas palavras. Assim, se uma palavra $i$ ocorre antes da palavra $j$, então uma aresta é criada com origem em $i$ para o destino $j$. O modelo $D G$ possui um parâmetro de distância máxima $k$ entre as palavras $i$ e $j$ no texto. Neste caso, se duas palavras $i$ e $j$ estão separadas por no mínimo $k$ posições (duas palavras consecutivas possui $k=1$ ), então as palavras $i$ e $j$ são inseridas no grafo, formandose uma aresta. Por fim, cada aresta é ponderada usando a quantidade de vezes que as duas palavras são contabilizadas em toda a coleção textual, considerando a distância máxima $k$. Os autores discutem a relação entre a representação $D G$ com $n$-gramas. No $D G$ a força das relações en- 
tre palavras é computada considerando todos os documentos da coleção (frequência absoluta), enquanto a identificação tradicional de n-gramas é realizada documento por documento (frequência relativa). Outra diferença é que o modelo $D G$ possui apenas a relação entre duas palavras. Cada aresta do grafo (com o seu respectivo peso) é utilizada como um termo composto em um modelo espaço-vetorial, permitindo empregar algoritmos de aprendizado de máquina tradicionais. Os autores avaliaram a representação $D G$ tanto em tarefas de classificação quanto de agrupamento, mostrando resultados promissores em comparação ao uso de termos simples, principalmente $\operatorname{com} k=3$.

Uma alternativa aos n-gramas é o emprego de itemsets para representar termos compostos em coleções textuais. Nesse caso, os termos compostos não são apenas formados por palavras consecutivas em um texto, mas também por conjuntos de palavras que se repetem em vários documentos de uma coleção textual. O uso de itemsets tem sido popular em tarefas de agrupamento de textos, uma vez que o próprio itemset representa um tipo de grupo de documentos, formado pela lista de documentos que contém o determinado itemset. A seguir são discutidos alguns trabalhos que utilizam itemsets em tarefas de agrupamento de textos.

Beil et al. (2002): neste trabalho foi introduzido o uso de itemsets para agrupamento de textos, no qual documentos que compartilham uma quantidade significativa de itemsets são alocados em um mesmo grupo. Os autores propuseram um algoritmo para agrupamento particional, chamado FTC (Frequent Term-Based Clustering), e um algoritmo para agrupamento hierárquico chamado HFTC (Hierarchical Frequent Term-Based Clustering). Uma avaliação experimental em três coleções textuais (Classic3, Reuters e WAP) mostrou que os dois algoritmos não apresentaram resultados superiores, de acordo com o índice $F_{S C O R E}$, comparado ao tradicional Bisecting $k$-means com uso da bag-of-words. No entanto, os autores afirmaram que os resultados são competitivos e apresentam a vantagem de modelos de agrupamento mais compreensiveis aos humanos, uma vez que os itemsets são utilizados como descritores dos grupos.

Fung et al. (2003): um algoritmo alternativo ao FTC e HTFC é apresentado neste trabalho. Os autores propuseram o algoritmo FIHC (Frequent ItemsetBased Hierarchical Clustering) que, de forma análoga aos algoritmos anteriores, obtém agrupamentos baseado na quantidade de itemsets com- 
partilhados por conjuntos de documentos. Os autores discutem que os modelos de agrupamento obtidos pelo FTC e HTFC são sensíveis à escolha dos grupos iniciais. Já no FIHC, após a obtenção do modelo de agrupamento, há uma etapa que faz uma nova alocação de documentos aos grupos de acordo com o grau de compartilhamento de itemsets entre eles. Desta forma, segundo os autores, o efeito de uma má escolha inicial dos grupos pode ser contornado. Em uma avaliação experimental, o algoritmo FIHC obteve resultados significativamente superiores ao FTC e HTFC, de acordo com o índice $F_{S C O R E}$. Porém, também não apresentou melhora significativa quando comparado ao tradicional Bisecting $k$-means com uso da bag-of-words.

Malik e Kender (2006): neste trabalho é apresentado um estudo sobre outras estratégias para extração de itemsets a partir dos textos. Nos trabalhos anteriores, os itemsets eram obtidos considerando a frequência do conjunto de palavras na coleção textual. Em particular, o bem conhecido algoritmo APRIORI (Agarwal e Srikant, 1994) é empregado para extrair os itemsets frequentes, ou seja, àqueles que possuem frequência maior do que um limiar predefinido (parâmetro denominado suporte). Já neste trabalho, os autores propuseram o uso de diversas medidas para avaliação da qualidade de um itemset, que são comumente empregadas na área de extração de regras de associação. Além disso, também há a eliminação de itemsets redundantes, ou seja, itemsets que ocorrem um mesmo conjunto de documentos. Na prática, este trabalho pode ser visto como uma estratégia de seleção de atributos aplicada para identificação de termos compostos mais significativos. Os resultados experimentais reportados indicam que a proposta é competitiva, em relação ao índice $F_{S C O R E}$, quando comparados com algoritmos de agrupamento com base na bag-of-words. No entanto, a escolha da melhor medida para selecionar itemsets se mostrou um problema dependente da coleção textual, o que torna a abordagem difícil de ser aplicada na prática.

Zhang et al. (2010): neste trabalho é apresentado uma avaliação dos métodos anteriores sobre agrupamento baseado em itemsets em dois conjuntos de textos: um chinês e um inglês. Uma nova abordagem, denominada MC (Maximum Capturing), também é proposta visando melhorar o cálculo de similaridade entre documentos com base nos itemsets. Enquanto os trabalhos anteriores definiam que a similaridade entre docu- 
mentos e grupos de documentos era basicamente a quantidade de itemsets compartilhados, os autores deste trabalho apresentam uma medida de similaridade baseada em três componentes: (1) quantidade de itemsets compartilhados; (2) o peso de cada itemset; e (3) medida assimétrica de similaridade. O peso dos itemsets foi obtido de acordo com a frequência relativa dos mesmos na coleção textual. Já o uso de uma medida assimétrica de similaridade foi justificado pelo fato que cada documento possui quantidades diferentes de itemsets. Os resultados experimentais mostraram que o uso da abordagem MC obtém resultados superiores aos algoritmos previamente propostos, de acordo com o índice $F_{S C O R E}$, porém com a ressalva de que não foi realizada uma avaliação com várias coleções textuais nem uma comparação com algoritmos de agrupamento com base na bag-of-words.

Rossi e Rezende (2011): neste trabalho são explorados critérios para seleção de um conjunto de itemsets significativos baseado em medidas de validação comumente utilizadas em regras de associação. As representações obtidas pelos itemsets selecionados por cada medida foram avaliadas em nove coleções textuais, com uso de algoritmos de agrupamento hierárquico e o índice $F_{S C O R E}$ para análise da qualidade do agrupamento. Embora os resultados experimentais reportados indicassem que o uso de itemsets, após a etapa de seleção, leva a uma ligeira melhora da qualidade do agrupamento, não foi possível afirmar que tal melhora é estatisticamente significativa quando comparado com agrupamento a partir da bagof-words. No entanto, uma segunda avaliação foi realizada com usuários especialistas de domínio que avaliaram a qualidade dos descritores dos agrupamentos. Neste caso, os usuários especialistas indicaram que o uso de itemsets melhora de forma significativa a interpretação dos modelos de agrupamento, quando comparado ao uso de termos simples da bag-of-words.

Os trabalhos discutidos até o presente momento apresentam e avaliam representações alternativas ou complementares à bag-of-words. Ao sumarizar os resultados obtidos por esses trabalhos, é possivel estabelecer os cenários em que o uso de n-gramas ou itemsets permite melhorar a qualidade da representação dos textos:

- Quando há uma estratégia robusta para seleção dos termos compostos mais significativos, utilizando-os para complementar a representação 
bag-of-words;

- Quando a compreensibilidade da representação é um requisito importante para a aplicação em questão, por exemplo, ao uso de descritores para modelos de agrupamento; e

- Quando há incorporação de conhecimento externo, como interação com usuários especialistas de domínio, para identificar os termos compostos mais adequados para a representação dos dados textuais.

Em vista disso, uma direção de pesquisa denominada de "Seleção Interativa de Termos" tem reportado resultados promissores relacionados a seleção, compreensibilidade e uso de conhecimento externo para melhorar a representação de dados textuais, conforme descrito na próxima seção.

\subsection{Seleção Interativa de Termos}

Seleção Interativa de Termos é uma forma promissora para incluir a experiência dos usuários em tarefas de agrupamento de textos (Hu et al., 2011; Nourashrafeddin et al., 2013). Ao contrário das estratégias de agrupamento que necessitam de um conjunto inicial de restrições Must-Link e Cannot-Link, a seleção interativa de termos apresenta para os usuários um solução inicial de agrupamento, onde cada grupo possui um conjunto de termos associados (descritores dos grupos). Os usuários podem, então, selecionar os termos mais interessantes de acordo com sua experiência e intuição sobre o domínio do problema. Esta etapa de seleção dos termos é apoiada por técnicas de aprendizado ativo para minimizar o número de interações com o usuário, bem como prover um conjunto adequado de termos candidatos (Raghavan et al., 2005). Após a interação com os usuários, os grupos podem ser refinados, considerando os termos selecionados para aproximar o modelo de agrupamento às expectativas dos usuários.

O primeiro estudo de seleção interativa de termos em dados textuais foi introduzido por Raghavan et al. (2005) para apoiar tarefas de classificação. Os autores utilizaram uma técnica de aprendizado ativo, baseado em critérios de seleção de atributos, para extrair um conjunto de termos para análise dos usuários. A partir da interação com os usuários, a importância dos termos na representação estruturada dos dados textuais é redefinida, ou seja, o peso dos termos selecionados é aumentado em detrimento dos termos não selecionados. Os resultados experimentais, baseados no classificador Naïve 
Bayes, indicaram que humanos possuem boa intuição para identificar termos relevantes, até mesmo quando os usuários não são especialistas do domínio.

No trabalho de $\mathrm{Hu}$ et al. (2011) foi proposto uma abordagem para seleção interativa de termos em tarefas de agrupamento. Nesta abordagem, um modelo de agrupamento inicial é obtido e, para cada grupo, é apresentada uma lista dos termos melhores ranqueados. O ranking é computado com base em uma técnica de aprendizado ativo, geralmente empregando um critério de seleção de atributos. Os usuários podem, então, explorar a lista de termos e indicar quais serão aceitos de acordo com seu conhecimento prévio a respeito do domínio. Os termos selecionados recebem um aumento de seu peso (sua relevância) na representação estruturada da coleção textual. Na última etapa, o algoritmo de agrupamento é executado novamente considerando os novos pesos, obtendo-se assim um modelo de agrupamento mais próximo às expectativas do usuário.

Para realizar uma avaliação experimental mais robusta, Hu et al. (2011) propuseram uma estratégia de simulação de usuários. Neste caso, os usuários simulados têm acesso a uma organização de referência da coleção textual, o que permite calcular o quão adequado um termo é para um determinado grupo. Os resultados experimentais reportados indicam que o uso da seleção interativa de termos permite uma melhora significativa dos modelos de agrupamento, quando comparado com outras técnicas não supervisionadas de seleção de termos. No entanto, os autores ressaltam que o desempenho da abordagem proposta depende da definição empírica de alguns parâmetros, como o número máximo de consultas aos usuários e em até que ponto é interessante aumentar o peso dos termos selecionados na representação.

Mais recentemente, Nourashrafeddin et al. (2013) propuseram uma estratégia de agrupamento de termos, na qual termos correlacionados são alocados em um mesmo grupo. Na primeira etapa do método proposto, os termos mais representativos de cada grupo são apresentados ao usuário, ou seja, há uma etapa de seleção interativa de termos. O conjunto de termos selecionados, chamado de "termos chaves", é empregado para inicializar um novo modelo de agrupamento, em que os documentos são alocados aos termos chaves correspondentes. Os autores discutem que o método proposto obtém modelos de agrupamento superiores aos métodos de seleção de termos tradicionais, sendo uma evidência de que a incorporação do conhecimento dos usuários melhora significativamente a qualidade do agrupamento. Um segundo aspecto discutido no trabalho é que a qualidade da interface (ferramenta de software) de 
interação com os usuários é um ponto crítico da seleção interativa de termos. Neste sentido, os autores exploram o uso de uma nuvem de termos, um mapa bidimensional em que os termos considerados mais relevantes pela técnica de aprendizado ativo são apresentados com maior destaque visual aos usuários.

Apesar das diferenças pontuais entre os estudos apresentados, um aspecto importante que é comum a todos os trabalhos sobre seleção interativa de termos é que é mais intuitivo aos usuários fornecerem "feedback" ao modelo de agrupamento quando há uma interação no nível dos termos, quando comparado ao fornecimento das restrições do tipo Must-Link e Cannot-Link necessários para agrupamento com restrições. Além disso, os termos selecionados representam uma informação rica a respeito do domínio do problema que geralmente são mais eficazes para melhorar a qualidade do agrupamento do que a informação embutida em conjuntos de restrições (Hu et al., 2012). Desta forma, no contexto deste trabalho, o conjunto de termos selecionados pelos usuários é tratado como informação privilegiada do problema.

\subsection{A Ferramenta $\mathrm{AL}^{2}$ FIC para Extração de Informação Privilegiada}

Para viabilizar a extração de informação privilegiada a partir de dados textuais, neste trabalho foi proposta e desenvolvida uma ferramenta para seleção interativa de termos denominada $\mathrm{AL}^{2} \mathrm{FIC}$ (Active Learning to Frequent ItemsetBased Clustering) (Marcacini et al., 2012b). Uma vez que a qualidade e a interpretação dos termos são pontos importantes para a seleção interativa de termos, principalmente pela perspectiva dos usuários, na ferramenta $\mathrm{AL}^{2} \mathrm{FIC}$ são utilizados itemsets durante a seleção interativa de termos. Conforme comentado na Seção 3.2, os itemsets são termos compostos que contribuem no aspecto semântico da representação estruturada dos dados textuais. Uma técnica de aprendizado ativo para minimizar o número de consultas aos usuários é implementada na ferramenta $\mathrm{AL}^{2} \mathrm{FIC}$, com o objetivo de identificar e apresentar apenas os itemsets mais representativos ao usuário durante a etapa da seleção interativa dos termos.

A extração de informação privilegiada por meio da ferramenta $\mathrm{AL}^{2} \mathrm{FIC}$ possui três etapas: (A) agrupamento baseado em itemsets, (B) seleção interativa de termos, e (C) extração de uma nova representação estruturada baseada nos itemsets selecionados. Na Figura 3.1 são ilustrados as três etapas deste processo, descrito em mais detalhes a seguir. 


\begin{tabular}{c|ccccc|} 
& \multicolumn{6}{|c}{ Informação Técnica } \\
\hline \hline Doc 1 & $a_{11}$ & $a_{12}$ & $a_{13}$ & $\ldots$ & $a_{1 \mathrm{~m}}$ \\
Doc 2 & $a_{21}$ & $a_{22}$ & $a_{23}$ & $\ldots$ & $a_{2 m}$ \\
$\vdots$ & $\vdots$ & $\vdots$ & $\vdots$ & $\ddots$ & $\vdots$ \\
Doc n & $a_{n 1}$ & $a_{n 2}$ & $a_{n 3}$ & $\ldots$ & $a_{n m}$ \\
\cline { 2 - 6 }
\end{tabular}

Bag-of-words

\begin{tabular}{c|ccccc|} 
& \multicolumn{6}{|c}{ Informação Privilegiada } \\
\hline \hline Doc 1 & $b_{11}$ & $b_{12}$ & $b_{13}$ & $\ldots$ & $b_{1 r}$ \\
Doc 2 & $b_{21}$ & $b_{22}$ & $b_{23}$ & $\ldots$ & $b_{2 r}$ \\
$\vdots$ & $\vdots$ & $\vdots$ & $\vdots$ & $\ddots$ & $\vdots$ \\
Doc $\mathbf{n}$ & $b_{n 1}$ & $b_{n 2}$ & $b_{n 3}$ & $\ldots$ & $b_{n r}$ \\
\cline { 2 - 5 }
\end{tabular}
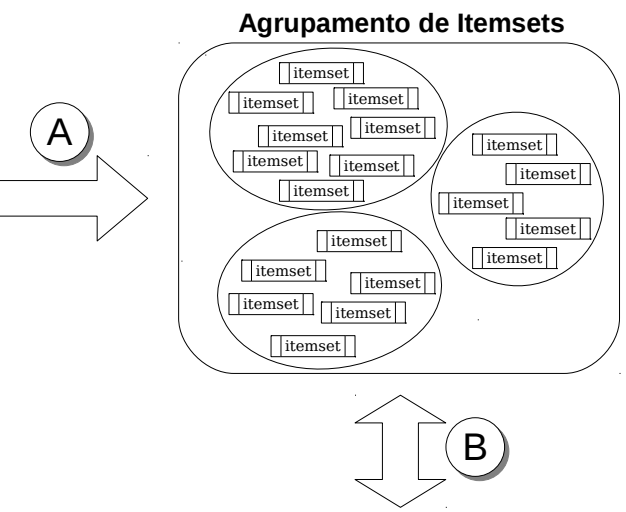

Interação com Usuário

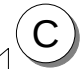

Seleção de Itemsets

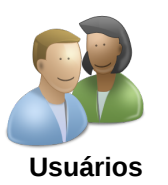

Figura 3.1: Etapas do processo de extração de informação privilegiada por meio da ferramenta AL ${ }^{2}$ FIC: (A) agrupamento baseado em itemsets, (B) seleção interativa de termos, e (C) extração de uma nova representação estruturada baseada nos itemsets selecionados.

Na primeira etapa, uma representação estruturada dos dados textuais baseada na bag-of-words é utilizada como entrada, representando a informação técnica do problema. Relembre que nesta representação, dada uma coleção textual $D$ com $n$ documentos e $m$ termos simples, $D=\left\{d_{1}, d_{2}, \ldots, d_{n}\right\}$, cada documento $i$ é definido como um vetor de termos $\vec{d}_{i}=\left\{t_{1}, t_{2}, \ldots, t_{m}\right\}$, onde $t_{j}$ é um valor que indica a importância do termo $j$ no documento $d_{i}$. Para a extração dos itemsets, esta representação é transformada para um formato binário, ou seja, que indica apenas a ausência ou presença do termo no documento. Na ferramenta $\mathrm{AL}^{2} \mathrm{FIC}$ é utilizada o algoritmo Apriori (Agarwal e Srikant, 1994) para extrair um conjunto de itemsets $F=\left\{f_{1}, f_{2}, \ldots, f_{r}\right\}$. No contexto deste trabalho, cada itemset é definido como uma tripla $f_{s}=\left(W_{s}, T_{s}, \overrightarrow{f_{s}}\right)$, em que $W_{s}$ é o termo composto que identifica o itemset, $T_{s}$ é um conjunto de documentos em que o itemset ocorre, e

$$
\overrightarrow{f_{s}}=\frac{1}{\left|T_{s}\right|} \sum_{\forall \vec{d} \in T_{s}} \vec{d}
$$

é um vetor médio que sumariza o itemset considerando todos os termos simples - de informação técnica - que estão presentes nos documentos do conjunto $T_{s}$. 
O agrupamento dos itemsets é baseado no conjunto de vetores $\left\{\vec{f}_{1}, \vec{f}_{2}, \ldots, \vec{f}_{r}\right\}$. Observe que, com esta representação no modelo espaço-vetorial, algoritmos tradicionais de agrupamento de textos podem ser empregados. No caso da ferramenta $\mathrm{AL}^{2} \mathrm{FIC}$, é empregado o algoritmo de agrupamento particional kmeans, em que $k$ (número de grupos) irá representar o número máximo de interações/consultas com o usuário.

Na segunda etapa do processo, a seleção interativa de termos recebe como entrada uma partição $P=\left\{C_{1}, C_{2}, \ldots, C_{k}\right\}$ e é definido um ranking considerando os itemsets mais relevantes de cada grupo. Para calcular esta relevância, foi proposto o critério definido na Equação 3.2:

$$
\operatorname{score}\left(f_{s}, C_{l}\right)=\left|T_{s}\right| \times\left(\frac{\overrightarrow{f_{s}} \cdot \overrightarrow{c_{l}}}{\left\|\overrightarrow{f_{s}}\right\|\left\|\overrightarrow{c_{l}}\right\|}\right)
$$

Neste caso, a importância de um itemset é baseada no número de documentos em que o itemset ocorre $\left(\left|T_{s}\right|\right)$, e na proximidade do itemset ao centroide $\vec{c}_{l}$ do grupo $C_{l}$ em que está alocado. Em resumo, se um itemset $f_{s}$ possui alta cobertura no conjunto de dados e está alocado de forma apropriada em seu grupo (próximo ao centro do grupo), então $f_{s}$ irá obter uma pontuação alta e boa posição no ranking. Os usuários, então, selecionam os itemsets mais apropriados de cada grupo considerando sua experiência no domínio do problema e com apoio do ranking obtido.

Finalmente, na última etapa os itemsets selecionados são utilizados para extrair uma nova representação estruturada dos textos, ou seja, a informação privilegiada do problema. Neste caso, cada itemset $f_{s}$ possui um peso que define sua importância em relação a um determinado documento $d_{i}$, computado por meio da correlação cosseno definida na Equação 3.3:

$$
\operatorname{peso}\left(d_{i}, f_{s}\right)=\frac{\overrightarrow{f_{s}} \cdot \overrightarrow{d_{i}}}{\left\|\overrightarrow{f_{s}}\right\|\left\|\vec{d}_{i}\right\|}
$$

Quanto mais próximo de 1 for o peso $\left(d_{i}, f_{s}\right)$, maior a importância do itemset ao documento. Caso contrário, o valor do peso se aproxima de 0 .

Na Figura 3.2 é ilustrada a interface da ferramenta $\mathrm{AL}^{2} \mathrm{FIC}$ em uma coleção textual composta por artigos sobre recuperação de informação, visualização de dados, algoritmos paralelos, redes de computadores e acessibilidade web. No Passo 1 é apresentada a lista de itemsets com melhores posições no ranking de um determinado grupo para análise dos usuários. No Passo 2 é apresentado um exemplo da nova representação textual baseada nos itemsets selecionados, ou seja, a informação privilegiada do problema. Neste exemplo, 
foram selecionados cinco termos compostos para a representação de informação privilegiada: "sensor network", "volume image", "document retrieval", "web development", e "queries search". Tais termos representam os temas existentes na coleção textual explorada, ou seja, são termos específicos do domínio.

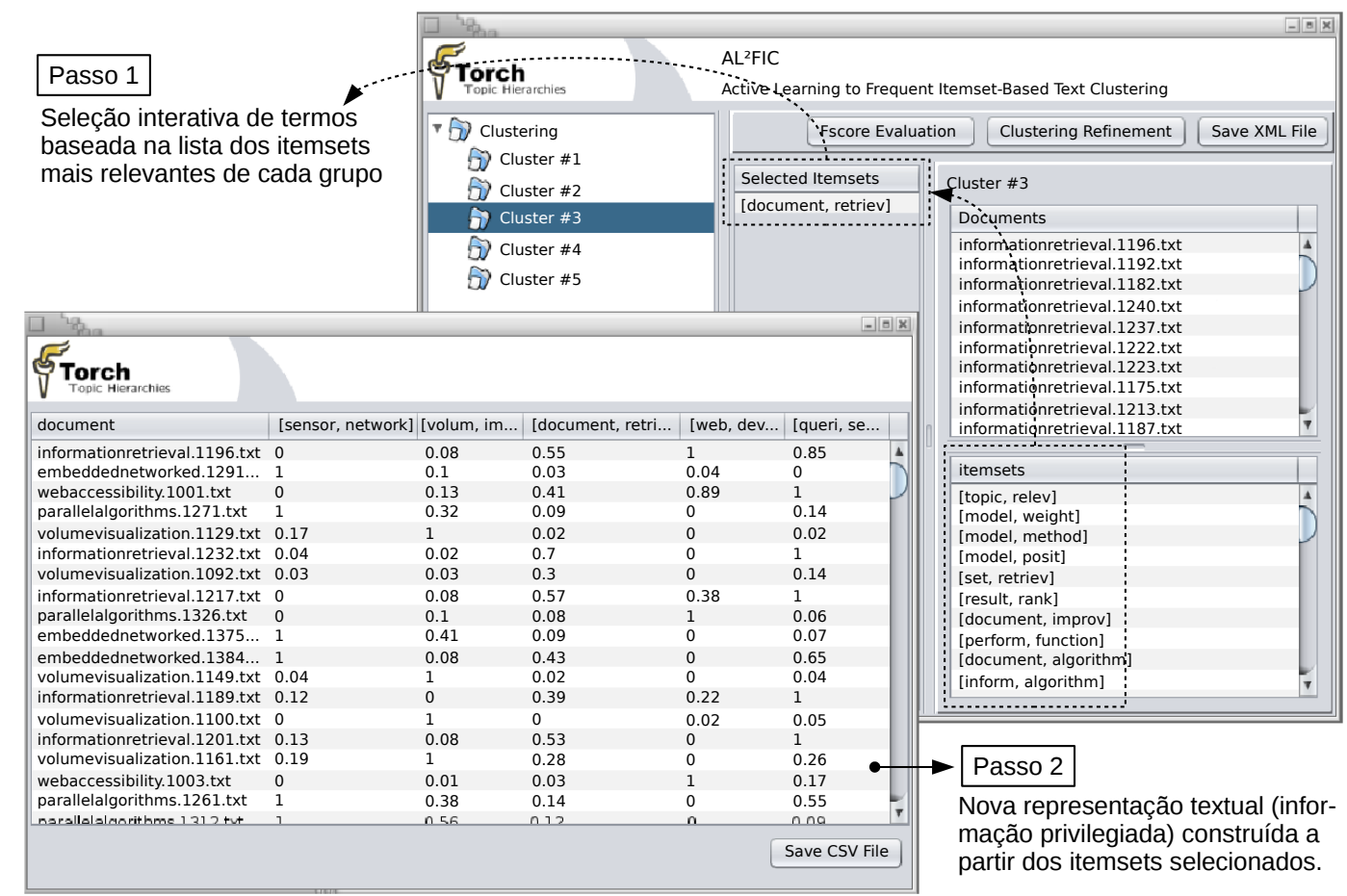

Figura 3.2: Visão geral da interface da ferramenta $\mathrm{AL}^{2} \mathrm{FIC}$ utilizada para extrair informação privilegiada em coleções textuais.

A representação textual de informação privilegiada obtida pela ferramenta $\mathrm{AL}^{2} \mathrm{FIC}$ pode ser empregada em diversas tarefas relacionadas à extração e organização do conhecimento. É por meio do desempenho dessas tarefas que é possível aferir a real eficácia da seleção interativa dos termos. Como o objetivo deste trabalho é investigar o paradigma LUPI (Learning Using Privileged Information) em cenários não supervisionados, a informação privilegiada extraída pela ferramenta $\mathrm{AL}^{2} \mathrm{FIC}$ é incorporada em um modelo de agrupamento, conforme discutido a seguir.

\subsection{Abordagem Proposta: LIHC}

Nesta seção é apresentada a abordagem LIHC (LUPI-Based Incremental Hierarchical Clustering) (Marcacini et al., 2013; Marcacini e Rezende, 2013), proposta para incorporar informação privilegiada em agrupamento hierárquico de textos. A abordagem LIHC permite utilizar as duas representações textuais 
do problema, informação técnica e informação privilegiada, durante o aprendizado de um modelo de agrupamento inicial. Os documentos que não estão representados na informação privilegiada, ou novos documentos apresentados ao modelo, são inseridos no agrupamento por meio de uma estratégia de agrupamento incremental.

Um dos pontos chaves da abordagem LIHC é o uso de consenso de agrupamentos para aprendizado do modelo inicial. Como discutido em Jain (2010), cada algoritmo de agrupamento possui um viés particular em relação ao modelo de agrupamento que será induzido, que geralmente é limitado. O uso de consenso de agrupamentos é uma estratégia útil para contornar este problema, permitindo combinar diferentes soluções de agrupamento e representações dos textos em uma solução única e potencialmente mais robusta de agrupamento (Jain, 2010).

\subsubsection{Estratégia para Consenso de Agrupamentos}

$\mathrm{Na}$ abordagem LIHC é proposto a seguinte estratégia para consenso de agrupamentos:

1. Considere o conjunto com $m$ documentos

$$
X=\left\{\left(\overrightarrow{x_{1}}, \overrightarrow{x_{1}^{*}}\right), \ldots,\left(\overrightarrow{x_{m}}, \overrightarrow{x_{m}^{*}}\right)\right\}, \overrightarrow{x_{i}} \in \chi, \overrightarrow{x_{i}^{*}} \in \chi^{*},
$$

e que $|\chi|$ e $\left|\chi^{*}\right|$ representam, respectivamente, a quantidade de termos nas representações textuais de informação técnica $\chi$ e informação privilegiada $\chi^{*}$. Em seguida, são extraídas diversas partições de dados, por meio da execução de vários algoritmos de agrupamento, tanto no espaço de informação técnica quanto no espaço de informação privilegiada;

2. As partições provenientes de cada representação são sumarizadas por meio de uma matriz de coassociação, cujos elementos são definidos como $\frac{a_{i j}}{p}$, em que $a_{i j}$ representa o número de vezes que os documentos $i$ e $j$ são alocados juntos em um mesmo grupo, considerando as $p$ partições de uma determinada representação (informação técnica ou informação privilegiada);

3. Assim, são obtidas duas matrizes de coassociação: $\mathbf{M}_{t e c}$ obtida a partir da representação textual de informação técnica (tradicional bag-of-words) e $\mathbf{M}_{p r i}$ obtida a partir da representação textual de informação privilegiada (obtida, por exemplo, por meio da ferramenta $\mathrm{AL}^{2} \mathrm{FIC}$ ); 
4. A matriz de coassociação final $\mathbf{M}_{F}$, que combina a informação técnica e informação privilegiada, é definida conforme a Equação 3.4:

$$
\mathbf{M}_{F}=\alpha \mathbf{M}_{t e c}+(1-\alpha) \mathbf{M}_{p r i}
$$

O parâmetro $\alpha(0 \leq \alpha \leq 1)$ indica o Fator de Contribuição (importância) de cada representação para o consenso de agrupamentos; e

5. A matriz $\mathbf{M}_{F}$ representa uma nova relação de similaridade entre os documentos. Assim, para obter o agrupamento hierárquico consensual, a abordagem LIHC utiliza o algoritmo de agrupamento aglomerativo UPGMA a partir de $\mathbf{M}_{F}$. O agrupamento resultante representa o aprendizado do modelo inicial do paradigma LUPI para o cenário não supervisionado.

Após o aprendizado do modelo inicial, com a combinação da informação técnica e informação privilegiada por meio de um agrupamento consensual, os documentos que não pertencem à representação textual de informação privilegiada, ou novos documentos da coleção, são apresentados e inseridos ao modelo. A inserção é realizada de maneira incremental, utilizando uma estratégia de agrupamento incremental top-down (da raiz para as folhas). Um novo documento é inserido no grupo mais similar, por exemplo, por meio da similaridade entre documento e centroide do grupo. O processo avança até que o documento encontre um grupo folha no agrupamento hierárquico (Marcacini et al., 2012c).

\subsubsection{Aspectos Teóricos}

A abordagem LIHC (LUPI-Based Incremental Hierarchical Clustering) é apresentada como uma extensão do paradigma de aprendizado de máquina baseada em informação privilegiada para cenários não supervisionados, em particular, para tarefas de agrupamento de textos. Para tal, assume-se que a informação privilegiada proveniente da seleção interativa de termos da ferramenta $\mathrm{AL}^{2} \mathrm{FIC}$ está disponível apenas durante o aprendizado do modelo inicial. Esta é a situação mais comum em tarefas de agrupamento, uma vez que a consulta aos usuários geralmente é realizada em um cenário off-line em uma amostra representativa de textos do domínio do problema. O modelo inicial é utilizado na etapa on-line para agrupar o restante de documentos de informação técnica. Como todo processo de aprendizado incremental, com o passar do tempo o modelo inicial poderá sofrer uma degradação, uma vez que novos 
conhecimentos podem surgir. Também é importante observar que, na presença de nova informação privilegiada, o aprendizado do modelo inicial pode ser repetido.

Em relação à complexidade da abordagem $\mathrm{LIHC}$, é importante lembrar que algoritmos de agrupamento hierárquico usualmente possuem complexidade quadrática de tempo. No entanto, o aprendizado do modelo inicial geralmente é aplicada em uma amostra de textos que estão representadas em ambas as representações de informação técnica e informação privilegiada. Em situação práticas, é importante que tanto a extração da informação privilegiada quanto o aprendizado do modelo inicial seja realizada a partir de uma amostra representativa do domínio. Para tal, técnicas para identificar um conjunto de documentos representativos podem ser empregadas (Bonin et al., 2014). Assim, dada uma amostra de tamanho $m$ de uma coleção com $n$ documentos, a complexidade total da abordagem LIHC é $O_{i n i}(s)^{2}+O_{i n c}(n k)$. Neste caso, $O_{i n i}(s)^{2}$ é a complexidade para aprendizado do modelo inicial, e $O_{i n c}(n k)$ é a complexidade para a etapa de agrupamento incremental, em que $k$ representa o número de grupos e subgrupos do modelo inicial. Considerando as variáveis dominantes do problema, e assumindo que $m<<n$, a complexidade de tempo é linear com respeito tanto a $n$ e $k$.

\subsection{Análise Experimental}

Para verificar a eficácia da abordagem LIHC em incorporar informação privilegiada em aprendizado não supervisionado, foi realizada uma avaliação experimental com o objetivo de elucidar as seguintes questões:

1. Gual a influência da quantidade de itemsets selecionados (número de consultas aos usuários pela ferramenta $\mathrm{AL}^{2} \mathrm{FIC}$ durante a extração de informação privilegiada) em relação à acurácia do modelo de agrupamento inicial?

2. Qual a influência do parâmetro Fator de Contribuição $(\alpha)$ durante o consenso de agrupamentos? Em outras palavras, qual a importância de cada representação (informação técnica e informação privilegiada) durante o aprendizado do modelo inicial?

3. Qual o ganho obtido na acurácia do modelo de agrupamento final (após a inserção incremental de todos os documentos) da abordagem LIHC, 
quando comparada com uma estratégia baseada apenas em informação técnica?

Para responder as questões 1 e 2, foi realizada uma análise de parâmetros da abordagem LIHC em seis coleções textuais retiradas da Biblioteca Digital da $\mathrm{ACM}^{1}$. As coleções são compostas por artigos de Ciência de Computação, uma temática que facilita (para o autor da tese) analisar subjetivamente a qualidade dos itemsets extraídos. Além disso, a Biblioteca Digital da ACM possui categorias de referência que permitem avaliar objetivamente a qualidade dos modelos de agrupamento. Para responder a questão 3, foi realizada uma avaliação experimental comparando a abordagem LIHC com uma abordagem tradicional de consenso de agrupamentos utilizando apenas informação técnica (bag-of-words). Tal avaliação foi realizada em dez coleções textuais de benchmark frequentemente utilizadas em tarefas de agrupamento de textos. Os detalhes das coleções textuais, configuração experimental e discussão dos resultados são apresentados nas próximas seções.

\subsubsection{Configuração dos Experimentos}

O consenso de agrupamentos utilizado na abordagem LIHC possui o parâmetro $\alpha$ denominado Fator de Contribuição, que determina a importância de cada representação textual durante o consenso de agrupamentos. Foram analisados 11 possiveis valores para o parâmetro $\alpha$ : 0,0.1, 0.2, 0.3, 0.4, 0.5, 0.6, 0.7, $0.8,0.9$ e 1 . Quando $\alpha=0$ significa que o consenso de agrupamentos está utilizando apenas a informação técnica do problema. Por outro lado, quando $\alpha=1$ significa que o consenso de agrupamentos está utilizando apenas a informação privilegiada. Quando $0<\alpha<1$, então as duas representações textuais são utilizadas, cada uma com determinado nível de importância.

Para realizar o consenso de agrupamentos é necessário obter diversas partições dos dados de ambas as representações. Neste trabalho foram utilizadas diversas execuções do algoritmo k-means, com diferentes inicializações e números de grupos $(k)$. Em particular, os valores de $k$ foram selecionados aleatoriamente entre $\{2,3, \ldots, \sqrt{m}\}$, em que $m$ é o número de documentos utilizados para aprendizado do modelo inicial. A medida de similaridade cosseno foi utilizada no algoritmo k-means. Cada matriz de coassociação é computada a partir de 100 partições. A avaliação da qualidade do modelo de agru-

\footnotetext{
${ }^{1}$ ACM Digital Library: http://dl .acm.org/
} 
pamento hierárquico é realizada com uso da medida $F_{S C O R E}$ (ver Capítulo 2, Seção 2.2.3).

Por fim, para analisar o efeito da quantidade de itemsets selecionados em relação à qualidade do modelo inicial, foram avaliados os seguintes números de consultas aos usuários: 5, 10, 25, 50, 75, 100, 150, 200, 300, 400 e 500. Devido ao grande número de variáveis envolvendo a configuração experimental, bem como a necessidade de executar repetidamente os experimentos para analisar a estabilidade das soluções obtidas, foi adotada uma técnica para simular interações do usuário durante a seleção interativa de termos, conforme apresentado a seguir.

\subsubsection{Simulando Interações com o Usuário}

O processo de simulação é baseado no conhecimento prévio a respeito das categorias dos documentos, seguindo a estratégia apresentada em outros estudos sobre seleção interativa de termos (Hu et al., 2011; Nourashrafeddin et al., 2013). Neste caso, quando a lista de itemsets é apresentada para cada grupo, o processo de simulação irá escolher o itemset que apresenta menor entropia de acordo com a Equação 3.5.

$$
\operatorname{entropy}\left(f_{s}\right)=\sum_{z=1}^{c}-\frac{\left|L_{z} \cap T_{s}\right|}{\left|T_{s}\right|} \log \left(\frac{\left|L_{z} \cap T_{s}\right|}{\left|T_{s}\right|}\right)
$$

Nesta equação, $T_{s}$ representa o conjunto de documentos cobertos pelo itemset $f_{s}, L_{z}$ é o conjunto de documentos que pertence a uma determinada categoria $z$ do conjunto de documentos, e $c$ é o número total de categorias. Um valor baixo de entropia indica que o itemset representa bem uma determinada categoria.

É importante observar que o processo de simulação dos usuários não é livre de erros, sendo que mesmo em um processo simulado alguns itemsets selecionados podem representar ruído ou serem irrelevantes. Note que o ranking de itemsets é obtido de forma não supervisionada considerando apenas a estrutura do agrupamento de itemsets (não há consulta às categorias de referência). Além disso, o próprio algoritmo de agrupamento utilizado para organizar os itemsets em $k$ grupos é suscetível a obter soluções fracas (ótimos locais).

\subsubsection{Análise de Parâmetros}

A avaliação experimental para análise dos parâmetros, como o número de itemsets selecionados e o Fator de Contribuição (parâmetro $\alpha$ ), é realizada 
considerando seis coleções textuais compostas por artigos da área da Ciência da Computação. Os artigos foram coletados a partir da Biblioteca Digital da $\mathrm{ACM}^{2}$. Na Tabela 3.2 é apresentado um sumário dessas coleções textuais. Cada coleção textual é organizada em cinco categorias de referência. Os documentos foram pré-processados com uso da ferramenta JPretext ${ }^{3}$. Foram removidos as stopwords e o algoritmo de Porter foi aplicado para radicalização dos termos. Além disso, os termos que ocorrem em menos de dois documentos foram removidos. A saída desta etapa de pré-processamento é uma representação textual de informação técnica (bag-of-words) com uso da medida TFIDF para ponderação dos termos.

Tabela 3.2: Detalhes das Coleções Textuais usadas na Análise de Parâmetros.

\begin{tabular}{l|c|c}
\hline \hline Temas da Coleções Textuais & \#Documentos & \#Termos \\
\hline \hline Databases Systems and Simulations & 469 & 1964 \\
\hline Eletronic Society and Data Management & 416 & 1620 \\
\hline Information Retrieval and Networking & 394 & 1608 \\
\hline Software and Data Visualization & 399 & 1854 \\
\hline Theory of Computing and Education & 471 & 1977 \\
\hline Web and Data Mining & 497 & 1953 \\
\hline \hline
\end{tabular}

Na Figura 3.3 é apresentada uma análise geral dos parâmetros $\alpha$ (Fator de Contribuição) e do número de itemsets selecionados, nas seis coleções textuais. Nestes resultados, observa-se que quanto maior o valor da medida $F_{S C O R E}$, mais escura é a cor da célula. Dessa forma, é possível comparar visualmente a acurácia do modelo de agrupamento conforme a variação dos dois parâmetros. O teste estatístico de Wilcoxon foi aplicado para comparar se determinada configuração obtém resultados superiores à configuração de referência. Nesse caso, a configuração de referência é o consenso de agrupamento sem uso de informação privilegiada $(\alpha=0)$. Valores sublinhados indicam as configurações que obtiveram resultados superiores à de referência, com $95 \%$ de confiança.

Os resultados experimentais desta análise dos parâmetros fornecem evidências de que a incorporação de informação privilegiada permite aumentar significativamente a qualidade do modelo de agrupamento inicial. Em particular, os valores da medida $F_{S C O R E}$ aumentam em praticamente todos os cenários quando o Fator de Contribuição $(\alpha)$ está definido no intervalo [0.4, 0.7], sugerindo a escolha de valor balanceado para definir a importância da

\footnotetext{
${ }^{2}$ Biblioteca Digital da ACM: http://dl.acm.org

33Pretext: http://sites.labic.icmc.usp.br/torch/msd2011/jpretext/
} 


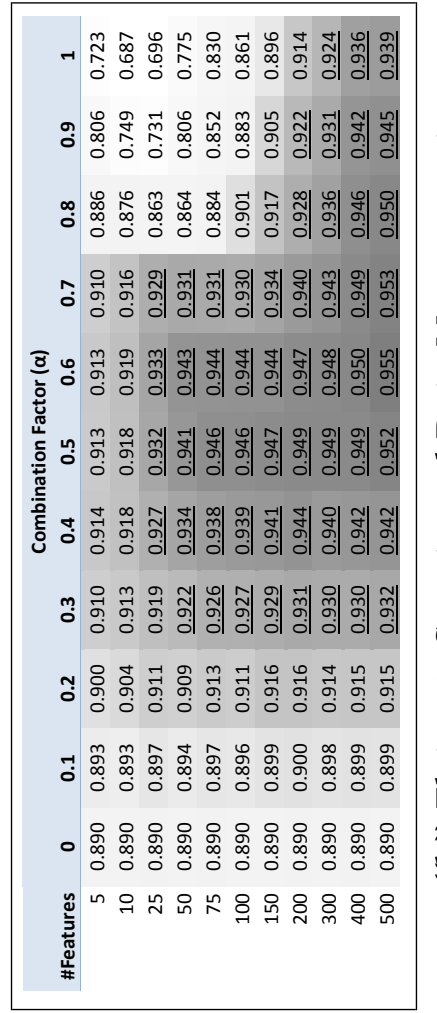

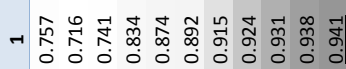

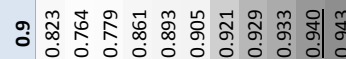

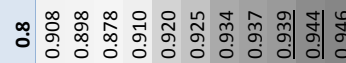

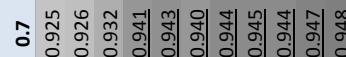

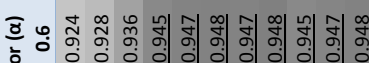

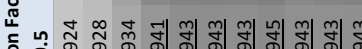
音次

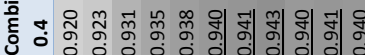

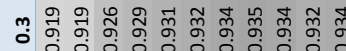

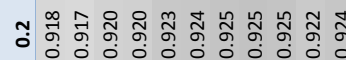

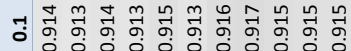

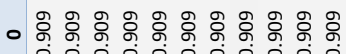

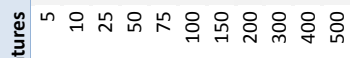

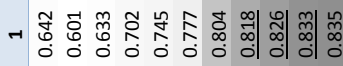

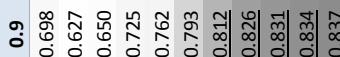

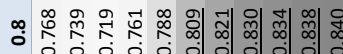
:

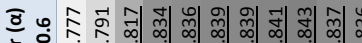

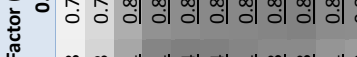

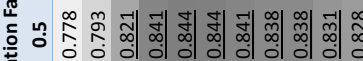

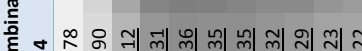

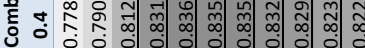

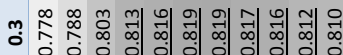

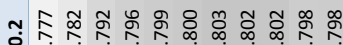

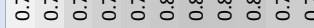

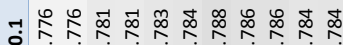
00000000000 -

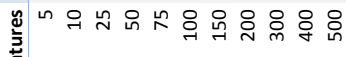
要

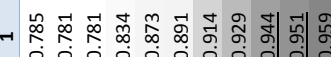

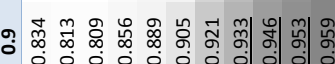

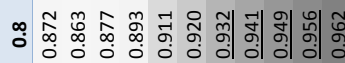

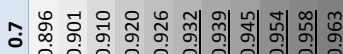

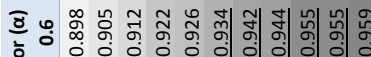

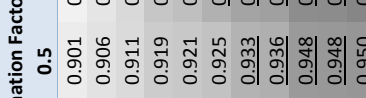

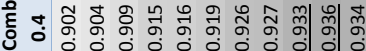

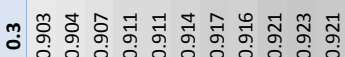

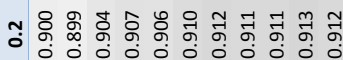

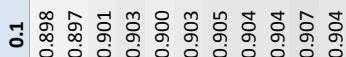

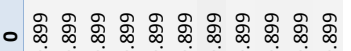

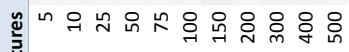

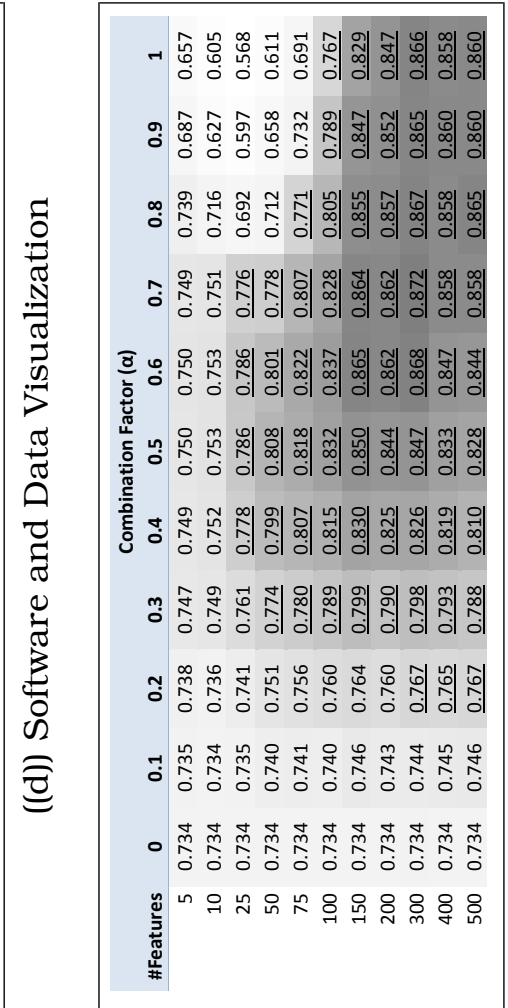

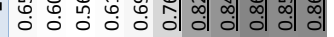

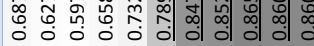

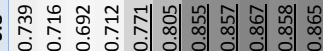

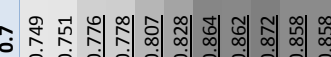

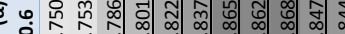

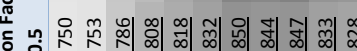

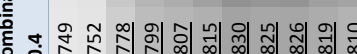
추의

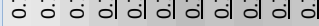

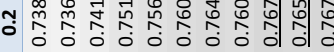

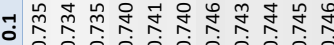

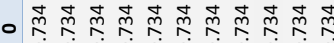

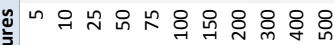
\#

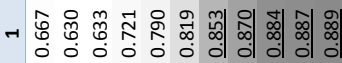

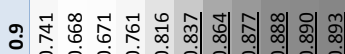

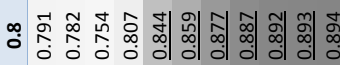

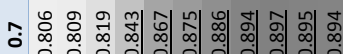
శซㅡㄴ 意

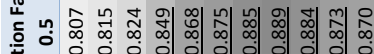

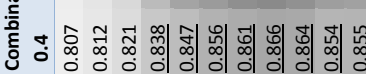

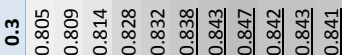

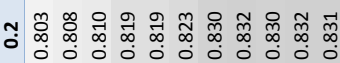

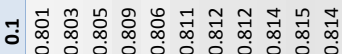

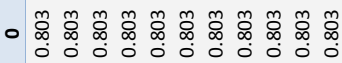

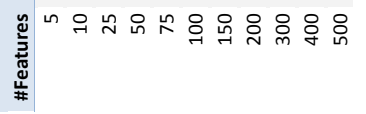



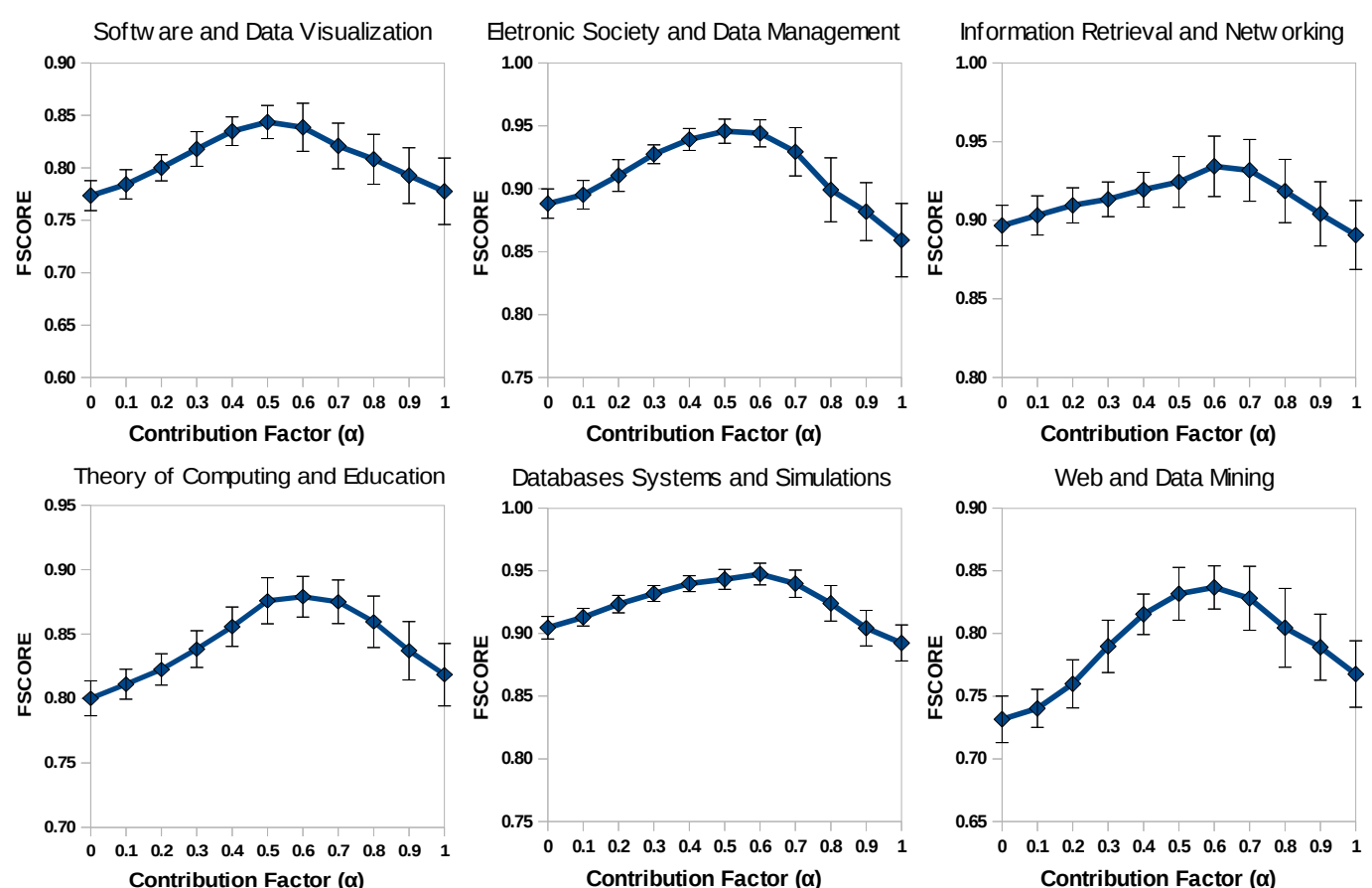

Figura 3.4: Valores $F_{S C O R E}$ para consenso de agrupamentos considerando 100 itemsets na representação textual de informação privilegiada.

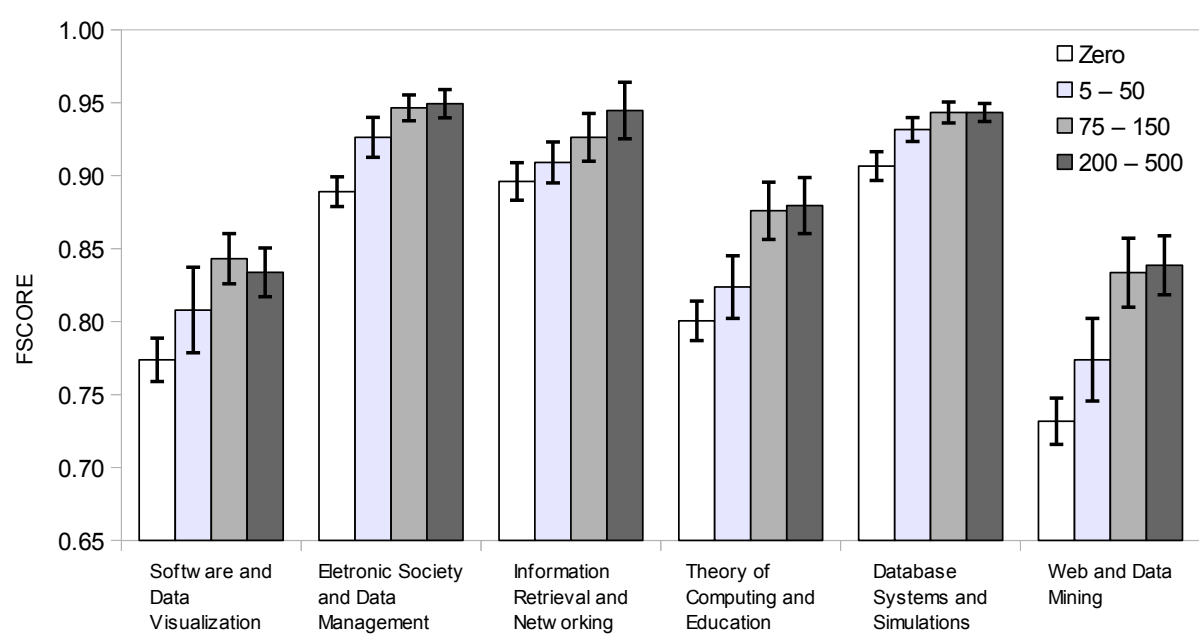

Figura 3.5: Valores $F_{S C O R E}$ considerando diferentes níveis de consulta aos usuários para cada coleção textual.

informação técnica e informação privilegiada durante o consenso de agrupamentos. A Figura 3.4 ilustra melhor esse aspecto (a quantidade de itemsets selecionado foi fixada em 100).

Finalmente, na Figura 3.5 é apresentada uma comparação dos valores $F_{S C O R E}$ em relação a quatro diferentes níveis de consultas ao usuário: zero (em que não há interação seletiva de termos), 5-50, $75-150$ e $200-500$. 
Para esta análise, foi utilizado um valor balanceado para o Fator de Contribuição, ou seja, $\alpha=0.5$. Uma análise estatística desses resultados revela que $(200-500,75-150)>5-50>$ Zero, em que a ordem dos níveis de consulta aos usuários reflete a posição no rank e "> indica diferença estatisticamente significativa. Em todos os casos, a incorporação de informação privilegiada aumenta a qualidade do modelo inicial, mesmo quando poucos itemsets são obtidos pela seleção interativa de termos.

\subsubsection{Agrupamento Hierárquico Incremental}

Na seção anterior foi realizada uma análise dos parâmetros da abordagem LIHC. Nesta seção, é apresentada uma avaliação experimental de todo o processo de agrupamento, desde o aprendizado do modelo inicial (etapa off-line) e o agrupamento hierárquico incremental (etapa on-line).

Considerando os resultados experimentais da análise de parâmetros do LIHC, a incorporação da informação privilegiada se mostrou efetiva durante o aprendizado do modelo de agrupamento inicial, o que corresponde à etapa off-line do paradigma de aprendizado LUPI (Learning Using Privileged Information). Conforme estabelecido no paradigma LUPI, a fase on-line é responsável para que o restante dos documentos (representados apenas pela informação técnica) sejam incorporados ao modelo inicial. No contexto deste trabalho, a etapa on-line é apoiada por uma estratégia de agrupamento incremental, comparando-se a similaridade cosseno entre novos documentos e os centroides dos grupos e subgrupos existentes na hierarquia.

Com base nos resultados da análise de parâmetros, foi definida a seguinte configuração para esta avaliação experimental: $\alpha=0.5$ que define um Fator de Contribuição balanceado em relação à importância das representações de informação técnica e informação privilegiada; e número máximo de itemsets igual a 150, que define a quantidade de termos selecionados para constituir a informação privilegiada.

A avaliação experimental foi realizada com base em 10 coleções textuais de benchmark comumente utilizadas na análise de agrupamento de textos. Os textos foram pré-processados por meio da ferramenta JPretext, com remoção de stopwords e uso do algoritmo de Porter para radicalização dos termos. A ferramental $\mathrm{AL}^{2} \mathrm{FIC}$ foi empregada para a seleção interativa de termos com uso de usuários simulados. Na Tabela 3.3 é apresentada uma descrição geral das coleções textuais, incluindo o número de documentos e termos da representação de informação técnica (bag-of-words), o número de documentos e termos 
da representação de informação privilegida, e o número de categorias de referência. Informações mais detalhadas a respeito destas coleções textuais foram disponibilizadas no relatório técnico de Rossi et al. (2013a).

Tabela 3.3: Detalhes das coleções textuais utilizadas na avaliação experimental

\begin{tabular}{r|c|c|c|c|c}
\hline \hline & \multicolumn{2}{|c|}{ Informação Técnica } & \multicolumn{2}{c}{ Informação Privilegiada } & \\
\hline \hline Coleção Textual & \#Documentos & \#Termos & \#Documentos & \#Termos & Categorias \\
\hline 2Ong & 18828 & 18146 & 2421 & 150 & 20 \\
\hline ACM & 3498 & 3034 & 783 & 149 & 40 \\
\hline Hitech & 2301 & 3742 & 340 & 142 & 6 \\
\hline LATimes & 6279 & 5911 & 797 & 149 & 6 \\
\hline NSF & 10524 & 3861 & 1421 & 150 & 16 \\
\hline Ohsumed4OO & 9200 & 8300 & 1351 & 150 & 23 \\
\hline PubMed-Cancer & 15588 & 7805 & 1931 & 150 & 12 \\
\hline Re8 & 7674 & 5235 & 984 & 140 & 8 \\
\hline Reviews & 4069 & 5628 & 533 & 128 & 5 \\
\hline \hline WebACE & 3900 & 5895 & 683 & 135 & 21 \\
\hline
\end{tabular}

O subconjunto de documentos que estão representados tanto pela informação técnica quanto pela informação privilegiada, aqui chamado de $S_{0}$, é empregado para aprendizado do modelo de agrupamento hierárquico inicial. Para analisar o processo de agrupamento incremental, o restante dos documentos foram divididos em dez subconjuntos: $S_{1}, S_{2}, S_{3}, S_{4}, S_{5}, S_{6}, S_{7}, S_{8}, S_{9}$ e $S_{10}$.

Durante o agrupamento de cada subconjunto de documentos, o índice $F_{S C O R E}$ é computado para aferir a acurácia do modelo em vários estágios do processo. A abordagem LIHC (LUPI-Based Incremental Hierarchical Clustering) é comparada com uma estratégia tradicional de consenso de agrupamentos sem uso de informação privilegiada, aqui denominada CC (Consensus Clustering). Dessa forma, é possível verificar qual o ganho efetivo na incorporação da informação privilegiada durante o agrupamento do LIHC.

Nos gráficos da Figura 3.6 é possível notar que a escolha do modelo inicial é importante para todo o processo de agrupamento incremental. Na maioria dos casos, a abordagem que seleciona o melhor modelo inicial $\left(S_{0}\right)$ também obtém a melhor acurácia na solução final de agrupamento $\left(S_{10}\right)$. Este comportamento está ilustrado de forma mais clara nos resultados obtidos em seis coleções textuais 20ng, Hitech, PubMed-Cancer, Re8, Reviews e WebACE, em que a informação privilegiada foi incorporada de forma mais efetiva. Já nas coleções textuais $A C M$, LATimes e $N S F$, embora nota-se um aumento na acurácia do modelo com a incorporação de informação privilegiada, tal melhora é menos significativa. Por fim, na coleção textual Ohsumed400 é ilustrado o caso em que os modelo iniciais obtidos pelas duas abordagens apresentam qualidade 


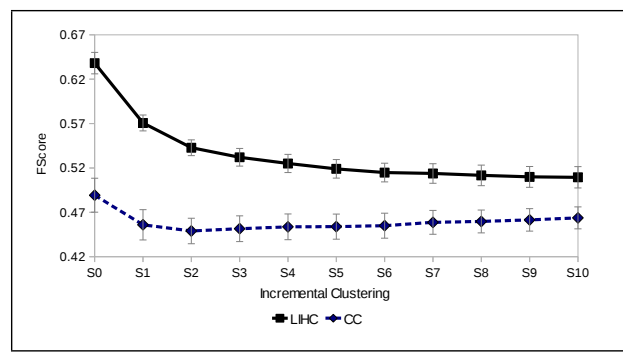

((a)) 20ng

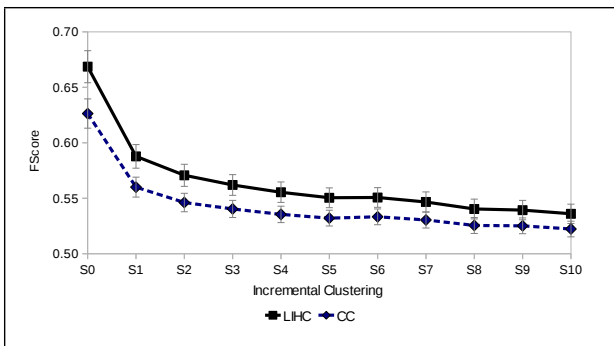

((c)) Hitech

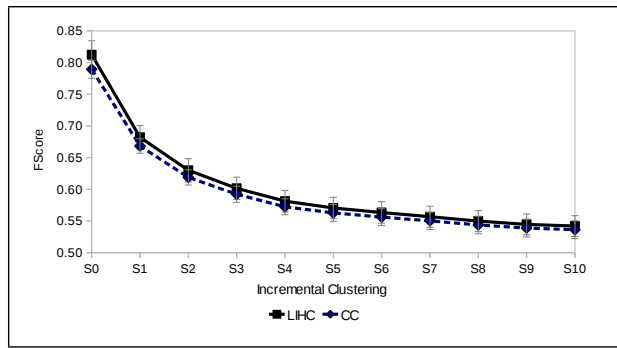

((e)) NSF

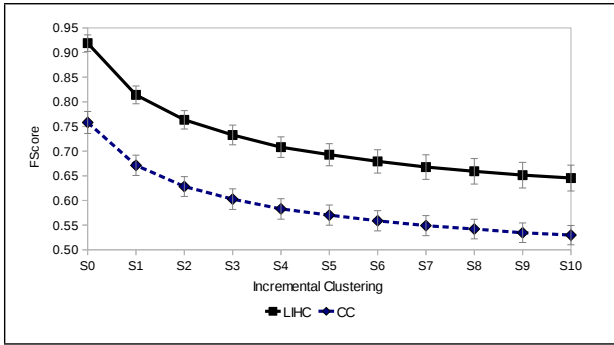

((g)) PubMed-Cancer

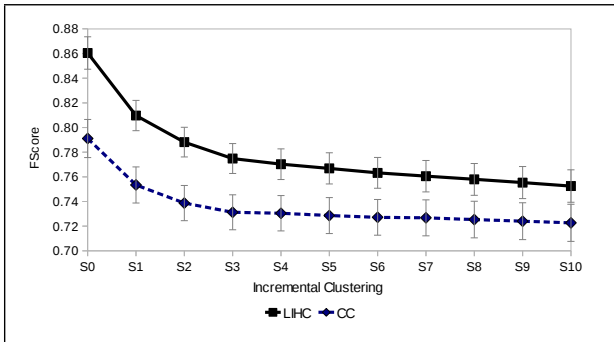

((i)) Reviews

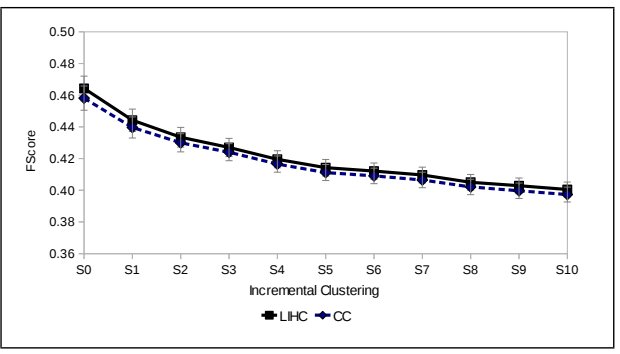

((b)) ACM

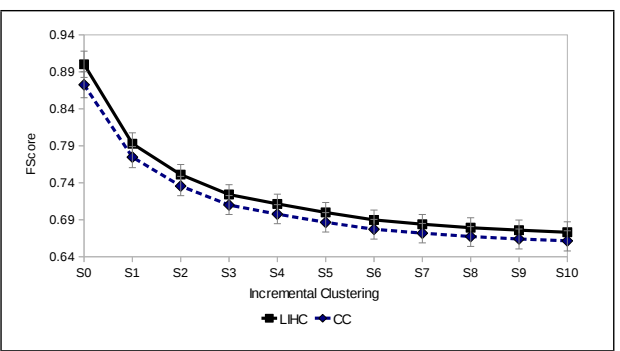

((d)) LATimes

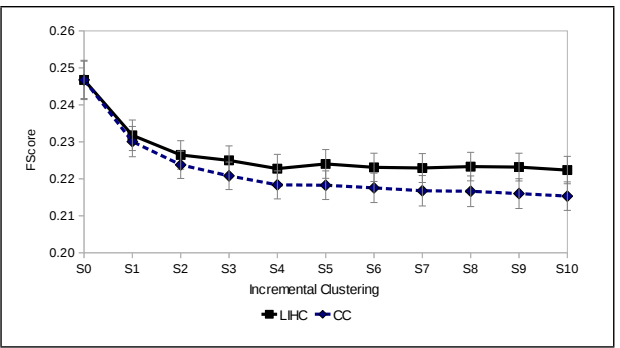

((f)) Ohsumed400

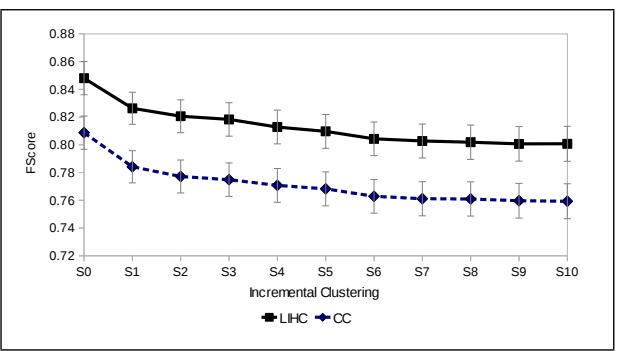

((h)) $\operatorname{Re} 8$

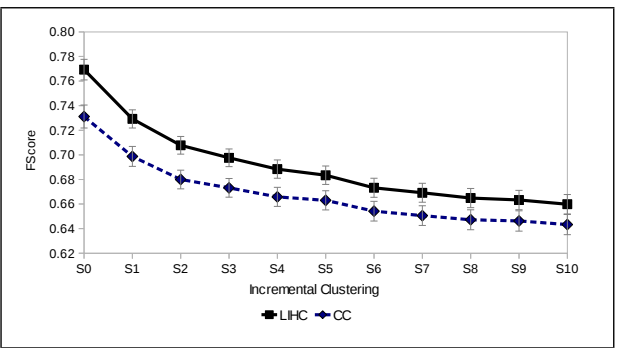

((j)) WebACE

Figura 3.6: Comparação do índice $F_{S C O R E}$ entre as abordagens $L I H C$ e CC durante o processo de agrupamento. A etapa $S_{0}$ representa a acurácia do modelo inicial e $S_{1} 0$ a acurácia da solução final de agrupamento. 
similares, no entanto, a degradação do modelo durante a etapa incremental é menor na solução obtida pela abordagem LIHC.

Para verificar a significância estatística dos resultados, utilizou-se as acurácias médias da solução final de agrupamento $\left(S_{10}\right)$, considerando diversas execuções em cada coleção textual. Neste caso, aplicou-se o teste estatístico de Wilcoxon com 95\% de confiança, conforme a recomendação de Demšar (2006) quando há dois algoritmos e múltiplas bases de dados. A análise estatística revela que a abordagem LIHC é superior ao CC, indicando que a incorporação de informação privilegiada no aprendizado do modelo de agrupamento inicial melhora a solução final do agrupamento.

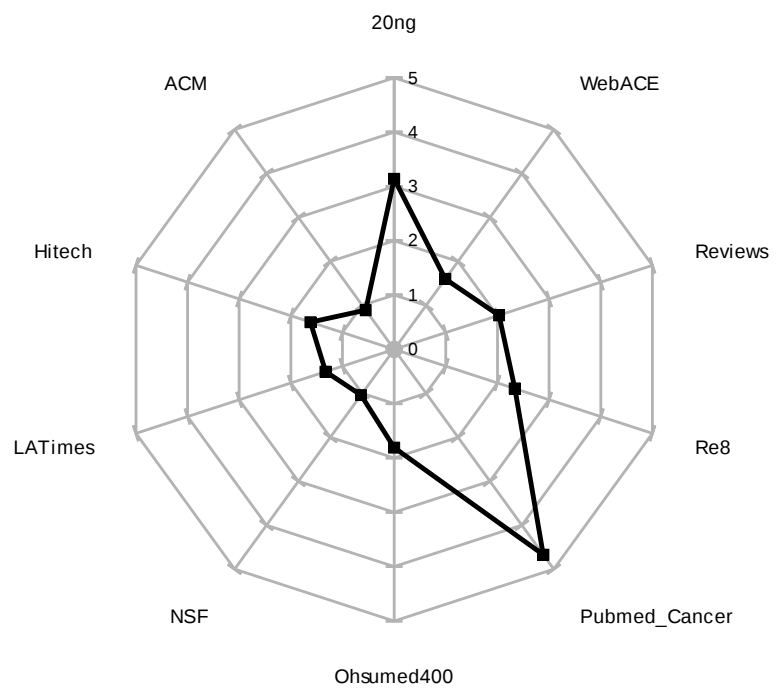

Figura 3.7: Nivel de melhoria da solução de agrupamento obtida pela abordagem LIHC (LUPI-Based Incremental Hierarchical Clustering) em relação ao CC (Consensus Clustering - sem informação Privilegiada). Para facilitar a visualização, o valor do nível é calculado por meio da raiz quadrada da porcentagem de melhora obtida pelo LIHC.

Finalmente, para ilustrar o aumento da acurácia obtida pela abordagem LIHC em relação ao CC, foi calculada a porcentagem de melhora considerando as médias do índice $F_{S C O R E}$ da etapa final do agrupamento $\left(S_{10}\right)$. Para facilitar a visualização, no gráfico da Figura 3.7, o nível de melhoria é apresentado por meio da raiz quadrada dos valores de porcentagem. As coleções que apresentaram menores niveis de melhoria foram a $A C M$ e $N S F$, com aproximadamente $1 \%$. Já as coleções PubMed - Cancer e $20 n g$ apresentaramos maiores níveis de melhoria, com $21.8 \%$ e $9.9 \%$ respectivamente. 


\subsection{Considerações Finais}

Neste capítulo foi apresentada a abordagem LIHC (LUPI-Based Incremental Hierarchical Clustering), proposta para incorporar informação privilegiada no aprendizado não supervisionado de máquina, particularmente, em tarefas de agrupamento hierárquico de textos. Foram discutidos alguns tipos de representação estruturada para dados textuais, complementares ao tradicional bag-of-words (informação técnica), bem como estudos para extrair termos compostos dos textos. Entre os estudos analisados, a representação baseada em itemsets se mostrou promissora em tarefas de agrupamento.

Uma proposta apresentada neste trabalho foi a extração de informação privilegiada a partir dessas representações complementares. Para tal, foi proposta e desenvolvida a ferramenta $\mathrm{AL}^{2} \mathrm{FIC}$, que permite aos usuários especialistas indicarem termos significativos do domínio, com apoio de seleção interativa de termos. A abordagem LIHC foi avaliada considerando esse tipo de informação privilegiada e os resultados experimentais indicam um aumento da acurácia das soluções de agrupamento, mesmo quando poucos itemsets são selecionados pelo usuário.

Além da melhoria na acurácia dos modelos de agrupamento analisada por meio de significância estatística, também é interessante analisar os resultados no contexto da significância prática. Conforme discutido na Seção 3.2, o uso da informação privilegiada para complementar a informação técnica além de permitir um aumento da acurácia, também atua no quesito da compreensão do modelo obtido, por exemplo, usando itemsets para descrição dos agrupamentos em tarefas de análise exploratória de coleções textuais. A discussão sobre a significância prática na incorporação de informação privilegiada em tarefas de agrupamento é discutida de forma mais detalhada no Capítulo 6, onde foram desenvolvidas e analisadas dois tipos aplicações que se beneficiam das abordagens investigadas nesta tese: (i) análise exploratória de mídias sociais e (ii) sistemas de recomendação sensíveis ao contexto.

Algumas das contribuições apresentadas neste capítulo foram divulgadas por meio de publicações em conferências e periódicos:

- O estudo envolvendo agrupamento de itemsets e seleção interativa de termos, que são a base da ferramenta $\mathrm{AL}^{2} \mathrm{FIC}$, foi publicado na 21st International Conference on Pattern Recognition (ICPR) (Marcacini et al., 2012b);

- O estudo sobre estratégias de consenso de agrupamentos para cenários incrementais, que investiga o aprendizado de modelos iniciais mais ro- 
bustos e sua relação com aprendizado incremental, foi publicado no 21st Brazilian Symposium on Artificial Intelligence (SBIA/BRACIS), onde o artigo foi agraciado com uma menção honrosa (Marcacini et al., 2012c);

- O estudo sobre a incorporação de seleção interativa de termos em consenso de agrupamentos e o efeito do Fator de Contribuição no consenso de agrupamentos foi submetido ao periódico Pattern Recognition Letters (Correa et al., 2014). Um estudo preliminar desses resultados foi publicado na 22nd International Conference on World Wide Web Companion (WWW) (Marcacini et al., 2013);

- A abordagem LIHC (Learning Using Privileged Information) foi introduzida em uma publicação no ACM Symposium on Document Engineering (DocEng) (Marcacini e Rezende, 2013);

- Como em cenários reais novos documentos podem ser inseridos na coleção textual para realização do agrupamento incremental, um estudo sobre pré-processamento de textos para agrupamento incremental foi publicado no X Encontro Nacional de Inteligência Artificial e Computacional (ENIAC) (Rossi et al., 2013b); e

- Um estudo das técnicas de obtenção de amostras significativas de documentos em cenários incrementais (que são úteis para o aprendizado do modelo inicial) foi publicado no Journal of Information and data Management (JIDM) (Bonin et al., 2014).

Neste primeiro momento, a extensão do paradigma LUPI para tarefas de agrupamento foi realizada por meio da abordagem LIHC, com uso de consenso de agrupamentos. No entanto, a literatura apresenta outras estratégias interessantes para esta finalidade, como utilizar a informação privilegiada para aprendizado de métricas e também para seleção de modelos de agrupamentos. Essas estratégias são discutidas nos próximos capítulos. 
4

\section{Informação Privilegiada em Aprendizado de Métricas}

\subsection{Considerações Iniciais}

A definição de uma boa medida de distância entre os documentos é crucial para a eficácia de muitos algoritmos de aprendizado de máquina (Yang, 2007; Kulis, 2013). Por exemplo, tanto em aprendizado supervisionado, como classificadores $k$-nn, quanto em aprendizado não supervisionado, como o agrupador $k$-means, há uma variedade de estudos demonstrando que a escolha da medida de proximidade apropriada resulta em substancial melhora, respectivamente, nas tarefas de classificação (Lebanon, 2006; Yang et al., 2006; Weinberger e Saul, 2009; Parameswaran e Weinberger, 2010) e agrupamento de textos (Xing et al., 2002; Bilenko et al., 2004; Baghshah e Shouraki, 2009; Wang et al., 2010).

Nesse contexto, o aprendizado de métricas visa obter uma medida de proximidade de acordo com as propriedades específicas de um conjunto de dados (Kulis, 2013). De forma geral, a estratégia utilizada para aprendizado de métricas depende da informação externa existente sobre os dados. Na existência de uma amostra de dados rotulados, espera-se obter uma medida que minimize a distância entre documentos de uma mesma classe. Já em tarefas de agrupamento, a informação externa sobre os dados é comumente representada por meio de um conjunto de restrições Must-Link e Cannot-Link que definem, respectivamente, pares de documentos que devem ser alocados em um mesmo grupo e pares de documentos que devem ser alocados em grupos diferentes. Nesse caso, espera-se obter uma medida que minimize o número de restrições violadas. Já nos cenários em que não há uma informação externa sobre 
os dados, o aprendizado de métricas tem a função de obter representações dos dados com baixa dimensionalidade que preservem as principais relações geométricas existentes na representação original.

Com a existência da informação privilegiada a respeito de uma parcela dos documentos, novas abordagens para aprendizado de métricas foram investigadas (Vapnik e Vashist, 2009; Fouad et al., 2013). Nesse caso, o objetivo do aprendizado de métricas é capturar as propriedades geométricas existentes no conjunto de documentos representados no espaço de informação privilegiada e utilizá-las para refinar as relações de proximidade entre documentos representados no espaço de informação técnica. Vapnik e Vashist (2009) propuseram uma primeira abordagem, aqui denominada de IPIML (Incorporating Privileged Information by Metric Learning), em que a informação privilegiada é utilizada para definir um fator de relevância de cada atributo existente no espaço de informação técnica. Esses fatores de relevância são utilizados para alterar a relação de proximidade entre documentos representados pela informação técnica. A abordagem IPIML foi utilizada em tarefas de classificação binária, para as quais o bem conhecido método SVM (Support Vector Machine) foi estendido para lidar com informação privilegiada, resultando no método SVM+ (Pechyony e Vapnik, 2010). O uso do SVM+ para classificação multiclasse foi investigado recentemente no trabalho de Fouad et al. (2013) ${ }^{1}$.

A abordagem IPIML tem obtido resultados promissores em tarefas de classificação, especialmente no método $S V M+$. No entanto, seu uso em tarefas de agrupamento é um aspecto ainda não explorado na literatura. Neste capítulo, é apresentada uma abordagem para incorporar informação privilegiada em tarefas de agrupamento hierárquico de documentos baseada em aprendizado de métricas. Na abordagem proposta neste trabalho, a informação privilegiada é utilizada para extrair um conjunto de restrições Must-Link, ou seja, são identificados os pares de documentos mais similares do espaço de informação privilegiada. As restrições são utilizadas para aprender medidas de proximidades no espaço de informação técnica visando respeitar tal conjunto de restrições. Em um primeiro cenário, é explorado o uso de uma medida global de forma similar à abordagem IPIML proposta por Vapnik e Vashist (2009), no qual a métrica é empregada, no contexto deste trabalho, para agrupamento de todo o conjunto de documentos. Um segundo cenário foi proposto neste trabalho, denominado IPIML-LOCAL, na qual medidas locais são aprendidas de

\footnotetext{
${ }^{1}$ Classificação multiclasse se refere à atribuição de um único rótulo $L_{i} \in\left\{L_{1}, L_{2}, \ldots, L_{k}\right\}$, de um conjunto de $k$ rótulos, para o exemplo durante a classificação. É diferente da classificação multirrótulo, em que dois ou mais rótulos podem ser atribuídos a um único exemplo.
} 
acordo com o nivel da hierarquia. Nas abordagens propostas não foram exploradas as restrições do tipo Cannot-Link, já que podem levar à intratabilidade do problema especialmente em agrupamento hierárquico.

Uma análise experimental foi realizada em diversas coleções textuais e os resultados indicam que a medida de proximidade aprendida a partir da informação privilegiada leva à soluções de agrupamento superiores quando comparado com o uso de uma medida tradicional. Em especial, a abordagem IPIML-LOCAL apresenta significativa melhora na qualidade do agrupamento hierárquico, fornecendo evidências de que medidas locais específicas para cada subgrupo de documentos são mais eficazes em capturar as propriedades geométricas existentes no espaço de informação privilegiada.

\subsection{Trabalhos Relacionados}

Os trabalhos relacionados com a abordagem aqui proposta são dividos em duas subseções. Na primeira será apresentada a abordagem IPIML proposta por Vapnik e Vashist (2009) e sua extensões mais recentes. Na segunda serão discutidos trabalhos envolvendo aprendizado de métricas em agrupamento de dados e suas conexões com abordagens relacionadas à incorporação de uma informação externa durante o aprendizado de métricas.

\subsubsection{A abordagem IPIML}

A abordagem IPIML utiliza como entrada o subconjunto de documentos que estão descritos em ambas as representações $\chi$ (informação técnica) e $\chi^{*}$ (informação privilegiada). Assim, considere o conjunto com $m$ documentos

$$
X=\left\{\left(x_{1}, x_{1}^{*}\right), \ldots,\left(x_{m}, x_{m}^{*}\right)\right\}, x_{i} \in \chi, x_{i}^{*} \in \chi^{*},
$$

e que $|\chi|$ e $\left|\chi^{*}\right|$ representam, respectivamente, a quantidade de características (atributos) nos espaços $\chi \mathrm{e} \chi^{*}$. Ainda, considere que $a_{i, j}^{*}$ é distância Euclidiana entre um par de documentos $x_{i}^{*}$ e $x_{j}^{*}$ no espaço de características $\chi^{*}$, definido na Equação 4.1,

$$
a_{i, j}^{*}=\sqrt{\sum_{w=1}^{\left|\chi^{*}\right|}\left(x_{i}^{*}(w)-x_{j}^{*}(w)\right)^{2}}
$$

em que $x_{i}^{*}(w)$ é o valor da característica $w$ para o documento $x_{i}^{*}$. 
A partir da Equação 4.1, a matriz de distâncias

$$
M^{*}=\left[\begin{array}{ccc}
a_{1,1}^{*} & \ldots & a_{1, m}^{*} \\
\vdots & \ddots & \vdots \\
a_{m, 1}^{*} & \ldots & a_{m, m}^{*}
\end{array}\right]
$$

é obtida, representando a distância entre cada par de documentos descritos no espaço de características de informação privilegiada.

De forma similar, a distância euclidiana entre cada par de documentos $x_{i} \mathrm{e}$ $x_{j}$ descritos no espaço de características de informação técnica é definida na Equação 4.2. No entanto, note que no espaço métrico em $\chi$ existem $|\chi|$ parâmetros fixos, representados pelo vetor $\vec{\lambda}$, que atuam como fatores de escala para a distância Euclidiana.

$$
a_{i, j}=\sqrt{\sum_{w=1}^{|\chi|}\left(x_{i}(w)-x_{j}(w)\right)^{2} \lambda(w)}
$$

A partir da Equação 4.2, a matriz de distâncias

$$
M=\left[\begin{array}{ccc}
a_{1,1} & \ldots & a_{1, m} \\
\vdots & \ddots & \vdots \\
a_{m, 1} & \ldots & a_{m, m}
\end{array}\right]
$$

é obtida para representar a distância entre cada par de documentos descritos no espaço de características de informação técnica.

O principal objetivo da abordagem IPIML é selecionar os parâmetros em $\vec{\lambda}$ de forma a aproximar as matrizes $M^{*}$ e $M$ de acordo com a Equação 4.3.

$$
\rho=\sum_{i, j=1}^{m}\left(a_{i, j}^{2}-\left(a_{i, j}^{*}\right)^{2}\right)^{2}
$$

A Equação 4.4 expressa a forma explícita do critério de proximidade $\rho$ entre $M^{*}$ e $M$, em função de um determinado vetor $\vec{\lambda}$.

$$
\rho(\vec{\lambda})=\sum_{i, j}^{m}\left(\sum_{w=1}^{|\chi|} \lambda(w)\left(x_{i}(w)-x_{j}(w)\right)^{2}-\left(a_{i, j}^{*}\right)^{2}\right)^{2}
$$

Ao definir um vetor $\vec{\lambda}$ que maximiza a proximidade entre $M$ e $M^{*}$, a distância entre os documentos no espaço de características de informação técnica é adaptada considerando as propriedades geométricas existentes no espaço de informação privilegiada.

Para selecionar os parâmetros em $\vec{\lambda}$, a Equação 4.4 pode ser reescrita na sua forma quadrática: 


$$
\sum_{w=1}^{|\chi|} \lambda(w) c(w)-\frac{1}{2} \sum_{w, l=1}^{|\chi|} \lambda(w) \lambda(l) d(w, l)
$$

sujeita à restrição $\lambda(w) \geq 0$, em que $c(w)$ e $d(w, l)$ são definidos, respectivamente, por:

$$
c(w)=\sum_{i, j=1}^{m}\left(x_{i}(w)-x_{j}(w)\right)^{2}\left(a_{i, j}^{*}\right)^{2}
$$

e

$$
d(w, l)=\sum_{i, j=1}^{m}\left(x_{i}(w)-x_{j}(w)\right)^{2}\left(x_{i}(l)-x_{j}(l)\right)^{2}
$$

O vetor $\vec{\lambda}$ é obtido como resultado da maximização da forma quadrática acima por meio de alguma técnica de otimização para programação quadrática (Floudas e Pardalos, 2006).

Uma vez obtidos os valores dos parâmetros em $\vec{\lambda}$, as representações vetoriais dos documentos descritos no espaço de informação técnica são transformadas para a forma $x_{i}^{\lambda}=\left(\sqrt{\lambda(1)} x_{i}(1), \ldots, \sqrt{\lambda(w)} x_{i}(w)\right)$, com $w=|\chi|$, em que $x_{i}(l)$ indica o valor da característica $l$ para o documento $x_{i}$. Isto significa que o valor de cada característica dos documentos no espaço de informação técnica foi ponderado de acordo com o conceito de distância no espaço de informação privilegiada, ou seja, os parâmetros em $\vec{\lambda}$ incorporam a informação privilegiada no problema.

Vapnik e Vashist (2009) mostraram que o conjunto de dados de informação técnica na forma $X^{\lambda}=\left\{x_{1}^{\lambda}, \ldots, x_{m}^{\lambda}\right\}$ pode ser utilizado em classificadores $S V M$, durante o treinamento do modelo preditivo, para incorporar a informação privilegiada. Uma limitação da abordagem IPIML proposta por Vapnik e Vashist (2009) é que o problema de otimização definido na Equação 4.4 para identificar os parâmetros $\vec{\lambda}$ possui alto custo computacional e se torna inviável para dados de alta dimensionalidade, como ocorre em bases de dados textuais. Uma possível solução para esta limitação é investigada e discutida em Fouad (2013), na qual são utilizados um conjunto de protótipos para representar exemplos de cada classe. De forma geral, o objetivo é encontrar os parâmetros que minimizam a distância entre exemplos e protótipos de uma mesma classe. 
Como o número de protótipos é muito inferior ao número de exemplos, então o processo se torna menos computacionalmente custoso do que a abordagem de Vapnik e Vashist (2009), que tenta maximizar a correspondência entre duas matrizes de distâncias (Fouad et al., 2013). Uma versão do método de aprendizado de métricas baseada em protótipos para classificação com dados ordinais também foi proposta em Fouad e Tino (2013). Embora essas soluções permitam melhorar a eficiência da abordagem IPIML originalmente proposta, elas são específicas para aprendizado supervisionado, não sendo diretamente aplicáveis em tarefas de agrupamento.

\subsubsection{Aprendizado de Métricas em Agrupamento de Dados}

Existe uma vasta literatura relacionada ao aprendizado de métricas, com diferentes paradigmas e aplicações (Kulis, 2013). Nesta seção serão discutidos os principais trabalhos envolvendo aprendizado de métricas para agrupamento de textos, uma vez que a alta dimensionalidade desse tipo de dado necessita de abordagens mais específicas (Lebanon, 2006).

De forma geral, dada uma função de distância $d(\vec{x}, \vec{y})$ entre dois documentos $\vec{x}$ e $\vec{y}$, o aprendizado de métricas visa obter uma nova função de distância $\widetilde{d}(\vec{x}, \vec{y})$, que obtenha soluções de agrupamento melhores do que a função de distância original (Kulis, 2013). A função de distância $\widetilde{d}(\vec{x}, \vec{y})$ pode ser reescrita na forma $d(f(\vec{x}), f(\vec{y}))$ para alguma função $f$, ou seja, o aprendizado de métricas obtém uma função responsável por mapear os dados e a função de distância original é aplicada a partir dos dados mapeados.

Um dos principais trabalhos sobre aprendizado de métricas em tarefas de agrupamento foi proposto por Xing et al. (2002). A motivação dos autores era investigar a seguinte questão: suponha que o usuário indique certos exemplos do conjunto de dados que ele considera similares, então é possível automaticamente aprender uma medida que respeita essas relações de similaridade, ou seja, que defina valores baixos de distância entre os pares considerados similares? Essa questão foi explorada por meio de um problema de otimização definido na Equação 4.5. Neste problema, $(\vec{x}, \vec{y}) \in \mathcal{S}$ se $\vec{x}$ e $\vec{y}$ são considerados similares pelo usuário, formando um conjunto $\mathcal{S}$ de pares de exemplos similares. Ainda, considere que a distância entre dois exemplos é calculada de acordo com a equação $d_{\mathbf{A}}(\vec{x}, \vec{y})=\|\vec{x}-\vec{y}\|_{\mathbf{A}}^{2}=\sqrt{(\vec{x}, \vec{y})^{T} \mathbf{A}(\vec{x}, \vec{y})}$. 


$$
\begin{aligned}
& \operatorname{Min}_{\mathbf{A}} \sum_{(\vec{x}, \vec{y}) \in \mathcal{S}}\left\|\vec{x}_{i}-\vec{x}_{j}\right\|_{\mathbf{A}}^{2} \\
& \text { sujeito a } \sum_{(\vec{x}, \vec{y}) \in \mathcal{D}}\|\vec{x}-\vec{y}\|_{\mathbf{A}} \geq 1,
\end{aligned}
$$

$$
\mathbf{A} \succeq 0
$$

A ideia básica é encontrar os valores de A para que os pares de exemplos similares tenham uma baixa distância entre eles. Um solução trivial, mas não útil, é a solução $\mathbf{A}=0$. Assim, a restrição $\mathbf{A} \succeq 0$ é necessária, ou seja, a matriz A deve ser positiva semidefinida. Já a restrição $\sum_{(\vec{x}, \vec{y}) \in \mathcal{D}}\|\vec{x}-\vec{y}\|_{\mathbf{A}} \geq 1$ garante que a matriz A não seja uma solução que transforme o conjunto de dados em um único ponto. Nesse caso, o conjunto $\mathcal{D}$ representa pares de exemplos definidos como não similares (ou todos os pares não presentes no conjunto $\mathcal{S}$ ), e o valor 1 é apenas uma constante que pode ser substituída por qualquer valor real positivo. É importante ressaltar que quando A for a matriz identidade, então a distância entre os exemplos é a distância euclidiana. Por outro lado, quando A é uma matriz diagonal, então os valores $\left(\mathbf{A}_{11}, \mathbf{A}_{22}, \ldots, \mathbf{A}_{l l}\right)$ representam pesos para cada uma das $l$ características do conjunto de dados. Se a matriz A for completa, então novas características são geradas de acordo com a combinação linear das características originais.

Uma das principais vantagens da abordagem de aprendizado de métricas propostas por Xing et al. (2002) é tratar o aprendizado de métricas como um problema de otimização convexo, podendo ser resolvido por algoritmos eficientes de otimização matemática. Por outro lado, a nova função de distância $\widetilde{d}(\vec{x}, \vec{y})=d_{\mathbf{A}}(\vec{x}, \vec{y})$, tecnicamente, é uma pseudométrica, pois não há garantias que $d_{\mathbf{A}}(\vec{x}, \vec{y})=0$ implica sempre em $\vec{x}=\vec{y}$ (Garber e Hazan, 2011). Mesmo com esta limitação, as avaliações experimentais realizadas obtiveram resultados promissores em relação à acurácia do agrupamento, motivando outros trabalhos na área.

O algoritmo MPCK-Means, proposto por Bilenko et al. (2004), incorpora aprendizado de métricas a partir de restrições Must Link e Cannot-Link para agrupamento particional. O MPCK-Means tem dois diferenciais: a primeira é a capacidade de aprender uma medida de proximidade por grupo da partição e a segunda é a possibilidade de violar restrições consideradas ruidosas ou muito discrepantes em relação às propriedades geométricas do conjunto de dados. Para obter uma medida por grupo, o MPCK-Means executa o processo de aprendizado de métricas a cada iteração do algoritmo de agrupamento, que 
é baseado no $k$-means. Assim, em cada iteração, são considerados apenas as restrições relacionadas aos exemplos de cada um dos $k$ grupos para obter $k$ medidas. Já a violação das restrições é baseada em um esquema de penalidades que é somada na função objetivo do $k$-means. Por exemplo, uma restrição pode ser violada se a função objetivo (a ser minimizada) obtém um valor menor do que mantendo tal restrição, mesmo adicionando um fator de penalização (Bilenko et al., 2004). Recentemente, Covões et al. (2013) apresentaram uma robusta avaliação empírica de algoritmos de agrupamento com restrições que permitem a violação de restrições. Um dos resultados interessantes desta avaliação é que aprender uma medida por grupo não necessariamente leva à soluções superiores quando comparado com uso de uma medida global para todo o agrupamento, particularmente para o MPCK-Means que incorpora o aprendizado de métricas durante as iterações do agrupamento. Por outro lado, o uso de medidas locais obtidas antes do processo de agrupamento a partir de um conjunto de dados rotulados, ou seja, aprendizado de uma medida por classe, têm apresentado resultados promissores (Yang et al., 2006; Kulis, 2013).

A abordagem LMNN (Large Margin Nearest Neighbor Metric) (Blitzer et al., 2005; Weinberger e Saul, 2009) visa melhorar o resultado de classificação do algoritmo $k-n n$, mas que também foi aplicada em tarefas de agrupamento (Hauberg et al., 2012). Para cada exemplo é calculado um perímetro considerando seus $k$ vizinhos de mesma classe. Os exemplos de classes diferentes que estão neste perímetro, chamados de impostores, são penalizados. O aprendizado de métrica tem a função minimizar a distância entre os exemplos de mesma classe do perímetro, ao mesmo tempo que penaliza (aumenta a distância) os exemplos impostores. O $L M N N$ também permite aprender medidas locais, uma vez que podem ser consideradas apenas as restrições relacionadas aos exemplos inseridos em um perímetro para o aprendizado de métricas. Por outro lado, ao contrário das abordagens anteriores, baseadas em conjunto de restrições Must-Link e Cannot-Link, a abordagem LMNN necessita conhecer a classe dos exemplos.

\subsubsection{Análise Conceitual}

Embora diferentes abordagens para aprendizado de métricas tenham sido propostas, há nelas um objetivo em comum que é obter uma medida de distância da família de Mahalanobis. A distância de Mahalanobis entre $\vec{x}$ e $\vec{y}$ é $d_{\text {mahal }}(\vec{x}, \vec{y})=\sqrt{(\vec{x}-\vec{y})^{T} \Sigma^{-1}(\vec{x}-\vec{y})}$, em que $\Sigma^{-1}$ representa a matriz de cova- 
riância dos dados. No entanto, esta matriz de covariância não é conhecida, geralmente sendo estimada por meio de uma amostra dos dados. O aprendizado de métricas visa obter uma matriz positiva semidefinida A que irá atuar como a matriz $\Sigma$ da distância de Mahalanobis.

O efeito prático da matriz A é definir a importância de cada característica (ou combinação de características) na nova medida de distância. Em uma comparação com o método IPIML de Vapnik e Vashist (2009), quando a matriz A é diagonal então esta tem a mesma aplicação do vetor de parâmetros $\vec{\lambda}$. Assim, propostas de aprendizado de métricas para agrupamento já existentes na literatura também podem ser utilizadas para incorporar informação privilegiada, com a vantagem de que o problema é tratado com uso de algoritmos para programação convexa.

\subsection{Abordagens Propostas: IPIML-GLOBAL e IPIML-LOCAL}

A incorporação de informação privilegiada por meio de aprendizado de métricas tem sido explorada em cenários supervisionados, particularmente com a abordagem IPIML para o classificador SVM+. Por outro lado, a literatura envolvendo aprendizado de métricas para agrupamento de dados apresenta abordagens promissoras, principalmente àquelas que visam extrair um subconjunto de medidas de distância da família da Mahalanobis.

Em vista disso, as abordagens propostas neste capítulo exploram a estratégia de aprendizado de métricas para agrupamentos a partir de conjuntos de restrições. Uma das principais motivações é a formulação e resolução do problema por meio de programação convexa, permitindo o uso de algoritmos eficientes de otimização matemática.

Em um primeiro momento, é apresentada uma técnica para identificar e extrair restrições significativas a partir do espaço de informação privilegiada. Essas restrições são utilizadas para aprendizado de medidas de proximidade considerando a tarefa de agrupamento hierárquico. A primeira abordagem, denominada IPIML-GLOBAL, visa aprender uma única medida que é utilizada durante todo processo de agrupamento. A segunda abordagem, denominada IPIML-LOCAL, visa aprender um conjunto de medidas, uma para cada subgrupo, considerando os diferentes níveis de granularidade do agrupamento hierárquico. 


\subsubsection{Extração de Restrições Must-Link a partir de Informação Privilegiada}

A estratégia de aprendizado de métricas aqui investigada é baseada em um conjunto de restrições sobre os dados. A maioria dos trabalhos existentes assume que este conjunto de restrições é informado pelo usuário. No entanto, em aplicações práticas a definição do conjunto de restrições é uma tarefa árdua para o usuário, uma vez que ele usualmente não conhece as propriedades geométricas do conjunto de dados, especialmente em dados com alta dimensionalidade.

A existência de informação privilegiada a respeito de uma parcela dos textos é uma alternativa promissora para extração automática de um conjunto de restrições. A ideia básica é explorar as principais estruturas de agrupamento existentes no espaço de informação privilegiada e representar tais estruturas por meio de restrições do tipo Must-Link. Assim, se dois documentos apresentaram alto grau de similaridade no espaço de informação privilegiada, então uma restrição Must-Link é gerada entre eles para que a futura medida represente esta relação no espaço de informação técnica.

Para a extração de restrições Must-Link, considere um conjunto de $m$ documentos $X=\left\{\left(\vec{x}_{1}, \vec{x}_{1}^{*}\right), \ldots,\left(\vec{x}_{m}, \vec{x}_{m}^{*}\right)\right\}, \vec{x}_{i} \in \chi, \vec{x}_{i}^{*} \in \chi^{*}$, em que $\chi$ representa as características no espaço de informação técnica e $\chi^{*}$ as características no espaço de informação privilegiada. Considere também a distância Euclidiana, definida na Equação 4.6, entre dois documentos representados no espaço de caracteristicas $\chi^{*}$ :

$$
d_{p r i}^{*}\left(\vec{x}_{i}^{*}, \vec{x}_{j}^{*}\right)=\left\|\vec{x}_{i}^{*}-\vec{x}_{j}^{*}\right\|^{2}=\left(\vec{x}_{i}^{*}-\vec{x}_{j}^{*}\right)^{T}\left(\vec{x}_{i}^{*}-\vec{x}_{j}^{*}\right)
$$

em que $T$ denota a matriz transposta.

As restrições são derivadas da estrutura de agrupamento existente no espaço de informação privilegiada. Para tal, um algoritmo de agrupamento particional é executado no conjunto de documentos de informação privilegiada $X_{p r i}=\left\{\vec{x}_{i}^{*}\right\}_{i=1}^{m}$, com uso da distância definida na Equação 4.6. A partição deve ser rígida, ou seja, $P=\left\{G_{r}\right\}_{r=1}^{k}$, onde $G_{r} \cap G_{s}=\emptyset, \forall r \neq s$. O número de documentos de um grupo $G_{r}$ é definido como $\left|G_{r}\right|$. O número de grupos é definido como $k=\sqrt{m}$, que é superestimado de forma a identificar diferentes (micro) regiões de alta densidade dos dados no espaço $\chi^{*}$. Por fim, considere a existência de uma função $L(i)$ que retorna o rótulo do grupo (de 1 até $k$ ) em que o documento $\vec{x}_{i}^{*}$ está alocado. 
Uma restrição do tipo Must-Link é definida como $c_{i j}$, indicando que os documentos $\vec{x}_{i}$ e $\vec{x}_{j}$ devem ser alocados em um mesmo grupo. O conjunto $\mathcal{M}^{*}$ de todas as restrições Must-Link extraídas do espaço de informação de privilegiada é obtido pela Equação 4.7:

$$
\mathcal{M}=\left\{c_{i j} \mid L(i)=L(j) \text { e } d_{p r i}^{*}\left(\vec{x}_{i}^{*}, \vec{x}_{j}^{*}\right)<y\right\}, \forall i \neq j
$$

no qual $y$ é um limiar de distância mínima que define quando dois documentos alocados em um mesmo grupo são próximos o suficiente para formar uma restrição Must-Link. O valor de limiar $y$ depende das propriedades geométricas do conjunto de dados e é estimado automaticamente por meio da Equação 4.8.

$$
y=\frac{1}{k} \sum_{r=1}^{k} \sum_{i, j} \frac{2 \times d_{p r i}^{*}\left(\vec{x}_{i}^{*}, \vec{x}_{j}^{*}\right)}{\left(\left|G_{r}\right|\right)\left(\left|G_{r}\right|-1\right)}, \forall i \neq j, L(i)=r, L(j)=r
$$

O valor do limiar y é calculado de acordo com a média dos valores das distâncias entre todos os possiveis pares de documentos, desde que estes documentos estejam alocados no mesmo grupo. Na prática, são selecionados pares de documentos que possuem um valor significativo de proximidade.

Para exemplificar esse comportamento, na Figura 4.1 é apresentado um esquema visual do conceito da extração de restrições Must-Link. Neste caso, uma projeção bidimensional de uma base de dados com 3 classes e 150 exemplos é ilustrada na Figura 4.1A. Um grafo representando as conexões entre pares de restrições Must-Link é exibido na Figura 4.1B, em que cada aresta representa uma restrição entre dois exemplos (vértices). É possível observar que apenas os pares de exemplos mais similares foram inseridos no conjunto de restrições. No entanto, esta estratégia para extração de restrições Must-Link não é isenta de ruídos, ou seja, há restrições incorretas envolvendo exemplos de classes diferentes. Na abordagem proposta neste trabalho, a extração de restrições Must-Link pode ser refinada de acordo a granularidade do processo de agrupamento hierárquico, permitindo corrigir restrições formadas de maneira incorreta.

\subsubsection{Aprendizado de Métricas para Agrupamento Hierárquico de Documentos}

A estratégia de aprendizado de métricas aqui investigada incorpora a informação privilegiada por meio do conjunto de restrições $\mathcal{M}$ e a nova medida de proximidade é então empregada para agrupamento dos documentos repre- 


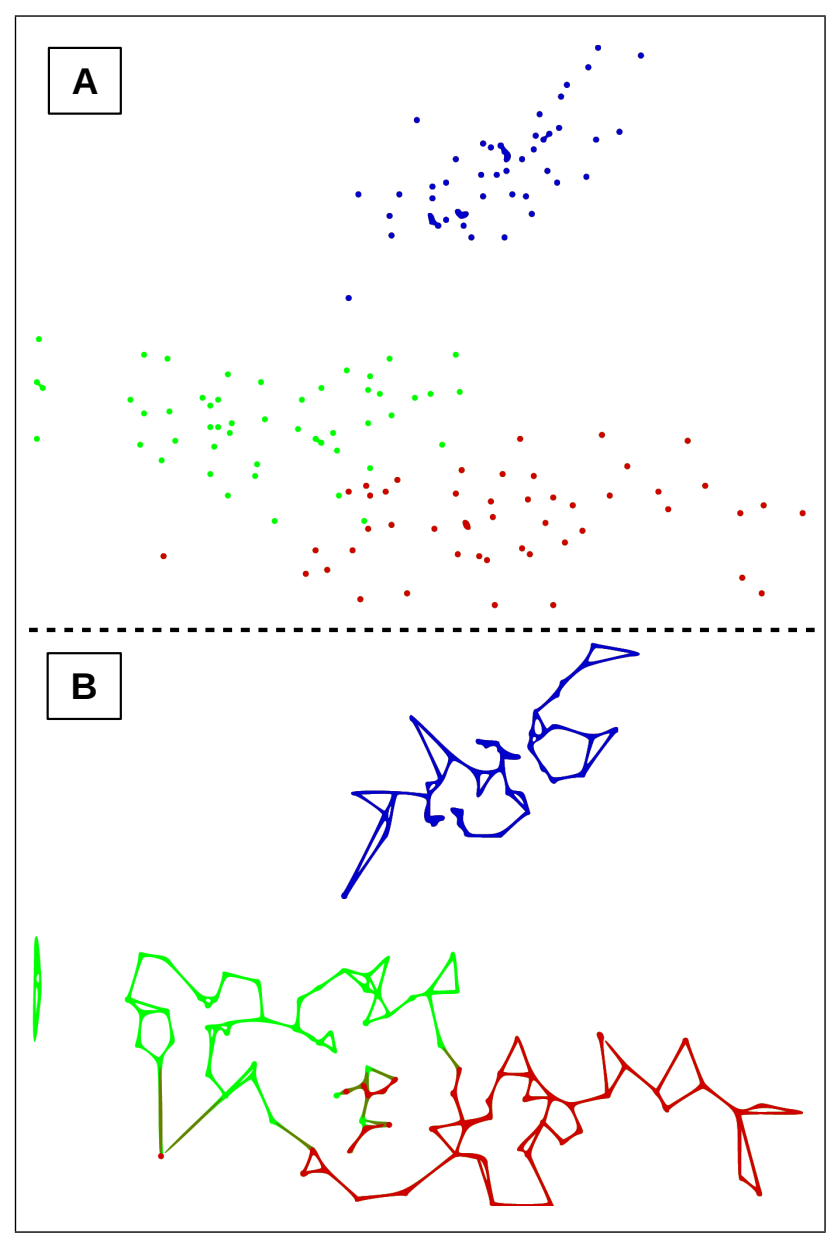

Figura 4.1: Ilustração do processo de extração de restrições do tipo Must-Link conforme a estrutura de agrupamento. No grafo, dois pontos estão conectados por uma aresta se a distância entre eles é considerada significativa.

sentados pela informação técnica. De forma geral, pode ser visto como uma versão da abordagem IPIML proposta em Vapnik e Vashist (2009) para tarefas de agrupamento. A principal diferença consiste na estratégia utilizada para incorporar restrições no aprendizado de métricas, que é baseada no problema de programação convexa de Xing et al. (2002).

Relembre que o objetivo da abordagem IPIML é extrair um vetor de parâmetros $\vec{\lambda}$ (a partir do espaço de informação privilegiada $\chi^{*}$ ) que irá ponderar a relevância das características do espaço de informação técnica $\chi$. O vetor de parâmetros $\vec{\lambda}$ é análogo à diagonal da matriz A obtida em aprendizado de medidas da família de Mahalanobis. Quando o objetivo é calcular os valores da diagonal A, então o problema de aprendizado de métricas pode se formulado conforme a Equação 4.9 (Xing et al., 2002): 


$$
g(\vec{\lambda})=g\left(\mathbf{A}_{11}, \mathbf{A}_{22}, \ldots, \mathbf{A}_{l l}\right)=\sum_{c_{i j} \in \mathcal{M}}\left\|\vec{x}_{i}-\vec{x}_{j}\right\|_{\mathbf{A}}^{2}-\log \left(\sum_{\bar{c}_{i j} \in \mathcal{C}}\left\|\vec{x}_{i}-\vec{x}_{j}\right\|_{\mathbf{A}}\right)
$$

O objetivo é minimizar $g(\vec{\lambda})$ em função dos parâmetros $\vec{\lambda}$, sujeito a $\mathbf{A} \succeq 0$. Nesta equação, a minimização do termo $\sum_{c_{i j} \in \mathcal{M}}\left\|\vec{x}_{i}-\vec{x}_{j}\right\|_{\mathbf{A}}^{2}$ representa uma pequena distância quadrática entre pares de documentos do conjunto $\mathcal{M}$, que foram extraídos a partir da informação privilegiada. Note que a minimização pode ser obtida simplesmente definindo pequenos valores para os parâmetros em $\vec{\lambda}$, o que não é uma solução útil. Nesse sentido, o termo $-\log \left(\sum_{\bar{c}_{i j} \in \mathcal{C}}\left\|\vec{x}_{i}-\vec{x}_{j}\right\|_{\mathbf{A}}\right)$ permite restringir esse comportamento adicionandose restrições do tipo Cannot-Link, representadas por pares de documentos $\bar{c}_{i j}$ no conjunto $\mathcal{C}$. A definição dessas restrições deve ser realizada de forma cuidadosa, uma vez que podem levar a intratabilidade do problema. Neste trabalho, tais restrições são compostas por pares de medoides da partição $P$ (obtida por meio da estratégia definida na seção anterior) desde que a distância entre os medoides seja maior do que o limiar $y$. Xing et al. (2002) demonstraram que o problema de minimização formulado na Equação 4.9 pode ser resolvido por algoritmos de otimização para programação convexa e representa um caso particular do método de aprendizado de métricas definido na Equação 4.5.

Duas abordagens para aprendizado de medidas com uso de informação privilegiada são propostas, considerando o cenário de agrupamento hierárquico de textos. A primeira, denominada IPIML-GLOBAL, é definida no Algoritmo 4. Neste algoritmo, o conjunto de restrições é extraído no início do processo de agrupamento, considerando todos os documentos representados pela informação privilegiada. Uma única medida de proximidade é aprendida e utilizada em uma estratégia de agrupamento hierárquico top-down, similar ao Bisecting $k$-means. Neste caso, inicialmente todos os documentos estão em um mesmo grupo, que é iterativamente dividido em dois subgrupos até a obtenção de grupos folhas (com apenas um documento).

Uma das características da abordagem IPIML-GLOBAL é que o aprendizado de uma única medida para todo o conjunto de dados, em geral, não permite satisfazer todas as restrições extraídas. Há evidências na literatura de que a extração de restrições "locais" considerando um subconjunto específico de documentos obtém melhores medidas, especialmente para tarefas de classificação com uso do $k-n n$, a exemplo da abordagem $L M N N$.

Nesse contexto, a abordagem proposta IPIML-LOCAL utiliza a estratégia de aprendizado de medidas locais. Enquanto a abordagem LMNN seleciona um 


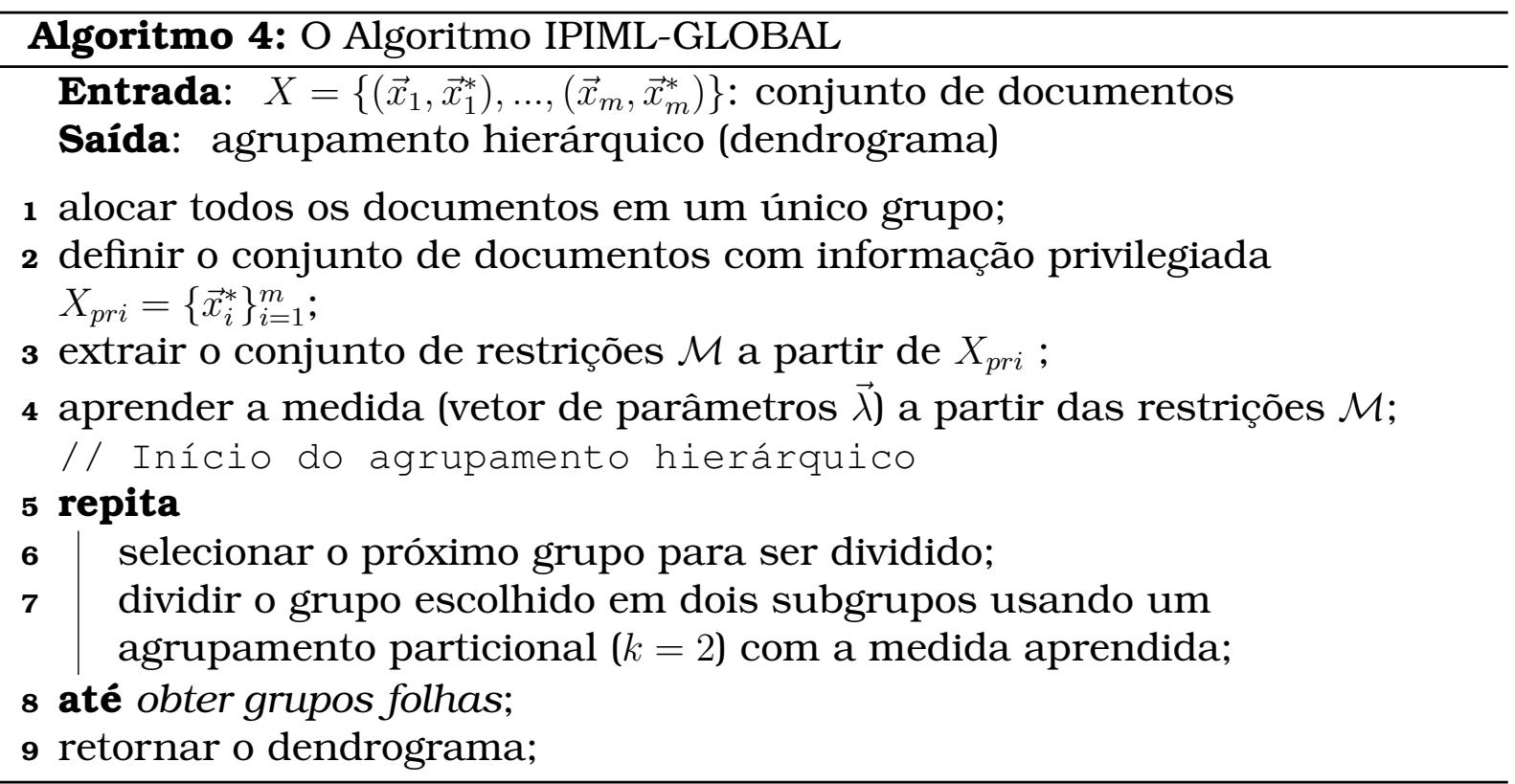

subconjunto de restrições de acordo com as classes dos documentos, a abordagem IPIML-LOCAL repete o processo de extração de restrições em um determinado subconjunto de documentos (alocados em um subgrupo) e, então, o processo de aprendizado de medidas locais considera essas novas restrições.

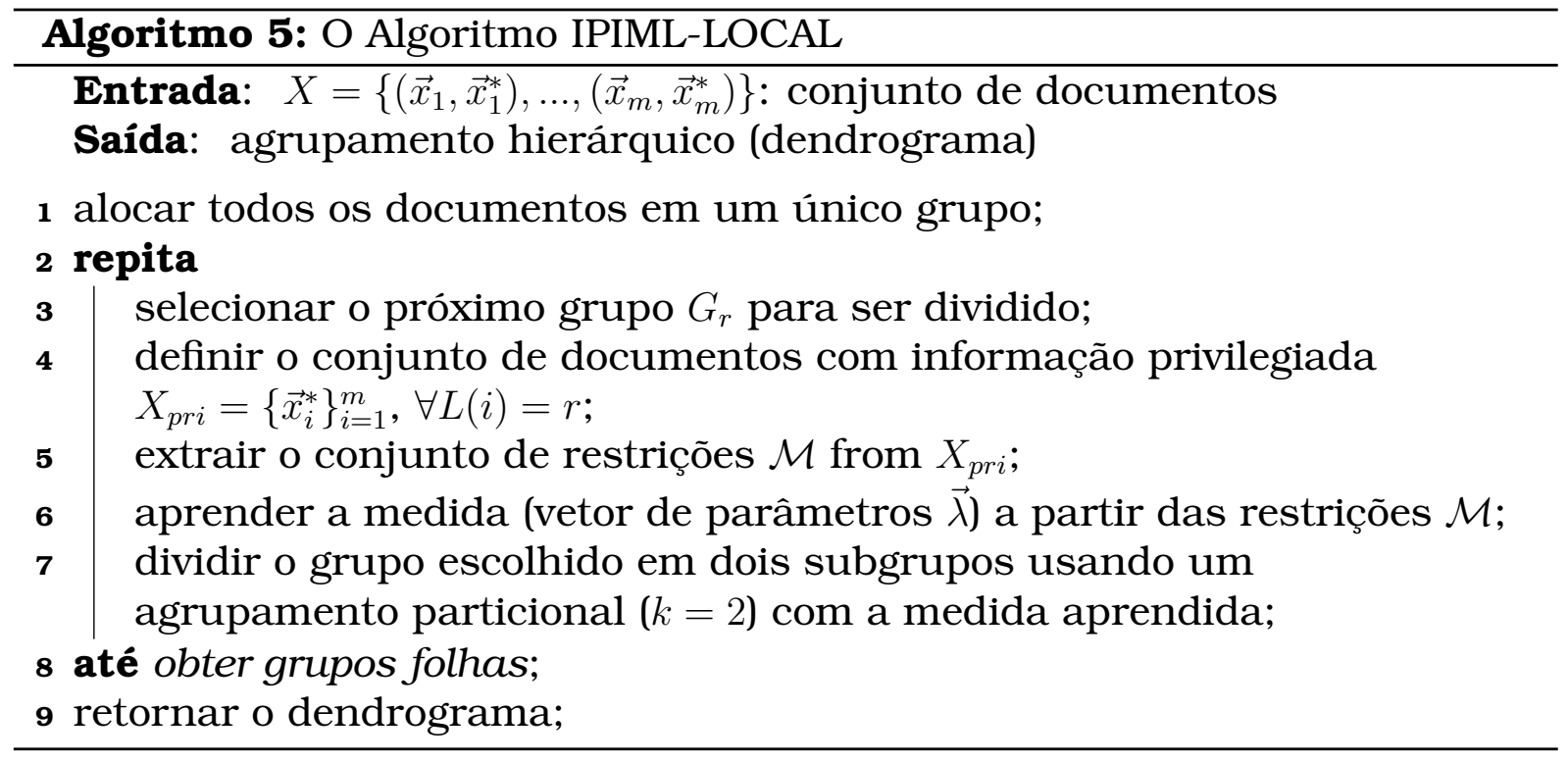

O Algoritmo 5 explora os diversos níveis de granularidade do agrupamento hierárquico para aprendizado de medidas locais. A medida obtida no grupo raiz é a mesma utilizada pelo IPIML-GLOBAL. Nas próximas iterações, na linha 4 é selecionado um subconjunto de documentos considerando um determinado subgrupo. O conjunto de restrições para aprendizado da medida local 
é extraído a partir deste subgrupo, utilizando a mesma estratégia definida na Seção 4.3.1. Dessa forma, as restrições e, consequentemente, a medida de proximidade, podem ser refinadas para cada subgrupo, permitindo a obtenção de medidas mais apropriadas a cada nível da hierarquia.

\subsection{Análise Experimental}

A abordagem IPIML-GLOBAL descrita na seção anterior representa o método mais similar existente na literatura, proposto por Vapnik e Vashist (2009), adaptado para o contexto deste trabalho. Já a abordagem proposta IPIMLLOCAL tem uma contribuição mais significativa para cenários envolvendo agrupamento hierárquico, por meio do aprendizado de medidas locais. Nesse sentido, o objetivo da avaliação experimental é, além de analisar eficácia de abordagens que incorporam informação privilegiada por meio de aprendizado de métricas em tarefas de agrupamento, também comparar as abordagens IPIML-GLOBAL e IPIML-LOCAL.

$\mathrm{Na}$ análise experimental aqui apresentada foram utilizadas as 10 coleções textuais de benchmark descritas no Capítulo 3. Foi empregado o índice $F_{S C O R E}$ para estimar a acurácia dos modelos de agrupamento. A estratégia de avaliação é baseada em duas etapas. Na primeira, um subconjunto $S_{0}$ de documentos que são representados em ambos espaços de características (informação técnica e privilegiada) é utilizado para aprendizado de um modelo inicial por meio das abordagens IPIML-GLOBAL e IPIML-LOCAL. Em seguida, o restante dos documentos, que estão representados apenas pela informação técnica, são inseridos no modelo por meio de agrupamento incremental. O processo de agrupamento incremental é avaliado em diferentes etapas por meio de dez subconjuntos (balanceados) de documentos, $S_{1}, S_{2}, \ldots, S_{10}-$ o que permite analisar a estabilidade do modelo inicial ao longo do processo de agrupamento incremental.

A estratégia de agrupamento incremental utilizada é baseada na técnica de vizinho mais próximo, na qual novos documentos são alocados aos grupos existentes conforme sua proximidade com os centroides. Nos experimentos realizados, a distância entre um novo documento e um centroide é computada por meio da medida aprendida durante a obtenção do modelo inicial. Assim, no caso da abordagem IPIML-GLOBAL, uma única medida é empregada durante todo o agrupamento incremental. Já no caso da abordagem IPIML-LOCAL, o novo documento é inserido no grupo mais próximo de acordo 
com as medidas de proximidade relativas a cada grupo. O processo de agrupamento foi repetido 100 vezes e são apresentados as respectivas médias e desvios padrão do índice $F_{S C O R E}$.

Nas Figuras 4.2 e 4.3 são apresentados os valores de $F_{S C O R E}$ dos modelos de agrupamento para as coleções textuais analisadas. Para cada coleção textual são apresentados dois gráficos. No primeiro gráfico, à esquerda, são apresentados os valores médio de $F_{S C O R E}$ desde o aprendizado do modelo inicial $\left(S_{0}\right)$ e as subsequentes etapas de agrupamento incremental $\left(S_{1}\right.$ até $\left.S_{10}\right)$. No segundo gráfico, é apresentada uma relação entre o número de restrições extraídas versus número de restrições incorretas de acordo com o nível do agrupamento hierárquico (esta relação será discutida em detalhes na análise dos resultados).

O primeiro aspecto analisado nos experimentos é a comparação entre as abordagens IPIML-GLOBAL e IPIML-LOCAL para aprendizado do modelo inicial. Com base nas coleções textuais utilizadas nos experimentos, não é possível indicar diferença significativa em relação à acurácia dos modelos obtidos pelas duas abordagens. Assim, considerando apenas o aprendizado do modelo inicial, o resultado obtido está de acordo com a análise experimental realizada por Covões et al. (2013), na qual também não foi observada diferença significativa no uso de medidas de proximidade globais e locais para agrupamento particional.

A vantagem da abordagem IPIML-LOCAL se torna mais evidente durante o processo de agrupamento incremental, na qual a degradação do modelo ao longo do agrupamento incremental é menor com uso das medidas locais. Ao analisar a solução final do agrupamento (etapa $S_{10}$ ), a abordagem IPIML-LOCAL apresenta resultados superiores em algumas bases dados, quando comparado com a abordagem IPIML-GLOBAL.

Para uma melhor compreensão do comportamento da abordagem IPIML$L O C A L$, no gráfico à direita é indicado (1) a porcentagem de restrições incorretas extraídas por grupo e (2) o número médio de restrições por grupo conforme o nível do agrupamento hierárquico (eixo $x$ ). Na análise experimental, uma restrição Must-Link é considerada incorreta se os documentos no par em questão são de classes diferentes.

No início do processo de agrupamento hierárquico (grupo raiz), a abordagem IPIML-LOCAL é similar à IPIML-GLOBAL, uma vez que usam o mesmo conjunto de documentos para extração de restrições e aprendizado da métricas. Nesse nível, também é extraída a maior porcentagem de restrições 


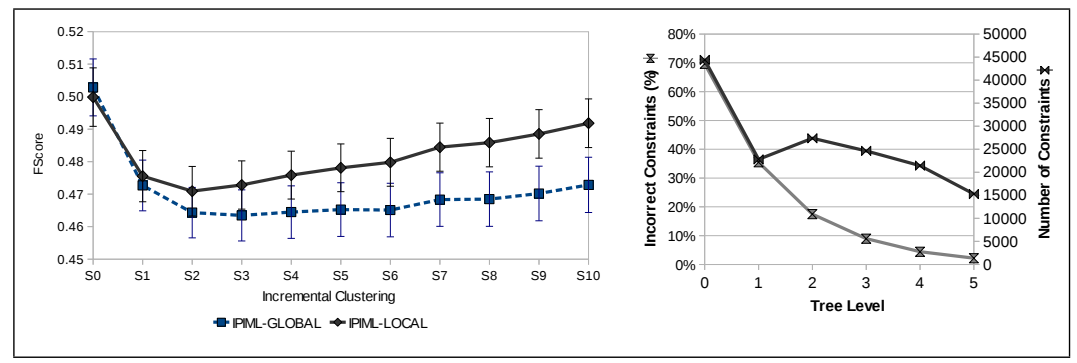

((a)) 20ng

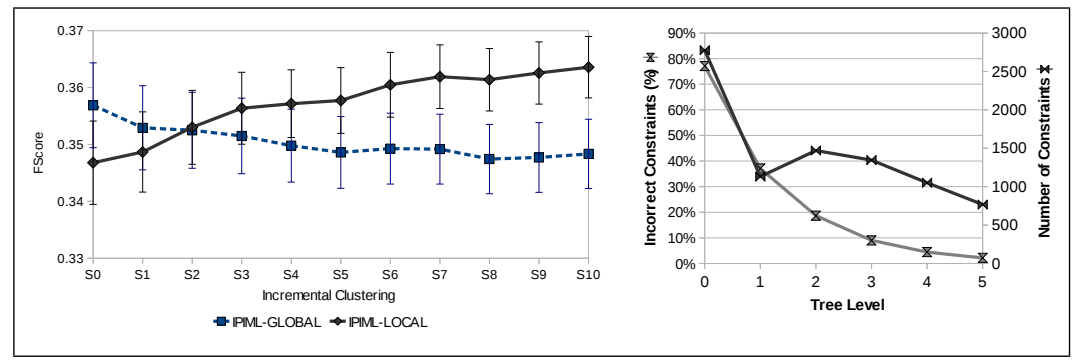

((b)) ACM

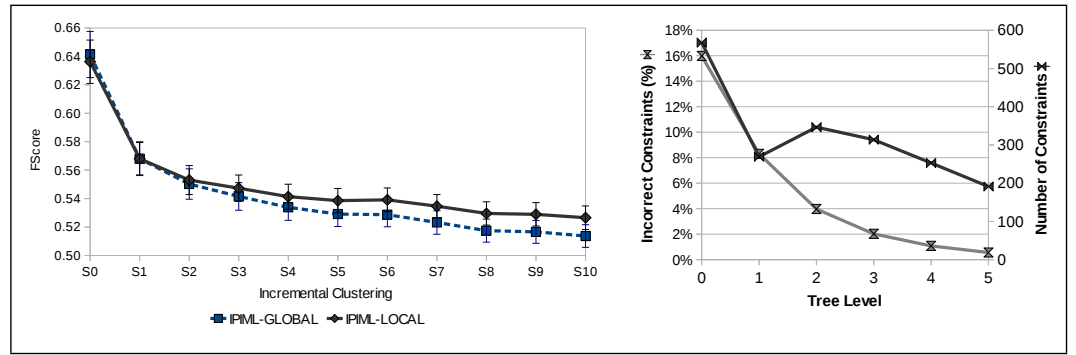

((c)) Hitech

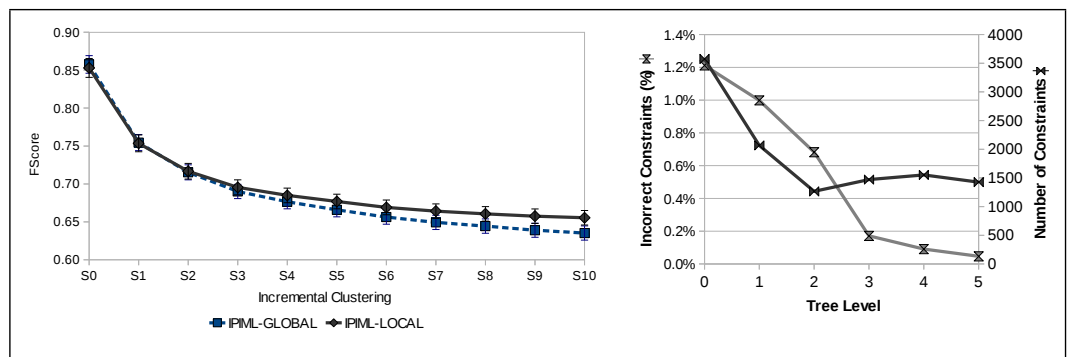

((d)) LATimes

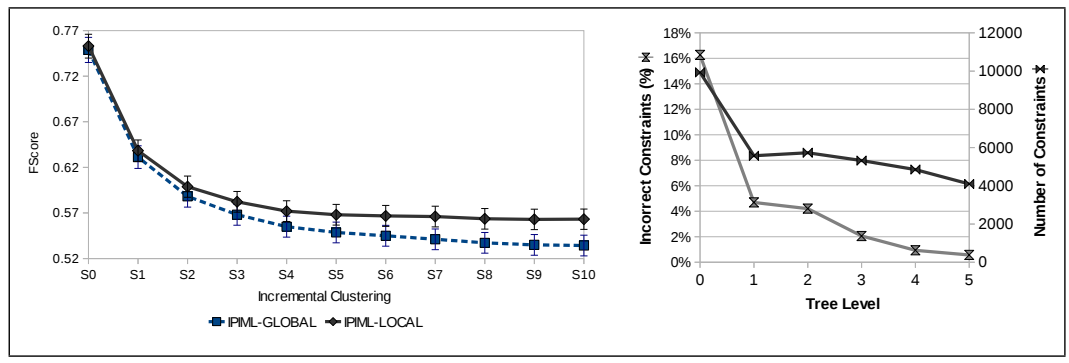

((e)) NSF

Figura 4.2: Análise comparativa da acurácia de agrupamento, no gráfico à esquerda, entre IPIML-GLOBAL e IPIML-LOCAL. No gráfico à direita são apresentados (i) a porcentagem de restrições incorretas e (ii) o número de restrições de acordo com o nivel da hierarquia. 


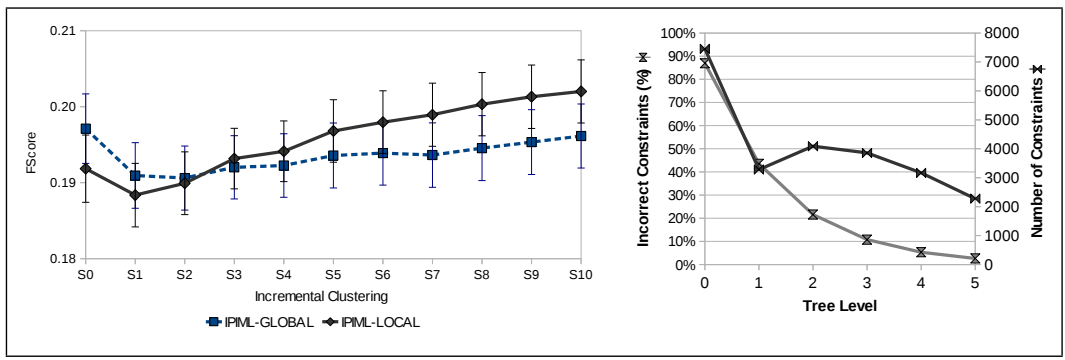

((a)) Ohsumed400

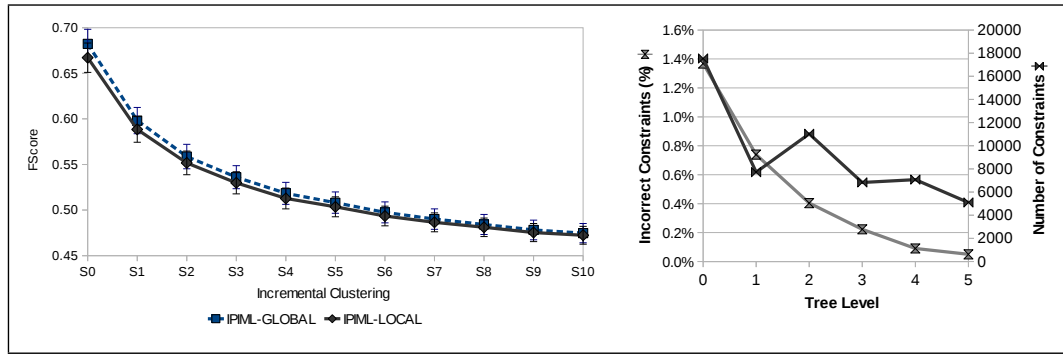

((b)) PubMed-Cancer

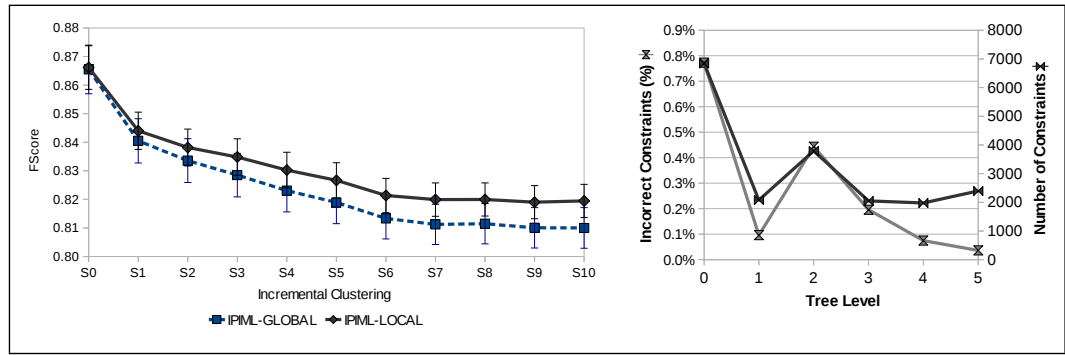

((c)) $\operatorname{Re} 8$

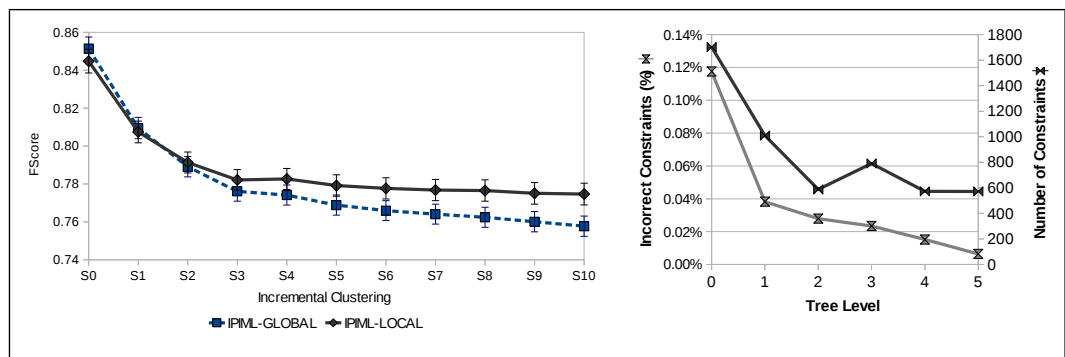

((d)) Reviews

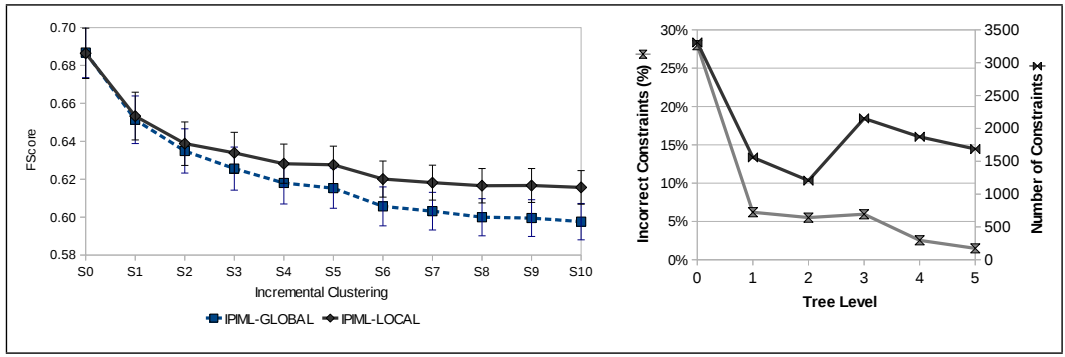

((e)) WebACE

Figura 4.3: Análise comparativa da acurácia de agrupamento, no gráfico à esquerda, entre IPIML-GLOBAL e IPIML-LOCAL. No gráfico à direita são apresentados (i) a porcentagem de restrições incorretas e (ii) o número de restrições de acordo com o nível da hierarquia. 
incorretas. Conforme as iterações do algoritmo de agrupamento hierárquico avança, a porcentagem de restrições inválidas diminui, o que pode ser explicado devido ao uso de subconjuntos de documentos cada vez mais coesos para extração de restrições. Também é observada uma redução do número médio de restrições extraídas por grupo conforme o avanço do agrupamento hierárquico. Porém, esta redução não é tão acentuada como a porcentagem de restrições incorretas. Na prática, pode ser afirmado que quanto maior o nível da hierarquia, melhor a qualidade das restrições extraídas do espaço de informação privilegiada. A seguir, é apresentada uma visão mais detalhada deste comportamento:

- Nas coleções textuais 20ng, ACM e Ohsumed400 há uma grande quantidade de restrições incorretas extraídas no início do processo de agrupamento hierárquico (raiz da hierarquia), obtendo-se entre $70 \%$ a $95 \%$ de restrições incorretas. Esse fato indica a dificuldade em incorporar informação privilegiada por meio do aprendizado de uma medida de proximidade global nessas coleções textuais. Conforme o processo de agrupamento hierárquico avança, a porcentagem de restrições incorretas extraídas nos subgrupos é drasticamente reduzida. O uso de medidas locais mais apropriadas forneceu uma melhoria na acurácia do agrupamento incremental, levando a abordagem IPIML-LOCAL a obter uma solução de agrupamento final superior em relação à abordagem IPIML-GLOBAL.

- Nas coleções textuais Hitech, NSF e WebACE há quantidade moderada de restrições incorretas extraídas no início do processo de agrupamento hierárquico, obtendo-se entre $15 \%$ a $30 \%$ de restrições incorretas. Embora o uso da abordagem IPIML-LOCAL também forneça melhores soluções finais, o impacto do uso de medidas locais na degradação do modelo durante o processo incremental é menor, indicando que há uma pequena diferença entre as estratégias para incorporação da informação privilegiada.

- Por fim, nas coleções textuais LATimes, PubMed-Cancer, Re8 e Reviews, há uma pequena quantidade de restrições incorretas extraídas no início do processo de agrupamento hierárquico, obtendo-se entre $0,1 \%$ a $1,5 \%$ de restrições inválidas. Nessas coleções textuais, o uso de uma medida global obtém resultados satisfatórios no processo de agrupamento incremental, não apresentando grande diferença de acurácia do modelo em relação ao uso de medidas de proximidade locais. 
Considerando as informações obtidas da análise experimental descritas acima, o uso da abordagem IPIML-LOCAL se mostrou promissor em cenários onde há espaço para refinar (ou corrigir) o processo de aprendizado de métricas. Como no mundo real as coleções textuais geralmente não estão rotuladas e, assim, não é possível aferir a quantidade de restrições inválidas extraídas, a abordagem IPIML-LOCAL é uma escolha mais adequada, pois apresenta resultados sempre competitivos com abordagem IPIML-GLOBAL mesmo em cenários onde o uso de uma medida global é suficiente para incorporar informação privilegiada. Além disso, embora o aprendizado de várias medidas locais requisite mais tempo computacional, este custo não afeta de forma significativa o custo total do processo de agrupamento, uma vez que o "tamanho do problema" para aprendizado da medida local é reduzido a cada nível da hierarquia (um efeito desejável na estratégia de agrupamento baseada no bisecting $k$-means).

O segundo aspecto analisado nesta avaliação experimental é sobre o quanto o uso da informação privilegiada em aprendizado de métricas melhora o processo de agrupamento quando comparado a uma estratégia Baseline. Neste caso, o Baseline representa o processo de agrupamento hierárquico tradicional baseado apenas na informação técnica. Na Figura 4.4 é apresentado um gráfico radar com a porcentagem de melhora obtido por cada abordagem em relação ao Baseline.

Para verificar se o uso de informação privilegiada em aprendizado de métricas leva a soluções de agrupamento significativamente superiores ao Baseline, foi realizada uma análise estatística com o teste não paramétrico de Friedman e pós-teste de Nemenyi (com $95 \%$ de confiança), conforme recomendações de Demšar (2006) e Japkowicz e Shah (2011). Uma representação gráfica dos resultados da análise estatística está ilustrada na Figura 4.5. Nesta representação, as abordagens estão ordenadas de acordo com o ranking calculado considerando os valores do índice $F_{S C O R E}$, dentre as diversas execuções realizadas. Uma abordagem é considerada estatisticamente superior (ou inferior) a outra se a diferença crítica CD (Critical Difference) entre elas é maior do que a CD de referência computada pelo teste. A análise estatística revela que ambas abordagens IPIML-GLOBAL e IPIML-LOCAL são superiores ao Baseline, fornecendo evidências de que a informação privilegiada foi incorporada e contribuiu positivamente na qualidade do modelo de agrupamento. Ainda, a abordagem IPIML-LOCAL obtém resultados superiores à IPIML-GLOBAL, porém as abordagens apresentam pequena diferença crítica (CD). 


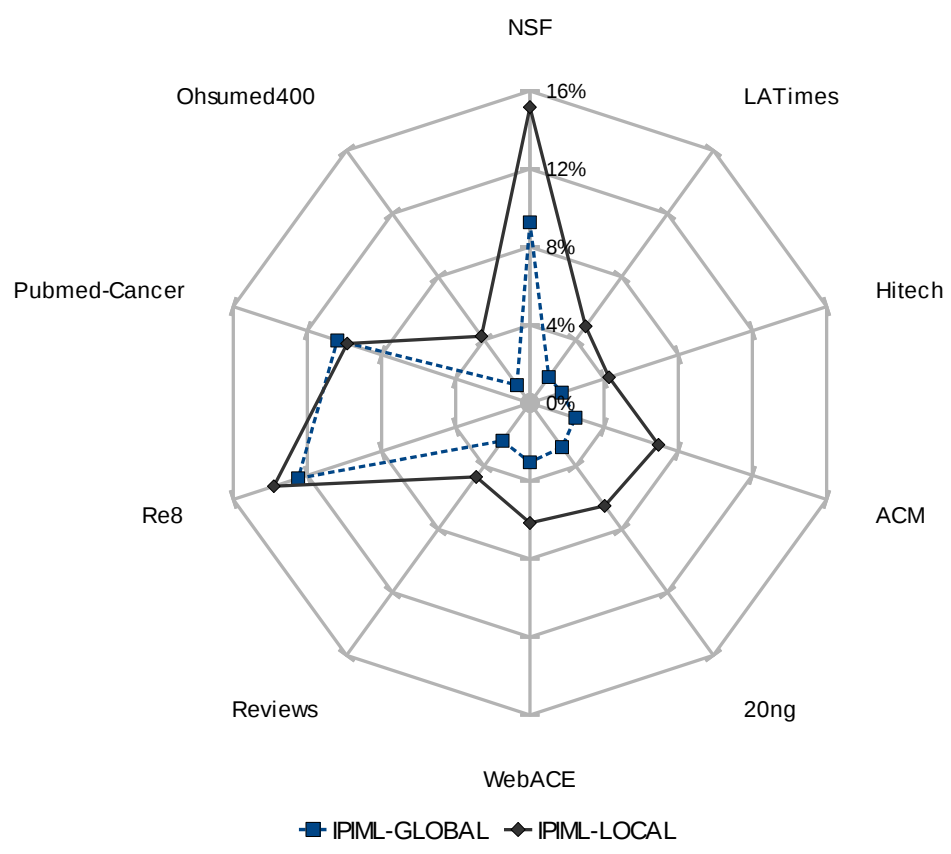

Figura 4.4: Porcentagem de melhora da solução de agrupamento com uso das abordagens IPIML-GLOBAL e IPIML-LOCAL em relação ao Baseline.

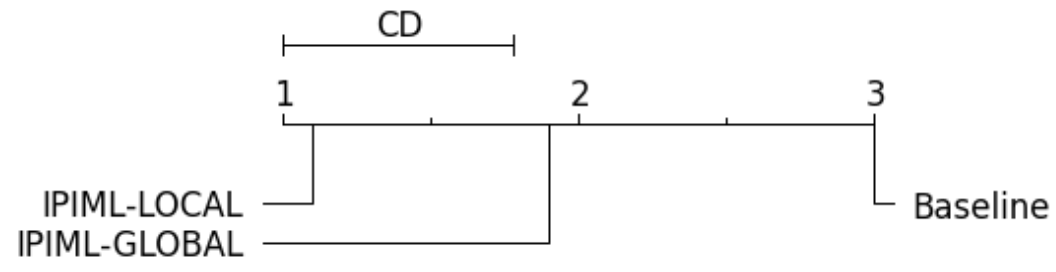

Figura 4.5: Diferença crítica $(C D)$ sobre o ranking das abordagens exploradas de acordo com a acurácia $\left(F_{S C O R E}\right)$ da solução final de agrupamento.

\subsection{Considerações Finais}

Neste capítulo foi introduzido o uso de informação privilegiada para aprendizado de métricas e aplicação em tarefas de agrupamento hierárquico. Além de adaptar a abordagem IPIML proposta por Vapnik e Vashist (2009) que obtém uma medida de proximidade global, também foi proposto o aprendizado de medidas locais para cada subgrupo do agrupamento hierárquico. Uma das principais vantagens em incorporar informação privilegiada por meio de aprendizado de métricas é que as medidas obtidas podem ser empregadas também durante o agrupamento incremental. Assim, a informação privilegiada é usada durante toda a fase do agrupamento.

Ao contrário das abordagens tradicionais de aprendizado de métricas, que 
consideram a existência prévia de uma conjunto de restrições, as abordagens aqui apresentadas dependem da extração dessas restrições a partir da informação privilegiada. O procedimento de extração não é isento a erros, uma vez que a informação privilegiada não é perfeita. Nesse sentido, os resultados experimentais fornecem evidências de que a abordagem IPIML-LOCAL é mais adequada devido à capacidade de corrigir possiveis erros do processo de extração de restrições. Como em cenários do mundo real envolvendo tarefas de agrupamento não é possivel identificar diretamente se uma restrição é inválida (já que não há dados rotulados), o uso da abordagem IPIML-LOCAL seria uma escolha mais segura do que a abordagem IPIML-GLOBAL - que obtém bons resultados apenas em cenários nos quais há pouca restrições inválidas.

Uma limitação da abordagem IPIML-LOCAL é a necessidade de adaptar o algoritmo de agrupamento hierárquico para redefinir a medida em cada iteração. Já a abordagem IPML-GLOBAL permite que a medida de proximidade seja aprendida antes do processo de agrupamento hierárquico, facilitando seu emprego em um grande gama de algoritmos existentes. 
5

\section{Informação Privilegiada para Seleção de Modelos}

\subsection{Considerações Iniciais}

A tarefa de seleção de modelos em aprendizado de máquina consiste em escolher o modelo mais adequado a partir de um conjunto de modelos (Mohri et al., 2012). Em aprendizado supervisionado, um conjunto de dados rotulados é utilizado em um procedimento de validação cruzada para determinar o modelo mais adequado, neste caso, considerando sua capacidade de generalização (Kohavi, 1995; Tan et al., 2005). Em análise de agrupamentos, a formulação de um critério para seleção de modelos é um grande desafio, principalmente devido à grande dificuldade em se definir a noção de "adequado", uma vez que geralmente não há dados rotulados disponíveis (Vaithyanathan e Dom, 1999; Xu e Wunsch, 2008; Aggarwal e Reddy, 2013).

Uma estratégia para verificar se um modelo de agrupamento é adequado é calcular o quão bem os documentos estão bem alocados em seus grupos conforme uma medida de proximidade. Por exemplo, dentre vários modelos de agrupamento, é selecionado o modelo que apresenta um baixo valor da distância intragrupo (distância entre documentos de um mesmo grupo) e alto valor para distância intergrupos (distância entre documentos de grupos diferentes) (Jain et al., 1999). Durante as últimas décadas foram propostos e investigados dezenas de índices estatísticos baseadas nas distâncias intragrupo e intergrupo para calcular o quão adequado é um modelo de agrupamento (Milligan e Cooper, 1985; Halkidi et al., 2001; Everitt et al., 2001; Faceli et al., 2005; Vendramin et al., 2010). No entanto, na maioria destes índices há o pressuposto de que a medida de distância escolhida é apropriada para 
os dados. Além disso, cada índice possui um viés que o leva a selecionar estruturas de agrupamento com determinados formatos, bem como priorizar soluções com um número maior ou menor de grupos (Vendramin et al., 2010). Assim, mesmo que do ponto de vista "estatístico" o modelo de agrupamento seja adequado, tal modelo pode não ser adequado para a aplicação ou para os usuários.

Uma outra estratégia para seleção de modelos de agrupamento depende da existência de uma organização de referência (Milligan e Cooper, 1986; Everitt et al., 2001; Xu e Wunsch, 2008). Nesses casos, o modelo mais adequado é aquele que melhor se ajusta à organização de referência, por exemplo, com o emprego de medidas de precisão e revocação (Tan et al., 2005). Embora este critério seja comum em ambientes controlados e na academia, devido à existência de coleções textuais de benchmark, seu uso em cenários reais é muito limitado, pois geralmente não há documentos rotulados. Além disso, solicitar que o usuário (ou o especialista) organize previamente uma parcela dos documentos em grupos, na prática, é uma alternativa controversa, pois são tais grupos que tanto se almeja descobrir com os algoritmos de agrupamento.

Por outro lado, os usuários possuem boa habilidade para apoiar a identificação e extração de atributos textuais do domínio, bem como incluir comentários e marcações (tags) em uma pequena parcela de documentos (Hu et al., 2011), conforme discutido no Capítulo 3. Esta informação adicional e valiosa revela estruturas de agrupamento que não estão explícitas na informação tradicional, motivando realizar o seguinte questionamento: como a existência desta informação privilegiada pode atuar na seleção de modelos de agrupamento?

Feyereisl e Aickelin (2012) investigaram o uso da informação privilegiada em análise de agrupamentos considerando um cenário de seleção de modelos. A abordagem proposta pelos autores, denominada aRi-MAX (Maximum Adjusted Rand Index), utiliza as soluções de agrupamento provenientes do espaço de informação privilegiada como organização de referência. Assim, dos diversos modelos de agrupamento obtidos no espaço de informação técnica, é selecionado o modelo que obtém melhor concordância com a organização de referência. Esta concordância é calculada por meio do índice Rand Ajustado (Hubert e Arabie, 1985) (descrito em mais detalhes na próxima seção). Embora a abordagem aRi-MAX tenha apresentado resultados promissores, a avaliação experimental é limitada a um pequeno conjunto de dados e envolve apenas agrupamentos não hierárquicos. Neste capítulo, é apresentada uma 
análise empírica mais profunda da abordagem aRi-MAX aplicada em dados textuais. Também são propostas e avaliadas duas novas abordagens, denominadas cdi-MAX (Maximum Cophenetic Difference Index) e edi-MAX (Maximum Edge Distance Index). Ao contrário da aRi-MAX, que analisa apenas partições extraídas de um dendrograma, as abordagens cdi-MAX e edi-MAX consideram a estrutura hierárquica do dendrograma, como o nível de união dos subgrupos e a topologia da árvore.

\subsection{Trabalhos Relacionados}

Os estudos sobre seleção de modelos para agrupamento se concetram principalmente em determinar a melhor partição do conjunto de dados (Fraley e Raftery, 1998; Mirkin, 2011). As partições, que representam modelos de agrupamento, podem ser obtidas por meio de um mesmo algoritmo com variação de parâmetros, por exemplo, o número $k$ de grupos, bem como por meio da execução de diferentes algoritmos de agrupamento. Algoritmos de agrupamento de hierárquico também são utilizados, uma vez que representam um conjunto aninhado de partições (Everitt et al., 2001).

Em geral, existem dois critérios para determinar qual o melhor modelo de um conjunto de modelos: critério relativo e critério externo (Xu e Wunsch, 2008). A seleção de modelos com uso de critério relativo busca determinar qual partição apresenta melhor ajuste a um mesmo conjunto de dados (Vendramin et al., 2010). Neste caso, é possivel medir quantitativamente qual o melhor número de grupos, bem como outros parâmetros dos algoritmos que geraram os modelos de agrupamento. Por outro lado, a seleção de modelos de agrupamento com uso de critério externo utiliza uma estrutura predeterminada, isto é, uma organização de referência que reflete a estrutura de agrupamento presente nos dados (Xu e Wunsch, 2008). Neste caso, será selecionado o modelo de agrupamento que apresenta maior grau de correspondência entre os grupos e a organização de referência.

A seguir, serão apresentados os trabalhos na literatura com os principais índices utilizados em cada critério, bem como discutido uma estratégia para seleção de modelos baseado na estabilidade desses índices. Também é apresentada a abordagem aRi-MAX, um dos trabalhos mais relacionados ao uso de informação privilegiada para seleção de modelos. 


\subsubsection{Critério Relativo}

Na literatura são encontradas dezenas de índices usados como critério relativo para avaliação de partições de dados (Vendramin et al., 2010). Considerando os estudos sobre validade de agrupamentos, os mais populares são o índice Davies-Bouldin (Davies e Bouldin, 1979), o índice Calinsk-Harabasz (Calinski e Harabasz, 1974), o índice de Dunn's (Dunn, 1974) e o índice Silhouette (Rousseeuw, 1987). Devido a esta grande variedade de índices para critério relativo, a escolha do índice correto é uma tarefa tão complexa quanto a escolha de um algoritmo de agrupamento. Desse modo, alguns trabalhos foram reportados comparando-se a eficácia desses índices em determinar a “qualidade” de uma estrutura de agrupamento. Milligan e Cooper (1985) compararam trinta índices visando selecionar modelos provenientes de quatro algoritmos de agrupamento hierárquico. No estudo apresentado, cada modelo representava uma partição extraída do dendrograma, o que permitia analisar qual o melhor número de grupos. Por outro lado, de acordo com Halkidi et al. (2001), esse estudo foi baseado em conjuntos de dados pequenos, com cerca de 50 exemplos, e com estruturas de agrupamento bem definidas, o que não reflete a realidade de muitas aplicações. Um estudo mais robusto sobre índices para critério relativo foi realizado por Vendramin et al. (2010), em que foram comparados cerca de 40 índices em 1080 conjuntos de dados de diferentes tamanhos e características. Entre os índices analisados, vale destacar o Silhouette que apresentou boa eficácia em diversos cenários.

O índice Silhouette verifica o quão bem os exemplos estão situados dentro de seus grupos. Dado um conjunto com $n$ exemplos $X=\left\{\vec{x}_{1}, \vec{x}_{2}, \ldots, \vec{x}_{n}\right\}$, o valor do índice $s\left(x_{i}\right)$ para o exemplo $x_{i}$ é obtido pela Equação 5.1.

$$
s\left(x_{i}\right)=\frac{b\left(\vec{x}_{i}\right)-a\left(\vec{x}_{i}\right)}{\max \left\{a\left(\vec{x}_{i}\right), b\left(\vec{x}_{i}\right)\right\}}
$$

no qual, $a\left(\vec{x}_{i}\right)$ é a distância média entre $\vec{x}_{i}$ e todos os exemplos de seu grupo; e $b\left(\vec{x}_{i}\right)$ a distância média entre $\vec{x}_{i}$ e todos os exemplos do seu grupo vizinho (o segundo grupo mais próximo).

O valor do índice Silhouette fica no intervalo $[-1,1]$, na qual valores próximos de 1 indicam que o exemplo está bem alocado no seu grupo. Com os valores de Silhouette de cada exemplo, calcula-se o valor global $\left(S_{P}\right)$ do índice para uma determinada partição, conforme ilustrado na Equação 5.2.

$$
S_{P}=\frac{\sum_{i=1}^{n} s\left(\vec{x}_{i}\right)}{n}
$$


O índice Silhouette permite comparar partições obtidas por diferentes algoritmos de agrupamento e diferentes números de grupos e, assim, a tarefa de seleção de modelos consiste em selecionar a partição com maior valor $S_{P}$. A complexidade de tempo para computar o índice é $O\left(n^{2}\right)$. Uma versão simplificada do índice Silhouette, baseada em centroides, proporciona resultados competitivos com custo computacional reduzido para $O(n)$ (Vendramin et al., 2010).

\subsubsection{Critério Externo}

Assim como os índices para critério relativo, também exitem vários índices para critério externo propostos na literatura (Faceli et al., 2005; Xu e Wunsch, 2008). No trabalho de Milligan e Cooper (1986) foram avaliados cinco índices que comparam a compatilidade entre uma partição e uma organização de referência: índice Rand (Rand, 1971), coeficiente de Jaccard (Milligan e Schilling, 1985), índice Fowlkes-Mallows (Fowlkes e Mallows, 1983) e a versões ajustadas do índice Rand propostas por Fowlkes e Mallows (1983) e Hubert e Arabie (1985). O índice Rand ajustado de Hubert e Arabie (1985) obteve os melhores resultados para seleção de modelos com critério externo e, desde então, tem sido empregado com frequência em muitos trabalhos relacionados à avaliação de estruturas de agrupamento como critério externo (Mirkin, 2012).

Para calcular o índice Rand ajustado, inicialmente é interessante discutir o cálculo do índice Rand original. Considere que $\left(\vec{x}_{i}, \vec{x}_{j}\right)$ é um par de exemplos do conjunto de dados, $P_{C}$ é a partição proveniente de um algoritmo de agrupamento, e $P_{R}$ é uma organização de referência. Ainda, considere os seguintes casos para atribuir um par de exemplos $\left(\vec{x}_{i}, \vec{x}_{j}\right)$ :

- a: $\vec{x}_{i}$ e $\vec{x}_{j}$ estão no mesmo grupo em $P_{C}$ e na mesma categoria em $P_{R}$;

- b: $\vec{x}_{i}$ e $\vec{x}_{j}$ estão no mesmo grupo em $P_{C}$ e em categorias diferentes em $P_{R}$;

- c: $\vec{x}_{i}$ e $\vec{x}_{j}$ estão em grupos diferentes em $P_{C}$ e na mesma categoria em $P_{R}$; $\mathrm{e}$

- d: $\vec{x}_{i}$ e $\vec{x}_{j}$ estão em grupos diferentes em $P_{C}$ e em categorias diferentes em $P_{R}$.

O cálculo do índice Rand está expresso na Equação 5.3, utilizando as contagens dos casos acima. $O$ índice possui valores no intervalo $[-1,1]$, e assume 
valor próximo de 1 conforme o resultado do agrupamento se aproxima da partição de referência.

$$
R=\frac{a+d}{a+b+c+d}
$$

Uma limitação bem conhecida do índice Rand é o favorecimento de partições com maior número de grupos, uma vez que o caso d tende a dominar o valor do índice (Hubert e Arabie, 1985). Isso torna o índice pouco sensível em detectar partições aleatórias, ou seja, o valor do índice não é nulo ao comparar duas partições aleatórias dos dados. Nesse sentido, o índice Rand ajustado proposto por Hubert e Arabie (1985) elimina essas limitações. O índice Rand ajustado está definido na Equação 5.4.

$$
R_{\text {ajustado }}=\frac{a-\frac{(a+c)(a+b)}{(a+b+c+d)}}{\frac{(a+c)+(a+b)}{2}-\frac{(a+c)(a+b)}{(a+b+c+d)}}
$$

\subsubsection{Estabilidade do Modelo}

Em aprendizado supervisionado, o uso de validação cruzada é uma das principais técnicas para seleção de modelos (Mohri et al., 2012). De modo geral, a validação cruzada consiste em empregar um subconjunto dos dados para treinamento do modelo e utilizar o restante dos dados como um conjunto de teste para estimar a acurácia do modelo em dados ainda não vistos (Tan et al., 2005; Japkowicz e Shah, 2011). Embora esta técnica seja potencialmente útil para seleção de modelos de classificação e regressão, seu uso não é diretamente aplicável para modelos de agrupamento devido a ausência de conjuntos de dados rotulados (Mirkin, 2012).

Lange et al. (2002) propuseram uma estratégia alternativa de validação cruzada para seleção de modelos de agrupamento, introduzindo o conceito de estabilidade do modelo (Lange et al., 2004). A ideia intuitiva desta estratégia é que se for realizada repetidas reamostragem dos dados e execução do algoritmo de agrupamento nessas amostras, então um bom algoritmo de agrupamento será aquele não obtém modelos muito variados nas diversas execuções. Em outras palavras, o modelo obtido deve ser estável em relação às diversas subamostras dos dados. Esta estratégia também é utilizada em trabalhos sobre validação de agrupamento por "capacidade de predição" (Tibshirani e Walther, 2005; Volkovich et al., 2008). Embora existam diferenças pontuais entre esses trabalhos, a ideia básica da estratégia de validação cruzada para seleção de modelos possui os seguintes passos: 
1. Dividir aleatoriamente o conjunto de dados em dois subconjuntos, um para treino $X_{R}$ e outro para teste $X_{S}$;

2. A partir de um mesmo algoritmo de agrupamento $H$, obter um modelo de agrupamento $M_{R}$ a partir de $X_{R}$ e um modelo de agrupamento $M_{S}$ a partir de $X_{S}$;

3. Utilizar a estrutura de agrupamento representada no modelo $M_{R}$ para "classificar" os exemplos do subconjunto de teste $X_{S}$, obtendo-se o modelo de agrupamento $M_{S}^{\prime}$;

4. Comparar a compatibilidade entre os modelos $M_{S}$ e $M_{S}^{\prime}$ a partir de uma estatística $\gamma$; e

5. Repetir os passos 1-4 um número $b$ de vezes para estimar a estabilidade do modelo por meio da média dos valores $\gamma$.

Os parâmetros $H$ (algoritmo de agrupamento), $\gamma$ (estatística) e $b$ devem ser definidos pelo usuário. O algoritmo de agrupamento $H$ é responsável por obter o modelo em que se deseja analisar. A estatística $\gamma$, na prática, é um índice de validação para critério externo, como o índice Rand ajustado, que compara duas estruturas de agrupamento. O número de vezes que os passos são executados (b) permite calcular a média e desvio padrão do índice, que irá refletir a estabilidade do modelo. De acordo com Lange et al. (2002), o mesmo procedimento pode ser repetido com diferentes algoritmos de agrupamento e/ou diferentes números de grupos para seleção de modelos (observando-se as mesmas divisões dos conjuntos de treino e teste).

Um ponto importante na análise da estabilidade do modelo é a estratégia de "classificação" utilizada no passo 3. O modelo de agrupamento $M_{S}^{\prime}$ é obtido alocando-se os exemplos do subconjunto $X_{S}$ a um dos grupos do modelo $M_{R}$ por meio da menor distância entre exemplos e centros dos grupos. A medida de distância utilizada deve ser a mesma definida no algoritmo de agrupamento $H$.

É importante observar que a análise de estabilidade do modelo é uma estratégia natural em abordagens de agrupamento no paradigma LUPI. O aprendizado de um modelo inicial é realizado a partir de uma amostra de "treino", considerando-se os documentos que possuem informação técnica e informação privilegiada. O modelo inicial é então utilizado para agrupamento incremental dos restantes dos documentos, que é associado a etapa de teste do modelo. Nesse ponto de vista, espera-se que a informação privilegiada seja 
útil para obter um modelo de inicial de maior qualidade e, consequentemente, melhorar a estabilidade do modelo final.

\subsubsection{A Abordagem aRi-MAX}

A abordagem aRi-MAX utiliza os conceitos de seleção de modelos para agrupamento de dados com uso de informação privilegiada (Feyereisl e Aickelin, 2012). A ideia geral do aRi-MAX é selecionar, dentro um conjunto de modelos, o modelo que obtém maior concordância nos dois espaços de características $\chi$ (informação técnica) e $\chi^{*}$ (informação privilegiada).

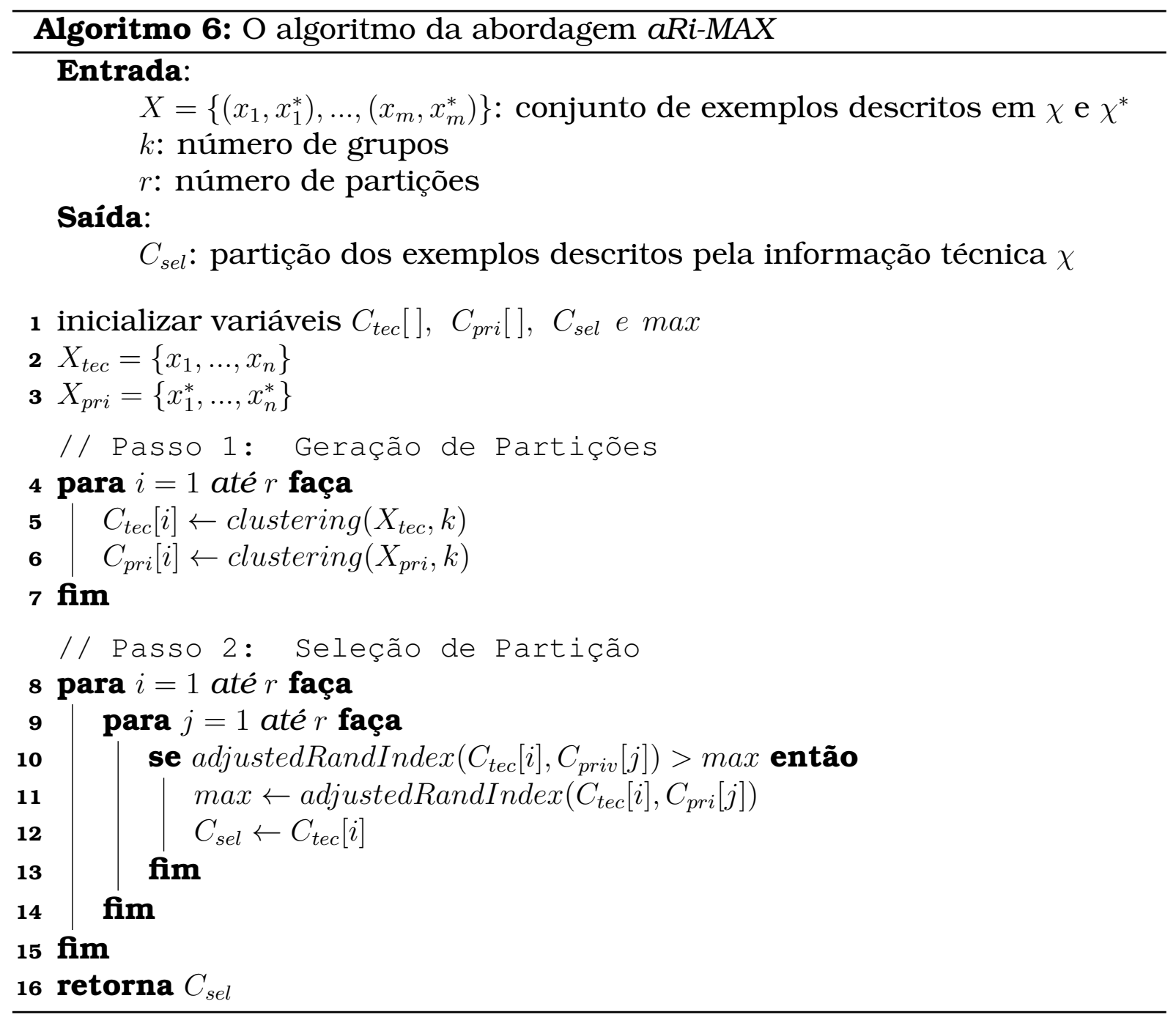

Os detalhes da abordagem aRi-MAX estão ilustrados no Algoritmo 6, que é dividido em dois passos: (1) Geração de Partições e (2) Seleção de Partição. Considere um conjunto com $m$ exemplos $X=\left\{\left(x_{1}, x_{1}^{*}\right), \ldots,\left(x_{m}, x_{m}^{*}\right)\right\}, x_{i} \in \chi, x_{i}^{*} \in$ $\chi^{*}$. Inicialmente, nas linhas $2-3$, os exemplos do conjunto $X$ são reorganizados 
em dois conjuntos $X_{t e c}$ e $X_{p r i}$, ou seja, conjuntos com exemplos descritos pela informação técnica e informação privilegiada, respectivamente. Em seguida, é realizado o processo de geração de partições. Para tal, são obtidas $r$ partições tanto para o conjunto $X_{t e c}$ quanto para o conjunto $X_{p r i}$, conforme descrito nas linhas $4-7$. A função clustering () representa algoritmos de agrupamento particional, por exemplo, o $k$-means. É importante observar que, em cada iteração, um algoritmo de agrupamento diferente pode ser executado (ou um mesmo algoritmo com diferentes parâmetros e/ou inicializações) para fornecer diversidade entre as partições obtidas. As variáveis $C_{t e c}$ [] e $C_{p r i}$ [] apontam para uma lista de $r$ partições dos conjuntos $X_{t e c}$ e $X_{p r i}$, respectivamente.

Para o passo de seleção de partição, cada partição obtida dos exemplos de informação técnica é comparada às partições obtidas dos exemplos de informação privilegiada. Esta comparação busca verificar o grau de correspondência entre os dois agrupamentos por meio do índice Rand ajustado. A partição de $X_{t e c}$ que obtém o maior valor de índice Rand a uma partição de $X_{p r i}$ é selecionada pelo aRi-MAX, conforme descrito nas linhas $10-13$.

$\mathrm{Na}$ abordagem aRi-MAX, a informação privilegiada é incorporada de forma indireta, pois atua como um guia para selecionar uma das possíveis partições provenientes da informação técnica.

\subsection{Abordagens propostas: cdi-MAX e edi-MAX}

Neste trabalho são propostas duas abordagens que, como a aRi-MAX, utilizam a informação privilegiada para seleção de modelos de agrupamento hierárquico. A abordagem aRi-MAX analisa partições para seleção de modelos com uso do índice Rand ajustado. Embora seja possível seu uso em modelos de agrupamento hierárquico, por meio da extração de partições do dendrograma, algumas informações importantes sobre a estrutura hierárquica não são consideradas durante a seleção de modelos. Nesse sentido, as abordagens propostas cdi-MAX e edi-MAX empregam índices específicos para comparação de estruturas hierárquicas. As diferenças entre essas abordagens também podem ser expressadas pelas medidas de correlação utilizadas. Na abordagem cdi-MAX, é utilizada a correlação de Pearson que permite identificar a dependência linear entre duas variávies, no caso, as matrizes de descritores. Ainda, há o pressuposto de que as variáveis possuam distribuição normal. Já na abordagem edi-MAX, é utilizada a correlação de Spearman que calcula a dependência entre duas variáveis considerando a relação de ordem dos seus 
valores (ranking). Nesse caso, não é necessário definir previamente a distribuição dessas variáveis (Campello e Hruschka, 2009).

A comparação de estruturas hierárquicas, como dendrogramas, é baseada nas chamadas matrizes de descritores (Podani, 2000; Zheng et al., 2010). Uma matriz de descritores é análoga a uma matriz de distâncias entre exemplos. No entanto, a proximidade entre dois exemplos é definida pelo valor de um descritor extraído do dendrograma. Dessa forma, a matriz de descritores é uma forma de reconstituir a matriz de distâncias a partir do dendrograma e, assim, também permite representar o resultado do agrupamento hierárquico (Mirzaei et al., 2008).

Vários descritores foram propostos considerando os diferentes aspectos de um dendrograma (Podani, 2000). Em geral, um descritor deve expressar a posição relativa entre um par de exemplos no dendrograma. Esta posição relativa pode considerar basicamente dois tipos de descritores. O primeiro é baseado no valor da distância da união entre dois subgrupos, ou seja, o valor real (eixo y) no dendrograma que indica a fusão de dois subgrupos (o valor desta altura é dependente do algoritmo de agrupamento hierárquico utilizado). O segundo considera o nível da fusão de dois exemplos no dendrograma. Na literatura é comum denominar o primeiro tipo como Weighted Dendrogram Descriptors e o segundo tipo como Ranked Dendrogram Descriptors (Podani, 2000).

A abordagem proposta cdi-MAX utiliza o descritor Cophenetic Difference que pertence ao primeiro tipo, enquanto a abordagem edi-MAX utiliza o descritor Edge Distance pertencente ao segundo tipo. Para ilustrar melhor a diferença entre esses descritores, considere o esquema apresentado na Figura 5.1. O dendrograma do exemplo foi obtido após a execução do algoritmo de agrupamento hierárquico Single-Linkage a partir da matriz de distâncias entre oito exemplos $(a, b, c, d, e, f, g$ e $h)$. As matrizes referentes a cada descritor também estão apresentadas na Figura 5.1 e serão utilizadas para exemplificar as abordagens descritas nas próximas seções.

\subsubsection{Cophenetic Difference Index (cdi)}

O descritor Cophenetic Difference $(C D)$ define a proximidade relativa entre dois exemplos $i$ e $j$, a partir de um dendrograma $H$, como o valor da altura (eixo y) do primeiro subgrupo a unir os exemplos $i$ e $j$ em $H$. Por exemplo, o valor do descritor $C D$ para os exemplos $a$ e $b$ no dendrograma $H$ é $C D_{H}(a, b)=0,1$, conforme pode ser visualizado no dendrograma da Figura 5.1. Já os exemplos 


\begin{tabular}{|c|c|c|c|c|c|c|c|c|} 
& $\mathbf{a}$ & $\mathbf{b}$ & $\mathbf{c}$ & $\mathbf{d}$ & $\mathbf{e}$ & $\mathbf{f}$ & $\mathbf{g}$ & $\mathbf{h}$ \\
\hline $\mathbf{a}$ & $\mathbf{0}$ & $\mathbf{0 , 1}$ & $\mathbf{0 , 3 5}$ & $\mathbf{0 , 7 5}$ & $\mathbf{0 , 7}$ & $\mathbf{0 , 6 5}$ & $\mathbf{0 , 9}$ & $\mathbf{1}$ \\
\hline $\mathbf{b}$ & $\mathbf{0 , 1}$ & $\mathbf{0}$ & $\mathbf{0 , 8}$ & $\mathbf{0 , 9 5}$ & $\mathbf{0 , 8 5}$ & $\mathbf{0 , 7}$ & $\mathbf{0 , 6}$ & $\mathbf{0 , 9 5}$ \\
\hline c & $\mathbf{0 , 3 5}$ & $\mathbf{0 , 8}$ & $\mathbf{0}$ & $\mathbf{0 , 4}$ & $\mathbf{0 , 7}$ & $\mathbf{1}$ & $\mathbf{0 , 8}$ & $\mathbf{0 , 7 5}$ \\
\hline d & $\mathbf{0 , 7 5}$ & $\mathbf{0 , 9 5}$ & $\mathbf{0 , 4}$ & $\mathbf{0}$ & $\mathbf{0 , 1 5}$ & $\mathbf{0 , 9 5}$ & $\mathbf{0 , 8 5}$ & $\mathbf{1}$ \\
\hline e & $\mathbf{0 , 7}$ & $\mathbf{0 , 8 5}$ & $\mathbf{0 , 7}$ & $\mathbf{0 , 1 5}$ & $\mathbf{0}$ & $\mathbf{0 , 2 5}$ & $\mathbf{1}$ & $\mathbf{0 , 7}$ \\
\hline f & $\mathbf{0 , 6 5}$ & $\mathbf{0 , 7}$ & $\mathbf{1}$ & $\mathbf{0 , 9 5}$ & $\mathbf{0 , 2 5}$ & $\mathbf{0}$ & $\mathbf{0 , 8 5}$ & $\mathbf{1}$ \\
\hline g & $\mathbf{0 , 9}$ & $\mathbf{0 , 6}$ & $\mathbf{0 , 8}$ & $\mathbf{0 , 8 5}$ & $\mathbf{1}$ & $\mathbf{0 , 8 5}$ & $\mathbf{0}$ & $\mathbf{0 , 2}$ \\
\hline h & $\mathbf{1}$ & $\mathbf{0 , 9 5}$ & $\mathbf{0 , 7 5}$ & $\mathbf{1}$ & $\mathbf{0 , 7}$ & $\mathbf{1}$ & $\mathbf{0 , 2}$ & $\mathbf{0}$ \\
\hline
\end{tabular}

Matriz de Distâncias

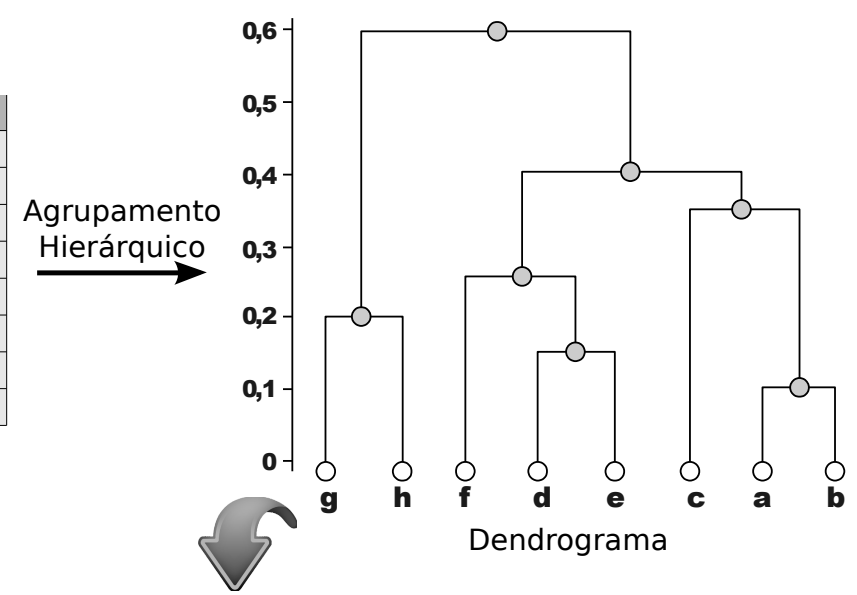

Matrizes de Descritores

\begin{tabular}{|c|c|c|c|c|c|c|c|c|} 
& $\mathbf{a}$ & $\mathbf{b}$ & $\mathbf{c}$ & $\mathbf{d}$ & $\mathbf{e}$ & $\mathbf{f}$ & $\mathbf{g}$ & $\mathbf{h}$ \\
\hline a & $\mathbf{0}$ & $\mathbf{0 , 1}$ & $\mathbf{0 , 3 5}$ & $\mathbf{0 , 4}$ & $\mathbf{0 , 4}$ & $\mathbf{0 , 4}$ & $\mathbf{0 , 6}$ & $\mathbf{0 , 6}$ \\
\hline b & $\mathbf{0 , 1}$ & $\mathbf{0}$ & $\mathbf{0 , 3 5}$ & $\mathbf{0 , 4}$ & $\mathbf{0 , 4}$ & $\mathbf{0 , 4}$ & $\mathbf{0 , 6}$ & $\mathbf{0 , 6}$ \\
\hline c & $\mathbf{0 , 3 5}$ & $\mathbf{0 , 3 5}$ & $\mathbf{0}$ & $\mathbf{0 , 4}$ & $\mathbf{0 , 4}$ & $\mathbf{0 , 4}$ & $\mathbf{0 , 6}$ & $\mathbf{0 , 6}$ \\
\hline d & $\mathbf{0 , 4}$ & $\mathbf{0 , 4}$ & $\mathbf{0 , 4}$ & $\mathbf{0}$ & $\mathbf{0 , 1 5}$ & $\mathbf{0 , 2 5}$ & $\mathbf{0 , 6}$ & $\mathbf{0 , 6}$ \\
\hline e & $\mathbf{0 , 4}$ & $\mathbf{0 , 4}$ & $\mathbf{0 , 4}$ & $\mathbf{0 , 1 5}$ & $\mathbf{0}$ & $\mathbf{0 , 2 5}$ & $\mathbf{0 , 6}$ & $\mathbf{0 , 6}$ \\
\hline f & $\mathbf{0 , 4}$ & $\mathbf{0 , 4}$ & $\mathbf{0 , 4}$ & $\mathbf{0 , 2 5}$ & $\mathbf{0 , 2 5}$ & $\mathbf{0}$ & $\mathbf{0 , 6}$ & $\mathbf{0 , 6}$ \\
\hline g & $\mathbf{0 , 6}$ & $\mathbf{0 , 6}$ & $\mathbf{0 , 6}$ & $\mathbf{0 , 6}$ & $\mathbf{0 , 6}$ & $\mathbf{0 , 6}$ & $\mathbf{0}$ & $\mathbf{0 , 2}$ \\
\hline h & $\mathbf{0 , 6}$ & $\mathbf{0 , 6}$ & $\mathbf{0 , 6}$ & $\mathbf{0 , 6}$ & $\mathbf{0 , 6}$ & $\mathbf{0 , 6}$ & $\mathbf{0 , 2}$ & $\mathbf{0}$ \\
\hline
\end{tabular}

Cophenetic Difference

\begin{tabular}{|c|c|c|c|c|c|c|c|c|} 
& a & b & c & d & e & f & g & h \\
\hline a & $\mathbf{0}$ & $\mathbf{1}$ & $\mathbf{2}$ & $\mathbf{3}$ & $\mathbf{3}$ & $\mathbf{3}$ & $\mathbf{4}$ & $\mathbf{4}$ \\
\hline $\mathbf{b}$ & $\mathbf{1}$ & $\mathbf{0}$ & $\mathbf{2}$ & $\mathbf{3}$ & $\mathbf{3}$ & $\mathbf{3}$ & $\mathbf{4}$ & $\mathbf{4}$ \\
\hline c & $\mathbf{2}$ & $\mathbf{2}$ & $\mathbf{0}$ & $\mathbf{3}$ & $\mathbf{3}$ & $\mathbf{3}$ & $\mathbf{4}$ & $\mathbf{4}$ \\
\hline d & $\mathbf{3}$ & $\mathbf{3}$ & $\mathbf{3}$ & $\mathbf{0}$ & $\mathbf{1}$ & $\mathbf{2}$ & $\mathbf{4}$ & $\mathbf{4}$ \\
\hline e & $\mathbf{3}$ & $\mathbf{3}$ & $\mathbf{3}$ & $\mathbf{1}$ & $\mathbf{0}$ & $\mathbf{2}$ & $\mathbf{4}$ & $\mathbf{4}$ \\
\hline $\mathbf{f}$ & $\mathbf{3}$ & $\mathbf{3}$ & $\mathbf{3}$ & $\mathbf{2}$ & $\mathbf{2}$ & $\mathbf{0}$ & $\mathbf{4}$ & $\mathbf{4}$ \\
\hline $\mathbf{g}$ & $\mathbf{4}$ & $\mathbf{4}$ & $\mathbf{4}$ & $\mathbf{4}$ & $\mathbf{4}$ & $\mathbf{4}$ & $\mathbf{0}$ & $\mathbf{1}$ \\
\hline $\mathbf{h}$ & $\mathbf{4}$ & $\mathbf{4}$ & $\mathbf{4}$ & $\mathbf{4}$ & $\mathbf{4}$ & $\mathbf{4}$ & $\mathbf{1}$ & $\mathbf{0}$ \\
\hline
\end{tabular}

Edge Difference

Figura 5.1: Exemplo de matrizes de descritores $C D$ e $E D$ extraídas de um dendrograma. O dendrograma foi gerado com uso do algoritmo de agrupamento hierárquico Single-Linkage a partir da referida matriz de distâncias.

a e $g$ possuem valor $C D_{H}(a, g)=0,6$, pois foram unidos apenas na raiz do dendrograma.

Neste trabalho é proposto o uso do índice cdi, definido na Equação 5.5, para a seleção de modelos com apoio de informação privilegiada. Nesta equação, $H$ é um dendrograma obtido a partir dos exemplos representados no espaço de informação técnica e $H^{*}$ é um dendrograma de referência obtido a partir do espaço de informação privilegiada. Os termos $\overline{C D}_{H}$ e $\overline{C D}_{H^{*}}$ representam, respectivamente, a média dos valores dos descritores $C D$ dos diferentes pares de exemplos $i$ e $j$ (matriz triangular).

$$
c d i\left(H, H^{*}\right)=\frac{\sum_{i<j}\left(C D_{H}(i, j)-\overline{C D}_{H}\right)\left(C D_{H^{*}}(i, j)-\overline{C D}_{H^{*}}\right)}{\sqrt{\left[\sum_{i<j}\left(C D_{H}(i, j)-\overline{C D}_{H}\right)^{2}\right]\left[\sum_{i<j}\left(C D_{H^{*}}(i, j)-\overline{C D}_{H^{*}}\right)^{2}\right]}}
$$

O índice $c d i$, na prática, computa a correlação de Pear son entre as matrizes de descritores $C D$ extraídas dos dendrogramas de informação técnica e informação privilegiada. Se os dois dendrogramas possuem um alto grau de correspondência, então o valor do índice se aproxima de 1. Caso contrário, se 
aproxima de -1 . A seleção de modelos busca, então, selecionar um dendrograma dentre um conjunto de $r$ dendrogramas $D=\left\{H_{1}, H_{2}, \ldots, H_{r}\right\}$ do espaço de informação técnica. O dendrograma selecionado é que aquele maximiza o índice $c d i$, conforme a Equação 5.6.

$$
c d i-M A X(D)=\underset{\forall H \in D}{\arg \max } c d i\left(H, H^{*}\right)
$$

\subsubsection{Edge Distance Index (edi)}

O descritor Edge Distance $(E D)$ define a proximidade relativa entre dois exemplos $i$ e $j$, a partir de um dendrograma $H$, considerando apenas topologia da hierarquia. Assim, em vez de identificar a altura (eixo y) de um subgrupo, o descritor $E D$ precisa identificar o nível $(N L)$ do subgrupo. Seja $G_{u}$ um determinado subgrupo de um dendrograma $H$. Se $G_{u}$ for um grupo unitário, ou seja, um nó folha da árvore, então o subgrupo está no nível zero $\left(N L\left(G_{u}\right)=0\right)$. Caso contrário, o nível do subgrupo $G_{u}$ é definido como o valor $N L$ do seu subgrupo filho com maior nível acrescido de um. Formalmente, os níveis podem ser calculados conforme a Equação 5.7.

$$
N L\left(G_{u}\right)= \begin{cases}\operatorname{MAX}\left(N L\left(G_{v}\right), N L\left(G_{w}\right)\right)+1 & \text { se } G_{u}=G_{v} \cup G_{w} \\ 0 & \text { se } G_{u} \text { contém apenas um exemplo }\end{cases}
$$

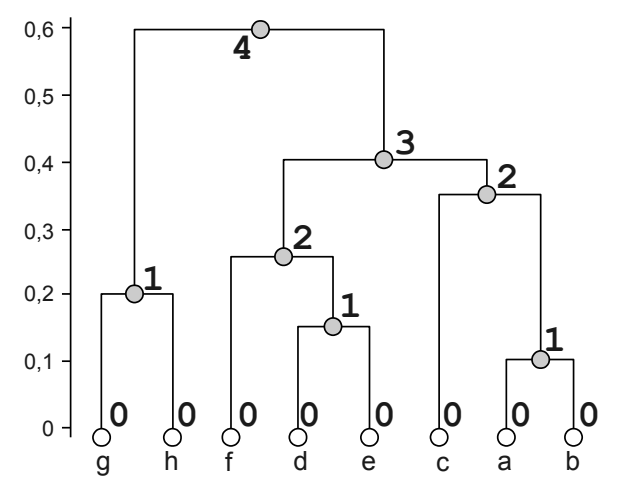

Figura 5.2: Dendrograma com identificação de nível $(N L)$ para cada subgrupo.

Para ilustrar a identificação dos níveis de cada subgrupo, na Figura 5.2 é reapresentado o dendrograma do exemplo anterior com seus respectivos níveis.

O valor do descritor $E D$ entre dois exemplos $i$ e $j$ é definido, então, como o valor do nível do primeiro subgrupo a unir os exemplos $i$ e $j$. Dessa forma, 
conforme pode ser observado nos exemplos da Figura 5.1 e 5.2, os exemplos a e $b$ possuem $E D_{H}(a, b)=1$. Já os exemplos $a$ e $g$, que foram unidos na raiz do dendrograma $H$, possuem valor $E D_{H}(a, g)=4$.

O uso do índice edi (Equação 5.8) é proposto neste trabalho para seleção de modelos com apoio de informação privilegiada. Seu funcionamento é similar ao índice $c d i$. No entanto, a correlação é computada com base no ranking dos descritores $E D$, ou seja, é utilizada a correlação de Spearman para verificar o grau de correspondência entre o dendrograma de informação técnica $H$ e o dendrograma de informação privilegiada $H^{*}$.

$$
e d i\left(H, H^{*}\right)=\frac{\sum_{i<j}\left(E D_{H}(i, j)-\overline{E D}_{H}\right)\left(E D_{H^{*}}(i, j)-\overline{E D}_{H^{*}}\right)}{\sqrt{\left[\sum_{i<j}\left(E D_{H}(i, j)-\overline{E D}_{H}\right)^{2}\right]\left[\sum_{i<j}\left(E D_{H^{*}}(i, j)-\overline{E D}_{H^{*}}\right)^{2}\right]}}
$$

Por fim, dentre um conjunto de $r$ dendrogramas $D=\left\{H_{1}, H_{2}, \ldots, H_{r}\right\}$ do espaço de informação técnica, é selecionado o dendrograma com maior valor do índice edi, conforme definido na Equação 5.9.

$$
e d i-M A X(D)=\underset{\forall H \in D}{\arg \max } e d i\left(H, H^{*}\right)
$$

\subsection{Aspectos Teóricos}

Ao contrário da abordagem aRi-MAX, as abordagens cdi-MAX e edi-MAX permitem utilizar informações sobre a estrutura hierárquica do dendrograma para a seleção de modelos. O fato da edi-MAX explorar apenas o nível da fusão dos grupos indica uma preferência maior pela topologia da árvore, ou seja, dois dendrogramas serão considerados bem correlacionados se possuem uma topologia similar, mesmo que os valores das distâncias de fusão dos grupos sejam discrepantes. A abordagem cdi-MAX também considera a topologia da hierarquia, mas dando preferência à distância de fusão dos grupos.

Em teoria, o uso da abordagem cdi-MAX é preferível se os valores das distâncias entre exemplos no espaço de informação privilegiada são confiáveis para a aplicação, pois será selecionado o modelo de agrupamento que irá superajustar essas distâncias. No entanto, o uso de diferentes algoritmos de agrupamento hierárquico (ou diferentes medidas de distância) pode levar a valores de distância de fusão dos grupos que não são diretamente comparáveis. Nesses casos, o uso da abordagem edi-MAX é preferível.

Uma forma de visualizar a diferença entre as abordagens é analisar a propriedade ultramétrica de cada matriz de descritores, que pode ser vista como 
a capacidade de se reconstruir o dendrograma original. Isso pode ser feito aplicando-se o algoritmo de agrupamento hierárquico Single-link a partir da matriz de descritores. Na Figura 5.3 é ilustrado os dendrogramas obtidos a partir das matrizes de descritores $C D$ e $E D$ apresentados no exemplo da Figura 5.1. Note que o descritor $C D$ permite recuperar o dendrograma original, enquanto o descritor $E D$ recupera apenas sua topologia.

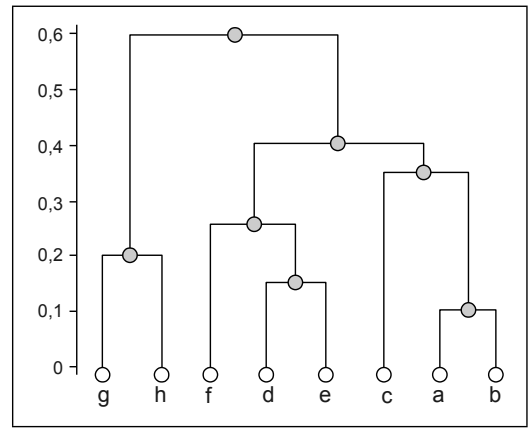

((a)) CD Dendrograma

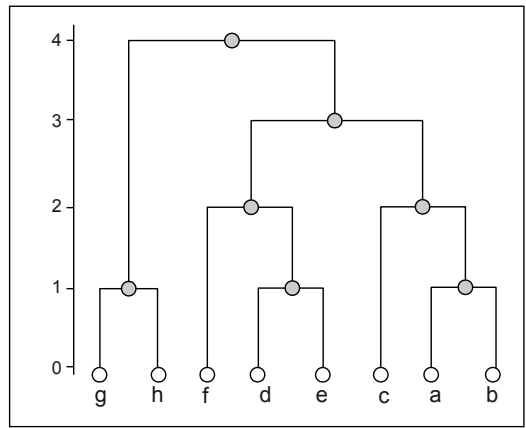

((b)) ED Dendrograma

Figura 5.3: Reconstrução do dendrograma a partir da matriz de descritores. (a) Dendrograma reconstruído a partir da matriz de descritores $C D$ é igual ao dendrograma original. (b) Dendrograma reconstruído a partir da matriz de descritores $E D$ recupera a topologia do dendrograma original.

\subsection{Análise Experimental}

A abordagem aRi-MAX (Feyereisl e Aickelin, 2012) e as abordagens propostas neste trabalho, cdi-MAX e edi-MAX, são comparadas empiricamente visando avaliar a eficácia de cada uma em empregar informação privilegiada para seleção de modelos.

$\mathrm{Na}$ análise experimental foram utilizadas as 10 coleções textuais de benchmark descritas no Capítulo 3. Para estimar a acurácia de cada abordagem, foi utilizado o índice $F_{S C O R E}$ em um processo de validação cruzada para agrupamento. O subconjunto $S_{0}$ que contém documentos textuais representados em ambos espaços de informação técnica e privilegiada é utilizado para aprendizado do modelo inicial, por meio das abordagens aRi-MAX, cdi-MAX ou edi-MAX. Após a obtenção do modelo inicial, o restante dos documentos (representados apenas pela informação técnica) são incorporados ao modelo por meio de agrupamento incremental. Para aferir a estabilidade do modelo inicial, o processo de agrupamento incremental é avaliado em dez subconjuntos (balanceados) de documentos, $S_{1}, S_{2}, \ldots, S_{10}$. Este processo de avaliação 
experimental é repetido com 250 diferentes amostragem dos dados e são apresentados as respectivas médias e desvios padrão do índice $F_{S C O R E}$. Os detalhes da configuração experimental são descritos abaixo.

- Em cada abordagem, durante a etapa de aprendizado do modelo inicial, foram obtidos 30 diferentes dendrogramas a partir dos documentos com informação técnica do subconjunto $S_{0}$. Um dendrograma de referência é obtido a partir dos documentos com informação privilegiada do subconjunto $S_{0}$. Para obtenção dos dendrogramas foi utilizado o algoritmo de agrupamento hierárquico Bisecting $k$-means com similaridade cosseno e 10 inicializações dos centroides.

- Um mesmo dendrograma de referência é utilizado para a seleção de modelos de cada abordagem. Dessa forma, é possível verificar qual índice selecionou o melhor modelo de agrupamento (dendrograma) do espaço de informação técnica. No caso da abordagem aRi-MAX, foi extraído uma partição do dendrograma de referência utilizado o índice Silhouette, testando-se valores de $k$ no intervalo de 2 até $\sqrt{\left|S_{0}\right|}$, em que $\left|S_{0}\right|$ representa o total de documentos do subconjunto $S_{0}$.

- Na análise experimental foram gerados 22.750 dendrogramas para cada coleção textual, incluindo 250 dendrogramas de referência (informação privilegiada) e 22.500 dendrogramas provenientes da informação técnica. Ao longo da avaliação experimental foram extraídos um total de 227.500 dendrogramas.

O primeiro aspecto analisado na avaliação experimental é a eficácia de cada índice na tarefa seleção de modelos de agrupamentos. Esses resultados são descritos por meio de uma matriz VED (Vitória, Empate e Derrota), conforme ilustrado na Tabela 5.1. A matriz VED compara o algoritmo da linha contra o algoritmo da coluna. Por exemplo, considere a segunda linha e primeira coluna da matriz VED para a coleção textual 20ng. Os valores [60.0 0.0 40.0] desta célula indicam, respectivamente, que a abordagem cdi-MAX - quando comparada com a aRi-MAX - selecionou um melhor modelo de agrupamento em $60 \%$ das execuções, não houve empates e que selecionou um pior modelo de agrupamento em $40 \%$ das execuções. Nesse caso, empate significaria que as duas abordagens selecionaram o mesmo modelo de agrupamento.

A partir das matrizes VED é possivel verificar que a abordagem cdi-MAX foi mais promissora na seleção de modelos, obtendo-se a maior porcentagem de 
vitórias em cinco coleções: 20ng, Hitech, LATimes, PubMed-Cancer e Reviews; enquanto foi derrotada pela aRi-MAX ou edi-MAX em três coleções: $N S F$, Re8 e WebACE. Nas coleções $A C M$ e Ohsumed400 há maior predomínio de empate entre as abordagens.

É importante observar que nos três casos em que a cdi-MAX obteve maior porcentagem de derrotas ( NSF, Re8 e WebACE), a abordagem edi-MAX obteve maior porcentagem de vitórias do que a $a$ Ri-MAX. Esse aspecto fornece indícios de que as abordagens que utilizam informação da estrutura hierárquica tendem a ter um melhor desempenho na seleção de modelos. De fato, quando é considerado apenas as vitórias e derrotas, a abordagem $a R i-M A X$ não obteve melhor desempenho em nenhuma das coleções textuais.

As matrizes VED são úteis para comparar a quantidade de vezes que uma abordagem ganha/empata/perde de outra na tarefa de seleção de modelos, mas também é necessário analisar a qualidade dos modelos de agrupamento selecionados. Nesse sentido, na Figura 5.4 é ilustrada uma comparação da acurácia (índice $F_{S C O R E}$ ) dos modelos de agrupamento obtida por cada abordagem, desde o modelo inicial até o modelo final obtido após o agrupamento incremental.

Nos gráficos da Figura 5.4 é possivel notar que a escolha do modelo inicial é importante para todo o processo de agrupamento. Na maioria dos casos, a abordagem que seleciona o melhor modelo inicial $\left(S_{0}\right)$ também obtém o melhor acurácia na solução final de agrupamento $\left(S_{10}\right)$. Isto ocorreu com a abordagem cdi-MAX que apresentou maiores médias para valores $F_{S C O R E}$, exceto nas coleções $N S F$, Re8 e WebACE. Considerando o índice $F_{S C O R E}$, as abordagens aRi-MAX e edi-MAX obtiveram desempenho similar nas coleções textuais analisadas.

Um outro aspecto importante a ser analisado é sobre o quanto o uso da informação privilegiada para seleção de modelos melhora o processo de agrupamento, quando comparado a uma estratégia Baseline, ou seja, o processo de agrupamento hierárquico tradicional baseado apenas na informação técnica. Na Figura 5.5 é apresentado um gráfico radar com a porcentagem de melhora (ou piora) obtido por cada abordagem em relação ao Baseline.

Em algumas coleções textuais, o uso da informação privilegiada para seleção de modelos apresenta uma solução final de agrupamento com média $F_{S C O R E}$ levemente superior (e algumas vezes inferior) do que o Baseline, em especial, nas abordagens aRi-MAX e edi-MAX. Nesses casos, embora a informação privilegiada represente uma informação adicional e valiosa a respeito 
((a)) 20ng

\begin{tabular}{l|ccc|ccc|ccc}
\hline \hline & \multicolumn{3}{|c|}{ aRi-MAXX } & \multicolumn{3}{c|}{ cdi-MAX } & \multicolumn{3}{c}{ edi-MAX } \\
& $\mathbf{v}$ & $\mathbf{e}$ & $\mathbf{d}$ & $\mathbf{v}$ & $\mathbf{e}$ & $\mathbf{d}$ & $\mathbf{v}$ & $\mathbf{e}$ & $\mathbf{d}$ \\
\hline \hline aRi-MAX & & - & & 40.0 & 0.0 & 60.0 & 60.0 & 10.0 & 30.0 \\
cdi-MAX & 60.0 & 0.0 & 40.0 & & - & & 40.0 & 60.0 & 0.0 \\
edi-MAX & 30.0 & 10.0 & 60.0 & 0.0 & 60.0 & 40.0 & & - & \\
\hline
\end{tabular}

((b)) ACM

\begin{tabular}{l|ccc|ccc|ccc}
\hline aRi-MAX & & - & & 29.6 & 35.7 & 34.8 & 23.5 & 47.5 & 29.0 \\
cdi-MAX & 34.8 & 35.7 & 29.6 & & - & & 31.6 & 38.0 & 30.4 \\
edi-MAX & 29.0 & 47.5 & 23.5 & 30.4 & 38.0 & 31.6 & & - & \\
\hline
\end{tabular}

((c)) Hitech

\begin{tabular}{l|ccc|ccc|ccc}
\hline aRi-MAX & & - & & 27.7 & 34.7 & 37.6 & 19.0 & 55.1 & 25.9 \\
cdi-MAX & 37.6 & 34.7 & 27.7 & & - & & 34.8 & 32.4 & 32.8 \\
edi-MAX & 25.9 & 55.1 & 19.0 & 32.8 & 32.4 & 34.8 & & - & \\
\hline
\end{tabular}

((d)) LATimes

\begin{tabular}{|c|c|c|c|c|c|c|c|c|c|}
\hline $\begin{array}{l}\text { aRi-MAX } \\
\text { cdi-MAX } \\
\text { edi-MAX }\end{array}$ & $\begin{array}{l}48.2 \\
18.6\end{array}$ & $\begin{array}{l}\overline{23.3} \\
63.8\end{array}$ & $\begin{array}{l}28.6 \\
17.6\end{array}$ & 28.6 & $\begin{array}{c}23.3 \\
\overline{19.3}\end{array}$ & 48.2 & $\begin{array}{l}17.6 \\
48.5\end{array}$ & $\begin{array}{c}63.8 \\
19.3 \\
-\end{array}$ & $\begin{array}{l}18.6 \\
32.2\end{array}$ \\
\hline \multicolumn{10}{|c|}{ ((e)) NSF } \\
\hline $\begin{array}{l}\text { aRi-MAX } \\
\text { cdi-MAX } \\
\text { edi-MAX }\end{array}$ & $\begin{array}{l}36.4 \\
47.3\end{array}$ & $\begin{array}{l}5.5 \\
7.3\end{array}$ & $\begin{array}{l}58.2 \\
45.5\end{array}$ & 58.2 & $\begin{array}{c}5.5 \\
\overline{34.5}\end{array}$ & $\begin{array}{l}36.4 \\
30.9\end{array}$ & $\begin{array}{l}45.5 \\
30.9\end{array}$ & $\begin{array}{c}7.3 \\
34.5 \\
-\end{array}$ & $\begin{array}{l}47.3 \\
34.5\end{array}$ \\
\hline
\end{tabular}

((f)) Ohsumed400

\begin{tabular}{c|ccc|ccc|ccc}
\hline aRi-MAX & & $-\overline{-}$ & & 25.0 & 46.2 & 28.8 & 19.2 & 61.5 & 19.2 \\
cdi-MAX & 28.8 & 46.2 & 25.0 & & - & & 28.8 & 48.1 & 23.1 \\
edi-MAX & 19.2 & 61.5 & 19.2 & 23.1 & 48.1 & 28.8 & & - & \\
\hline
\end{tabular}

((g)) PubMed-Cancer

\begin{tabular}{c|ccc|ccc|ccc}
\hline aRi-MAX & & - & & 27.8 & 33.3 & 38.9 & 22.2 & 44.4 & 33.3 \\
cdi-MAX & 38.9 & 33.3 & 27.8 & & - & & 27.8 & 50.0 & 22.2 \\
edi-MAX & 33.3 & 44.4 & 22.2 & 22.2 & 50.0 & 27.8 & & - & \\
\hline
\end{tabular}

((h)) $\operatorname{Re} 8$

\begin{tabular}{c|ccc|ccc|ccc}
\hline aRi-MAX & & - & & 66.9 & 11.7 & 21.4 & 25.3 & 40.9 & 33.8 \\
cdi-MAX & 21.4 & 11.7 & 66.9 & & - & & 18.8 & 14.3 & 66.9 \\
edi-MAX & 33.8 & 40.9 & 25.3 & 66.9 & 14.3 & 18.8 & & - & \\
\hline
\end{tabular}

((i)) Reviews

\begin{tabular}{c|ccc|ccc|ccc}
\hline aRi-MAX & & $\overline{-}$ & & 22.5 & 37.2 & 40.3 & 49.6 & 33.1 & 17.3 \\
cdi-MAX & 40.3 & 37.2 & 22.5 & & - & & 65.3 & 20.6 & 14.1 \\
edi-MAX & 17.3 & 33.1 & 49.6 & 14.1 & 20.6 & 65.3 & \multicolumn{4}{|c}{-} & \\
\hline
\end{tabular}

((j)) WebACE

\begin{tabular}{c|ccc|ccc|ccc}
\hline aRi-MAX & & - & & 39.5 & 21.2 & 39.3 & 22.2 & 54.9 & 22.9 \\
cdi-MAX & 39.3 & 21.2 & 39.5 & & - & & 41.2 & 16.7 & 42.1 \\
edi-MAX & 22.9 & 54.9 & 22.2 & 42.1 & 16.7 & 41.2 & & - & \\
\hline
\end{tabular}

Tabela 5.1: Tabela com matrizes VED (Vitória, Empate e Derrota) entre as abordagens em cada coleção textual. Em cada matriz VED, compara-se a abordagem da linha contra a abordagem da coluna. Os valores $v, e, d$, representam, respectivamente, as porcentagens de vitória, empate e derrota. 


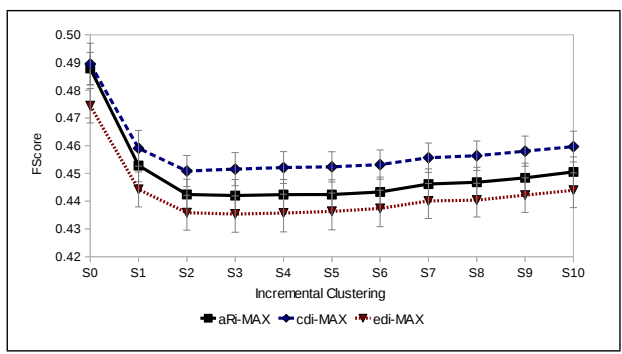

((a)) 20ng

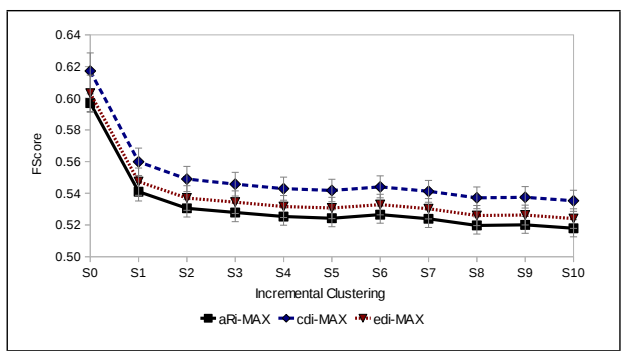

((c)) Hitech

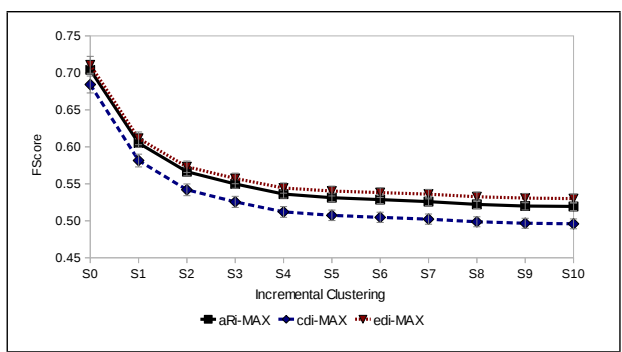

((e)) NSF

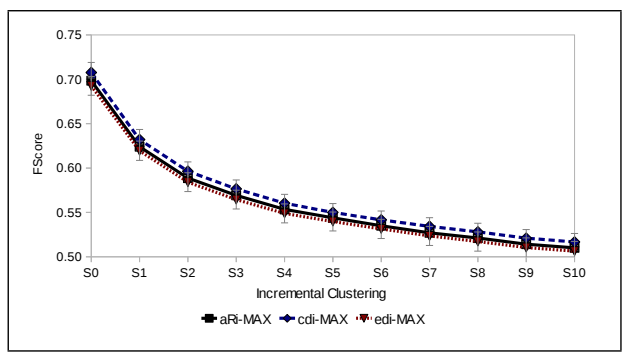

((g)) PubMed-Cancer

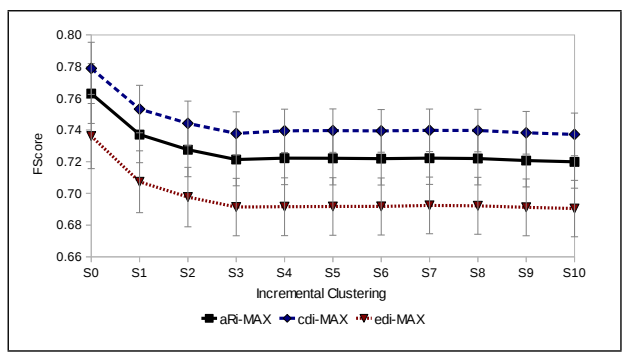

((i)) Reviews

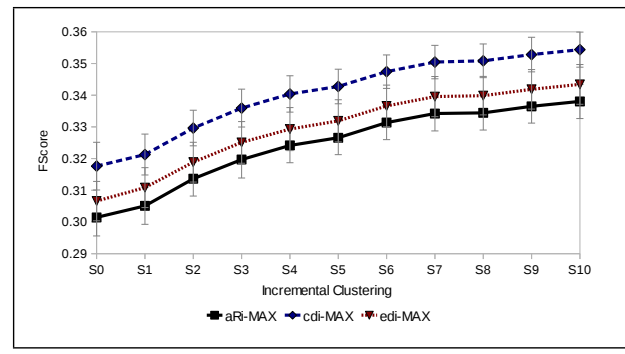

((b)) ACM

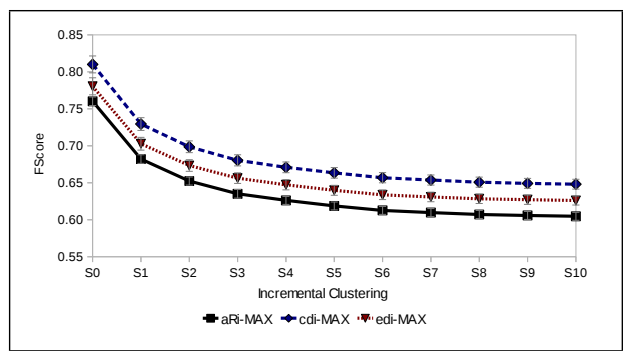

((d)) LATimes

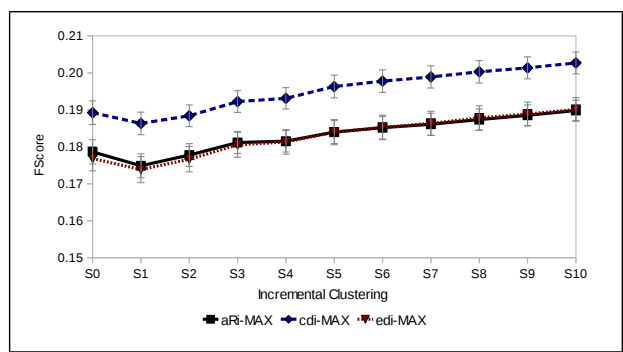

((f)) Ohsumed400

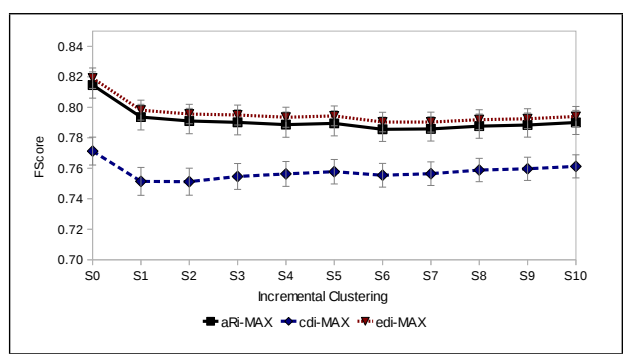

((h)) $\operatorname{Re} 8$

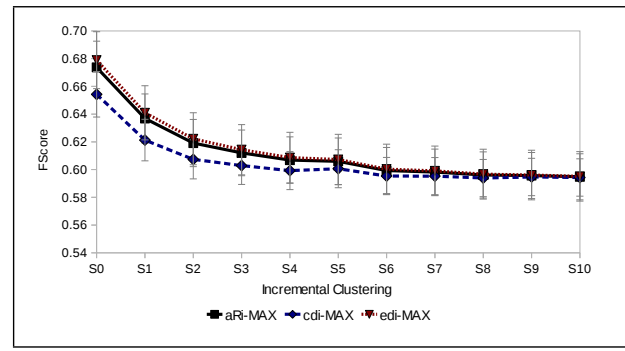

((j)) WebACE

Figura 5.4: Comparação do índice $F_{S C O R E}$ das abordagens aRi-MAX, cdi-MAX e edi-MAX durante o processo de agrupamento. A etapa $S_{0}$ representa a acurácia do modelo inicial e $S_{1} 0$ a acurácia da solução final de agrupamento. 
dos dados, seu uso em abordagens de seleção de modelos é limitado. Esta informação adicional não é incorporada de fato nos dados, mas é empregada de forma indireta como uma estrutura de referência. Nas abordagens baseadas em aprendizado de métricas (IPIML), por exemplo, a informação privilegiada permite alterar/refinar a estrutura de agrupamento por meio de nova(s) métrica(s). Já com a seleção de modelos, mesmo que o conjunto de modelos de agrupamento sejam todos "ruins", um deles sempre será selecionado.

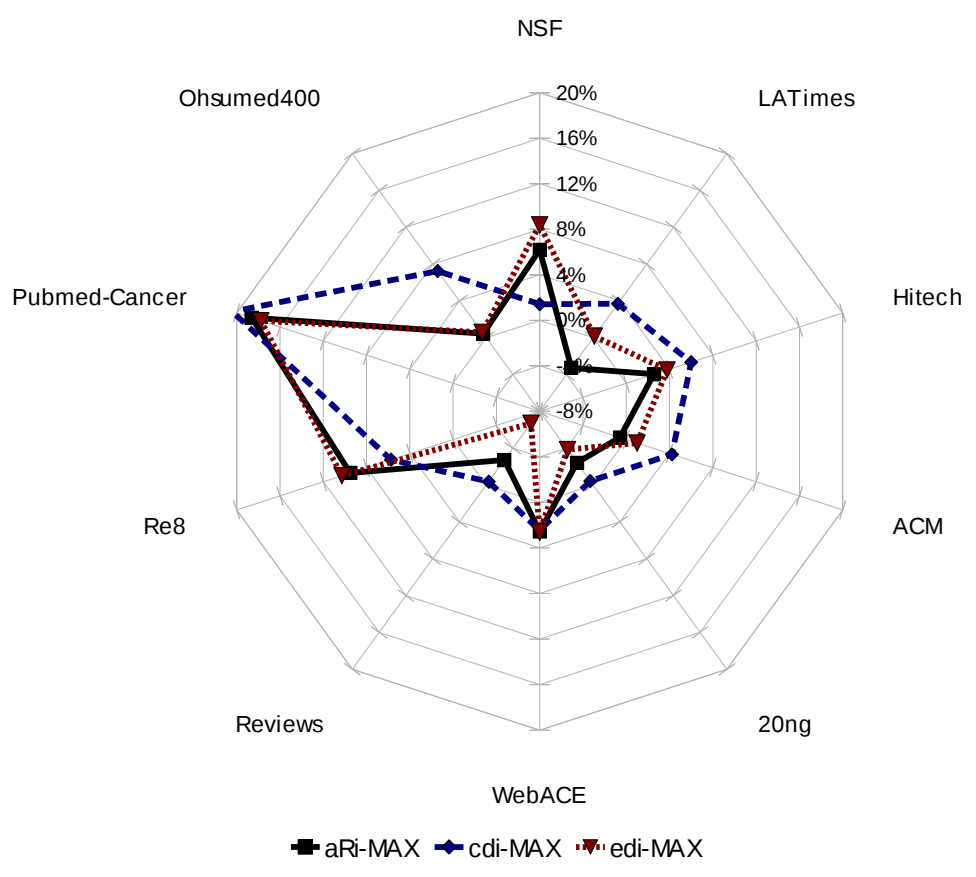

Figura 5.5: Porcentagem de ganho/perda das abordagens aRi-MAX, cdi-MAX e edi-MAX em relação ao Baseline.

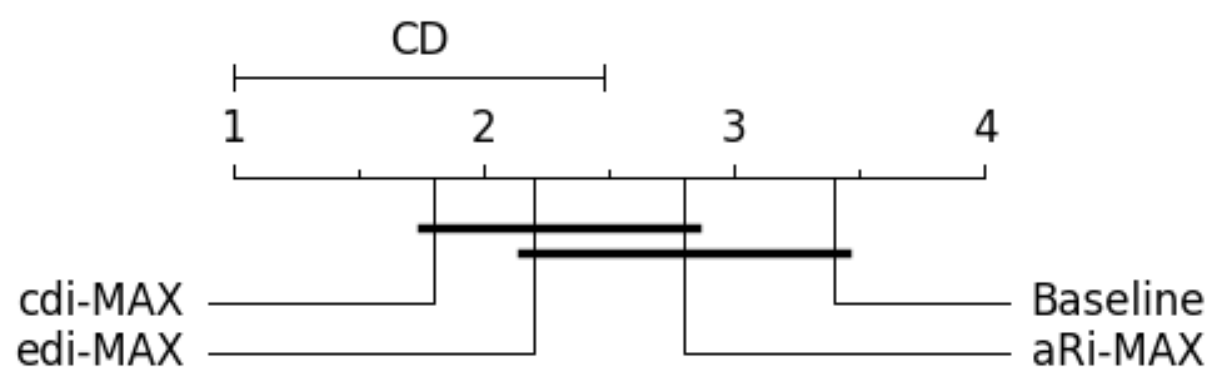

Figura 5.6: Diferença crítica $(C D)$ sobre o ranking das abordagens exploradas de acordo com a acurácia $\left(F_{S C O R E}\right)$ da solução final de agrupamento.

Para verificar se o uso de informação privilegiada para seleção de modelos é significativamente superior ao Baseline, foi realizada uma análise estatística 
com o teste não paramétrico de Friedman e pós-teste de Nemenyi (com 95\% de confiança), conforme recomendações de Demšar (2006) e Japkowicz e Shah (2011). Uma representação gráfica dos resultados da análise estatística está ilustrada na Figura 5.6, em que as abordagens estão ordenadas conforme sua posição no raking. Este ranking é calculado considerando os valores médios do índice $F_{S C O R E}$, dentre as diversas execuções realizadas. Uma abordagem é considerada estatisticamente superior (ou inferior) a outra se a diferença crítica CD (Critical Difference) entre elas é maior do que a CD de referência computada pelo teste.

A análise estatística revela dois grupos sem diferença significativa em seus desempenhos: [cdi-MAX, edi-MAX, aRi-MAX] e [edi-MAX, aRi-MAX, Baseline]. Há uma diferença estatística significativa apenas entre as abordagens cdi-MAX e Baseline, fornecendo evidências de que o descritor $C D$ é mais indicado para apoiar a seleção de modelos com uso de informação privilegiada. Esta informação também indica que não apenas a topologia da estrutura hierárquica extraída da informação privilegiada é importante, mas também os valores de distância entre seus exemplos devem ser considerados durante a seleção de modelos.

\subsection{Considerações Finais}

Neste capítulo foi introduzido o uso de informação privilegiada para seleção de modelos de agrupamento hierárquico. Além da adaptação e avaliação de uma abordagem recentemente proposta na literatura, denominada aRi-MAX, também foram propostos duas novas abordagens, cdi-MAX e edi-MAX, que possuem o diferencial de extrair descritores da estrutura hierárquica durante a seleção de modelos.

Os resultados experimentais fornecem evidências de que a abordagem cdiMAX é mais adequada para seleção de modelos com apoio de informação privilegiada. No cenário avaliado foi utilizado o algoritmo de agrupamento hierárquico Bisecting $k$-means, com similaridade baseada no cosseno, para geração de todos os dendrogramas. O uso de uma mesma estratégia de agrupamento e uma mesma medida de similaridade permite a comparação dos respectivos dendrogramas de forma mais direta com uso da correlação de Pearson, que é utilizado para cálculo do índice cdi (Cophenetic Difference Index). Em cenários envolvendo diferentes medidas de similaridade, bem como diferentes algoritmos de agrupamento, a abordagem edi-MAX mé mais recomendada, embora 
este cenário não tenha sido explorado nos experimentos. Os valores de matrizes de descritores provenientes de diferentes medidas e algoritmos potencialmente terão magnitudes e distribuições diferentes, cenário que favorece o uso critérios baseados na correlação de Spearman para comparação dessas matrizes.

Uma limitação no uso de informação privilegiada para seleção de modelos é que tal informação adicional não é incorporada, de fato, no modelo inicial, ou seja, não há uma estratégia para refinar/corrigir o modelo selecionado. Por outro lado, possui a vantagem de que uma gama de algoritmos de agrupamento disponiveis na literatura possam ser facilmente utilizados, sem a necessidade de desenvolver um algoritmo específico. 



\section{CAPÍTULO}

\section{6}

\section{Aplicações}

\subsection{Considerações Iniciais}

Nos Capítulos 3, 4 e 5 foram propostas e avaliadas diferentes abordagens para incorporação de informação privilegiada em agrupamento hierárquico de textos. A análise das abordagens foi realizada com foco nos algoritmos e aspectos teóricos envolvidos, com avaliação experimental baseada em coleções textuais de benchmark. Neste capítulo são discutidos alguns aspectos práticos em relação ao uso de informação privilegiada para agrupamento de textos. Em especial, são apresentadas duas aplicações: (i) análise exploratória de mídias sociais e (ii) sistemas de recomendação sensiveis ao contexto.

As aplicações foram desenvolvidas em colaboração com pesquisadores de outras áreas, que identificaram nas abordagens investigadas neste projeto uma oportunidade de avançar em suas respectivas linhas de pesquisa. Embora cada aplicação tenha sido avaliada de acordo com o contexto original em que foram propostas (em suas respectivas linhas de pesquisa), neste capítulo buscou-se enfatizar sobre como modelos de agrupamento hierárquico usando informação privilegiada foram empregados nessas aplicações.

\subsection{Análise Exploratória de Mídias Sociais}

A popularização das mídias sociais, a exemplo de fóruns, blogs e redes sociais, tem fornecido novas formas de interação social, como a criação e compartilhamento de informação em vários formatos. Uma quantidade significativa dessa informação é armazenada no formato textual. Recentemente, 
o conteúdo textual de acesso público disponibilizado nessas mídias sociais tem sido utilizado por pesquisadores da área de Ciência Sociais para estudar diversos temas relacionados à sociedade. Nesse sentido, surgiu uma direção de pesquisa denominada "Ciência Social Computacional", discutida em Lazer et al. (2009). A ideia geral é de que esta grande quantidade de dados pode ser utilizada para analisar o comportamento da sociedade de forma não intrusiva (Kozinets, 2010).

Uma técnica empregada pelos Cientistas Sociais é conhecida como Discurso do Sujeito Coletivo (DSV) (Lefevre e Lefevre, 2006), que tem o objetivo de encontrar discursos que representam uma ideia coletiva por meio da análise de depoimentos individuais. Tais discursos são gerados agregando-se trechos de depoimentos individuais que apresentam o mesmo argumento. Assim, com a técnica de Discurso do Sujeito Coletivo, espera-se verificar qual o pensamento de uma comunidade a respeito de um assunto, bem como o grau de compartilhamento das opiniões da comunidade sobre este assunto. Para que a técnica obtenha resultados satisfatórios, é necessário um considerável esforço dos analistas, uma vez que dentre uma grande quantidade de textos apenas uma parcela é de interesse. Selecionar esta parcela de textos é, então, um dos desafios existentes para a análise (Carvalho et al., 2012a).

A aplicação apresentada nesta seção foi desenvolvida para apoiar um grupo de pesquisadores ${ }^{1}$ da PUC-Rio (Pontifícia Universidade Católica do Rio de Janeiro) que, por sua vez, atuavam junto ao Instituto de Responsabilidade Social Sírio-Libanês. Este grupo busca aplicar a técnica DSV para identificar motivações de pessoas sobre o início e o fim do consumo de drogas. Para tal, o estudo analisa discussões em comunidades virtuais na Internet sobre o uso de crack, cocaína e maconha (Instituto-Sírio-Libanês, 2012).

Em um primeiro momento, a DSV foi empregada pelos analistas de forma manual. Foi utilizada uma coleção textual extraída de comunidades virtuais da rede social Orkut ${ }^{\circledR}$ composta por 8655 mensagens coletadas em Setembro de 2011. As mensagens são provenientes de 434 participantes (57\% homens e $43 \%$ mulheres) e distribuídas em 384 tópicos de discussão. Após o emprego da técnica de Discurso do Sujeito Coletivo, os analistas selecionaram um conjunto de opiniões sumarizadas em 39 tópicos e 925 mensagens (Instituto-SírioLibanês, 2012).

Devido ao grande esforço humano envolvido no processo descrito acima, uma ferramenta para apoiar a análise exploratória de mídias sociais, deno-

\footnotetext{
${ }^{1}$ O grupo foi coordenado pelo Prof. Carlos José Pereira de Lucena durante o trabalho de doutorado do Prof. Dárlinton Barbosa Feres Carvalho
} 
minada TorchSR (Carvalho et al., 2012b, 2014), foi desenvolvida em conjunto com o grupo de pesquisadores da PUC-RIO para atender a demanda da área. A ferramenta é um módulo adaptado do ambiente Torch ${ }^{2}$, introduzido em Marcacini e Rezende (2010), que atualmente contém diversos algoritmos de préprocessamento e agrupamento de textos (incluindo as abordagens propostas neste projeto de doutorado). Os passos básicos da aplicação são descritos a seguir.

\subsubsection{Extração da Informação Privilegiada}

Uma representação textual complementar à bag-of-words (informação técnica) foi obtida com base em itemsets. No caso específico desta aplicação, foram obtidos apenas itemsets de tamanho 2, ou seja, termos compostos por duas palavras. Isto foi necessário porque a interface de interação com os usuários para efetuar a seleção de termos é baseada em uma rede de coocorrência de termos. Nesta rede, cada palavra é vértice de um grafo e as arestas representam os itemsets. O grafo é direcionado, em que o vértice de origem é a palavra com maior valor de frequência. Assim, a rede de coocorrência de termos permite identificar a relação de subsunção entre os termos (Lawrie et al., 2001). Para exemplificar, na Figura 6.1 é apresentada parte da rede de coocorrência da coleção textual utilizada nesta aplicação. Na parte esquerda, são listados os itemsets extraídos para seleção pelos usuários. Na parte central, é apresentada a rede de coocorrência, que é atualizada conforme os itemsets são selecionados. Na parte da direita, são apresentadas configurações para facilitar a visualização da rede de coocorrência de termos.

Ao finalizar esta etapa, a representação textual de informação privilegiada é obtida com base no conjunto de itemsets selecionado pelo usuário. Por fim, é obtido o modelo de agrupamento hierárquico combinando-se informação técnica e informação privilegiada, por exemplo, com uso da abordagem LIHC.

\subsubsection{Seleção de Conteúdo para Discurso do Sujeito Coletivo}

A identificação de conteúdo que seja relevante para o Discurso do Sujeito Coletivo (DSV) é o objetivo principal da análise exploratória. No entanto, como não há um conhecimento prévio do que se deseja buscar nos textos, é difícil para os usuários definirem critérios de busca. Nesta aplicação, o modelo de

\footnotetext{
${ }^{2}$ Torch: http://sites.labic.icmc.usp.br/
} 


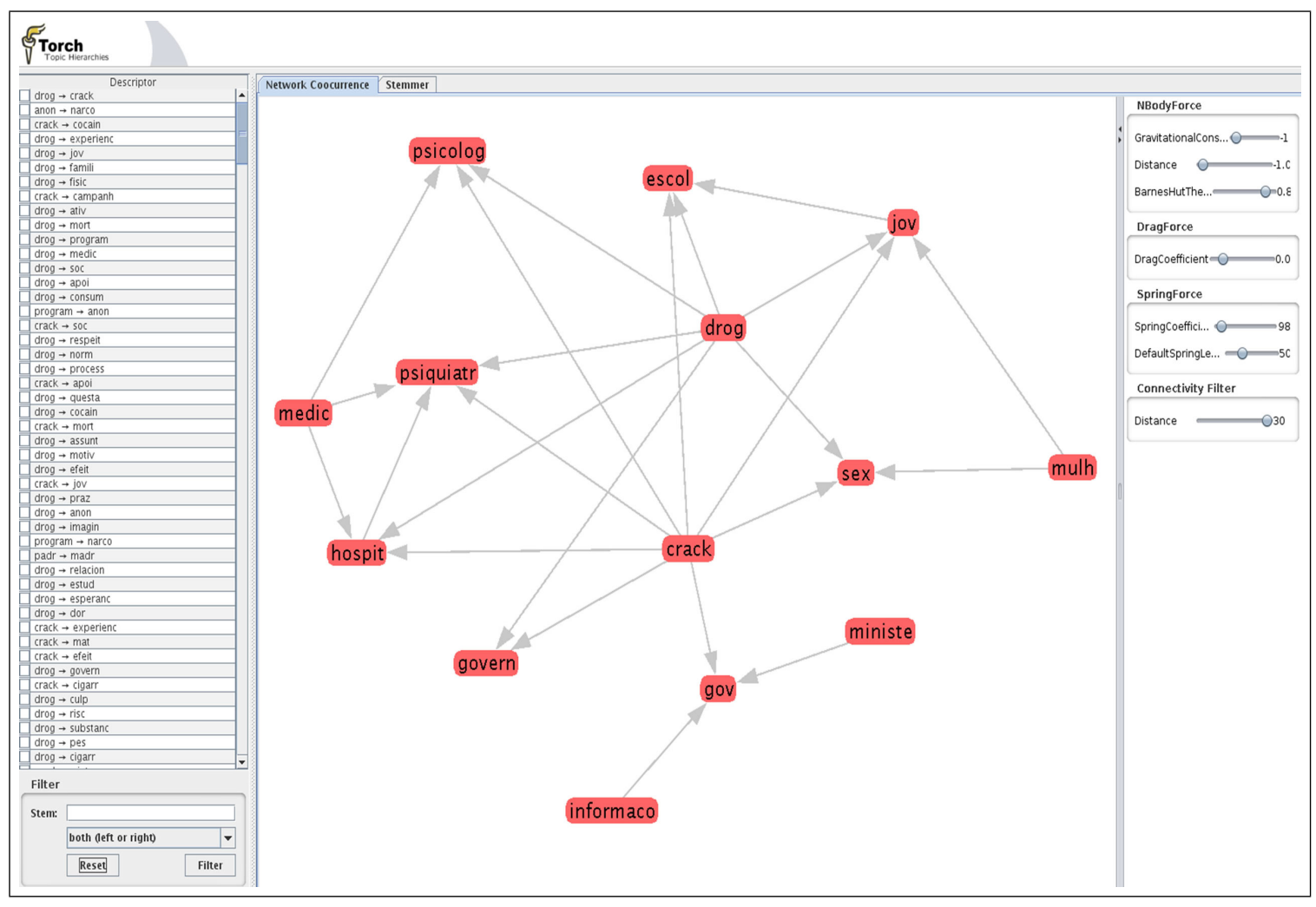

Figura 6.1: Interface para seleção de itemsets e exploração da rede de coocorrência de termos da ferramenta TorchSR.

agrupamento hierárquico foi utilizado para guiar a análise exploratória, em que cada grupo e subgrupo possui um descritor que indica o significado das mensagem ali agrupadas. Esta organização está relacionada com a hipótese de que se o usuário está interessado em um documento de um determinado grupo, também deve estar interessado em outros desse grupo ou de seus subgrupos (Manning et al., 2008).

Na Figura 6.2 é ilustrada a interface de seleção de contéudo utilizada nesta aplicação. O modelo de agrupamento hierárquico e os respectivos descritores são apresentado na parte A da Figura 6.2. Após selecionar um dos (sub)grupos, a ferramenta apresenta os tópicos das comunidades virtuais na parte $\mathrm{B}$, ordenados de acordo com sua similaridade ao grupo selecionado. $\mathrm{O}$ usuário, então, pode selecionar alguns tópicos de discussão que são de interesse para a análise. Para cada tópico selecionado, são listadas as mensagens mais similares ao assunto geral do tópico. Esta operação é realizada na parte $\mathrm{C}$, onde as mensagens selecionadas também são consideradas para a técnica DSV. Já na parte D da Figura 6.2, é apresentada uma visão geral dos textos que foram selecionados até aquele momento. Por fim, é importante lembrar 


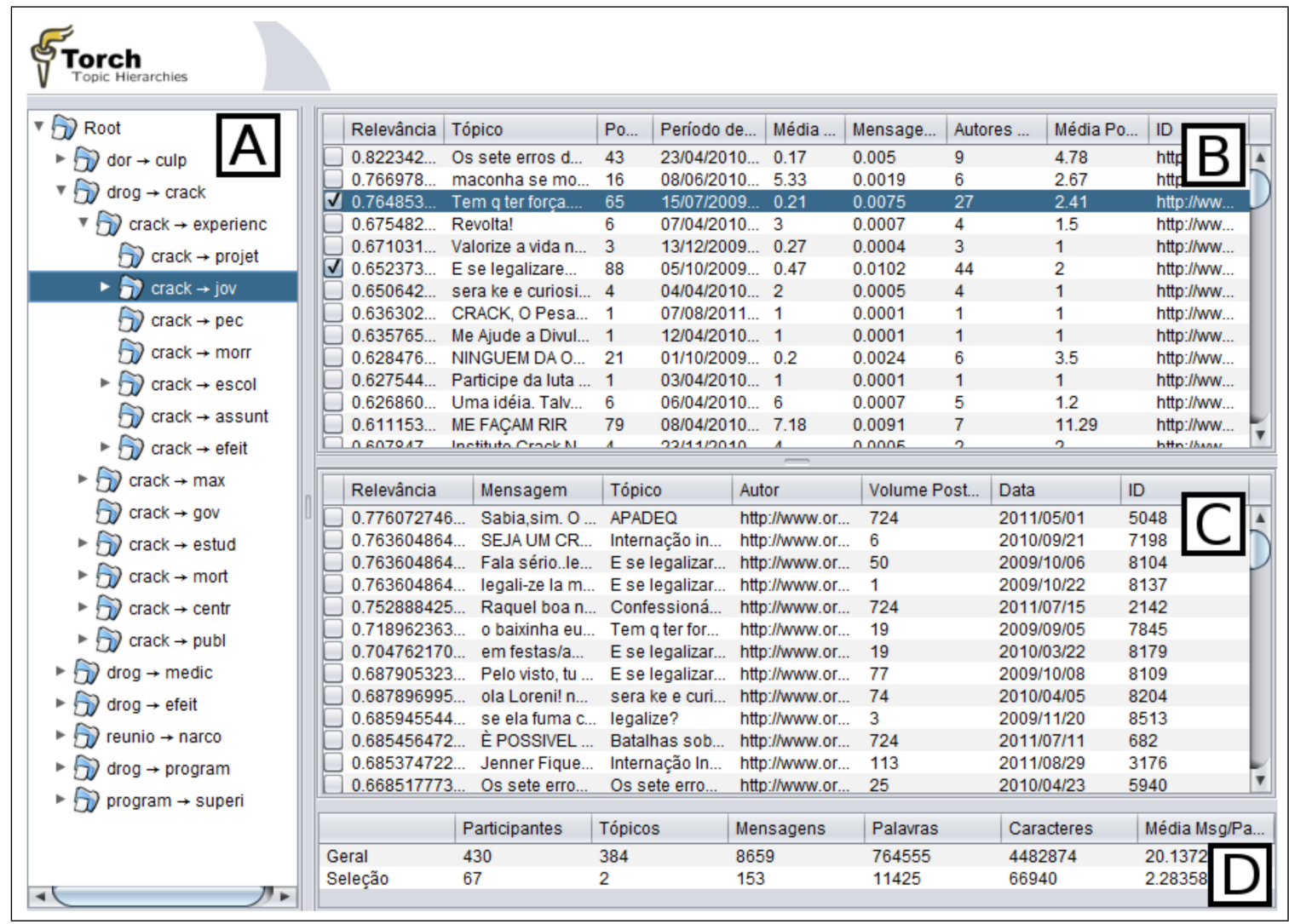

Figura 6.2: Interface para apoiar a seleção de conteúdo textual a ser utilizado para análise do Discurso do Sujeito Coletivo (DSV)

que o usuário pode voltar à etapa anterior, incluir novos termos na representação de informação privilegiada, obter um novo agrupamento hierárquico e continuar o processo de seleção de conteúdo.

Um detalhe importante em relação ao uso do agrupamento hierárquico para apoiar a seleção de conteúdo é sobre a estrutura do dendrograma. Por ser uma árvore binária, o dendrograma possui muitos grupos e subgrupos que dificultam a análise exploratória para os usuários. Nesse sentido, foi utilizado um método para refinamento de dendrogramas (Marcacini et al., 2012a). O método é denominado "fusão" e essencialmente calcula a proximidade entre grupos pais e filhos por meio da similaridade cosseno entre seus respectivos centroides. Se esta similaridade for muito alta (maior do que um limiar definido pelo usuário), então o grupo filho não "especializa" o conhecimento existente no grupo pai. Nesses casos, o grupo filho é removido da hierarquia e os grupos netos são promovidos. Esse processo é iterativo e continua até que todas as similaridades sejam menores do que o limiar definido pelo usuário. Os resultados experimentais apresentados em Marcacini et al. (2012a) indicam que o método proposto fusão permite reduzir a estrutura do agru- 
pamento hierárquico, sem afetar significativamente a acurácia (indice $F_{S C O R E}$ ) do modelo.

\subsubsection{Discussão dos Resultados}

A ferramenta desenvolvida para análise exploratória de mídias sociais foi avaliada qualitativamente. Para tal, o grupo de pesquisadores da PUC-Rio, por meio do Laboratório de Engenharia de Software (LES), realizou uma oficina $^{3}$ de "Pesquisa Qualitativa" de quatro horas de duração, onde os participantes receberam um treinamento em Pesquisa Qualitativa com ênfase em análise de conteúdo e discurso. Uma das atividades da oficina foi a avaliação da ferramenta TorchSR. O objetivo da avaliação foi verificar o desempenho de modelos de agrupamento hierárquico para apoiar seleção de conteúdo relevante quando comparado com uma estratégia manual. Embora os resultados da avaliação demonstraram que a TorchSR auxilia nesta tarefa, a estratégia de avaliação não permite analisar o efeito da informação privilegiada neste processo.

Um dos aspectos interessantes nessa aplicação é o uso da informação privilegiada não apenas para melhorar o aprendizado de modelos de agrupamento, mas também para facilitar a visualização do modelo. A informação privilegiada, nesse caso, permite facilitar a interpretação dos grupos e subgrupos quando seus termos são utilizados como descritores.

Por fim, também é importante ressaltar que foi necessário adaptar a interface de seleção interativa de termos de acordo com a aplicação. Em alguns cenários, é possivel utilizar fontes externas para enriquecer ainda mais esse processo, como consulta a dicionários de domínio, ontologias, entidades nomeadas e Wikipedia.

Os resultados obtidos com esta aplicação foram utilizados como parte da tese de doutorado de Carvalho (2013).

\subsection{Sistemas de Recomendação Sensiveis ao Contexto}

Com a grande variedade de produtos e serviços disponíveis na web, sistemas de recomendação têm sido propostos para identificar itens que sejam

\footnotetext{
${ }^{3}$ A Oficina de Pesquisa Qualitativa foi realizada nas dependência da PUC-Rio no dia 12 de Novembro de 2013.
} 
de maior interesse para os usuários (Ricci et al., 2011). Nesse sentido, a recomendação de itens se tornou popular principalmente em sites de comércio eletrônico, interagindo diretamente com os usuários (consumidores) por meio de sugestões dos produtos e serviços. Empresas como Amazon ${ }^{4}$, Netflix ${ }^{5}$ e Last.fm ${ }^{6}$ são exemplos de sucesso onde o uso de sistemas de recomendação permitiu aumento significativo de suas vendas (Ricci et al., 2011).

A maioria dos sistemas de recomendação é baseada no histórico de acesso dos usuários aos itens para realizar recomendações (Ekstrand et al., 2011). No entanto, em muitas aplicações, também é importante incorporar informação contextual no processo de recomendação (Adomavicius et al., 2005; Li et al., 2010; Bobadilla et al., 2013). Vários estudos têm demonstrado que a acurácia da recomendação aumenta de forma significativa quando informações (adicionais) de contexto, como horário e região, também são utilizadas durante a construção do modelo de recomendação (Adomavicius e Tuzhilin, 2011).

A "informação contextual" é um conceito que pode assumir diferentes definições dependendo do tipo de aplicação. No entanto, a definição mais usada é sugerida por Dey (2001): “Contexto é alguma informação que pode ser utilizada para caracterizar a situação de uma entidade. Uma entidade é uma pessoa, lugar ou objeto". Sistemas de recomendação sensíveis ao contexto permitem incorporar esta informação contextual no processo de recomendação. Embora estes sistemas tenham recebido grande atenção nos últimos anos, ainda existe um desafio recente em relação a métodos automáticos para extrair e estruturar a informação contextual.

As abordagens propostas nesta tese de doutorado, especialmente a abordagem LIHC (LUPI-Based Incremental Hierarchical Clustering), foram utilizadas para apoiar sistemas de recomendação sensíveis ao contexto. O objetivo principal é extrair informação contextual a partir do modelo de agrupamento hierárquico. O conjunto de itens do sistema de recomendação é organizado em grupos e subgrupos, no qual itens similares (com base em dados textuais) são alocados em um mesmo grupo. Assim, cada grupo e subgrupo é utilizado como uma possivel informação contextual para o sistema de recomendação. Nas próximas seções, este processo é discutido com mais detalhes.

\footnotetext{
${ }^{4}$ http://www.amazon.com

${ }^{5}$ http://www.netflix.com

${ }^{6}$ http://www.last.fm
} 


\subsubsection{Entidades Nomeadas como Informação Privilegiada}

A grande disponibilidade de dados textuais associados aos itens e usuários tem motivado o uso de técnicas de mineração de textos para extração da informação contextual. Uma das técnicas populares é o Reconhecimento de Entidades Nomeadas (REN), que consiste em identificar nomes próprios como pessoas, organizações, lugares e produtos, bem como dados temporais e expressões numéricas (Nadeau e Sekine, 2007). As técnicas para REN são computacionalmente custosas e necessitam de um conjuntos de regras ou base de conhecimento, ou seja, uma informação externa a respeito dos dados. Ainda, há técnicas para REN que são específicas para um domínio, baseadas em ontologias, dicionários de dados, e até mesmo com interação de usuários por meio de aprendizado ativo (Nadeau e Sekine, 2007; Ekbal et al., 2014).

Em vista disso, o uso de entidades nomeadas como um tipo de informação privilegiada foi investigado em colaboração com o trabalho de Sinoara et al. (2014). Os resultados experimentais indicam que é possivel melhorar a qualidade do modelo de agrupamento incorporando-se entidades nomeadas como informação privilegiada, porém apenas para coleções textuais formadas por textos longos, aliado com uma ferramenta eficaz para Reconhecimento de Entidades Nomeadas.

Entre as coleções textuais exploradas em Sinoara et al. (2014), a coleção BestSports obteve os resultados mais promissores. Esta coleção é composta por relatórios (em português) sobre diferentes eventos esportivos e atletas. A informação técnica (bag-of-words) foi extraída por meio da ferramenta JPretext $^{7}$, remoção de stopwords, radicalização das palavras e remoção de termos que ocorrem em menos do que dois documentos. A extração da informação privilegiada foi apoiada pela ferramenta REMBRANDT ${ }^{8}$, um Reconhecedor de Entidades Nomeadas para a Língua Portuguesa. O REMBRANDT realiza consulta à informações externas como Wikipédia ${ }^{9}$ e DBpedia $^{10}$, permitindo a identificação mais robusta de entidades nomeadas. Após a etapa de préprocessamento, a informação técnica ficou composta por 1211 termos e 881 documentos. Já a informação privilegida ficou composta por 660 termos (entidades nomeadas) e 878 documentos. Para ilustrar esse processo, na Tabela 6.1 são apresentados os 20 termos mais frequentes de cada uma das representações de informação técnica e informação privilegiada.

\footnotetext{
${ }^{7}$ JPretext: http://sites.labic.icmc.usp.br/torch/msd2011/jpretext/

${ }^{8}$ REMBRANDT: http://xldb.di.fc.ul.pt/Rembrandt/

${ }^{9}$ Wikipédia: http://pt.wikipedia.org/

${ }^{10}$ DBpedia: http://pt.dbpedia.org
} 
Tabela 6.1: Lista de 20 termos mais frequentes das representações de informação técnica e informação privilegiada.

\begin{tabular}{l|l||l|l}
\hline \multicolumn{2}{c||}{ Informação Técnica } & \multicolumn{2}{c}{ Informação Privilegiada } \\
\hline \hline 1. brasil & $11 . \mathrm{min}$ & 1. brasil & 11. X_minutos \\
\hline 2. jog & 12. prov & 2. atenas & 12. jogos_pan \\
\hline 3. fic & 13. tec & 3. jogos & 13. X_kg \\
\hline 4. olimp & 14. americ & 4. sydney & 14. cuba \\
\hline 5. pont & 15. disput & 5. honda & 15. voleibol \\
\hline 6. final & 16. feminin & 6. estados_unidos & 16. shumacher \\
\hline 7. set & 17. prim & 7. alemanha & 17. X_americanos \\
\hline 8. conquist & 18. seleca & 8. ferrari & 18. X_anos \\
\hline 9. derrot & 19. equip & 9. X_km & 19. gustavo_kuerten \\
\hline 10. aten & 20. quart & 10. espanha & 20. china \\
\hline
\end{tabular}

Uma vez analisadas as situações em que entidades nomeadas podem ser empregadas como informação privilegiada, foi realizado um estudo sobre como utilizar o modelo de agrupamento resultante em sistemas de recomendação sensiveis ao contexto. O algoritmo base utilizado para esta tarefa foi o IBCF (Item-Based Collaborative Filtering) (Deshpande e Karypis, 2004), uma estratégia tradicional de recomendação baseada em filtragem colaborativa.

\subsubsection{Recomendação baseada em Filtragem Colaborativa}

Os sistemas de recomendação sensíveis ao contexto explorados neste trabalho são baseados no algoritmo IBCF (Item-Based Collaborative Filtering). Neste algoritmo, seja $m$ o número de usuários $U=\left\{u_{1}, u_{2}, \ldots, u_{m}\right\}$ e $n$ o número de itens que podem ser recomendados $I=\left\{i_{1}, i_{2}, \ldots, i_{n}\right\}$. O modelo obtido pelo IBCF é a matriz de similaridades entre todos os pares de itens. A medida de similaridade baseada no cosseno é comumente empregada para este caso, conforme definido na Equação 6.1:

$$
\operatorname{sim}\left(i_{1}, i_{2}\right)=\cos \left(\overrightarrow{i_{1}}, \overrightarrow{i_{2}}\right)=\frac{\overrightarrow{i_{1}} \cdot \overrightarrow{i_{2}}}{\left\|\overrightarrow{i_{1}}\right\| *\left\|\overrightarrow{i_{2}}\right\|}
$$

onde $\overrightarrow{i_{1}}$ e $\overrightarrow{i_{2}}$ são vetores que identificam quais usuários acessaram os itens. Nesse caso, se um usuário acessou o item $i$, então $\vec{i}$ terá o valor 1 referente à posição deste usuário. Caso contrário, terá valor 0 .

Assim, dado um usuário ativo $u_{a}$ e seu conjunto de itens observáveis $O \subseteq$ $I$, a recomendação é realizada da seguinte maneira. Primeiro, é obtido um conjunto $R$ de itens candidatos à recomendação, selecionando-se todos os itens que não estão no conjunto de itens observáveis ( $i \notin O$ ). Então, para cada item candidato $r \in R$, o score da recomendação é calculado de acordo com a Equação 6.2: 


$$
\operatorname{score}\left(u_{a}, O, r\right)=\frac{\sum_{i \in K_{r} \cap O} \operatorname{sim}(r, i)}{\sum_{i \in K_{r}} \operatorname{sim}(r, i)}
$$

onde $K_{r}$ representa o conjunto dos $k$ itens mais similares ao item candidato $r$. Por fim, os $N$ itens candidatos com maiores valores de score são recomendados ao usuário $u_{a}$.

Panniello e Gorgoglione (2012) propuseram uma abordagem para considerar contextos no processo de recomendação do IBCF, chamada Filter PoF (Contextual Post-Filtering Approach). Neste caso, a informação de contexto é utilizada para filtrar (remover) recomendações da lista com os top-N itens recomendados. A ideia geral é computar a probabilidade dos usuários acessarem itens em um determinado contexto. Mais formalmente, a probabilidade $P_{c}(u, i)$, de um usuário $u$ acessar um item $i$ em um contexto $c$, é computada pela Equação 6.3:

$$
P_{c}\left(u_{a}, i\right)=\frac{N u m_{c}(u, i)}{N u m_{c}(u)}
$$

em que $\operatorname{Num}_{c}(u, i)$ é o número de usuários $u$ que acessaram o item $i$ no contexto $c$, e $N u m_{c}(u)$ é o número total de usuários que acessaram algum item no contexto $c$. Com base nessa probabilidade, a abordagem Filter PoF filtra as recomendações baseadas em um limiar $P *$ da probabilidade $P_{c}\left(u_{a}, r\right)$, conforme a Equação 6.4:

$$
\operatorname{score}_{c}\left(u_{a}, O, i\right)= \begin{cases}\operatorname{score}_{c}\left(u_{a}, O, i\right), & \text { se } P_{c}\left(u_{a}, i\right) \geq P * \\ 0, & \text { caso contrário }\end{cases}
$$

A abordagem proposta LIHC (LUPI-Based Incremental Hierarchical Clustering) foi, então, utilizada para obter o agrupamento hierárquico incoporandose informação privilegiada (entidades nomeadas). Cada grupo e subgrupo do modelo obtido pelo LIHC é um possível contexto para o Filter PoF. Do ponto de vista prático, isto significa que a informação contextual está mapeando itens que possuem descrições textuais similares, incluindo as entidades nomeadas extraídas desses itens. Dessa forma, o sistema de recomendação baseado em contexto, além de tentar recomendar itens correlacionados em relação ao histórico de acesso dos usuários, também considera a similaridade dos itens de acordo com seu grupo (contexto) no modelo de agrupamento. 


\subsubsection{Discussão dos Resultados}

A eficácia do sistema de recomendação sensível ao contexto, com apoio da abordagem LIHC, foi avaliada em uma base de dados sobre agronegócios. A base possui 4.659 usuários, 15.037 acessos e 1.543 itens (boletins textuais em Português).

A informação técnica (bag-of-words) foi extraída por meio da ferramenta JPretext ${ }^{11}$, com remoção de stopwords, radicalização das palavras e remoção de termos que ocorrem em menos do que dois documentos. A extração da informação privilegiada foi apoiada pela ferramenta REMBRANDT ${ }^{12}$, um Reconhecedor de Entidades Nomeadas para a Língua Portuguesa.

Para análise dos resultados, foi considerado o algoritmo IBCF (que não considera informação de contexto) e a abordagem Filter PoF. Para a abordagem Filter PoF, a informação de contexto foi extraída de duas maneiras. A primeira utiliza apenas informação técnica, ou seja, a abordagem LIHC com Fator de Combinação $\alpha=0$. A segunda obtém modelos de agrupamento combinando informação técnica e informação privilegiada, com Fator de Combinação $\alpha=0.5$. Foi realizado um processo de ajuste de parâmetros, em que foram definidos empiricamente: $N=4$ para número de itens candidatos e $P *=0.1$ como valor de limiar da abordagem Filter PoF. Por fim, para definir o contexto, foram analisados grupos e subgrupos de diferentes granularidades. Uma análise empírica mostrou que grupos e subgrupos com no mínimo 5 e máximo de 10 itens são mais eficazes para definir o contexto, uma vez que são mais coesos do que grupos com grande número de itens.

A acurácia da tarefa de recomendação é avaliada por meio da medida $M A P$ (Mean Average Precision) em um protocolo de validação cruzada com 10 folds. Na Figura 6.3 é apresentada uma comparação entre o IBCF, Filter PoF ( $\alpha=0)$ e Filter PoF ( $\alpha=0.5)$, onde em $M A P-5$, a acurácia é computada com base em 5 recomendações e, em $M A P-10$, com base em 10 recomendações.

É possível notar que o uso de modelos de agrupamento como informação de contexto aumenta a acurácia da tarefa de recomendação. Em particular, quando os modelos de agrupamento são obtidos considerando tanto informação técnica quanto informação privilegida, o aumento da acurácia é ainda maior. Esse é um indicativo que as entidades nomeadas podem ser utilizadas, em determinados cenários, também como informação privilegiada.

\footnotetext{
${ }^{11}$ JPretext: http://sites.labic.icmc.usp.br/torch/msd2011/jpretext/

${ }^{12}$ REMBRANDT: http://xldb.di.fc.ul.pt/Rembrandt/
} 


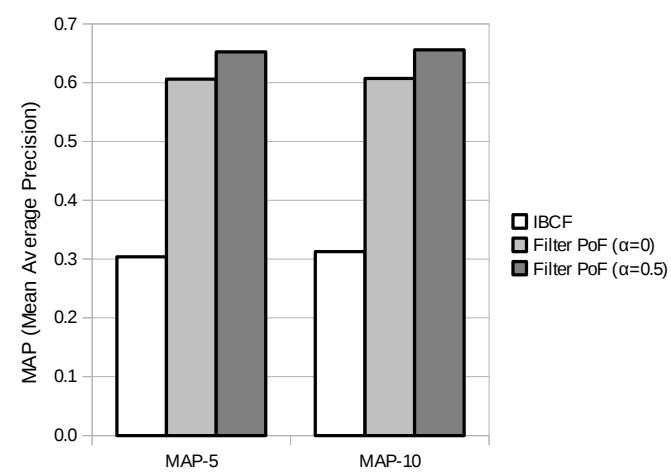

Figura 6.3: Comparação da acurácia do sistema recomendação entre IBCF (não utiliza contexto), Filter $\operatorname{PoF}(\alpha=0)$ que utiliza contexto sem informação privilegiada, e Filter PoF $(\alpha=0.5)$ que utiliza contexto baseado na informação privilegiada.

\subsection{Considerações Finais}

Neste capítulo foram apresentadas duas aplicações desenvolvidas durante este projeto, com objetivo de discutir o uso de informação privilegiada em tarefas de agrupamento para outros cenários. As aplicações envolveram colaboração com outros grupos de pesquisadores, permitindo discutir o trabalho realizado considerando outras perspectivas e linhas de pesquisa.

Os trabalhos discutidos neste capítulo foram publicados na forma de artigos em conferências e periódicos. Em relação à aplicação para Análise Exploratória de Mídias Sociais, as estratégias discutidas e desenvolvidas na ferramenta TorchSR foram publicadas no Brazilian Workshop on Social Network Analysis and Mining (BraSNAM) (Carvalho et al., 2012b). O trabalho foi convidado para ser estendido e publicado no periódico Social Networking (Carvalho et al., 2014). O estudo sobre refinamento do dendrograma para facilitar a visualização de agrupamentos hierárquicos foi publicado no ECML/PKDD 2012 Discovery Challenge (Marcacini et al., 2012a). Este último foi resultado de uma competição para solucionar problemas de grande escala envolvendo dados textuais. O trabalho discutido em Marcacini et al. (2012a) é resultante da única equipe a resolver o problema da trilha de aprendizado não supervisionado para grandes coleções textuais.

Já em relação à aplicação de Sistemas de Recomendação Sensiveis ao Contexto, o estudo sobre uso de entidades nomeadas como informação privilegiada em agrupamento hierárquico foi publicado no 18th International Database Engineering \& Applications Symposium (IDEAS) (Sinoara et al., 2014). Estudos sobre o uso de modelos de agrupamento como informação de contexto em 
sistemas de recomendação, considerando diferentes abordagens, foram publicados na 22nd International Conference on Pattern Recognition (ICPR) (Domingues et al., 2014b; Manzato et al., 2014). Por fim, estudos envolvendo avaliação de algoritmos de sistemas recomendação baseado em contexto, particularmente incoporando informação privilegiada via abordagem LIHC, foram publicados no Brazilian Conference on Intelligent System (Sundermann et al., 2014) e na IEEE/WIC/ACM International Conference on Web Intelligence (WI 2014) (Domingues et al., 2014a). 



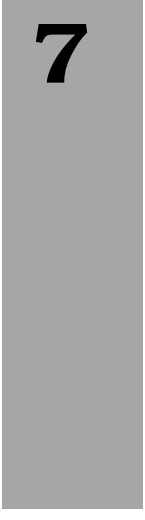

\section{Conclusões}

Neste capítulo são apresentadas as considerações finais deste trabalho de doutorado. Para tal, as questões de pesquisa definidas na introdução são retomadas e relacionadas com as contribuições científicas alcançadas durante o desenvolvimento do doutorado. Uma lista de artigos em conferências, periódicos e relatórios técnicos resultantes das contribuições científicas da tese também é apresentada. Por fim, são discutidas as limitações existentes nas abordagens propostas neste trabalho, bem como direções para trabalhos futuros.

\subsection{Contribuições Científicas}

A seguir, são discutidos os questionamentos relacionados aos objetivos deste trabalho, definidos na introdução, apresentando as possíveis soluções e as abordagens propostas.

1. Qual a importância da informação privilegiada para aprendizado não supervisionado de máquina? Como extrair informação privilegiada a partir de textos? Quais tipos de aplicações podem ser beneficiadas?

Documentos textuais são meios naturais para representar o conhecimento humano. Para representar esse tipo de dado em um formato estruturado, modelos baseado em bag-of-words são mais populares, devido à facilidade para extração e representação dos textos. O baixo custo computacional desta representação, bem como sua grande disponibilidade, tornam a bag-of-words um exemplo típico de informação técnica e o alvo do processo de aprendizado. 
No paradigma de aprendizado de máquina denominado LUPI (Learning Using Privileged Information) (Vapnik e Vashist, 2009) foi apresentada uma estratégia promissora para incorporar conhecimento adicional e específico de domínio (informação privilegiada) em algoritmos de aprendizado supervisionado de máquina. Neste trabalho de doutorado, o paradigma LUPI foi estendido para aprendizado não supervisionado, em particular, com foco em agrupamento hierárquico de textos.

A informação privilegiada se mostrou importante como uma nova e valiosa representação (adicional) dos textos, que complementa a informação técnica por meio de atributos (termos) específicos do domínio do problema. Por ser uma informação mais custosa e menos disponivel (geralmente disponível apenas para uma amostra dos textos), a informação privilegiada é empregada durante o aprendizado de um modelo inicial, permitindo refinar e corrigir o modelo de agrupamento. O modelo inicial é, então, utilizado para agrupar todo o conjunto de documentos.

Uma vez que a informação privilegiada é uma representação adicional, por meio de atributos (termos) específicos do domínio, buscou-se investigar técnicas para apoiar a extração de informação privilegiada a partir de textos. Com base em trabalhos relacionados, foi proposta uma abordagem para extração de informação privilegiada em textos. Tal proposta é implementada na ferramenta $\mathrm{AL}^{2} \mathrm{FIC}$ (Marcacini et al., 2012b), que utiliza conceitos de aprendizado ativo para que usuários (especialistas de domínio) possam selecionar um conjunto relevante de termos (Capítulo 3, Seção 3.4). A importância deste tipo de informação privilegiada para a qualidade dos modelos de agrupamento foi verificada em diferentes cenários: consenso de agrupamentos, aprendizado de métricas e seleção de modelos. Em cada um desses três cenários foram propostas abordagens para incorporar informação privilegiada em modelos de agrupamento: LIHC (Capítulo 3, Seção 3.5), baseada em consenso de agrupamentos; IPIML-GLOBAL e IPIML-LOCAL (Capítulo 4), baseadas em aprendizado de métricas; cdi-MAX e edi-MAX (Capítulo 5), baseadas em seleção de modelos. Em todos os cenários explorados, foi possível observar um aumento significativo da acurácia do modelo de agrupamento gerado.

Além dos aspectos teóricos relacionados à incorporação de informação privilegiada em modelos de agrupamento, também foram exploradas algumas aplicações que podem se beneficiar das abordagens aqui propostas (Capítulo 6). Em particular, o paradigma LUPI é potencialmente interessante para aplicações envolvendo análise exploratória de textos. A organização de documen- 
tos em grupos e subgrupos permitem que usuários explorem o conhecimento embutido nos dados textuais em diversos niveis de granularidade. A informação privilegiada permite obter modelos de agrupamentos com maior qualidade, uma vez que considera informação específica do domínio da aplicação para geração dos grupos. Ainda, para facilitar a interpretação do modelo de agrupamento, os termos da informação privilegiada podem ser utilizados como descritores dos grupos. Um exemplo desse tipo de aplicação foi explorado durante o doutorado, envolvendo a análise exploratória de mídias sociais (Capítulo 6, Seção 6.2).

Em geral, aplicações que utilizam agrupamento dos textos como uma das entradas de dados também podem ser beneficiadas. Para exemplificar esse tipo de aplicação, nesse trabalho foi investigado modelos de agrupamento obtidos com o LIHC para aplicações baseadas em sistemas de recomendação sensiveis ao contexto. Nesse caso, os agrupamentos obtidos com informação privilegiada foram empregados para refinar as recomendações sugeridas (boletins de agronegócios) aos usuários de um portal web.

\section{Como combinar, em um único modelo de agrupamento, os diferentes modelos provenientes das representações de informação técnica e infor- mação privilegada? Qual o ganho que a incorporação de informação privilegiada proporciona para a etapa de agrupamento incremental? Qual a importância de cada representação para o modelo final de agru- pamento?}

Consenso de agrupamentos foi investigado como uma estratégia interessante para incorporar informação privilegiada em agrupamento de textos. A ideia básica é obter diversas soluções a partir da representação textual de informação técnica, com uso de algoritmos de agrupamento e métricas apropriadas para esta representação. Da mesma forma, é possível obter diversas soluções de agrupamento a partir da representação textual de informação privilegiada. Nesse ponto, combinar as diferentes soluções em um modelo consensual é uma forma de combinar a informação técnica e informação privilegiada em uma solução final. Esta solução final é potencialmente mais robusta do que as soluções individuais, o que pode ser observado durante o processo de agrupamento incremental.

A abordagem LIHC foi proposta, neste trabalho de doutorado, para realizar o consenso de agrupamentos considerando o cenário LUPI (Marcacini et al., 2012c; Marcacini e Rezende, 2013). Para tal, as diferentes soluções de agrupamento são sumarizadas em uma matriz de coassociação, em que a simila- 
ridade entre um par de documentos é computada a partir da quantidade de vezes em que esses documentos são alocados no mesmo grupo, considerando as diferentes soluções. Uma das contribuições é a estratégia proposta para definir a importância de cada representação (técnica ou privilegiada) no consenso de agrupamentos. Para tal, foi proposto o Fator de Contribuição (Correa et al., 2014), um parâmetro que define o peso da informação privilegiada e o da informação técnica durante o cálculo da matriz de coassociação. Nas avaliações experimentais realizadas, observou-se que um valor equilibrado para o Fator de Contribuição leva a melhores resultados em relação à acurácia do modelo de agrupamento.

3. Como utilizar a informação privilegiada para aprender novas métricas? Qual o ganho para o agrupamento de textos no espaço de informação técnica ao utilizar as métricas aprendidas no espaço de informação privilegiada? Guais as vantagens e desvantagens entre empregar uma medida de proximidade global e várias medidas de proximidade locais?

Diversos algoritmos de agrupamento são baseados em uma medida de proximidade entre os exemplos. Dessa forma, é natural a investigação de quais medidas de proximidade são mais interessantes para determinados tipos de dados. Nesse trabalho, abordagens para aprendizado de métricas foram investigadas. A nova medida é aprendida considerando as relações de proximidade mais significativas no espaço de informação privilegiada. Assim, se dois documentos são muitos similares no espaço de informação privilegiada, é interessante que tal característica esteja presente também no espaço de informação técnica. Nesse sentido, foi proposta uma técnica para extrair automaticamente restrições do tipo Must-Link no espaço de informação privilegiada. Tais restrições foram empregadas durante um processo de aprendizado de métricas no espaço de informação técnica.

Duas abordagens de aprendizado de métricas foram propostas e avaliadas (Marcacini et al., 2014). A primeira, denominada IPIML-GLOBAL, extrai todas as restrições uma única vez, no início do processo, e as utilizam para o aprendizado de uma métrica global. Esta métrica é utilizada durante todo o processo de agrupamento. A segunda, denominada IPIML-LOCAL, extrai as restrições a cada nível do agrupamento hierárquico. No primeiro nível, a métrica aprendida é a mesma utilizada no IPIML-GLOBAL. Nos próximos níveis, as restrições são extraídas considerando apenas as relações entre os documentos de cada grupo. Foi observado que a abordagem para aprendizado de métricas locais permite eliminar possíveis restrições inválidas extraídas nos 
níveis superiores. Como consequência, o uso de métricas locais leva a melhores soluções de agrupamento. Além dessa vantagem, foi observado que o uso de métricas locais, especializadas para cada grupo, são mais eficazes durante e etapa de agrupamento incremental (reduzindo a degradação do modelo). Por outro lado, há a desvantagem em relação ao maior custo computacional envolvido no processo de aprendizado de várias métricas.

4. Por que a informação privilegiada é interessante para seleção de modelos de agrupamento obtidos a partir da informação técnica? Como selecionar modelos de agrupamento hierárquico com apoio de informação privilegiada? As informações sobre a estrutura topológica da hierarquia são importantes para a seleção de modelos?

Um dos desafios da área de análise de agrupamentos é selecionar um modelo de agrupamento adequado, dentre um conjunto de modelos. Tradicionalmente, critérios estatísticos que verificam o quão bem o modelo de agrupamento se ajusta ao conjunto de dados são empregados para esta tarefa. No entanto, esses critérios assumem que uma determinada medida de proximidade é adequada, ou que os dados possuem uma determinada distribuição; o que é difícil de definir em cenários práticos. Outra alternativa é utilizar critérios de validação externa, ou seja, que comparam o modelo de agrupamento com um modelo de referência. Por outro lado, em cenários reais, dificilmente há um modelo de referência dos dados.

A existência de uma representação textual adicional e dependente de domínio, como a informação privilegiada, permite uma nova abordagem para seleção de modelos. Considerando que a estrutura de agrupamento existente na informação privilegiada é mais próxima às expectativas dos usuários, o modelo de agrupamento proveniente da representação de informação privilegiada é utilizada como um modelo de referência. Dessa forma, dentre diversos modelos de agrupamento provenientes da informação técnica, é selecionado o modelo mais próximo ao de referência.

Uma vez que o uso de informação privilegiada se mostrou uma alternativa promissora para o desafio de seleção de modelos, neste trabalho de doutorado foram propostas duas abordagens: cdi-MAX (Maximum Cophenetic Difference Index) e edi-MAX (Maximum Edge Distance Index). A primeira utiliza tanto a topologia da hierarquia quanto o valor da fusão entre grupos e subgrupos durante a seleção de modelos. Já a segunda utiliza apenas a topologia da hierarquia para a seleção de modelos. As avaliações experimentais indicam que o uso das informações da hierarquia durante a seleção de modelos é mais pro- 
missor do que a abordagem aRi-MAX proposta por Feyereisl e Aickelin (2012), que compara a proximidade entre as partições durante a seleção de modelos (sem considerar informação da hierarquia). Em especial, a tarefa de seleção de modelos com uso de informação privilegiada é mais efetiva quando são considerados a topologia da árvore e valores de fusão de grupos e subgrupos, conforme proposto na abordagem cdi-MAX.

\subsection{Publicações Relacionadas}

As contribuições obtidas com o desenvolvimento desta tese de doutorado foram divulgadas por meio de publicações em conferências nacionais (4) e internacionais (9), periódicos (2) e relatório técnico (1). Uma lista dessas publicações (ordenadas pela data da publicação) é apresentada a seguir:

\section{Conferências Nacionais:}

Marcacini, R. M., Hruschka, E. R., e Rezende, S. O. (2012). On the use of consensus clustering for incremental learning of topic hierarchies. In 21st Brazilian Symposium on Artificial Intelligence (SBIA/BRACIS), Advances in Artificial Intelligence (Springer), páginas 112-121, Curitiba, PR, Brazil. NÃ£o citado no texto.

Carvalho, D. B. F., Marcacini, R. M., Lucena, C. J. P., e Rezende, S. O. (2012). Towards a process to support solving the content selection problem from online community forums. In Brazilian Workshop on Social Network Analysis and Mining (BraSNAM), Curitiba, PR, Brazil. NÃ£o citado no texto.

Rossi, R. G., Marcacini, R. M., e Rezende, S. O. (2013). Analysis of statistical keyword extraction methods for incremental clustering. In $X$ Encontro Nacional de Inteligência Artificial e Computacional (ENIAC), Fortaleza, CE, Brazil. NÃ£o citado no texto.

Sundermann, C. V., Domingues, M. A., Marcacini, R. M., e Rezende, S. O. (2014). Using topic hierarchies with privileged information to improve context-aware recommender systems. In Brazilian Conference on Intelligent System (BRACIS), São Carlos, SP, Brazil. NÃ£o citado no texto.

\section{Conferências Internacionais:}

Marcacini, R. M., Correa, G. N., e Rezende, S. O. (2012b). An active learning approach to frequent itemset-based text clustering. In 21st International Conference on Pattern Recognition (ICPR), páginas 3529-3532, Tsukuba, Japan. NÃ£o citado no texto.

Marcacini, R. M., Cherman, E. A., Metz, J., e Rezende, S. O. (2012). A fast dendrogram refinement approach for unsupervised expansion of hierarchies. In ECML/PKDD 2012 Discovery Challenge: Third Challenge on Large Scale Hierarchical Text Classification, Bristol, UK. NÃ£o citado no texto.

Marcacini, R. M., Domingues, M. A., e Rezende, S. O. (2013). Improving consensus clustering of texts using interactive feature selection. In 22nd International Conference on World Wide Web Companion (WWW), páginas 237-238, Rio de Janeiro, RJ, Brazil. NÃ£o citado no texto. 
Marcacini, R. M. e Rezende, S. O. (2013). Incremental hierarchical text clustering with privileged information. In 13th ACM Symposium on Document Engineering (DocEng), páginas 231-232, Florence, Italy. NÃ£o citado no texto.

Marcacini, R. M., Domingues, M. A., Hruschka, E. R., e Oliveira, S. R. (2014). Privileged information for hierarchical document clustering: A metric learning approach. In 22nd International Conference on Pattern Recognition (ICPR), Stockholm, Sweden. NÃ£o citado no texto.

Sinoara, R. A., Sundermann, C. V., Marcacini, R. M., Domingues, M. A., e Rezende, S. O. (2014). Named entities as privileged information for hierarchical text clustering. In 18th International Database Engineering \& Applications Symposium (IDEAS), Porto, Portugal. NÃ£o citado no texto.

Domingues, M., Sundermann, C., Manzato, M., Marcacini, R. M., e Rezende, S. (2014). Exploiting text mining techniques for contextual recommendations. In IEEE/WIC/ACM International Conference on Web Intelligence (WI 2014), Warsaw, Poland. NÃ£o citado no texto.

Domingues, M. A., Manzato, M., Marcacini, R. M., Sundermann, C. V., e Oliveira, S. R. (2014). Using contextual information from topic hierarchies to improve context-aware recommender systems. In 22nd International Conference on Pattern Recognition (ICPR), Stockholm, Sweden. NÃ£o citado no texto.

Manzato, M., Domingues, M. A., Marcacini, R. M., e Oliveira, S. R. (2014). Improving personalized ranking in recommender systems with topic hierarchies and implicit feedback. In 22nd International Conference on Pattern Recognition (ICPR), Stockholm, Sweden. NÃ£o citado no texto.

\section{Periódicos:}

Bonin, R., Marcacini, R. M., e Rezende, S. O. (2014). Unsupervised instance selection from text streams. Journal of Information and data Management, 5(1):114-123. NÃ£o citado no texto.

Carvalho, D., Marcacini, R. M., Lucena, C., e Rezende, S. (2014). A Process to Support Analysts in Exploring and Selecting Content from Online Forums. Social Networking, 3(2):86-93. NÃ£o citado no texto.

\section{Relatório Técnico:}

Rossi, R. G., Marcacini, R. M., e Rezende, S. O. (2013). Benchmarking text collections for classification and clustering tasks. Technical Report 395, Institute of Mathematics and Computer Sciences - University of São Paulo. NÃ£o citado no texto.

Além das publicações apresentadas acima, os seguintes trabalhos previstos para publicação em estão em fase de elaboração e/ou processo de revisão:

Marcacini, R. M., Hruschka, E. R., e Rezende, S. O. (2014b). Unsupervised learning using privileged information. Information Processing \& Management. In preparation. NÃ£o citado no texto.

Correa, G. N., Marcacini, R. M., Hruschka, E. R., e Rezende, S. O. (2014). Interactive textual feature selection for consensus clustering. Pattern Recognition Letters. In review. NÃ£o citado no texto.

Rossi, R. G., Marcacini, R. M., e Rezende, S. O. (2014). Analysis of statistical keyword extraction methods for incremental clustering. Learning Nonlinear Models (L\&NLM). In review. NÃ£o citado no texto. 


\subsection{Limitações e Trabalhos Futuros}

Durante o desenvolvimento deste trabalho, a representação textual de informação privilegiada foi constituída de termos específicos de domínio extraídos por um processo de simulação de usuários, com apoio da ferramenta $\mathrm{AL}^{2}$ FIC. As avaliações experimentais realizadas (em cenários controlados) utilizaram apenas este tipo de informação privilegiada. Embora esta representação seja adequada para aprendizado de máquina com base no paradigma LUPI, existem diferentes técnicas para pré-processamento de textos que podem ser utilizadas, como as entidades nomeadas brevemente investigadas nas aplicações envolvendo sistemas de recomendação sensíveis ao contexto. Nesse sentido, uma direção para trabalho futuro é explorar técnicas avançadas de pré-processamento de textos para extração de outras representações de informação privilegiada, principalmente àquelas que permitem inclusão de conhecimento de domínio, como representações enriquecidas com dados da Wikipedia e da DBPedia, uso de ontologias e ferramentas de anotação colaborativa.

Em relação ao uso de consenso de agrupamentos para incorporar informação privilegiada, as diferentes partições dos dados foram obtidas por diversas execuções do algoritmo k-means. Esta escolha é fundamentada em trabalhos anteriores na área, que indicam que a combinação de agrupadores simples (ou fracos) permite obter uma solução robusta. No entanto, também é reconhecido que a diversidade de soluções de agrupamento individuais é um requisito importante em consenso de agrupamentos. Uma forma de contornar esta limitação é empregar diferentes tipos de algoritmos de agrupamento nesta etapa. Uma direção para trabalhos futuros é utilizar a informação privilegiada visando selecionar modelos de agrupamento de forma a garantir a diversidade do conjunto.

As abordagens que incorporam informação privilegiada para aprendizado de métricas foram baseadas em restrições do tipo Must-Link, automaticamente extraídas da representação de informação privilegiada. Esta decisão foi baseada no fato de que um grande conjunto de restrições Cannot-Link pode levar à intratabilidade do problema. Para contornar esta limitação, é possível recorrer a técnicas para remover restrições ruidosas, redundantes ou conflituosas. Incorporar essas técnicas durante a extração de restrições a partir da informação privilegiada é uma direção para trabalhos futuros.

Já em relação às abordagens que utilizam informação privilegiada para seleção de modelos, é possível observar que a informação privilegiada não é 
incorporada (de fato) no modelo inicial de agrupamento. Neste caso, a informação privilegiada é limitada a atuar apenas como modelos de referência. Uma forma para contornar esta limitação é combinar os dois modelos de agrupamento obtidos na etapa seleção. Desse modo, o modelo de referência proveniente da informação privilegiada e o modelo proveniente da informação técnica podem ser empregados para gerar um terceiro modelo. Para tal, é possível utilizar uma matriz de coassociação, a exemplo do consenso de agrupamentos, para identificar documentos que estão alocados nos mesmos (sub)grupos das duas hierarquias. Esta é uma direção de trabalho futuro em relação à tarefas de agrupamento com base em seleção de modelos com uso de informação privilegiada.

Finalmente, é importante comentar sobre a estratégia de agrupamento hierárquico incremental. Neste projeto de doutorado foi utilizado uma estratégia tradicional da literatura para a etapa on-line, baseada na técnica de vizinho mais próximo. Uma vez obtido o modelo de agrupamento inicial, a mesma estrutura de agrupamento hierárquico é utilizada durante todo o processo de agrupamento incremental. No entanto, novos (sub)grupos podem ser criados, unidos ou removidos com base em novos documentos inseridos no modelo. Desse modo, uma outra direção para trabalhos futuros é investigar técnicas de atualização dinâmica do modelo de agrupamento na etapa on-line, visando reduzir a degradação do modelo conforme o tempo. 



\section{Referências Bibliográficas}

Abney, S. (2002). Bootstrapping. In Annual Meeting on Association for Computational Linguistics (ACL), páginas 360-367, Stroudsburg, PA, USA. Association for Computational Linguistics. http://dx.doi.org/10.3115/ 1073083.1073143 . Citado na página 31.

Adomavicius, G., Sankaranarayanan, R., Sen, S., e Tuzhilin, A. (2005). Incorporating contextual information in recommender systems using a multidimensional approach. ACM Transactions on Information Systems (TOIS), 23(1):103-145. http://doi.acm.org/10.1145/1055709.1055714. Citado na página 123.

Adomavicius, G. e Tuzhilin, A. (2011). Context-aware recommender systems. In Ricci, F., Rokach, L., Shapira, B., e Kantor, P. B., editors, Recommender Systems Handbook, páginas 217-253. Springer US. http://dx.doi.org/ 10.1007/978-0-387-85820-3_7. Citado na página 123.

Agarwal, R. e Srikant, R. (1994). Fast algorithms for mining association rules. In 20th Very Large Data Bases Conference (VLDB), páginas 487-499. http: //dl.acm.org/citation.cfm?id=672836. Citado nas páginas 51 e 56.

Aggarwal, C. e Zhao, P. (2013). Towards graphical models for text processing. Knowledge and Information Systems, 36(1):1-21. http:// dx . doi . org/10 . 1007/s10115-012-0552-3. Citado nas páginas 44 e 49.

Aggarwal, C. C. e Reddy, C. K. (2013). An Introduction to Cluster Analysis. Chapman \& Hall/CRC, Data Mining and Knowledge Discovery Series. http://www.crcpress.com/product/isbn/9781466558212. Citado nas páginas $3,14,17,20,21$, e 95 .

Aggarwal, C. C. e Zhai, C. X. (2012a). Mining Text Data. Springer Publishing Company. http://dl.acm.org/citation.cfm?id=2331533. Citado nas páginas $2,15,22$, e 43. 
Aggarwal, C. C. e Zhai, C. X. (2012b). A survey of text clustering algorithms. In Aggarwal, C. C. e Zhai, C. X., editors, Mining Text Data, páginas 77128. Springer US. http://dx.doi.org/10.1007/978-1-4614-3223-4_4. Citado nas páginas 1 e 15.

Allan, J. (2002). Introduction to topic detection and tracking. In Allan, J., editor, Topic Detection and Tracking, volume 12 of The Information Retrieval Series, páginas 1-16. Springer US. http://dx.doi.org/10.1007/ 978-1-4615-0933-2_1. Citado na página 28.

Anastasiu, D. C., Tagarelli, A., e Karypis, G. (2013). Document clustering: The next frontier. In Aggarwal, C. C. e Reddy, C. K., editors, Data Clustering: Algorithms and Applications, páginas 305-328. Chapman and Hall/CRC. http://dx.doi.org/10.1201/b15410-14. Citado na página 1.

Baghshah, M. S. e Shouraki, S. B. (2009). Semi-supervised metric learning using pairwise constraints. In International Joint Conference on Artificial Intelligence (IJCAI), volume 9, páginas 1217-1222. http://ijcai.org/ papers09/Papers/IJCAI09-205.pdf. Citado na página 73.

Basu, S., Davidson, I., e Wagstaff, K. (2008). Constrained Clustering: Advances in Algorithms, Theory, and Applications. Chapman \& Hall/CRC, $1^{\text {a }}$ edição. http://dl.acm.org/citation.cfm?id=1404506. Citado nas páginas 13 e 25.

Bechet, F. (2011). Named Entity Recognition, páginas 257-290. John Wiley \& Sons, Ltd. http://dx.doi.org/10.1002/9781119992691.ch10. Citado na página 2 .

Beil, F., Ester, M., e Xu, X. (2002). Frequent term-based text clustering. In International Conference on Knowledge Discovery and Data Mining (SIGKDD), páginas 436-442. ACM. http://dx.doi.org/10.1145/775047.775110. Citado na página 50.

Bekkerman, R. e Allan, J. (2004). Using bigrams in text categorization. Relatório Técnico IR-408, Center of Intelligent Information Retrieval, UMass Amherst. http://ciir.cs.umass.edu/pubfiles/ir-408.pdf. Citado na página 47.

Bickel, S. e Scheffer, T. (2004). Multi-View Clustering. In IEEE International Conference on Data Mining (ICDM), páginas 19-26, Washington, DC, USA. IEEE Computer Society. http: / / dx. doi .org/10.1109/ ICDM. 2004.10095. Citado nas páginas $2,23,24$, e 31 .

Bilenko, M., Basu, S., e Mooney, R. J. (2004). Integrating constraints and metric learning in semi-supervised clustering. In 21st International Conference on Machine Learning (ICML), página 11, New York, NY, USA. ACM. http://dx.doi.org/10.1145/1015330.1015360. Citado nas páginas 3, $25,27,73,79$, e 80 . 
Bishop, C. M. (2007). Pattern Recognition and Machine Learning (Information Science and Statistics). Springer-Verlag New York, Inc., Secaucus, NJ, USA. Citado na página 13.

Blitzer, J., Weinberger, K. Q., e Saul, L. K. (2005). Distance metric learning for large margin nearest neighbor classification. In Advances in Neural Information Processing Systems (NIPS), páginas 1473-1480. http: //books.nips.cc/papers/files/nips18/NIPS2005_0265.pdf. Citado na página 80 .

Blum, A. e Mitchell, T. (1998). Combining labeled and unlabeled data with cotraining. In Conference on Computational Learning Theory (COLT), páginas 92-100, New York, NY, USA. ACM. http://dx.doi.org/10.1145/279943. 279962. Citado na página 31.

Bobadilla, J., Ortega, F., Hernando, A., e Gutiarrez, A. (2013). Recommender systems survey. Knowledge-Based Systems, 46(0):109 - 132. http://dx. doi.org/10.1016/j.knosys.2013.03.012. Citado na página 123.

Bonin, R., Marcacini, R. M., e Rezende, S. O. (2014). Unsupervised instance selection from text streams. Journal of Information and data Management, 5(1):114-123. https://seer.lcc.ufmg.br/index.php/jidm/ article/view/287. Citado nas páginas 10, 61, e 72.

Bouchachia, A. (2008). Incremental learning. In Encyclopedia of Data Warehousing and Mining, páginas 1006-1012. IGI Global. http://dx.doi. org/10.4018/978-1-60566-010-3.ch156. Citado na página 3 .

Cai, F. e Cherkassky, V. (2012). Generalized smo algorithm for svmbased multitask learning. IEEE Transactions on Neural Networks and Learning Systems, 23(6):997-1003. http://dx.doi.org/10.1109/TNNLS. 2012.2187307. Citado nas páginas 36 e 41.

Calinski, T. e Harabasz, J. (1974). A dendrite method for cluster analysis. Communications in Statistics, 3(1):1-27. http://dx.doi.org/10.1080/ 03610927408827101 . Citado na página 98.

Campello, R. J. G. B. e Hruschka, E. R. (2009). On comparing two sequences of numbers and its applications to clustering analysis. Information Sciences, 179(8):1025 - 1039. http://dx.doi.org/10.1016/j.ins.2008.11.028. Citado na página 104.

Carvalho, A. L. C., Moura, E. S., e Calado, P. (2010). Using statistical features to find phrasal terms in text collections. Journal of Information and Data Management, 1(3):583-597. https:// seer.lcc.ufmg.br/index.php/jidm/ article/view/79. Citado nas páginas 43 e 49.

Carvalho, D., Madeira, W., Okamura, M., Lucena, C., e Zanetta, S. (2012a). A practical approach to exploit public data available on the internet to study healthcare issues. In XII Workshop on Medical Informatics at XXXII Congress 
of the Brazilian Computer Society Computer Society. http: / / www . 1bd. dcc. ufmg.br/bdbcomp/servlet/Trabalho?id=11750. Citado na página 118.

Carvalho, D., Marcacini, R., Lucena, C., e Rezende, S. (2014). A Process to Support Analysts in Exploring and Selecting Content from Online Forums. Social Networking, 3(2):86-93. http://dx.doi.org/10.4236/sn. 2014.32011. Citado nas páginas 10, 119, e 128.

Carvalho, D. B. F. (2013). Combining a process and tools to support the analysis of online communities applied to healthcare. Tese de Doutorado. Pontífica Universidade Católica do Rio de Janeiro (PUC-RIO). "http://www2.dbd.puc-rio.br/pergamum/tesesabertas/0921317_ 2013_pretextual.pof". Citado na página 122.

Carvalho, D. B. F., Marcacini, R. M., Lucena, C. J. P., e Rezende, S. O. (2012b). Towards a process to support solving the content selection problem from online community forums. In Brazilian Workshop on Social Network Analysis and Mining (BraSNAM), páginas 1-4, Curitiba, PR, Brazil. http://www.lbd.dcc.ufmg.br/colecoes/brasnam/2012/0028.pdf. Citado nas páginas 10, 119, e 128.

Carvalho, V. R. e Cohen, W. W. (2006). Improving "email speech acts" analysis via n-gram selection. In Proceedings of the Workshop on Analyzing Conversations in Text and Speech (ACTS), páginas 35-41, Morristown, NJ, USA. Association for Computational Linguistics. http: / /www . aclweb. org/ anthology/W/W06/W06-34.pdf. Citado na página 48.

Chapelle, O., Schlkopf, B., e Zien, A. (2010). Semi-Supervised Learning. The

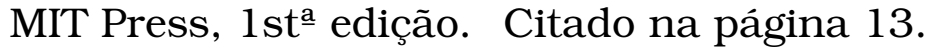

Correa, G. N., Marcacini, R. M., Hruschka, E. R., e Rezende, S. O. (2014). Interactive textual feature selection for consensus clustering. Pattern Recognition Letters. In review. Citado nas páginas 9, 72, e 134.

Cortes, C. e Vapnik, V. (1995). Support-vector networks. Machine Learning, 20(3):273-297. http://dx.doi.org/10.1023/A:1022627411411. Citado na página 5.

Covões, T. F., Hruschka, E. R., e Ghosh, J. (2013). A study of k-means-based algorithms for constrained clustering. Intelligent Data Analysis, 17(3):485505. http://dx.doi.org/10.3233/IDA-130590. Citado nas páginas 3, $26,27,80$, e 88 .

Cutting, D. R., Karger, D. R., Pedersen, J. O., e Tukey, J. W. (1992). Scatter/gather: a cluster-based approach to browsing large document collections. In SIGIR'92: 15th International Conference on Research and Development in Information Retrieval, páginas 318-329. Citado na página 22.

Dai, X. Y., Chen, Q. C., Wang, X. L., e Xu, J. (2010). Online topic detection and tracking of financial news based on hierarchical clustering. In International 
Conference on Machine Learning and Cybernetics (ICMLC), volume 6, páginas 3341-3346. http://10.1109/ICMLC.2010.5580677. Citado na página 28.

Dasgupta, S., Littman, M., e McAllester, D. (2001). PAC generalization bounds for co-training. In NIPS'O1: Neural Information Processing Systems. Citado na página 31 .

Davidson, I. e Ravi, S. S. (2005). Clustering with constraints: Feasibility issues and the k-means algorithm. In 5th SIAM Data Mining Conference, páginas 138-149. http://dx.doi.org/10.1137/1.9781611972757.13. Citado nas páginas 25 e 26.

Davies, D. L. e Bouldin, D. W. (1979). A cluster separation measure. IEEE Transactions on Pattern Analysis and Machine Intelligence, PAMI-1(2):224227. http://dx.doi.org/10.1109/TPAMI.1979.4766909. Citado na página 98.

Demšar, J. (2006). Statistical comparisons of classifiers over multiple data sets. The Journal of Machine Learning Research, 7:1-30. http://dl . acm. org/citation.cfm?id=1248547.1248548. Citado nas páginas 70, 92, e 114 .

Deshpande, M. e Karypis, G. (2004). Item-based top-n recommendation algorithms. ACM Transactions on Information Systems (TOIS), 22(1):143-177. http://doi.acm.org/10.1145/963770.963776. Citado na página 125.

Dey, A. K. (2001). Understanding and using context. Personal Ubiquitous Computing, 5(1):4-7. http://dx.doi.org/10.1007/s007790170019. Citado na página 123.

Domingues, M., Sundermann, C., Manzato, M., Marcacini, R., e Rezende, S. (2014a). Exploiting text mining techniques for contextual recommendations. In IEEE/WIC/ACM International Conference on Web Intelligence (WI 2014), Warsaw, Poland. Citado nas páginas 10 e 129.

Domingues, M. A., Manzato, M., Marcacini, R. M., Sundermann, C. V., e Oliveira, S. R. (2014b). Using contextual information from topic hierarchies to improve context-aware recommender systems. In 22nd International Conference on Pattern Recognition (ICPR), páginas 1-6, Stockholm, Sweden. No prelo. Citado nas páginas 10 e 129.

Duda, R. O., Hart, P. E., e Stork, D. G. (2000). Pattern Classification (2Nd Edition). Wiley-Interscience. Citado na página 13.

Dunn, J. C. (1974). Well-separated clusters and optimal fuzzy partitions. Journal of Cybernetics, 4(1):95-104. http://dx.doi .org/10.1080/ 01969727408546059 . Citado na página 98.

Eaton, E., desJardins, M., e Jacob, S. (2010). Multi-view clustering with constraint propagation for learning with an incomplete mapping between 
views. In 19th ACM International Conference on Information and Knowledge Management (CIKM), páginas 389-398, New York, NY, USA. ACM. http: //doi.acm.org/10.1145/1871437.1871489. Citado na página 23.

Ekbal, A., Saha, S., e Sikdar, U. (2014). On active annotation for named entity recognition. International Journal of Machine Learning and Cybernetics, páginas 1-18. http://dx.doi.org/10.1007/s13042-014-0275-8. Citado na página 124.

Ekstrand, M. D., Riedl, J. T., e Konstan, J. A. (2011). Collaborative filtering recommender systems. Foundations and Trends in Human-Computer Interaction, 4(2):81-173. http://dx.doi.org/10.1561/1100000009. Citado na página 123.

Everitt, B. S., Landau, S., e Leese, M. (2001). Cluster Analysis. Arnold Publishers. Citado nas páginas 14, 16, 17, 95, 96, e 97.

Faceli, K., Carvalho, A. C. P. L. F., e Souto, M. C. P. (2005). Validação de algoritmos de agrupamento. Relatório Técnico 254, Instituto de Ciências Matemáticas e de Computação - ICMC - USP. http://dedalus.usp.br/F/? func= full-set-set\&set_number $=032693 \&$ set_entry $=000005$. Citado nas páginas 29, 95, e 99 .

Falkenauer, E. (1998). Genetic Algorithms and Grouping Problems. John Wiley \& Sons, Inc., New York, NY, USA. Citado na página 17.

Feldman, R. e Sanger, J. (2006). The Text Mining Handbook: Advanced Approaches in Analyzing Unstructured Data. Cambridge University Press. http://dx.doi.org/10.1017/CB09780511546914. Citado na página 46.

Feng Cai (2011). Advanced Learning Approaches Based on SVM+ Methodology. Tese de Doutorado. University of Minnesota. Disponível em: http://conservancy.umn.edu/bitstream/112973/1/Cai_umn_ 0130E_12047.pdf. Citado na página 36.

Feyereisl, J. e Aickelin, U. (2012). Privileged information for data clustering. Information Sciences, 194(0):4 - 23. http://dx.doi.org/10.1016/j.ins. 2011.04.025. Citado nas páginas 5, 6, 9, 37, 41, 96, 102, 108, e 136.

Finch, T. (2009). Incremental calculation of weighted mean and variance. University of Cambridge Computing Service. http://www-uxsup.csx.cam.ac. uk/ fanf2/hermes/doc/antiforgery/stats.pdf. Citado na página 28.

Flach, P. (2012). Machine Learning: The Art and Science of Algorithms That Make Sense of Data. Cambridge University Press, New York, NY, USA. Citado nas páginas 13 e 14 .

Floudas, C. C. A. e Pardalos, P. M. (2006). Encyclopedia of Optimization. Springer-Verlag, Secaucus, NJ, USA. Citado na página 77. 
Forman, G. (2003). An extensive empirical study of feature selection metrics for text classification. Journal of Machine Learning Research, 3:12891305. http://jmlr.org/papers/volume3/forman03a/forman03a_full. pdf. Citado nas páginas 47 e 48.

Fouad, S. (2013). Metric learning for incorporating privileged information in prototype-based models. Tese de Doutorado, University of Birmingham. http://etheses.bham.ac.uk/4615/. Citado na página 77 .

Fouad, S. e Tino, P. (2013). Ordinal-based metric learning for learning using privileged information. In International Joint Conference on Neural Networks (IJCNN), páginas 1-8. http://dx.doi.org/10.1109/I JCNN. 2013.6706799. Citado nas páginas 2 e 78.

Fouad, S., Tino, P., Raychaudhury, S., e Schneider, P. (2012). Learning using privileged information in prototype based models. In Villa, A. E. P., Duch, W., Erdi, P., Masulli, F., e Palm, G., editors, Artificial Neural Networks and Machine Learning (ICANN 2012), volume 7553 of Lecture Notes in Computer Science, páginas 322-329. Springer. http://dx.doi .org/10.1007/ 978-3-642-33266-1_40. Citado nas páginas 38 e 41.

Fouad, S., Tino, P., Raychaudhury, S., e Schneider, P. (2013). Incorporating privileged information through metric learning. IEEE Transactions on Neural Networks and Learning Systems, 24(7):1086-1098. http://dx.doi.org/ 10.1109/TNNLS.2013.2251470. Citado nas páginas 2, 38, 41, 74, e 78 .

Fowlkes, E. B. e Mallows, C. L. (1983). A method for comparing two hierarchical clusterings. Journal of the American Statistical Association, 78(383):553569. http://dx.doi.org/10.1080/01621459.1983.10478008. Citado na página 99.

Fraley, C. e Raftery, A. E. (1998). How many clusters? which clustering method? answers via model-based cluster analysis. The computer journal, 41(8):578-588. http://dx.doi.org/10.1093/comjnl/41.8.578. Citado na página 97.

Fung, B. C. M., Wang, K., e Ester, M. (2003). Hierarchical document clustering using frequent itemsets. In SIAM SDM'03: Proceedings of the International Conference on Data Mining, páginas 59-70. http://epubs . siam.org/doi/ abs/10.1137/1.9781611972733.6. Citado na página 50.

Fürnkranz, J. (1998). A study using n-gram features for text categorization. Relatório Técnico OEFAI-TR-98-30, Austrian Research Institute for Artificial Intelligence. http://www. ofai.at/cgi-bin/tr-online?number+98-30. Citado na página 47.

Garber, D. e Hazan, E. (2011). Approximating semidefinite programs in sublinear time. In Advances in Neural Information Processing Systems (NIPS), páginas 1080-1088. http://books.nips.cc/papers/files/ nips24/NIPS2011_0658.pdf. Citado na página 79. 
Garcia, R. G. e Porrata, A. P. (2010). Dynamic hierarchical algorithms for document clustering. Pattern Recognition Letters, 31(6):469 - 477. Citado na página 3.

Ghosh, J. e Acharya, A. (2011). Cluster ensembles. Wiley Interdisciplinary Reviews: Data Mining and Knowledge Discovery, 1(4):305-315. http://dx . doi.org/10.1002/widm.32. Citado nas páginas 23 e 24.

Giraud, C. (2000). A note on the utility of incremental learning. AI Communications, 13:215-223. Citado na página 27.

Gower, J. C. (1971). A general coefficient of similarity and some of its properties. Biometrics, 27(4):857-871. http: / /www. jstor.org/stable/2528823. Citado na página 15.

Grossman, D. A. e Frieder, O. (2004). Information Retrieval: Algorithms and Heuristics. Springer, Secaucus, NJ, USA. Citado na página 22.

Günnemann, S., Färber, I., e Seidl, T. (2012). Multi-view clustering using mixture models in subspace projections. In Proceedings of the 18th ACM SIGKDD international conference on Knowledge discovery and data mining, KDD '12, páginas 132-140, New York, NY, USA. ACM. Citado na página 2.

Halkidi, M., Batistakis, Y., e Vazirgiannis, M. (2001). On clustering validation techniques. Journal of Intelligent Information Systems, 17(2-3):107-145. http://dx.doi.org/10.1023/A:1012801612483. Citado nas páginas 29, 95, e 98 .

Hammouda, K. M. e Kamel, M. S. (2003). Incremental document clustering using cluster similarity histograms. In WI '03: Proceedings of the 2003 IEEE International Conference on Web Intelligence, página 597, Washington, DC, USA. IEEE Computer Society. Citado na página 28.

Hartigan, J. (1975). Clustering algorithms. Wiley New York. Citado na página 28.

Hastie, T., Tibshirani, R., e Friedman, J. (2009). Unsupervised learning. In The Elements of Statistical Learning, Springer Series in Statistics, páginas 485-585. Springer New York. http://dx.doi.org/10.1007/ 978-0-387-84858-7_14. Citado nas páginas 1 e 15.

Hauberg, S., Freifeld, O., e Black, M. (2012). A geometric take on metric learning. In Advances in Neural Information Processing Systems (NIPS), páginas 2033-2041. http://books.nips.cc/papers/files/nips25/NIPS2012_ 0997 .pdf. Citado na página 80.

Hu, Y., Milios, E. E., e Blustein, J. (2011). Interactive feature selection for document clustering. In ACM Symposium on Applied Computing (SAC), páginas 1143-1150, New York, NY, USA. ACM. http://doi .acm.org/10.1145/ 1982185.1982436. Citado nas páginas 53, 54, 63, e 96. 
Hu, Y., Milios, E. E., e Blustein, J. (2012). Enhancing semi-supervised document clustering with feature supervision. In Proceedings of the 27th Annual ACM Symposium on Applied Computing, SAC '12, páginas 929-936, New York, NY, USA. ACM. http://doi.acm.org/10.1145/2245276.2245457. Citado na página 55.

Hubert, L. e Arabie, P. (1985). Comparing partitions. Journal of classification, 2(1):193-218. Citado nas páginas 37, 96, 99, e 100.

Hussain, S., Mushtaq, M., e Halim, Z. (2014). Multi-view document clustering via ensemble method. Journal of Intelligent Information Systems, 43(1):8199. http://dx.doi.org/10.1007/s10844-014-0307-6. Citado na página 23.

Instituto-Sírio-Libanês (2012). Motivações para o início e o fim do consumo de drogas: Uma análise das comunidades virtuais relacionadas a cocaína, crack e maconha. Instituto de Responsabilidade Social Sírio-Libanês. Acessado em 10-07-2014. Notas não publicadas. http://goo.gl/8T6W8i. Citado na página 118.

Jain, A. K. (2010). Data clustering: 50 years beyond k-means. Pattern Recognition Letters, 31(8):651-666. http: / / dx. doi .org/10.1016/j.patrec. 2009.09.011. Citado nas páginas 22 e 59.

Jain, A. K. e Dubes, R. C. (1988). Algorithms for clustering data. Prentice-Hall, Upper Saddle River, NJ, USA. Citado na página 15.

Jain, A. K., Murty, M. N., e Flynn, P. J. (1999). Data clustering: a review. ACM Computing Surveys, 31(3):264-323. http://dx.doi.org/10.1145/ 331499.331504 . Citado nas páginas 1, 14, 15, 28, e 95.

Jan Feyereisl (2010). On the importance and incorporation of additional knowledge in cluster analysis. Tese de Doutorado. University of Nottingham. Disponivel em: http://citeseerx.ist.psu.edu/viewdoc/ download?doi=10.1.1.308.731\&rep=rep1\&type=pdf. Citado na página 37.

Japkowicz, N. e Shah, M. (2011). Evaluating learning algorithms: a classification perspective. Cambridge University Press. http://dx.doi.org/10 . 1017 /CBO9780511921803. Citado nas páginas 92, 100, e 114.

Jiang, J. (2012). Information extraction from text. In Mining Text Data, páginas 77-128. Springer-Verlag. Citado na página 2.

Joshi, S., Koyejo, O., e Ghosh, J. (2014). Constrained Inference for Multi-View Clustering. In Proceedings of the ICML Workshop on Multiview Clustering. Citado nas páginas 2 e 23. 
Ke, W., Sugimoto, C. R., e Mostafa, J. (2009). Dynamicity vs. effectiveness: studying online clustering for scatter/gather. In SIGIR'09: 32nd International Conference on Research and Development in Information Retrieval, páginas 19-26. Citado na página 22.

Kim, Y. M., Amini, M. R., Goutte, C., e Gallinari, P. (2010). Multi-view clustering of multilingual documents. In SIGIR'10: 33rd International Conference on Research and Development in Information Retrieval, páginas 821-822, New York, NY, USA. ACM. Citado na página 31.

Kohavi, R. (1995). A study of cross-validation and bootstrap for accuracy estimation and model selection. In International Joint Conference on Artificial Intelligence (IJCAI), volume 14, páginas 1137-1145. http: / / www. i jcai.org/ PastProceedings/IJCAI-95-VOL2/PDF/016.pdf. Citado na página 95.

Korenius, T., Laurikkala, J., e Juhola, M. (2007). On principal component analysis, cosine and euclidean measures in information retrieval. Information Sciences, 177(22):4893 - 4905. http://dx.doi.org/10.1016/j.ins. 2007.05 .027 . Citado na página 16.

Kozinets, R. V. (2010). Netnography: Doing Ethnographic Research Online. Sage Publications Ltd. http://books.google.com.br/books?id= QNDaeutR9v4C. Citado na página 118.

Kriegel, H. P. e Zimek, A. (2010). Subspace clustering, ensemble clustering, alternative clustering, multiview clustering: what can we learn from each other. In 1st International Workshop on Discovering, Summarizing and using Multiple Clusterings (MultiClust). Data Mining and Knowledge Discovery (KDD). Citado na página 23.

Kulis, B. (2013). Metric learning: A survey. Foundations and Trends in Machine Learning, 5(4):287 - 364. http://dx.doi.org/10.1561/ 2200000019 . Citado nas páginas 73, 78, e 80.

Lajugie, R., Bach, F., e Arlot, S. (2014). Large-margin metric learning for constrained partitioning problems. In The Journal of Machine Learning Research (JMLR), páginas 297-305. JMLR.org. http://jmlr.org/proceedings/ papers/v32/lajugie14.html. Citado na página 3.

Lan, M., Tan, C. L., Su, J., e Lu, Y. (2009). Supervised and traditional term weighting methods for automatic text categorization. IEEE Transactions on Pattern Analysis and Machine Intelligence, 31(4):721-735. http:/dx.doi . org/10.1109/TPAMI.2008.110. Citado na página 46.

Lange, T., Braun, M., Roth, V., e Buhmann, J. (2002). Stability-based model selection. páginas 617-624. http://books.nips.cc/papers/files/ nips15/AA17.pdf. Citado nas páginas 3, 100, e 101. 
Lange, T., Roth, V., Braun, M. L., e Buhmann, J. M. (2004). Stabilitybased validation of clustering solutions. Neural Computation, 16(6):12991323. http://dx.doi.org/10.1162/089976604773717621. Citado na página 100.

Lapin, M., Hein, M., e Schiele, B. (2014). Learning using privileged information: Svm+ and weighted $\{\mathrm{SVM}\}$. Neural Networks, 53(0):95 - 108. http://dx.doi.org/10.1016/j.neunet.2014.02.002. Citado nas páginas 4,39 , e 41 .

Larsen, B. e Aone, C. (1999). Fast and effective text mining using linear-time document clustering. In SIGKDD'99: Proceedings of the 5th ACM International Conference on Knowledge Discovery and Data Mining, páginas 16-22. Citado na página 29.

Lauer, F. e Bloch, G. (2008). Incorporating prior knowledge in support vector machines for classification: A review. Neurocomputing, 71(9):1578 1594. http://dx.doi.org/10.1016/j.neucom.2007.04.010. Citado na página 39.

Law, M. H., Topchy, A., e Jain, A. K. (2004). Clustering with soft and group constraints. In Joint IAPR International Workshops on Structural, Syntactic, And Statistical Pattern Recognition, páginas 662-670, Lisbon, Portugal. Citado na página 25.

Lawrie, D., Croft, W. B., e Rosenberg, A. (2001). Finding topic words for hierarchical summarization. In International ACM SIGIR Conference on Research and Development in Information Retrieval, páginas 349-357, New York, NY, USA. ACM. http://doi.acm.org/10.1145/383952.384022. Citado na página 119.

Lazer, D., Pentland, A., Adamic, L., Aral, S., Barabasi, A. L., Brewer, D., Christakis, N., Contractor, N., Fowler, J., Gutmann, M., Jebara, T., King, G., Macy, M., Roy, D., e Alstyne, M. V. (2009). Computational social science. Science, 323(5915):721-723. http://dx.doi.org/10.1126/ science.1167742. Citado na página 118.

Lebanon, G. (2006). Metric learning for text documents. Pattern Analysis and Machine Intelligence, IEEE Transactions on, 28(4):497-508. http:// dx.doi.org/10.1109/TPAMI.2006.77. Citado nas páginas 2, 73, e 78 .

Lefevre, F. e Lefevre, A. M. C. (2006). The Collective Subject That Speaks. Interface-Comunicaćão, Saúde, Educaćão, 10:517 - 524. http://dx.doi. org/10.1590/S1414-32832006000200017. Citado na página 118.

Li, Y., Chung, S. M., e Holt, J. D. (2008). Text document clustering based on frequent word meaning sequences. Data \& Knowledge Engineering, 64(1):381-404. http://dx.doi.org/10.1016/j.datak.2007.08.001. Citado na página 48. 
Li, Y., Nie, J., Zhang, Y., Wang, B., Yan, B., e Weng, F. (2010). Contextual recommendation based on text mining. In International Conference on Computational Linguistics, COLING '10, páginas 692-700, Stroudsburg, PA, USA. Association for Computational Linguistics. http://dl . acm.org/ citation. cfm?id=1944566.1944645. Citado na página 123.

Liu, H., Xiong, H., Chen, J., e Cao, J. (2014). K-means-based consensus clustering: A unified view. IEEE Transactions on Knowledge and Data Engineering, 99(1). http://doi.ieeecomputersociety.org/10.1109/TKDE. 2014.2316512. Citado na página 24.

Long, B., Yu, P., e Zhang, Z. (2008). A general model for multiple view unsupervised learning. In SDM'08: SIAM International Conference on Data Mining, páginas 822-833. Citado nas páginas 2, 24, e 31 .

MacQueen, J. B. (1967). Some methods for classification and analysis of multivariate observations. In Cam, L. M. L. e Neyman, J., editors, Proceedings of the 5th Berkeley Symposium on Mathematical Statistics and Probability, volume 1, páginas 281-297. University of California Press. Citado na página 17.

Maimon, O. e Rokach, L. (2005). Data Mining and Knowledge Discovery Handbook. Springer-Verlag, Secaucus, NJ, USA. Citado nas páginas 27 e 28.

Malik, H. H. e Kender, J. R. (2006). High quality, efficient hierarchical document clustering using closed interesting itemsets. International Conference on Data Mining (ICDM), 0:991-996. http://doi.ieeecomputersociety . org/10.1109/ICDM.2006.81. Citado na página 51.

Manning, C. D., Raghavan, P., e Schütze, H. (2008). An Introduction to Information Retrieval. Cambridge University Press. Citado nas páginas 2 e 120.

Manzato, M., Domingues, M. A., Marcacini, R. M., e Oliveira, S. R. (2014). Improving personalized ranking in recommender systems with topic hierarchies and implicit feedback. In 22nd International Conference on Pattern Recognition (ICPR), páginas 1-6, Stockholm, Sweden. No prelo. Citado nas páginas 10 e 129 .

Marcacini, R. M., Cherman, E. A., Metz, J., e Rezende, S. O. (2012a). A fast dendrogram refinement approach for unsupervised expansion of hierarchies. In ECML/PKDD 2012 Discovery Challenge: Third Challenge on Large Scale Hierarchical Text Classification, páginas 1-12, Bristol, UK. http:// lshtc.iit.demokritos.gr/system/files/lshc3_marcacini.pdf. Citado nas páginas 121 e 128 .

Marcacini, R. M., Correa, G. N., e Rezende, S. O. (2012b). An active learning approach to frequent itemset-based text clustering. In 21st International Conference on Pattern Recognition (ICPR), páginas 3529-3532, Tsukuba, Japan. http://ieeexplore.ieee.org/xpls/abs_all.jsp? arnumber $=6460926$. Citado nas páginas 8, 44, 55, 71, e 132 . 
Marcacini, R. M., Domingues, M. A., Hruschka, E. R., e Oliveira, S. R. (2014). Privileged information for hierarchical document clustering: A metric learning approach. In 22nd International Conference on Pattern Recognition (ICPR), Stockholm, Sweden. Citado nas páginas 9 e 134.

Marcacini, R. M., Domingues, M. A., e Rezende, S. O. (2013). Improving consensus clustering of texts using interactive feature selection. In 22nd International Conference on World Wide Web Companion (WWW), WWW'13 Companion, páginas 237-238, Rio de Janeiro, RJ, Brazil. http: / / dl . acm.org/ citation. cfm?id=2487788.2487910. Citado nas páginas 58 e 72 .

Marcacini, R. M., Hruschka, E. R., e Rezende, S. O. (2012c). On the use of consensus clustering for incremental learning of topic hierarchies. In 21st Brazilian Symposium on Artificial Intelligence (SBIA/BRACIS), Advances in Artificial Intelligence, páginas 112-121, Curitiba, PR, Brazil. Springer Berlin Heidelberg. http://dx.doi.org/10.1007/978-3-642-34459-6_12. Citado nas páginas 9, 60, 72, e 133.

Marcacini, R. M. e Rezende, S. O. (2010). Torch: a tool for building topic hierarchies from growing text collection. In WFA'2010: IX Workshop on Tools and Applications (Webmedia), páginas 1-3. Citado na página 119.

Marcacini, R. M. e Rezende, S. O. (2013). Incremental hierarchical text clustering with privileged information. In 13th ACM Symposium on Document Engineering (DocEng), páginas 231-232, Florence, Italy. ACM. http: //doi.acm.org/10.1145/2494266.2494296. Citado nas páginas 9, 45, 58,72 , e 133.

Milligan, G. e Cooper, M. (1985). An examination of procedures for determining the number of clusters in a data set. Psychometrika, 50(2):159-179. http: //dx.doi.org/10.1007/BF02294245. Citado nas páginas 95 e 98.

Milligan, G. e Cooper, M. (1986). A study of the comparability of external criteria for hierarchical cluster analysis. Multivariate Behavioral Research, 21(4):441-458. http://dx.doi.org/10.1007/BF02294245. Citado nas páginas 96 e 99.

Milligan, G. W. e Schilling, D. A. (1985). Asymptotic and finite sample characteristics of four external criterion measures. Multivariate Behavioral Research, 20(1):97-109. http://dx.doi.org/10.1207/s15327906mbr2001_6. Citado na página 99.

Miner, G., Elder, J., Hill, T., Nisbet, R., Delen, D., e Fast, A. (2012). Practical Text Mining and Statistical Analysis for Non-structured Text Data Applications. Academic Press. http://dl.acm.org/citation.cfm?id=2222696. Citado na página 43.

Mirkin, B. (2011). Choosing the number of clusters. Wiley Interdisciplinary Reviews: Data Mining and Knowledge Discovery, 1(3):252-260. http: / / dx . doi.org/10.1002/widm.15. Citado na página 97. 
Mirkin, B. (2012). Validation and Interpretation. Chapman \& Hall/CRC, Computer Science \& Data Analysis. http: / / www . crcpress . com/product / isbn/9781439838419. Citado nas páginas 99 e 100.

Mirzaei, A., Rahmati, M., e Ahmadi, M. (2008). A new method for hierarchical clustering combination. Intelligent Data Analysis, 12(6):549-571. http://iospress.metapress.com/content/20mh4381g2872ut0. Citado na página 104.

Mitchell, T. M. (1997). Machine Learning. McGraw-Hill Science/Engineering/Math, first edition ${ }^{a}$ edição. Citado na página 13.

Mladenic, D. e Grobelnik, M. (1998). Word sequences as features in textlearning. In 17th Electrotechnical and Computer Science Conference (ERK98), páginas 145-148. http://citeseerx.ist.psu.edu/viewdoc/summary? doi=10.1.1.49.2772. Citado nas páginas 43 e 47.

Mohri, M., Rostamizadeh, A., e Talwalkar, A. (2012). Foundations of Machine Learning. The MIT Press. http://mitpress.mit.edu/books/ foundations-machine-learning-0. Citado nas páginas 14, 95, e 100.

Nadeau, D. e Sekine, S. (2007). A survey of named entity recognition and classification. Lingvisticae Investigationes, 30(1):3-26. http://dx.doi.org/ doi:10.1075/li.30.1.03nad. Citado na página 124.

Nourashrafeddin, S., Milios, E., e Arnold, D. (2013). Interactive text document clustering using feature labeling. In DocEng'13: ACM symposium on Document engineering, páginas 61-70, New York, NY, USA. ACM. http://dx.doi.org/10.1145/2494266.2494279. Citado nas páginas 53, 54 , e 63.

Panniello, U. e Gorgoglione, M. (2012). Incorporating context into recommender systems: An empirical comparison of context-based approaches. 12(1):1-30. http://dx.doi.org/10.1007/s10660-012-9087-7. Citado na página 126.

Parameswaran, S. e Weinberger, K. Q. (2010). Large margin multi-task metric learning. 23:1867-1875. http://books.nips.cc/papers/files/nips23/ NIPS2010_0510.pdf. Citado na página 73.

Pechyony, D. e Vapnik, V. (2010). On the theory of learnining with privileged information. In Lafferty, J. D., Williams, C. K. I., Shawe-Taylor, J., Zemel, R. S., e Culotta, A., editors, Advances in Neural Information Processing Systems (NIPS), páginas 1894-1902. http://papers.nips.cc/paper/ 3960-on-the-theory-of-learnining-with-privileged-information. pdf. Citado nas páginas 4, 36, 41, e 74 .

Pechyony, D. e Vapnik, V. (2011). Fast optimization algorithms for solving svm+. In Statistical Learning and Data Science, páginas 3-24. (Chapman \& Hall-CRC Computer Science \& Data Analysis. Citado nas páginas 36 e 41. 
Pelleg, D. e Baras, D. (2007). K-means with large and noisy constraint sets. In 18th European Conference on Machine Learning, páginas 674682, Berlin, Heidelberg. Springer-Verlag. http://dx.doi.org/10.1007/ 978-3-540-74958-5_67. Citado nas páginas 25 e 26.

Podani, J. (2000). Simulation of random dendrograms and comparison tests: Some comments. Journal of Classification, 17(1):123-142. http://dx. doi. org/10.1007/s003570000007. Citado na página 104.

Qi, Z., Tian, Y., e Shi, Y. (2014). A new classification model using privileged information and its application. Neurocomputing, 129(0):146 - 152. http: //dx.doi.org/10.1016/j.neucom.2013.09.045. Citado nas páginas 40 e 41 .

Raghavan, H., Madani, O., e Jones, R. (2005). InterActive feature selection. In 19th International Joint Conference on Artificial Intelligence (IJCAI), páginas 841-846. http://ijcai.org/papers/1401.pdf. Citado na página 53.

Rand, W. (1971). Objective criteria for the evaluation of clustering methods. Journal of the American Statistical association, 66(336):846-850. Citado na página 99.

Ribeiro, B., Silva, C., Chen, N., Vieira, A., e das Neves, J. C. (2012). Enhanced default risk models with svm+. Expert Systems with Applications, 39:1014010152. http://dx.doi.org/10.1016/j.eswa.2012.02.142. Citado nas páginas 36,37 , e 41 .

Ribeiro, B., Silva, C., Vieira, A., Gaspar-Cunha, A., e das Neves, J. (2010). Financial distress model prediction using svm+. In International Joint Conference on Neural Networks (IJCNN), páginas 1-7. http://dx.doi .org/10 . 1109 / I JCNN.2010.5596729. Citado nas páginas 36 e 41.

Ricci, F., Rokach, L., e Shapira, B. (2011). Introduction to recommender systems. In Ricci, F., Rokach, L., Shapira, B., e Kantor, P. B., editors, Recommender Systems Handbook, páginas 1-35. Springer US. http: //dx.doi.org/10.1007/978-0-387-85820-3_1. Citado na página 123.

Rokach, L. (2010). A survey of clustering algorithms. In Data Mining and Knowledge Discovery, páginas 269-298. Springer, 2nd ${ }^{a}$ edição. http:// dx.doi.org/10.1007/978-0-387-09823-4_14. Citado nas páginas 1, 15, e 16 .

Rossi, R., Marcacini, R. M., e Rezende, S. O. (2013a). Benchmarking text collections for classification and clustering tasks. Relatório Técnico 395, Institute of Mathematics and Computer Sciences - University of São Paulo. http://www.icmc.usp.br/CMS/Arquivos/arquivos_enviados/ BIBLIOTECA_113_RT_395.pdf. Citado na página 68. 
Rossi, R. G., Marcacini, R. M., e Rezende, S. O. (2013b). Analysis of statistical keyword extraction methods for incremental clustering. In X Encontro Nacional de Inteligência Artificial e Computacional (ENIAC), páginas 1-12, Fortaleza, CE, Brazil. http://www.lbd.dcc.ufmg.br/colecoes/eniac/2013/ $0051 . p d f$. Citado nas páginas 10 e 72.

Rossi, R. G., Marcacini, R. M., e Rezende, S. O. (2014). Analysis of statistical keyword extraction methods for incremental clustering. Learning \& Nonlinear Models (L\&NLM). In review. Citado na página 10.

Rossi, R. G. e Rezende, S. O. (2011). Building a topic hierarchy using the bag-of-related-words representation. In Proceedings of the 11th ACM Symposium on Document Engineering, DocEng '11, páginas 195-204, New York, NY, USA. ACM. http://doi.acm.org/10.1145/2034691.2034733. Citado na página 52 .

Rousseeuw, P. J. (1987). Silhouettes: A graphical aid to the interpretation and validation of cluster analysis. Journal of Computational and Applied Mathematics, 20(0):53 - 65. http://dx.doi.org/10.1016/0377-0427(87) 90125-7. Citado na página 98.

Ruping, S. e Scheffer, T. (2005). Learning With Multiple Views. In ICML 2005 Workshop on Learning With Multiple Views. Citado na página 31.

Sahoo, N., Callan, J., Krishnan, R., Duncan, G., e Padman, R. (2006). Incremental hierarchical clustering of text documents. In 15th ACM International Conference on Information and Knowledge Management, CIKM '06, páginas 357-366, New York, NY, USA. ACM. http://doi.acm.org/10.1145/ 1183614.1183667 . Citado na página 3.

Salton, G., Allan, J., e Singhal, A. (1996). Automatic text decomposition and structuring. Information Processing \& Management, 32(2):127-138. Citado nas páginas 2,43 , e 46.

Salton, G. e Buckley, C. (1988). Term-weighting approaches in automatic text retrieval. An International Journal of Information Processing and Management, 24(5):513-523. Citado nas páginas 2 e 46.

Serra-Toro, C., Traver, V. J., e Pla, F. (2014). Exploring some practical issues of svm+: Is really privileged information that helps? Pattern Recognition Letters, 42(0):40 - 46. http://dx. doi.org/10.1016/j.patrec.2014.01. 013. Citado nas páginas 39 e 41 .

Sharmanska, V., Quadrianto, N., e Lampert, C. H. (2013). Learning to rank using privileged information. In IEEE International Conference on Computer Vision (ICCV), páginas 825-832. http://dx.doi.org/10.1109/ICCV. 2013.107. Citado nas páginas 39 e 41.

Sinoara, R. A., Sundermann, C. V., Marcacini, R. M., Domingues, M. A., e Rezende, S. O. (2014). Named entities as privileged information for hierarchical 
text clustering. In 18th International Database Engineering \& Applications Symposium (IDEAS), páginas 1-12, Porto, Portugal. No prelo. Citado nas páginas 10, 124, e 128.

Sneath, P. (1957). The application of computers to taxonomy. Journal of General Microbiology, 17(1):201-226. Citado na página 20.

Sokal, R. R. e Michener, C. D. (1958). A statistical method for evaluating systematic relationships. University of Kansas Scientific Bulletin, 28:14091438. Citado na página 21.

Sorensen, T. (1948). A method of establishing groups of equal amplitude in plant sociology based on similarity of species content and its application to analyses of the vegetation on Danish commons. Biologiske Skrifter, 5(5):3543. Citado na página 21.

Steinbach, M., Karypis, G., e Kumar, V. (2000). A comparison of document clustering techniques. In KDD'2000: Workshop on Text Mining, páginas 120. Citado na página 21.

Strehl, A. e Ghosh, J. (2003). Cluster ensembles - a knowledge reuse framework for combining multiple partitions. Journal of Machine Learning Research, 3:583-617. http://dx.doi.org/10.1162/153244303321897735. Citado nas páginas 23 e 24 .

Sundermann, C. V., Domingues, M. A., Marcacini, R. M., e Rezende, S. O. (2014). Using topic hierarchies with privileged information to improve context-aware recommender systems. In Brazilian Conference on Intelligent System (BRACIS), páginas 1-6, São Carlos, SP, Brazil. No prelo. Citado nas páginas 10 e 129.

Tan, C.-M., Wang, Y.-F., e Lee, C.-D. (2002). The use of bigrams to enhance text categorization. Information Processing and Management, 38(4):529-546. http://dx.doi.org/10.1016/S0306-4573(01)00045-0. Citado nas páginas 43 e 47 .

Tan, P.-N., Steinbach, M., e Kumar, V. (2005). Introduction to Data Mining. Addison-Wesley Longman Publishing Co., Inc., Boston, MA, USA. http: //www-users.cs.umn.edu/ kumar/dmbook/. Citado nas páginas 14, 16, $22,29,95,96$, e 100.

Tibshirani, R. e Walther, G. (2005). Cluster validation by prediction strength. Journal of Computational and Graphical Statistics, 14(3):511-528. http: //dx.doi.org/10.1198/106186005x59243. Citado na página 100.

Vaithyanathan, S. e Dom, B. (1999). Model selection in unsupervised learning with applications to document clustering. In International Conference on Machine Learning (ICML), páginas 433-443, San Francisco, CA, USA. Morgan Kaufmann Publishers Inc. http://dl.acm.org/citation.cfm?id= 657778. Citado nas páginas 3 e 95. 
Vapnik, V. e Vashist, A. (2009). A new learning paradigm: Learning using privileged information. Neural Networks, 22(5-6):544 - 557. http://dx. doi.org/10.1016/j.neunet.2009.06.042. Citado nas páginas 4, 5, 33, $34,35,39,41,44,45,74,75,77,78,81,84,87,93$, e 132.

Vendramin, L., Campello, R. J. G. B., e Hruschka, E. R. (2010). Relative clustering validity criteria: A comparative overview. Statistical Analysis and Data Mining, 3(4):209-235. http://dx.doi.org/10.1002/sam.10080. Citado nas páginas $3,95,96,97,98$, e 99 .

Volkovich, Z., Barzily, Z., e Morozensky, L. (2008). A statistical model of cluster stability. Pattern Recognition, 41(7):2174-2188. http://dx.doi.org/10. $1016 / j$.patcog.2008.01.008. Citado na página 100.

Wagstaff, K., Cardie, C., Rogers, S., e Schrödl, S. (2001). Constrained k-means clustering with background knowledge. In Proceedings of the Eighteenth International Conference on Machine Learning, páginas 577-584, San Francisco, CA, USA. Morgan Kaufmann Publishers Inc. http://dl.acm.org/ citation. cfm?id=655669. Citado na página 25.

Wang, D. e Li, T. (2010). Document update summarization using incremental hierarchical clustering. In Proceedings of the 19th ACM International Conference on Information and Knowledge Management, páginas 279-288, New York, NY, USA. ACM. http://doi.acm.org/10.1145/1871437.1871476. Citado na página 29.

Wang, J., Wu, S., Vu, H. Q., e Li, G. (2010). Text document clustering with metric learning. In International ACM Conference on Research and Development in Information Retrieval (SIGIR), páginas 783-784. http://doi . acm . org/10.1145/1835449.1835614. Citado na página 73.

Weinberger, K. Q. e Saul, L. K. (2009). Distance metric learning for large margin nearest neighbor classification. Journal of Machine Learning Research, 10:207-244. http://www.jmlr.org/papers/volume10/weinberger09a/ weinberger09a.pdf. Citado nas páginas 73 e 80.

Wu, J., Liu, H., Xiong, H., e Cao, J. (2013). A theoretic framework of k-meansbased consensus clustering. In Twenty-Third International Joint Conference on Artificial Intelligence, páginas 1799-1805. AAAI Press. http://dl . acm. org/citation. cfm?id=2540128.2540386. Citado na página 24.

Xiang, S., Nie, F., e Zhang, C. (2008). Learning a mahalanobis distance metric for data clustering and classification. Pattern Recognition, 41(12):36003612. http://dx.doi.org/10.1016/j.patcog.2008.05.018. Citado na página 3.

Xing, E. P., Jordan, M. I., Russell, S., e Ng, A. (2002). Distance metric learning with application to clustering with side-information. In Advances in Neural Information Processing Systems (NIPS), páginas 505-512. http://books . 
nips.cc/papers/files/nips15/AA03.pdf. Citado nas páginas 2, 73, 78, 79,84 , e 85.

Xu, R. e Wunsch, D. (2008). Clustering. Wiley-IEEE Press, IEEE Press Series on Computational Intelligence. http://dl.acm.org/citation.cfm? id=1483087. Citado nas páginas xi, 1, 14, 19, 22, 28, 29, 95, 96, 97, e 99.

Yang, H. e Patras, I. (2013). Privileged information-based conditional regression forest for facial feature detection. In 10th IEEE International Conference and Workshops on Automatic Face and Gesture Recognition (FG), páginas 16. http://dx.doi.org/10.1109/FG.2013.6553766. Citado nas páginas 38 e 41 .

Yang, L. (2007). An overview of distance metric learning. Technical report, School of Computer Science, Carnegie Mellon University. http://cs.cmu . edu/ liuy/dist_overview.pdf. Citado na página 73.

Yang, L., Jin, R., Sukthankar, R., e Liu, Y. (2006). An efficient algorithm for local distance metric learning. In AAAI Conference on Artificial Intelligence, volume 2. Www.aaai.org/Papers/AAAI/2006/AAAI06-087.pdf. Citado nas páginas 73 e 80 .

Yang, Z., Zhang, L., Yan, J., e Li, Z. (2003). Using association features to enhance the performance of naïve bayes text classifier. In International Conference on Computational Intelligence and Multimedia Applications, página 336. IEEE Computer Society. http://dx.doi .org/10.1109/ICCIMA. 2003.1238148 . Citado na página 43.

Zeng, H. e Cheung, Y.-M. (May). Semi-supervised maximum margin clustering with pairwise constraints. IEEE Transactions on Knowledge and Data Engineering, 24(5):926-939. Citado na página 25.

Zhang, D., Wang, F., Zhang, C., e Li, T. (2008). Multi-view local learning. In AAAI'08: International Conference on Artificial Intelligence, páginas 752-757. AAAI Press. Citado na página 31.

Zhang, T., Ramakrishnan, R., e Livny, M. (1996). Birch: an efficient data clustering method for very large databases. ACM SIGMOD Record, 25(2):103114. Citado nas páginas 28 e 29.

Zhang, W., Yoshida, T., Tang, X., e Wang, Q. (2010). Text clustering using frequent itemsets. Knowledge-Based Systems, 23(5):379 - 388. http:// dx.doi.org/10.1016/j.knosys.2010.01.011. Citado na página 51.

Zhao, Y. e Karypis, G. (2002). Evaluation of hierarchical clustering algorithms for document datasets. In CIKM '02: Proceedings of the 11th international conference on Information and Knowledge Management, páginas 515-524, New York, NY, USA. ACM. Citado nas páginas 22 e 29. 
Zhao, Y., Karypis, G., e Fayyad, U. (2005). Hierarchical clustering algorithms for document datasets. Data Mining and Knowledge Discovery, 10(2):141168. http://dx.doi.org/10.1007/s10618-005-0361-3. Citado nas páginas $1,16,21,22$, e 29 .

Zheng, L., Li, T., e Ding, C. (2010). Hierarchical ensemble clustering. In International Conference on Data Mining (ICDM), páginas 1199-1204. http : //dx.doi.org/10.1109/ICDM.2010.98. Citado na página 104.

Zhou, D. e Burges, C. J. C. (2007). Spectral clustering and transductive learning with multiple views. In ICML'07: International Conference on Machine learning, páginas 1159-1166, New York, NY, USA. ACM. Citado na página 31.

Zhuang, F., Karypis, G., Ning, X., He, Q., e Shi, Z. (2012). Multi-view learning via probabilistic latent semantic analysis. Information Sciences, 199(0):20 30. Citado na página 31 . 